\title{
Low-Drift Micro \\ Flow Sensors
}

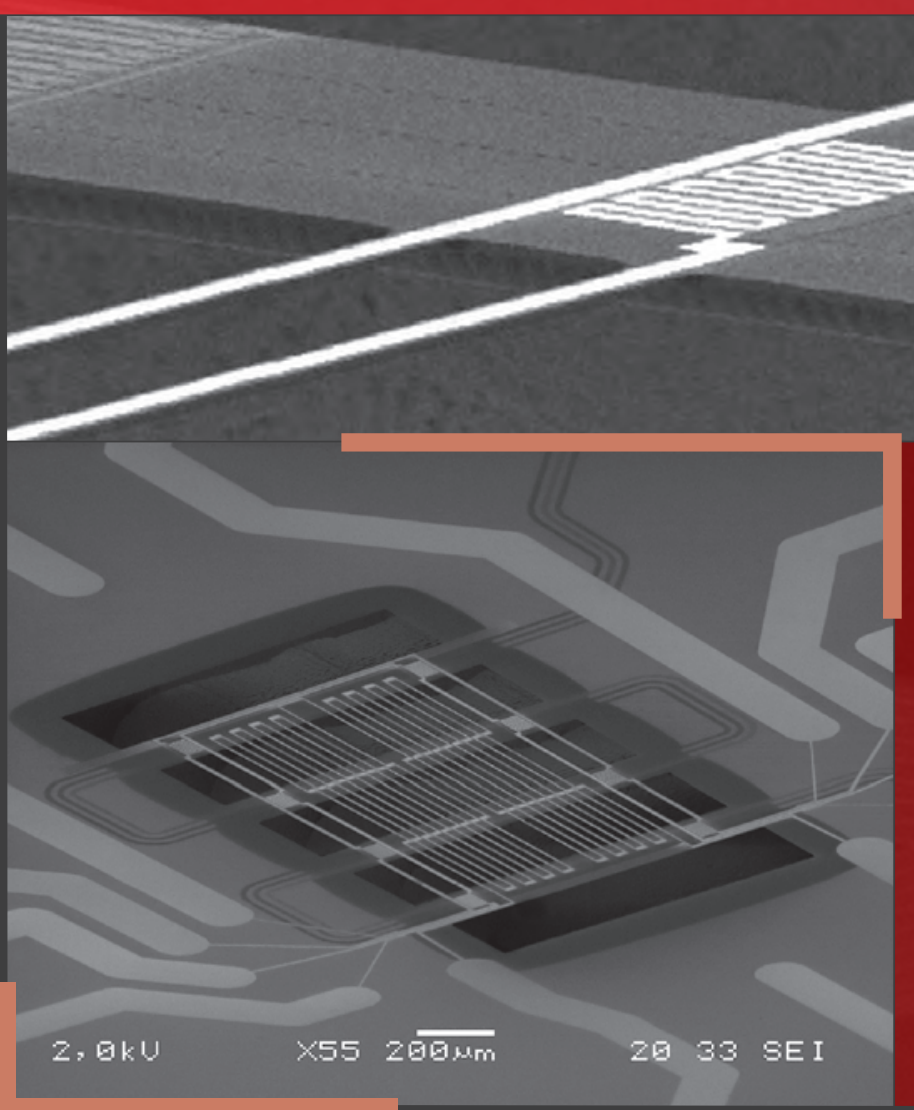

MARCEL DiJKSTRA 

LOW-DRIT MCRO FLOW SENSORS



MARCEL DIJKSTRA 
The research described in this thesis was carried out at the Transducers Science \& Technology group at the MESA ${ }^{+}$Institute for Nanotechnology at the University of Twente, Enschede, The Netherlands. The project was financially supported by the Dutch Technology Foundation (STW).

Promotiecommissie:

Voorzitter

Prof. dr. ir. A.J. Mouthaan Universiteit Twente

\section{Secretaris}

Prof. dr. ir. A.J. Mouthaan Universiteit Twente

\section{Promotor}

Prof. dr. M.C. Elwenspoek Universiteit Twente Assistent-Promotor

Dr. ir. R.J. Wiegerink Universiteit Twente

Leden

Prof. dr. ir. A. van den Berg Universiteit Twente

Prof. dr. J.G.E. Gardeniers Universiteit Twente

Prof. dr. ir. P.P.L. Regtien Universitiet Twente

Prof. dr. J. Schmitz

Universitiet Twente

Prof. dr. G. Stemme

Royal Institute of Technolgy, Stockholm

Low-drift micro flow sensors

Dijkstra, Marcel

Ph.D. thesis, University of Twente, Enschede, The Netherlands

ISBN: 978-90-365-2856-6 DOI:10.3990/1.9789036528566

\section{Cover:}

Micrographs of Ambient Temperature-Gradient Compensated Flow Sensors (Chap. 7).

Power Spectral Density of Low-Drift Flow Sensor Response (Fig. 5.11).

Printed by Gildeprint, Enschede, The Netherlands

Copyright (C) 2009 M. Dijkstra, Enschede, The Netherlands

All rights reserved. No part of this publication may be reproduced or stored in a retrieval system without the prior permission of the author. 


\title{
LOW-DRIFT MCRO FLOW SENSORS
}

\author{
PROEFSCHRIFT
}

ter verkrijging van

de graad van doctor aan de Universiteit Twente,

op gezag van de rector magnificus

prof. dr. H. Brinksma,

volgens besluit van het College voor Promoties

in het openbaar te verdedigen

op vrijdag 12 juni 2009 om 13.15 uur

door

Marcel Dijkstra

geboren op 28 april 1978

te Groningen 
Dit proefschrift is goedgekeurd door de promotor en de assistent-promotor: Prof. dr. M.C. Elwenspoek

Dr. ir. R.J. Wiegerink 




\section{Contents}

1 Introduction 1

1.1 General Introduction 1

1.2 Micro Flow Sensors 1

1.3 Micro Thermal Flow Sensors 4

1.3.1 External Forced-Convection Sensors 4

1.3.2 Internal Forced-Convection Sensors 6

1.4 Aim of the Research 9

1.5 Outline of the Thesis 9

References $\quad 11$

2 Low-Drift Flow Sensing 15

2.1 Introduction $\quad 15$

2.2 Thin-Film Transducers 15

2.2.1 Thin-Film Resistors 15

$\begin{array}{ll}\text { 2.2.2 Thin-Film Thermopiles } & 18\end{array}$

2.3 Flow Sensing Concepts 21

2.2.1 Constant-Power Calorimetric Sensing with Resistors 21

2.2.2 Constant-Power Calorimetric Sensing with Thermopiles 23

$\begin{array}{lll}\text { 2.2.3 Temperature-Balancing } & 24\end{array}$

Calorimetric Sensing with Thermopiles

$\begin{array}{ll}\text { References } & 26\end{array}$

3 Surface Channel Technology 29

3.1 Introduction 29

3.2 Surface Microchannel Concept 31

3.2.1 Surface Microchannel Fabrication 31

3.2.2 Transducer and Surface Micromachining Integration 34

3.2.3 Surface Microchannel Release 34

3.2.4 Fluidic Entrance Holes to the Surface Microchannels 36 
ii. Contents

3.3 Channel Profile Etching 39

3.4 Surface Microchannel Process Results 40

3.4.1 Release of Device Templates by KOH Etching 40

3.4.2 Release of Device Templates by $\mathrm{SF}_{6}$ Plasma 43

3.4.3 Packaging of Surface Microchannel Devices 44

3.5 Conclusions 46

References 46

4 Miniaturised Calorimetric Flow Sensor $\quad 49$

4.1 Introduction $\quad 49$

4.2 Sensor Design 50

4.3 Sensor Fabrication 51

4.4 Micro Thermal Flow Sensors 54

4.4.1 Fluidic-Thermal CFDRC Model 54

4.4.2 Thermal FVM Model 58

4.5 Experimental Setup $\quad 60$

4.6 Experimental and Model Results $\quad 60$

4.7 Conclusions 63

$\begin{array}{ll}\text { References } & 63\end{array}$

5 Low-Drift Flow Sensor with Thermopile-Based Power Feedback 65

5.1 Introduction $\quad 65$

5.2 Sensor Fabrication 66

5.3 Sensor Modelling

$\begin{array}{ll}5.4 & \text { Experimental Results }\end{array}$

\begin{tabular}{ll}
5.5 & Conclusions \\
\hline
\end{tabular}

$\begin{array}{ll}\text { References } & 78\end{array}$

6 Low-Drift U-Shaped Thermopile Flow Sensor 79

6.1 Introduction $\quad 79$

6.2 Sensor Fabrication $\quad 82$ 
6.3 Experimental Results $\quad 84$

$\begin{array}{lll}\text { 6.4. Conclusions } & 87\end{array}$

$\begin{array}{ll}\text { References } & 87\end{array}$

Ambient Temperature-Gradient

7 Compensated Low-Drift Thermopile Flow Sensor 89

7.1 Introduction 89

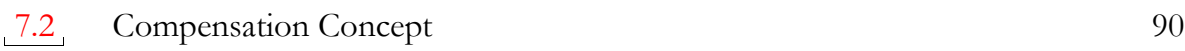

7.3 Sensor Fabrication $\quad 90$

7.4 Sensor Modelling 93

7.5 Experimental Results $\quad 94$

7.5.1 Flow-Rate Sensitivity 94

7.5.2 Ambient Temperature-Gradient Sensitivity 95

$\begin{array}{ll}\text { 7.6 Conclusions } & 97\end{array}$

$\begin{array}{lr}\text { References } & 98\end{array}$

AC-Driven

8 Temperature-Balancing Flow Sensor with Power Feedback 99

8.1 Introduction 99

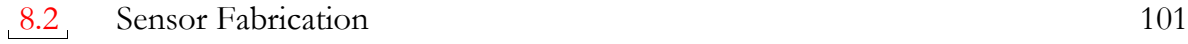

8.3 Sensor Modelling 103

8.4 Experimental Results 105

8.5 Conclusions 111

References 112

Nano-Nozzle Electrospray Emitters

9 Fabricated by a Micro- to Nano-Fluidic Via Technology 113

9.1 Introduction 113

9.2 Experimental 114

9.2.1 Micro- to Nano-Fluidic Vias 114

9.2.2 Filling Experiments 115

9.2.3 Nano-Nozzle Electrospray Emitters 118

$\begin{array}{ll}\text { 9.2.4 Electrospray-Ionisation Setup } & 118\end{array}$ 
iv Contents

9.3 Results and Discussion 120

9.4 Conclusions 121

References 122

10 Conclusions and Future Research 125

10.1 Surface Channel Technology 125

10.2 Material-Drift Compensation 126

10.3 Temperature-Gradient Compensation 127

10.4 Outlook on Low-Drift Micro Flow Sensing 127

$\begin{array}{ll}\text { References } & 129\end{array}$

Appendix

A Process Documentation 131

A.1 Surface Microchannel Fabrication 131

A.2 Al/poly-Si ${ }^{++}$Thermopile Fabrication 134

A.3 Surface Microchannel SF 6 Plasma Release 137

\begin{tabular}{lr} 
Summary & 139 \\
\hline
\end{tabular}

$\begin{array}{ll}\text { Samenvatting } & 140\end{array}$

\begin{tabular}{lr} 
List of Publications & 142 \\
\hline
\end{tabular}

\begin{tabular}{ll} 
Dankwoord & 144 \\
\hline
\end{tabular}

$\begin{array}{ll}\text { Biography/ Biografie } & 146\end{array}$ 


\section{Introduction}

The research described in this thesis concerns the development of low-drift micro flow sensors for the accurate measurement of minute amounts of liquid flow. In this chapter an introduction is given on micro flow sensors, with emphasis on thermal flow sensors applicable to microfluidic systems. The main aim of this work is to increase the accuracy of microfluidic thermal flow sensors. The chapter ends with an outline of the scientific work presented, which has lead to the realisation of low-drift micro thermal flow sensors, with compensation for external temperature gradients.

\subsection{General Introduction}

Flow sensors find applications in many areas in industry. Applications range from motorcars, process industry, analysis and synthesis in chemistry, pharmacy, biology and medicine. The emerging fields of micro total-analysis systems (micro-TAS), micro reactors and bio-MEMS drives the need for further miniaturisation of flow sensors capable of measuring minute amounts of liquid flow. Miniaturisation has intrinsic advantages such as high speed, small amounts of fluid required for analysis and portability, but perhaps more importantly fluidic components are being integrated in whole systems. The control of these systems is only possible with sensors measuring quantities such as pressure, temperature and flow. The need for small and reliable sensors makes flow sensing an important application in micro systems technology.

Micro high-pressure liquid chromatography (micro-HPLC) and other micro-TAS applications require liquid flow-rate resolution down to the $\mathrm{nl} \cdot \mathrm{min}^{-1}$ range. Advancements in nanofluidics demand an even further increase in flow-rate resolution. This can only be achieved with the development of highly-sensitive micro flow sensors. These sensors should make optimum use of the liquid flow, while applying a transduction principle with maximum signal-to-noise ratio. Resolution can be increased further by proper electronic amplification of the sensor signal, while reducing noise influences. Ultimately, external disturbances and drift of transducer properties have to be compensated for, or otherwise drift will render the flow sensor useless for accurate determination of flow rates in the nl. $\mathrm{min}^{-1}$ range and below.

\subsection{Micro Flow Sensors}

The development of instrumentation for the measurement of fluid flow has a long history dating back to ancient times. A whole range of flow sensing principles has since been studied by a great many scientists in not more than the last four hundred years [1.1]. Many of these principles are applied in the miniaturisation of flow sensors 
[1.2-1.4], but not all principles are equally suited for downscaling and some find difficulties in microfluidic system integration.

\section{Mechanical Flow Sensors}

One of the most direct ways in measuring fluid flow is by utilising the mechanical drag force imposed by a moving fluid on a structure. This principle has been adopted by nature, where by means of natural selection [1.5] highly-flow-sensitive mechoreseptive hairs have evolved in e.g. crickets, which are yet unrivalled by intelligent designs (Krijnen et al. [1.6-1.8]). Figure 1.1a shows biomimetic sensory hairs made from SU-8, where electrodes are integrated for the capacitive measurement of low frequency sound waves [1.6]. Other types of mechanical flow sensors include lift-force and

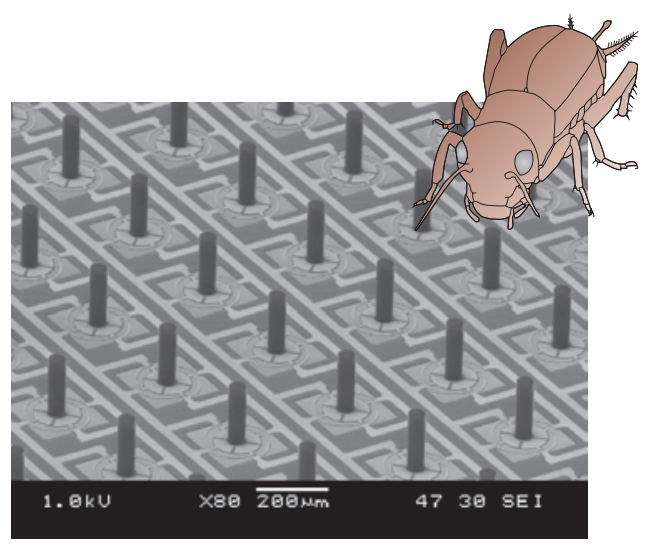

a)

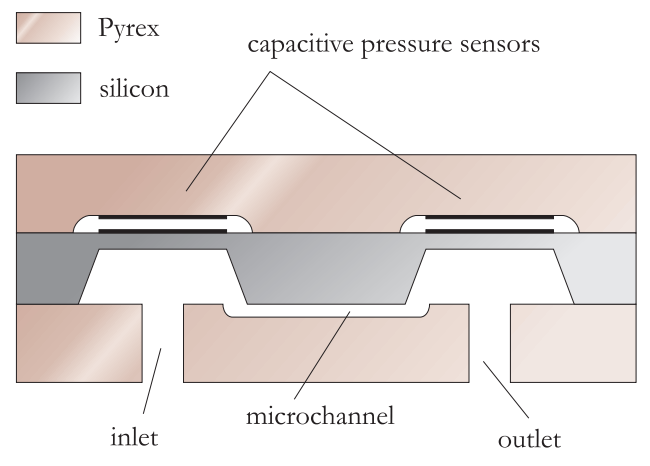

c)

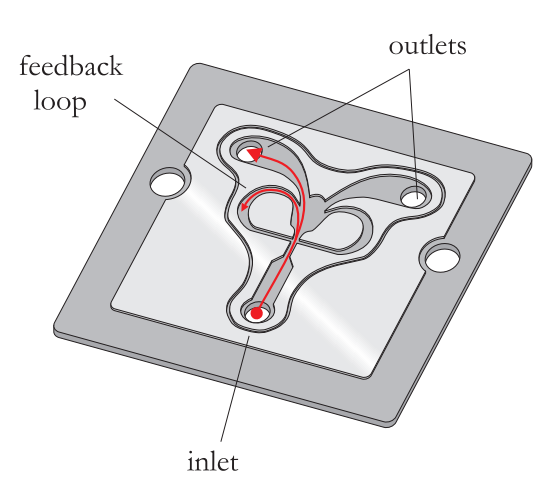

b)

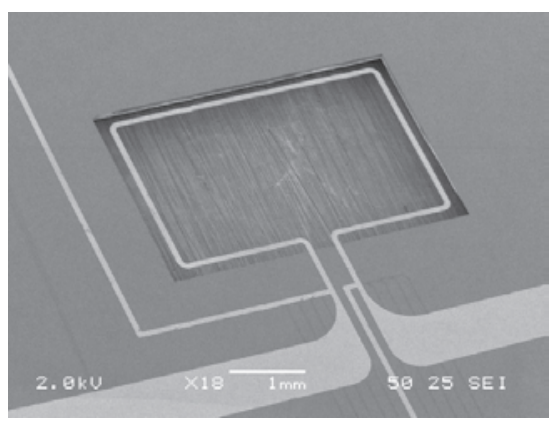

d)

Fig. 1.1 Micro flow sensors based on a) drag-force by Dijkstra et al. [1.6], b) the Coanda effect by Gebhard et al. [1.10], c) differential-pressure by Oosterbroek et al. [1.15] and d) the Coriolis effect by Haneveld et al. [1.20]. 
skin-friction sensors [1.2]. Recently, Attia et al. [1.9] determined liquid flow rate by measuring the extension of a spring structure contained in a microfluidic channel. Flow rates down to $\mathrm{nl} \cdot \mathrm{min}^{-1}$ could be obtained through optical microscope inspection. A fully functional flow sensor has not yet been demonstrated.

\section{Oscillatory Flow Sensors}

Extremely accurate flow sensing can be obtained using fluidic oscillators [1.10-1.13]. Figure 1.1b shows a Coanda flow meter fabricated in the LIGA process by Gebhard et al. [1.10], where an oscillating fluid jet is generated between two outlets. The fluid flows to one of the outlets, due to the Coanda effect. Switching occurs by a feedback loop, which counteracts the Coanda effect. The frequency of oscillation $\omega$ is a measure of the flow velocity $v$ and is governed [1.12] by the Strouhal number $S_{t}=\omega D_{b} / v$, being constant or linearly dependent on the Reynolds number $\mathrm{R} e=v D_{b} / v$, with $D_{b}$ the hydraulic diameter and $v$ the kinematic viscosity of the fluid. The oscillation frequency can be picked up by e.g. pressure or thermal transducers in the feedback loops. Coanda flow sensors are used as highly stable reference flow meters in the gas industry [1.13], because the oscillating digital output of the sensor allows transducer properties to drift over time, while retaining sensor accuracy. However, Coanda flow sensors are not suitable for miniaturisation as the Strouhal number becomes dependent on a decreasing Reynolds number and oscillations finally cease because of viscous damping [1.14].

\section{Differential-Pressure Flow Sensors}

Efficient integration with a microfluidic system can be obtained with differentialpressure flow sensors [1.2]. Oosterbroek et al. [1.15, 1.16] have demonstrated measurement of ethanol in the $\mu l \cdot \mathrm{s}^{-1}$ range by measuring the pressure drop over a microchannel using capacitive pressure sensors (Fig. 1.1c). The resolution of the sensor can possibly be improved, applying more sensitive pressure sensors and by increasing the hydraulic resistance of the microchannel, which constitutes in the largest bottleneck for the flow.

\section{Coriolis Flow Sensors}

Coriolis flow sensors make use of the Coriolis force $\vec{F}_{C}^{\prime}=2 \vec{\phi} \times \partial_{t} \vec{\theta}$, acting along a vibrating tube $\partial_{t} \theta$, guiding a mass flow rate $\phi$. Enokson et al. [1.17] were the first to develop a micromachined Coriolis flow sensor, followed by Sparks et al. [1.18]. The Coriolis effect is not directly suitable for downscaling. However, Mehendale [1.19] demonstrated that with an intelligent large-scale sensor design mass flow rates in the $\mathrm{g} \cdot \mathrm{h}^{-1}$ range $\left(3.3 \mathrm{ml} \cdot \mathrm{min}^{-1}\right.$ for water $)$ can be measured. 
Figure 1.1d shows the realisation of a Coriolis resonance tube by Haneveld et al. [1.20]. A fully-functional Coriolis flow sensor was created by Haneveld et al. [1.21], using integrated comb-like capacitive-readout electrodes and Lorentz-force actuation. Liquid flow rates up to $20 \mu \mathrm{l} \cdot \mathrm{min}^{-1}$ have been measured with $0.4 \mu \mathrm{l} \cdot \mathrm{min}^{-1}$ resolution.

\subsection{Micro Thermal Flow Sensors}

Most sensing principles described in Sec. 1.2 have gradually resulted in flow sensors with higher sensitivity. However, micro thermal flow sensors are among the most sensitive flow sensors for liquids to date. The most sensitive sensors are obtained with fully-heated freely-suspended microchannels. These micro thermal flow sensors can furthermore readily be integrated with other microfluidic components.

\subsubsection{External Forced-Convection Sensors}

Micromachined thermal flow sensors rely on the interaction of a fluid flow with heat produced by on-chip integrated heaters. In many cases the flow sensor chip is positioned directly in the flow or the chip is integrated in the wall of a conduit. Sensor characteristics depend in both cases on fluid boundary layers.

This is described by the Prandtl-Blasius boundary layer solution $[1.22,1.23]$ for a flat surface positioned parallel to the flow. The flow velocity $v$ stagnates on the surface, which results in a shear-stress boundary layer extending into the fluid. The boundary layer builds up in the direction of the flow ( $x$-direction), starting from the edge of the flat surface. The boundary layer thickness $\delta$, defined at the specific height above the flat plate where $v=0.99 v_{\infty}$ is given by (1.1), with $v_{\infty}$ the free-stream velocity and $R e_{x}=v_{\infty} x / v$ the Reynolds number depending on $x$. A thermal boundary layer (1.2) resides inside the fluidic boundary layer [1.22, 1.24], with the surface having an elevated temperature and with fluids $\alpha$ having a Prandtl number $\operatorname{Pr}=v / \rho c$ larger than one, with $\kappa$ the thermal conductivity, $\rho$ the density and $c$ the heat capacity of the fluid.

$$
\begin{gathered}
\delta=\frac{5 x}{\sqrt{\mathrm{R} e_{x}}} \\
\delta_{t h}=\delta \operatorname{Pr}^{-1 / 3}
\end{gathered}
$$

The heat flux normal to the surface can be related to the local temperature gradient also normal to the surface. This is expressed in the Nusselt number $N u_{x}=b_{x} x / \kappa$, with $h_{x}$ the heat transfer coefficient. The relation (1.3) is proportional to the thermal boundary layer thickness as this determines the local temperature gradient, where $\lambda$ is a constant specific to the sensor geometry $[1.24,1.25]$. The forced convection by fluid 
flow from an on-chip heater dissipating a fixed amount of power $P$ results in a temperature change $\Delta T$ of the heater. This anemometric sensor response is described by King's law (1.4), where $\alpha$ determines the contribution by conduction and $\beta$ determines the sensitivity to forced convection by the fluid flow.

$$
\begin{aligned}
N u_{x} & =\lambda \operatorname{Pr}^{1 / 3} \operatorname{Re}_{x}^{1 / 2} \\
\Delta T & =\frac{P}{\alpha+\beta R e^{1 / 2}}
\end{aligned}
$$

Thermal anemometers can e.g. be used to measure wind speed. Oudheusden et al. [1.26] measured temperature difference between thermopiles integrated at the edge of a square chip (Fig. 1.2a). The whole chip is elevated in temperature by dissipating heat in on-chip resistors. In this way, the wind direction and wind speed can be obtained from the thermal boundary layer [1.26-1.28]. A boundary layer is not present at low flow speed. Instead, a temperature disturbance linear with flow speed can be measured [1.22], which is the calorimetric regime of the flow sensor. The temperature difference can be measured with resistors [1.29, 1.30] or thermopiles [1.31-1.34].

Putten et al. [1.35] demonstrated that long-term drift in transducer properties can be eliminated by the alternating direction method, whereby the chip is rotated in the flow. This allows the distinction between sensor-drift and flow-velocity signals.

Brushi et al. [1.36, 1.37] used an operational amplifier to control the power between two heaters cancelling the voltage generated by two integrated thermopiles. The control power provides a measure for the flow speed, according to the temperature-

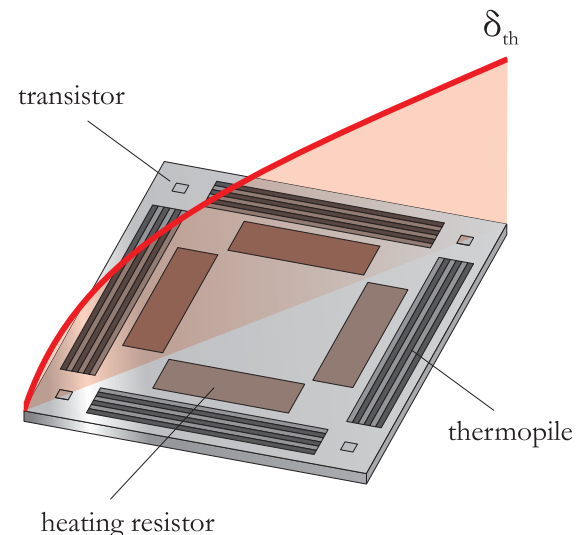

a)

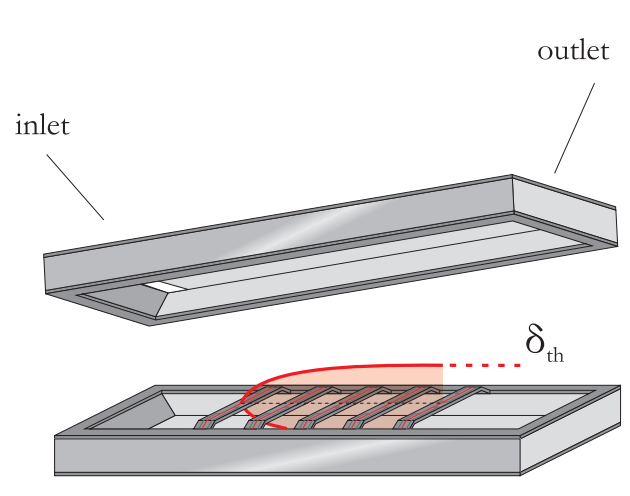

b)

Fig. 1.2 Thermal flow sensors where thermal boundary layers define flow sensor characteristics, with a) wind direction flow sensor by Oudheusden et al. [1.26] and b) microchannel distributed thermal flow sensor by van Baar et al. [1.41]. 
balancing anemometer principle [1.38].

Microfluidic system integration requires sensor chips with integrated microchannels, such that the flow rate through the microchannel can be determined accurately $[1.39,1.40]$. Van Baar et al. [1.41, 1.42] realised distributed sensing arrays on micro beams suspended in a microchannel created at the interface between two silicon chips (Fig. 1.2a). This means however that the fluid is in contact with the beams. The structures can be used for the determination of flow rate and other fluid parameters. The fluid boundary layer thickness $\delta$ is constant after the fluid becomes fully developed over the suspended beams. This means that the Nusselt number (1.3) is no longer dependent on position [1.43].

Meng et al. $[1.44,1.45]$ realised a similar sensing array, where heaters were integrated on top of a Parylene membrane suspended over a microchannel etched in a silicon substrate. Liquid flow rate down to $0.5 \mu \mathrm{l} \cdot \mathrm{min}^{-1}$ could be measured. In this type of microchannel flow sensors [1.39-1.45], the fluid never becomes fully heated, as it approaches the chip temperature close to the wall of the microchannel.

\subsubsection{Internal Forced-Convection Sensors}

Highest sensitivity can be obtained when the complete fluid is heated. This requires the integration of freely suspended microchannels, thermally isolated from the chip. With on-chip microchannel capillaries the fluid flow develops to a fully developed flow profile within the hydrodynamic entrance length $X_{b}(1.5)$, where $R e_{D b}=Q D_{b} / A v$ is the Reynolds number based on the average flow velocity $Q / A$ and $D_{b}$ the hydraulic diameter, which is equal to the capillary diameter $D$ in case of a circular perimeter [1.46].

$$
X_{b}=0.05 R e_{D_{b}} D_{b}
$$

A thermal entrance length $X_{t}=\operatorname{Pr} X_{b}$ is defined for a capillary with an already established fully developed flow profile, where the fluid is at a different temperature than the wall or in case a wall heat flux is defined. The developing thermal profile along the capillary is expressed by the non-dimensional Greatz number $G$ \%, where a thermal fully developed profile beyond $X_{t}$ corresponds with $G z<16$.

For a cylindrical capillary with a $1 \mu \mathrm{l} \cdot \mathrm{min}^{-1}$ water flow rate $Q$ the Reynolds number is $R_{e_{D}}=20 \mu \mathrm{m} / D$ and the hydrodynamic entrance length $X_{b}$ is $1 \mu \mathrm{m}$. The thermal entrance length $X_{t}$ for a uniformly heated capillary is $7 \mu \mathrm{m}$ independent of the tube diameter $D$, but directly proportional to the flow rate $Q$. The thermal entrance length for micromachined flow sensors in the $\mathrm{nl} \cdot \mathrm{min}^{-1}$ range is therefore sufficiently short to be considered thermally fully developed over the complete length of the microchannel used for flow metering. 
The forced convection in a thermally fully developed microchannel is determined by the total flow rate $Q$ in the microchannel. The Nusselt number is constant, with an order of magnitude close to one for a cylindrical channel [1.46]. The radial temperature gradient is therefore independent of the Reynolds number, but directly proportional to the wall heat-flux. The temperature profile along the microchannel can be understood by determining the heat conduction through the whole sensor structure and surrounding air, taking into account the radial heat flux from the microchannel due to forced convection by a given flow rate $\mathcal{Q}$ [1.47]. The radial heat flux due to convection is again dependent on the gradient in the temperature profile along the microchannel.

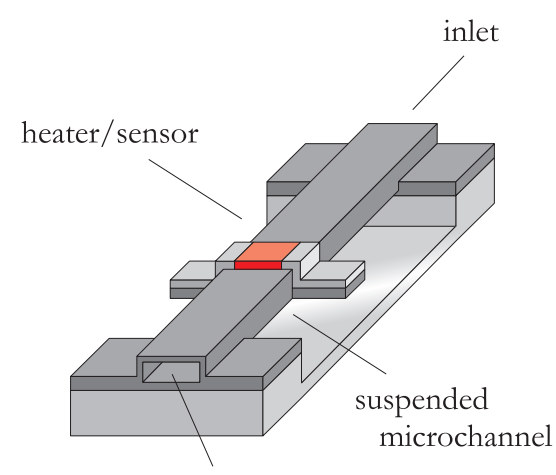

outlet

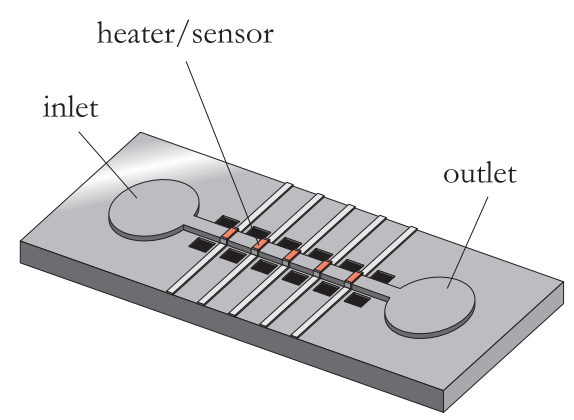

b)

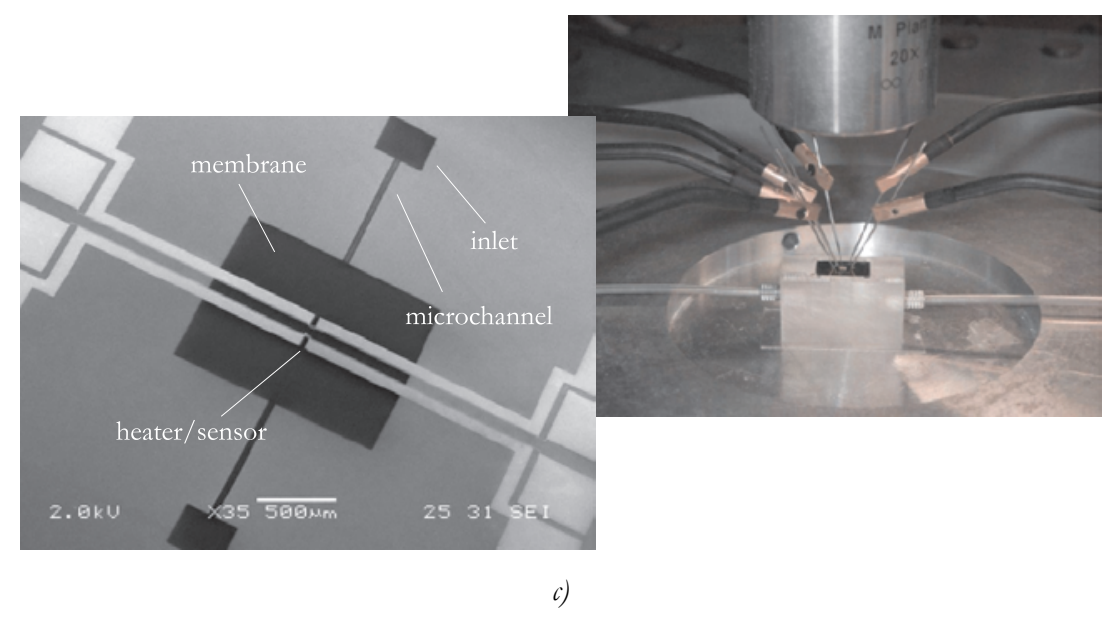

Fig. 1.3 Thermal flow sensors where thermally-fully-developed suspended microchannels are used to measure liquid flow, with a) nano-fluidic flow sensor by $W u$ et al. $[1.48]$, b) nano flow sensor with carbon sensing elements by Mizuno et al. [1.49] and c) realised $\omega-2 \omega$ nano flow sensor [1.50]. 
Wu et al. [1.48] were the first to use a suspended $2 \mu \mathrm{m}$ high microchannel over an etched cavity (Fig. 1.3a) for thermal flow sensing. Flow rate resolution down to $4 \mathrm{nl} \cdot \mathrm{min}^{-1}$ for water was obtained with anemometry, using an integrated poly-Si heater/sensor. Mizuno et al. [1.49] used high-temperature sensitive carbon doped Parylene resistors (Fig. 1.3b) resulting in a higher flow rate resolution. Figure 1.3c shows a realised micro flow sensor [1.50], where a microchannel is suspended across a membrane. Heat waves with a $\omega-2 \omega$ lock-in sensing method were used to measure water flow rate down to a few $\mathrm{nl} \cdot \mathrm{min}^{-1}$.

Freely-suspended-microchannel flow sensors [1.48-1.53] have been fabricated using sacrificial layer etching, where the height of the microchannel is limited by technology. The hydraulic resistance $R=\Delta p / Q$ of a rectangular microchannel $R_{\text {rect }}[1.16]$ is significantly being determined by the microchannel height $h$, where $w$ is the width and $l$ the length of the microchannel and $\mu$ the dynamic viscosity.

$$
\mathrm{R}_{\text {rect }}=\frac{12 \mu l}{b^{3} w}
$$

In the $\omega$ - $2 \omega$ sensor design, the hydraulic resistance $R_{\text {rect }}$ is $29 \mathrm{bar} / \mu \mathrm{l} \cdot \mathrm{min}^{-1}$ for water flow through the microchannel (Fig. 1.3c), where the microchannel height is $1 \mu \mathrm{m}$, the width is $100 \mu \mathrm{m}$ and the length is $1.3 \mathrm{~mm}$. This large hydraulic resistance is impractical for micro flow sensing applications.

Commercially available micro thermal flow sensors [1.54-1.57] (Fig. 1.4) in the $\mathrm{nl} \cdot \mathrm{min}^{-1}$ flow range make use of cylindrical capillaries made either of stainless steel, PEEK ${ }^{\mathrm{TM}}$ or fused silica. The hydraulic resistance $R_{\text {circ }}(1.7)$ of the cylindrical capillary

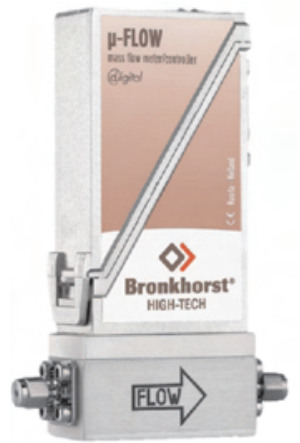

a)

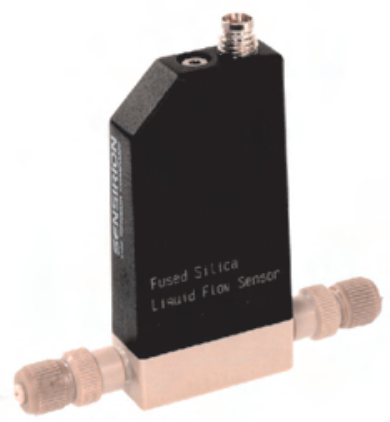

b)

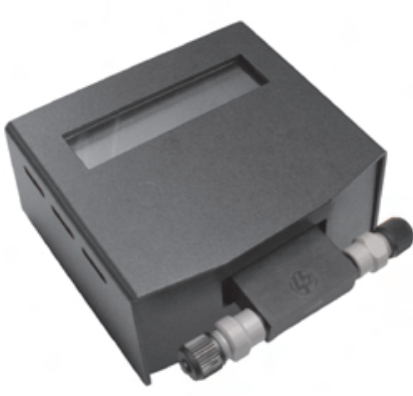

c)

Fig.1.4 Commercially available thermal flow sensors for measuring liquid flow in the nl-min ${ }^{-1}$ range, with a) $\mu$-flow digital mass flow meter for liquids by Bronkhorst Nederland B.V. [1.54], b) SLG 1430 liquid mass flow meter by Sensirion AG [1.56] and c) nano flow sensor by Upchurch Scientific [1.57]. 
is dependent on the radius $r$ of the capillary. Capillary radii range from $20 \mu \mathrm{m}$ to $200 \mu \mathrm{m}$, therefore the pressure drop over these micro sensors remains relatively small.

$$
\mathrm{R}_{\text {circ }}=\frac{8 \mu l}{\pi r^{4}}
$$

The micro flow sensor from Sensirion AG (Fig. 1.3b) and Upchurch Scientific (Fig. 1.3c) are based on MEMS technology. The Sensirion AG sensor applies a MEMS based sensor chip mounted on the capillary tube [1.56]. A similar approach is followed by Weiping et al. [1.58].

\subsection{Aim of the Research}

The problem that is investigated in this thesis is related to performance limitations of thermal micro flow sensors due to miniaturisation. Miniaturisation means that flow channel dimensions and flow rates become smaller. This requires thermally-isolated flow channels where the complete fluid can be heated in order to obtain maximum sensitivity. Furthermore, the pressure drop across the flow channels increases significantly with miniaturisation, where the best possible solution is to use circular flow channel cross-sections.

With miniaturisation also sensor elements become smaller. In current micro flow sensors these elements are made by metal thin films on top of thermally-isolated flow channels. The problem is that thin films reproduce poorly and that practically all materials properties are subject to drift. This drift and poor reproducibility translates directly into the accuracy of thermal micro flow sensors.

The work presented in this thesis aims at solving material drift problems by a combination of two innovations. One innovation is to use power control on heater elements. By continuously measuring the resistance value the dissipated power can be precisely controlled, while resistance value can drift over time. The other innovation relates to the use of a temperature-balancing control system in combination with a thermopile measuring a differential temperature. Of importance is that the thermopile has zero-offset, not giving an output voltage if the thermopile is at a uniform temperature. Alternatively a sensor resistor and heat waves can be used to provide for a low offset-drift error signal (Chap. 8).

\subsection{Outline of the Thesis}

The content of this thesis is largely based on published articles or conference articles to be published in literature, therefore each chapter can be read by itself. In the next chapter (Chap. 2) design aspects of low-drift micro flow sensors are discussed, focussing on drift influences and flow sensing concepts to compensate for 
drift. In the last chapter general conclusions are drawn, with recommendations for future research. The following sub-sections give a general outline of the research results set forth in this thesis.

\section{Chapter 3 - Surface Channel Technology}

In this chapter a microchannel fabrication concept is described, allowing for easy fluidic interfacing and integration of transducer material in close proximity to the fluid. This is achieved by the reliable fabrication of completely sealed microchannels directly below the substrate surface. The microchannels are subsequently released for thermal isolation. The viability of the concept was demonstrated by fabrication of several micro-fluidic device templates.

\section{Chapter 4 - Miniaturised Calorimetric Flow Sensor}

One of the device templates in Chap. 3 was applied in the fabrication of a calorimetric miniaturised flow sensor, with a linear sensor response measured for water flow up to flow rates in the order of $300 \mathrm{nl} \cdot \mathrm{min}^{-1}$. The realised flow sensor consists of a microchannel with low hydraulic resistance and $4.5 \mathrm{nl}$ total fluid volume. The sensor demonstrates the applicability of the microchannel technology for micro thermal flow sensor for measuring minute amounts of liquid flow.

\section{Chapter 5 - Low-Drift Flow Sensor with Thermopile-Based Power Feedback}

In this chapter a more advanced micro flow sensor is presented using two heaters and a thermopile in order to eliminate material drift. The low offset drift of the thermopile is exploited in a feedback loop controlling the dissipated powers in the heater resistors, minimising inevitable influences of resistance drift, mismatch of thinfilm metal resistors and thermopile material drift. The control system cancels the flow-induced temperature difference across the thermopile by controlling a power difference between both heater resistors, thereby giving a measure of the flow rate. The flow sensor was characterised for power difference versus water flow rates up to $1.5 \mu \mathrm{l} \cdot \mathrm{min}^{-1}$. It is demonstrated that material drift is largely compensated, however the sensor still suffers from externally applied temperature gradients over the chip.

\section{Chapter 6 - Low-Drift U-shaped Thermopile Flow Sensor}

To compensate for external temperature gradient a different sensor layout is investigated, comprising of a freely-suspended U-shaped microchannel with integrated thermopile. The structure is symmetrically heated by a heater at the top of the U-shape. The thermal imbalance caused by liquid flow is sensed by the thermopile. The U-shape microchannel facilitates the integration of a large number of 
thermocouple junctions, resulting in a highly-sensitive calorimetric flow sensor $\left(40 \mathrm{mV} / \mu \mathrm{l} \cdot \mathrm{min}^{-1}\right.$ at $2 \mathrm{~mW}$ heating power). Accurate measurements up to $400 \mathrm{nl} \cdot \mathrm{min}^{-1}$ water flow have been obtained applying a temperature-balancing control system.

\section{Chapter 7 - Ambient Temperature-Gradient Compensated Low-Drift Flow Sensor}

The U-shaped sensor presented in Chap. 6 shows a significant improvement in sensitivity, however a small dependence on external temperature gradients remains, caused by conduction through the surrounding air. In this chapter a special meandering layout of the microchannels is used, resulting in a fully symmetrical sensor. The thermopile junctions are placed, such that a fluid flow results in summation of thermopile voltages, while the influence of external temperature gradients is completely eliminated in the measured thermopile voltage.

\section{Chapter 8-AC-Driven Temperature-Balancing Flow Sensor with Power Feedback}

In this chapter another measurement technique is explored to realise a low-drift sensor using AC-driven heat waves. A simple sensor structure similar to the sensor presented in Chap. 4 is used, with three resistors on a suspended segment of the thermal-isolated flow channel. The outer resistors are heated by an alternating current, while the addition of the heat waves arriving at the centre resistor is measured. In this way, a drift performance similar to the thermopile-based sensors is obtained at the expense of a much slower response time. This is caused by the low operating frequency of the lock-in technique in order to detect the small signal amplitudes.

\section{Chapter 9 - Nano-Nozzle Electrospray Emitters Fabricated by a Micro- to Nano-Fluidic Via Technology}

In this chapter the surface channel technology is extended by the possibility to integrate nanochannels using a micro- to nano-fluidic via technology. The main advantage of the technology is the ability to position freely-suspended nanochannels anywhere on a micro-fluidic chip. Nano-nozzle electrospray emitters were fabricated using this process on freely-suspended microchannels. Leak-tight delivery of fluid from a fluidic reservoir was established through long microchannels. Stable electrospray IV-curves could be obtained from fabricated nano-electrospray emitters.

\section{References}

[1.1] F. Cascetta, "Short history of the flowmetering", IS A Trans., 34 (1995) 229-243.

[1.2] S. Haasl, G. Stemme, Y. Gianchandani, O. Tabata, H. Zappe, "Comprehensive microsystems", Elsevier (2007) chapter 2.07, "Flow sensors".

[1.3] N.T. Nguyen, "Micromachined flow sensors - a review", Flow Meas. Instrum., 8 (1997) 7-16. 
[1.4] P. Gravesen, J. Branebjerg, O.S. Jensen, "Microfluidics - a review", J. Micromech. Microeng., 3 (1993) 168-182.

[1.5] C. Darwin, "The origin of species, by means of natural selection", London, John Murray (1859) chapter XIII, "Classification - Morphology".

[1.6] M. Dijkstra, J.J. van Baar, R.J. Wiegerink, T.S.J. Lammerink, J.H. de Boer, G.J.M. Krijnen, "Artificial sensory hairs based on the flow sensitive receptor hairs of crickets, J. Micromech. Microeng., 15 (2005) S132-S138.

[1.7] G.J.M. Krijnen, J. Casas, "Imitating cricket mechanosensory hairs: dream or reality?", Proc. Sensors and Sensing in Biology and Engineering (2008).

[1.8] C.M. Bruinink, R.K. Jaganatharaja, M.J. de Boer, J.W. Berenschot, M.L. Kolster, T.S.J. Lammerink, R.J. Wiegerink, G.J.M. Krijnen, "Advancements in technology and design of biomimetic flow sensor arrays", Proc. IEEE MEMS (2009).

[1.9] R. Attia, D.C. Pregibon, P.S. Doyle, J-L. Viovy, D. Bartolo, "Soft microflow sensors", Lab Chip, 9 (2009) 1213-1218.

[1.10] U. Gebhard, H. Hein, E. Just, P. Ruther, "Combination of a fluidic micro-oscillator and micro-actuator in LIGA-technique for medical application", Proc. IEEE Transducers (1997).

[1.11] U. Gebhard, H. Hein, U.Schmidt, "Numerical investigation of fluidic microoscillators", J. Micromech. Microeng., 6 (1996) 115-117.

[1.12] E.W. Simões, R. Furlan, R.E.B. Leminski, M.R. Gongora-Rubio, M.T. Pereira, N.I. Morimoto, J.J. Santiago Avilés, "Microfluidic oscillator for gas flow control and measurement”, Flow Meas. Instrum., 16 (2005) 7-12.

[1.13] P.H. Wright, "The Coanda meter - a fluidic digital gas flowmeter", J. Phys. E: Sci. Instrum., 13 (1980) 433-436.

[1.14] R.F. Boucher, "Minimum flow optimization of fluidic flowmeters", Meas. Sci. Technol., 6 (1995) 872-879.

[1.15] R.E. Oosterbroek, T.S.J. Lammerink, J.W. Berenschot, G.J.M. Krijnen, M.C. Elwenspoek, A. van den Berg, "Micromachined pressure/flow-sensor", Sensor. Actuat. A-Phys., 77 (1999) 167-177.

[1.16] R.E. Oosterbroek, "Modeling, design and realization of microfluidic components", $\mathrm{Ph}$. D. thesis (1999), University of Twente, Enschede, The Netherlands.

[1.17] P. Enoksson, G. Stemme, E. Stemme, "A silicon resonant sensor structure for Coriolis mass-flow measurements”, J. Microelectromech. S., 6 (1997) 119-125.

[1.18] D. Sparks, R. Smith, M. Straayer, J. Cripe, R. Schneider, A. Chimbayo, S. Anasari, N. Najafi, "Measurement of density and chemical concentration using a microfluidic chip", Lab Chip, 3 (2003) 19-21.

[1.19] A. Mehendale, "Coriolis mass flow rate meters for low flows", Ph. D. thesis (2008), University of Twente, Enschede, The Netherlands.

[1.20] J. Haneveld, T.S.J. Lammerink, M. Dijkstra, H. Droogendijk, M.J. de Boer, R.J. Wiegerink, "Highly sensitive micro Coriolis mass flow sensor", Proc. IEEE MEMS (2008).

[1.21] J. Haneveld, T.S.J. Lammerink, M.J. de Boer, R.J. Wiegerink, "Micro Coriolis mass flow sensor with integrated capacitive readout", Proc. IEEE MEMS (2009). 
[1.22] M. Elwenspoek, R. Wiegerink, "Mechanical Microsensors”, Berlin Heidelberg, Springer (2001) chapter 8, "Flow sensors".

[1.23] B.R. Munson, D.F. Young, T.H. Okiishi, "Fundamentals of fluid mechanics", New York, John Wiley \& Sons, $4^{\text {th }}$ edition (2000) chapter 9, "Flow over immersed bodies".

[1.24] A. Bejan, "Heat Transfer", New York, John Wiley \& Sons (1993) chapter 5, "External forced convection".

[1.25] N. Damean, P.P.L. Regtien, M. Elwenspoek, "Heat transfer in a MEMS for microfluidics", Sensor. Actuat. A-Phys., 105 (2003) 137-149.

[1.26] B.W. van Oudheusden, "Silicon thermal flow sensor with a two-dimensional direction sensitivity”, Meas. Sci. Technol., 1 (1990) 565-575.

[1.27] K.A.A. Makinwa, J.H. Huijsing, "A wind-sensor interface using thermal sigma delta modulation techniques", Sensor. Actuat. A-Phys., 92 (2001) 280-285.

[1.28] S.P. Matova, "Calibration and testing of integrated thermal airflow sensors", Ph. D. thesis (2004), University Delft, Delft, The Netherlands.

[1.29] A. Glaninger, A. Jachimowicz, F. Kohl, R. Chabicovsky, G. Urban, "Wide range semiconductor flow sensor”, Sensor. Actuat. A-Phys., 85 (2000) 139-146.

[1.30] F. Kohl, R. Fasching, F. Keplinger, R. Chabicovsky, A. Jachimowicz, G. Urban, "Development of miniaturized semiconductor flow sensors", Measurement, 33 (2003) 109-119.

[1.31] S. Oda, M. Anzai, S. Uematsu, K. Watanabe "A silicon micromachined flow sensor using thermopiles for heat transfer measurements", IEEE Trans. Instrum. Meas., 52 (2003) 1155-1159.

[1.32] S-C. Roh, Y-M. Choi, S-Y. Kim, "Sensitivity enhancement of a silicon micromachined thermal flow sensor", Sensor. Actuat. A-Phys., 128 (2006) 1-6.

[1.33] Ch. Stamatopoulos, A. Petropoulos, D.S. Mathioulakis, G. Kaltsas, "Study of an integrated thermal sensor in different operational modes, under laminar, transitional and turbulent flow regimes", Exp. Therm Fluid Sci., 32 (2008) 1687-1693.

[1.34] D. Randjelovíc, A. Petropoulos, G. Kaltsas, M. Stojanovíc, Ž. Lavić, Z. Djurić, M. Matić, "Multipurpose MEMS thermal sensor based on thermopiles", Sensor. Actuat. A-Phys., 141 (2008) 404-413.

[1.35] M.J.A.M. van Putten, M.H.P.M. van Putten, A.F.P. van Putten, "High accurate flow measurements with thermal flow sensors using the alternating direction method", Proc. IEEE Instrumentation and Measurement (1996) 527-530.

[1.36] P. Brushi, A. Diligenti, D. Navarrini, M. Piotto, “A double heater integrated gas flow sensor with thermal feedback", Sensor. Actuat. A-Phys., 123-124 (2005) 210-215.

[1.37] P. Brushi, D. Navarrini, M. Piotto, "A close-loop mass flow controller based on static solid-state devices”, J. Microelectromech. Syst., 15 (2006) 652-658.

[1.38] T.S.J. Lammerink, N.R. Tas, G.J.M. Krijnen, M. Elwenpoek, “A new class of thermal flow sensors using $\Delta \mathrm{T}=0$ as a control signal", Proc. IEEE MEMS, Miyazaki, Japan (2000) 525-530.

[1.39] S. Billat, K. Kliche, R. Gronmaier, P. Nommensen, J. Auber, F. Hedrich, R. Zengerle, "Monolithic integration of micro-channel on disposable flow sensors for medical applications", Sensor. Actuat. A-Phys., 145-146 (2008) 66-74. 
[1.40] A. Rasmussen, C. Mavriplis, M.E. Zaghloul, O. Mikulchenko, K. Mayaram, "Simulation and optimization of a microfluidic flow sensor", Sensor. Actuat. A-Phys., 88 (2001) 121-132.

[1.41] J.J. van Baar., R.J. Wiegerink, T.S.J. Lammerink, G.J.M. Krijnen, M.C. Elwenspoek "Micromachined structures for thermal measurement of fluid and flow parameters", J. Micromech. Microeng., 11 (2001) 311-318.

[1.42] J.J. van Baar., "Distributed thermal micro sensors for fluid flow", Ph. D. thesis (2002), University of Twente, Enschede, The Netherlands.

[1.43] J.J. van Baar., W.A. Verweij, M. Dijkstra, R.J. Wiegerink, T.S.J. Lammerink, G.J.M. Krijnen, "Micromachined two dimensional resistor arrays for determination of gas parameters", Proc. IEEE Tranducers (2003) 1606-1609.

[1.44] E. Meng, P-Y. Li, Y-C. Tai, "A biocompatible Parylene thermal flow sensing array", Sensor. Actuat. A-Phys., 144 (2008) 18-28.

[1.45] E. Meng, "MEMS technology and devices for a micro fluid dosing system", Ph. D. thesis (2003) chapter 4, "A micro flow sensing array", California Institute of Technology, Pasadena, California (USA).

[1.46] A. Bejan, "Heat Transfer", New York, John Wiley \& Sons (1993) chapter 6, "Internal forced convection"

[1.47] Il.Y. Han, D-K. Kim, S.J. Kim, "Study on the transient characteristics of the sensor tube of a thermal mass flow meter", Int. J. Heat Mass Transfer, 48 (2005) 2583-2592.

[1.48] S. Wu, Q. Lin, Y. Yuen, Y-C. Tai, "MEMS flow sensors for nano-fluidic applications", Sensor. Actuat. A-Phys., 89 (2001) 152-158.

[1.49] Y. Mizuno, M. Liger, Y-C. Tai, "Nanofluidic flowmeter using carbon sensing element", Proc. IEEE MEMS (2004) 322-325.

[1.50] M. Dijkstra, T.S.J. Lammerink, R.J. Wiegerink, M. Elwenspoek, "Nano-flow thermal sensors applying dynamic $\omega-2 \omega$ sensing method", Proc. MME, (2006) 29-32.

[1.51] J. Xie, J. Shih, Y-C. Tai, "Integrated surface-micromachined mass flow controller", Proc. IEEE MEMS (2003).

[1.52] L. Schöler, B. Lange, K. Seibel, H. Schäfer, M. Walder, N. Freidrich, D. Ehrhardt, F. Schönfeld, G. Zech, M. Böhm, "Monolithically integrated micro flow sensors for lab-on-chip applications", Microelctron. Eng., 78-79 (2005) 164-170.

[1.53] J. Shih, Y-C. Tai, Y. Miao, T.D. Lee, "Microfabricated platform for nanoscale flow sensing and control", Proc. IEEE Sensors (2006) 1432-1435.

[1.54] Bronkhorst Nederland B.V., " $\mu$-flow - series L01 digital mass flow meters/controllers for liquids", Datasheet (2006).

[1.55] J. Lötters, "New generation of liquid flow sensors for the nanoliter through milliliter minute range with extremely small internal volume”, Proc. Sensors (2003) 1432-1435.

[1.56] Sensirion AG, "SLG 1430 - Liquid mass flow meter", Datasheet (2006).

[1.57] Upchurch Scientific, "Nano flow sensor", Catalogue of Chromatography \& Fluidic Components (2007-2008) 26-27.

[1.58] Y. Weiping, L. Chong, L. Jianhua, M. Lingzhi, N. Defang, "Thermal distribution microfluidic sensor based on silicon", Sensor. Actuat. B-Chem., 108 (2005) 943-946. 


\section{Low-Drift Flow Sensing}

This chapter discusses design aspect of low-drift micro flow sensors. The accuracy of thermal micro flow sensors is influenced by the degradation of thin-film resistors, measured as flicker and electromigration noise, resulting in long-term drift of the sensor output. This makes thin-film resistors unsuitable as accurate absolute temperature sensors for micro flow sensors in the nl.min-1 range. Power control and four-point contacts can be used to accurately dissipate heat in the thin-film resistors. Thermopiles can be applied to generate a voltage proportional to a temperature difference, where a thermopile at uniform temperature has zero-offset. Additionally, sensitivity drift of thermal flow sensors can be eliminated by using the temperature balancing concept, where a power difference provides a measure of the flow rate.

\subsection{Introduction}

Thin-film metal and thin-film semiconductor resistors have been used as heating elements and as temperature sensing elements in micromachined thermal flow sensors for the nl. $\mathrm{min}^{-1}$ range [2.1-2.4]. Fabrication is simple, requiring deposition and patterning of a single thin-film layer. However, the resistance value of thin-film resistors are subject to degradation mechanisms and reproduce poorly. This chapter discusses the degradation mechanisms of thin-film resistors, where it can be concluded that accurate micro thermal flow sensors cannot be obtained using thin-film resistors as absolute temperature sensors. Instead thermopiles can be used to measure a differential temperature [2.5-2.8], where no output voltage is generated if the thermopile is at a uniform temperature. The temperature-balancing flow sensing concept is explained where power control on heater resistors and a thermopile are used in combination with a temperature-balancing controller to obtain a micro thermal flow sensor with high-accuracy and low offset drift.

\subsection{Thin-Film Transducers}

\subsubsection{Thin-Film Resistors}

Thin-film resistors used as absolute temperature sensing elements in micromachined thermal flow sensors determine the temperature $\Delta T$ by measuring the resistance $R$ change with temperature (2.1), where $\alpha$ is the temperature dependency of resistivity and $R_{0}$ the resistance defined at the reference temperature $\Delta T=0$.

$$
\mathrm{R}=\mathrm{R}_{0}(1+\alpha \Delta T)
$$


A current flowing through the resistor is used to measure the voltage across the resistor and hence the resistance value is obtained. Additionally, the measured voltage contains Johnson noise, with power spectral density $S_{V}=4 k T R$, caused by thermal excitation of the charge carriers in the resistor, with $k$ the Boltzmann constant and $T$ the absolute temperature. Johnson or thermal noise is independent of frequency $f$. It determines the maximum precision in temperature $\Delta T$ obtained by averaging the resistance value of a resistor $\mathrm{R}$, with given temperature dependency of resistivity $\alpha$.

Materials in microengineering have many defects and irregularities at the microscopic level. Thin-film metal resistors are non-uniform and contain many grainboundaries. The quality of the thin-film directly relates to the impact of degradation mechanisms (e.g. oxidation) on thin-film properties [2.9]. The degradation is measured in the voltage across the resistive material as $1 / f$ or flicker noise, with a measurement current flowing through the resistor. The power spectral density $S_{V}$ follows Hooge's formula (2.2), with $V$ the voltage across the resistor, $N$ the number of charge carriers and $\gamma_{H}$ a constant related to the quality of the thin-film resistor. The constant $\gamma_{H}$ can depend weakly on temperature, with $\alpha$ between 0.7 and 1.3 [2.9].

$$
S_{V}=\frac{\gamma_{H} V^{2}}{N f^{\alpha}}
$$

Noise spectra with higher $\alpha$ relate to diffusion mechanisms along the resistor [2.10]. This form of degradation is exponentially dependent on temperature as it depends on a diffusion coefficient $D$. Electromigration takes place if additionally the power spectral density finds relation with the current density $j$, with $\alpha$ close to 2 [2.11-2.13]. Electromigration occurs by a flux of atoms $\vec{J}_{e m}(2.3)$ due to a driving force $\vec{F}$, which is caused by the electron wind and the charge on the atom resulting in an effective charge number $Z^{*}$, with $q$ the elementary charge and $\vec{E}$ the electrostatic field [2.14]. The flux by electromigration $\vec{J}_{e m}$ is opposed by a diffusion flux $\vec{J}_{\text {diff }}$ depending on the atom concentration $N$ and diffusion coefficient $D$, with activation energy $E_{a}(2.3)$.

$$
\vec{J}=\vec{J}_{\text {diff }}+\vec{J}_{e m}=-D \nabla N+\frac{N D}{k T} \vec{F} \quad D=D_{0} e^{-E_{a} / k T} \quad \vec{F}=Z^{*} q \vec{E}
$$

Figure 2.1 shows the power spectral density of an aluminium resistor where a $1 / f^{2}$ slope due to electromigration is apparent. Electromigration noise (2.4) relates to the flux $\vec{J}_{e m}$ by electromigration an is both dependent on the current density $j$ and on tem- 


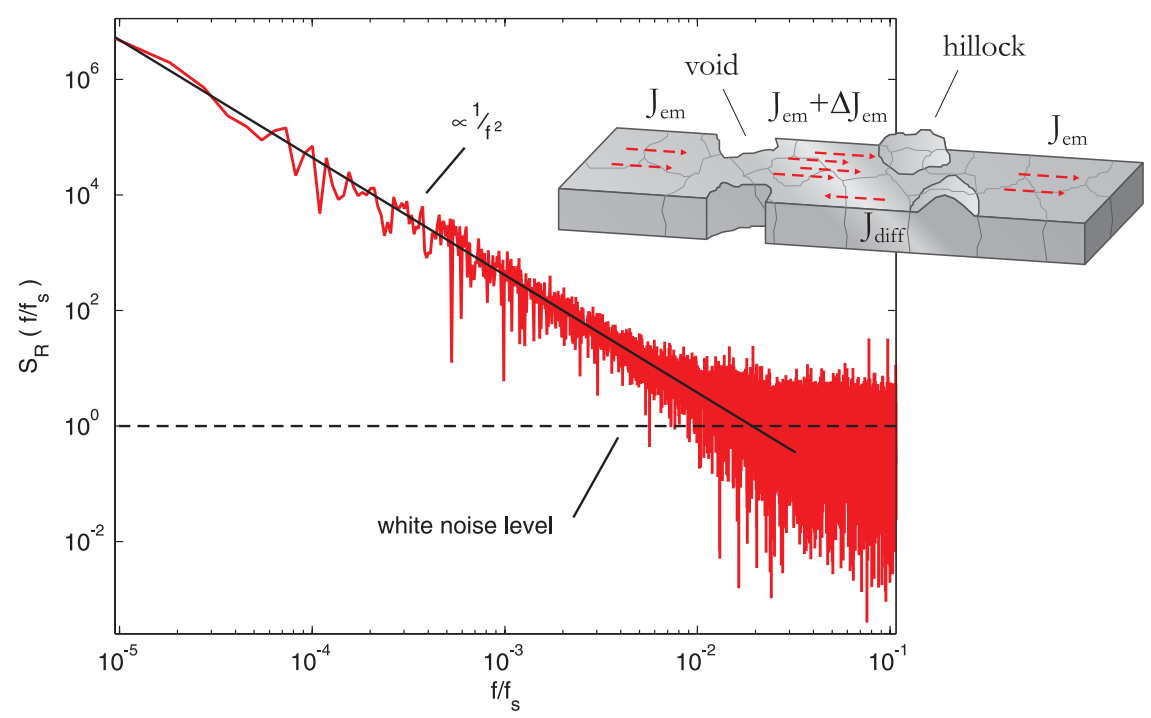

Fig. 2.1 Measured power spectral density of electromigration induced degradation (inset) in an aluminium resistor applying $6 \mathrm{~mA} \cdot \mathrm{um}^{-2}$ current density for 58 days at $0.04 \mathrm{~Hz}$ sampling frequency $\mathrm{fs}_{\mathrm{s}}$

perature $T$, where $\rho$ is the resistivity of the resistor and $\gamma$ a constant depending on the quality of the film [2.9].

$$
S_{R}=\frac{\gamma \rho j}{f^{\alpha} k T} e^{-E_{a} / k T}
$$

Local differences in the electromigration flux can cause the creation of voids and hillocks at high current densities (Fig. 2.1), which can alter the thermal profile on the resistor when used as heater. The increase in current density near voids can eventually lead to failure of the thin-film resistor. Electromigration mainly occurs along grain boundaries having lowest activation energy $E_{a}$, which explains the direct correlation of the grain-size distribution with the electromigration noise and reliability performance of the thin-film $[2.15,2.16]$. Annealing can be applied to increases grain size after recrystallisation, which can improve electromigration performance [2.17, 2.18].

Thin-film resistors used for temperature sensing are measured using small currents. The noise will therefore largely be consistent with flicker noise, in the absence of other diffusion mechanisms. The flicker noise affects the accuracy of flow sensors using thin-film resistors as temperature sensors. Thin-film resistors are noisier than bulk material, due to the thin-film microstructure and are therefore not accurate enough to be used in flow sensors in the $\mathrm{nl} \cdot \mathrm{min}^{-1}$ range. 


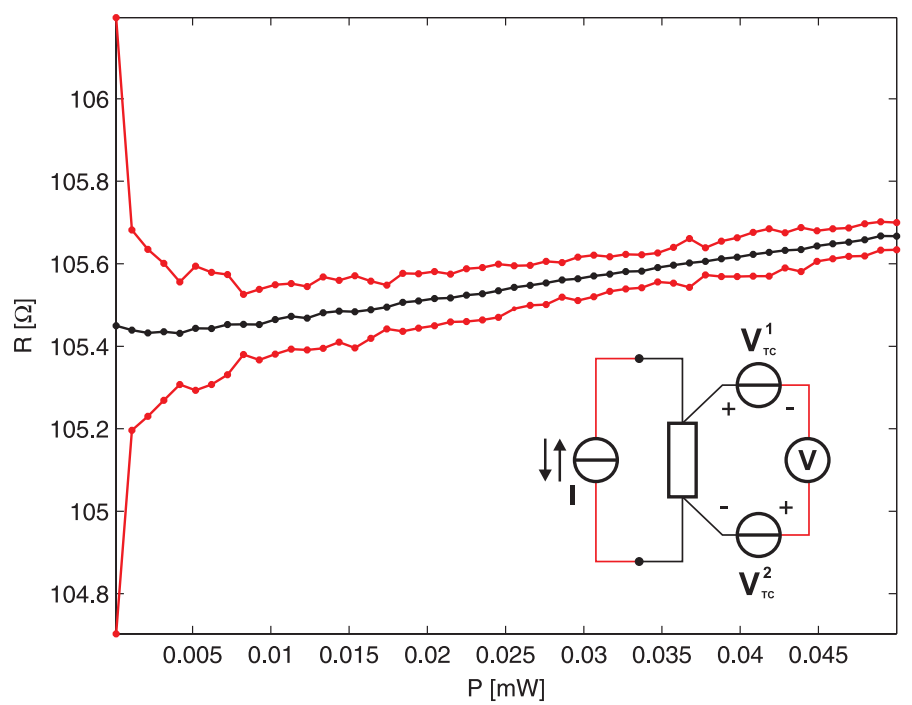

Fig. 2.2 Thermocouple offset compensation by alternating the direction of the current used during four-point measurement of the resistance value $\mathrm{R}$ at different dissipated powers $P$.

Thin-film resistors used for heating have high current densities at elevated temperatures. The noise will therefore depend on degradation by electromigration. Thin-film resistors can however still be used as heaters in thermal flow sensors for the nl. $\mathrm{min}^{-1}$ range. The dissipated heat can be controlled accurately by adjusting the current through the resistor according to $I=(P / R)^{-2}$, where the resistance has to be determined by four-point contact.

Controlling a small power in a heater resistor leads to a small voltage drop across the resistor. Thermocouple voltages $V_{T C}$, generated in contact leads (Fig. 2.2) between the voltmeter and the resistor, have therefore influence on the measured resistance value $R$. The effect of the thermocouple offset voltages can be eliminated by alternating the current $I$ through the resistor, where the resistance value $R$ is determined by averaging over the resistance values obtained with alternating directions of the heating current (Fig. 2.2).

\subsection{2, Thin-Film Thermopiles}

In leads of electrically conductive material a temperature gradient along the conducting lead gives rise to a thermo-diffusion current, which is additional to the current given by Ohm's law. Both currents must balance if the lead has open ends, meaning that no net current can flow through the lead. The thermo-diffusion current is proportional to the temperature gradient, which through the drift-current by Ohm's law results in an electrical potential being generated (Seebeck effect). The generated 
electrical potential can actually be measured with a thermopile, where leads of different material are connected in series, parallel to the direction of the temperature gradient $\Delta T_{T C}$ to be measured (Fig. 2.3).

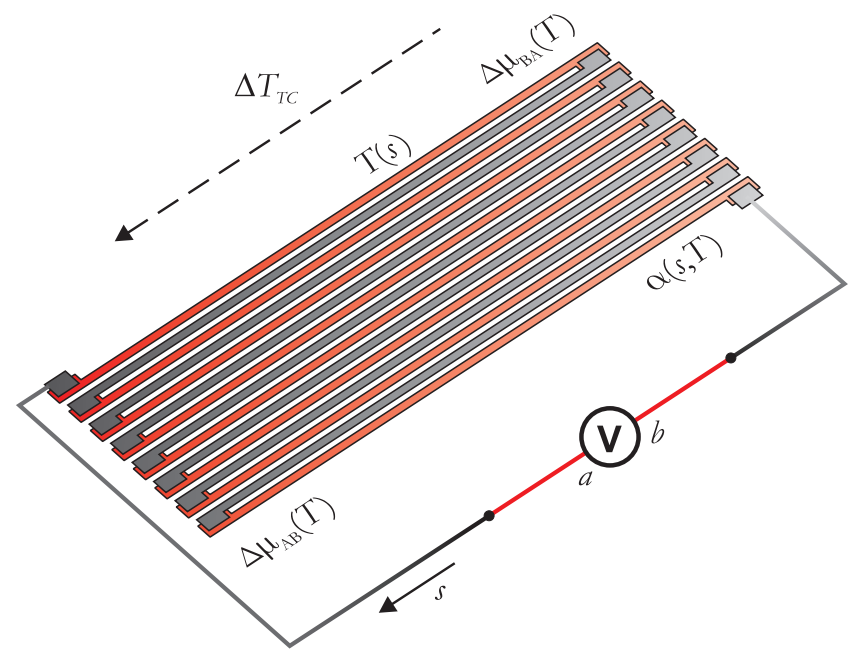

Fig. 2.3 Measurement of thermopile voltage $V_{\text {ab }}$ generated by a temperature gradient $\Delta T_{T C}$ along thermopile leads of different conductive materials.

A complete description of thermal-electrical conduction in conductive materials is gained considering the interrelation of the heat flux $\vec{b}$ with the electrical current density $\vec{j}$. Equations (2.5) describe the transport of charge carriers, which depends on the temperature gradient $\nabla T$ in the material and the electrochemical field $\vec{E}_{e-c b}=\vec{E}-\nabla \mu / q$, which is the combination of the electrostatic field $\vec{E}$ and the gradient in the chemical potential $\mu$ [2.19]. Equations (2.5) describe the Seebeck, Peltier and Thomson effects by a matrix $L$, where $L^{11}$ is the electrical conductivity $\sigma$ of the material.

$$
\begin{aligned}
& \vec{j}=L^{11} \vec{E}_{e-c b}-L^{12} \nabla T \\
& \vec{b}=L^{21} \vec{E}_{e-c b}-L^{22} \nabla T
\end{aligned}
$$

A voltage $V_{a b}$ can be measured due to the aforementioned Seebeck effect, which is generated by the temperature gradient $\nabla T(s)$ along leads of different material. The voltage is generated without a current density $\vec{j}$ flowing through the material. This means that the electrochemical field $\vec{E}_{e-c h}$ can be derived from the equation for $\vec{j}$ in (2.5), which can then be substituted in the equation for $\vec{b}$ in (2.5). This results in the equation for the electrochemical field $\vec{E}_{e-c b}$ in (2.6), generated by a temperature 
gradient, where $\alpha$ is the Seebeck coefficient of the material. The other equation in (2.6) gives the heat flux $\vec{b}$ through the material due to the temperature gradient, with $\kappa$ the thermal conductivity, which for metals is approximately equal to $L^{22}$, defined as $L^{22}=L T \sigma$ according to the Wiedemann-Franz law, where $L$ is the Lorentz number and $T$ the absolute temperature [2.19].

$$
\begin{aligned}
& \vec{E}_{e-c h}=\frac{L^{12}}{L^{11}} \nabla T=\alpha \nabla T \\
& \vec{b}=-\left(L^{22}-L^{21}\left(L^{11}\right)^{-1} L^{12}\right) \nabla T=-\kappa \nabla T
\end{aligned}
$$

The voltage measured $V_{a b}$ on a thermopile equals the line integral over the equation for the electrochemical field $\vec{E}_{e-c b}$ in (2.6) taken along the electrical path connecting a voltmeter end terminal at $a$ to an end terminal at $b$ (Fig. 2.3). The line integral (2.7) is written in terms of the electrostatic field $\vec{E}$, the chemical potential $\mu$, the effective Seebeck coefficient $\alpha_{\text {eff }}$ evaluated over the cross-section of the lead [2.20] and the temperature $T$ along the lead.

$$
\begin{gathered}
\int_{a}^{b} \vec{E} \cdot d \vec{s}-\frac{1}{q} \int_{a}^{b} \partial_{s} \mu(s, T) d s=\int_{a}^{b} \alpha_{e f f}(s, T) \partial_{s} T(s) d s \Rightarrow \\
V_{a b}=-\int_{a}^{b} \alpha_{e f f}(s, T) \partial_{s} T(s) d s
\end{gathered}
$$

The term for the chemical potential $\mu$ in (2.7) does not contribute to the measured voltage, assuming that both end terminals are of the same material at the same temperature (the same $\mu$ ), and that the voltmeter has high impedance [2.19]. It also means that the measured voltage $V$ is independent of contact potentials between materials with different chemical potential, e.g. the thermocouple junctions $\Delta \mu_{A B}$ in Fig. 2.3. The term with the temperature gradient in (2.7) gives a measurable voltage only when the Seebeck coefficient varies along the line integral, which is the case for a thermopile made of leads of different material. A thermopile has a drift-free zero offset, in that no voltage is generated if the thermopile is at a uniform temperature.

A figure of merit (2.8) for thermocouple materials determines the signal-to-noise ratio $[2.21,2.22]$, where the thermal conductivity $\kappa$ should be low allowing for the largest possible temperature difference between the thermopile junctions. The Seebeck coefficient $\alpha$ should be high to have high output voltage and the electrical resistivity $\rho$ should be low to have low Johnson noise.

$$
z=\frac{\alpha^{2}}{\rho \kappa}
$$


The figure of merit is high for materials with large Seebeck coefficient, which for semiconductors is larger than for metals. High output voltage is obtained by combining materials with a large difference in Seebeck coefficient, which can be obtained with metal-semiconductor thermocouples. For instance, in this thesis aluminium with a Seebeck coefficient of $\alpha_{A l}=-1.66 \mu \mathrm{V} \cdot \mathrm{K}^{-1}$ [2.23] has been used in combination with boron doped poly-Si with approximately a Seebeck coefficient of $\alpha_{\text {poly-Si }}=0.6 \mathrm{mV} \cdot \mathrm{K}^{-1}$ [2.22] at a measured resistivity of $\rho_{\text {poy }-\mathrm{Si}}=0.7 \mathrm{~m} \Omega \cdot \mathrm{m}^{-1}$. Many different semiconductor materials, with high Seebeck coefficient are commonly used, like bismuth-telluride and indium-arsenide [2.23], while $\mathrm{Al} /$ poly-Si ${ }^{++}$thermopiles can easily be fabricated with standard CMOS technology.

\subsection{Flow Sensing Concepts}

\subsubsection{Constant-Power Calorimetric Sensing with Resistors}

A thermally fully heated calorimetric flow sensor requires the integration of resistors for heating and temperature sensing on a freely suspended flow channel (Fig. 2.4). Most calorimetric flow sensors use constant heating power on a heater resistor centred between two sensing resistors. Constant power usually means that a
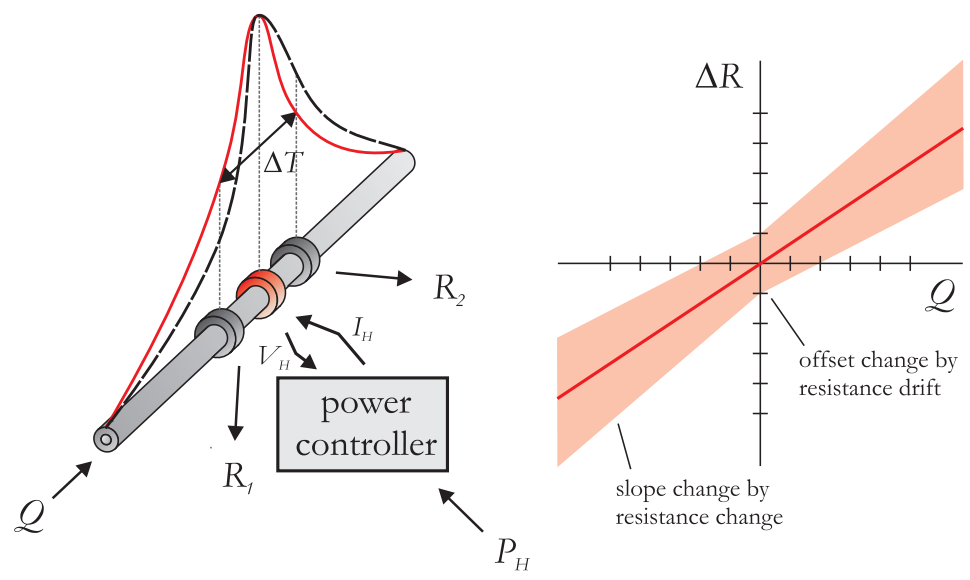

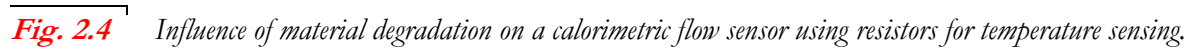

fixed current is applied. However, accurate micro thermal flow sensors require fourpoint measurement of the heater resistor and power control of the heating power $P_{H}$ in order to at least eliminate the influence of resistance drift on the sensor output. Four-point resistance measurement can be applied, while alternating the measurement current (Fig. 2.2) in order to accurately obtain the resistance value of the heater. A 
power controller uses the measured resistance value to control the current required to dissipate the exact heating power required. The temperature imbalance due to the flow rate $Q$ is measured by two resistors $R_{1}, R_{2}$, which can still cause long-term drift in the sensor output, where a change in offset in the sensor output is caused by a difference in resistance drift between both sensor resistors (Fig. 2.4). A change in slope and offset is caused by drift in the temperature coefficient of resistivity of the resistors.

Figure 2.5 shows thermal model results evaluating drift influences on a calorimetric flow sensor using Pt resistors (Chap. 4), where the temperature imbalance due to the flow rate $Q$ is measured by a voltage difference $\Delta V$ output of a Wheatstrone bridge containing the sensing resistors $R_{1}, R_{2}$. It is assumed that drift due to heater resistor degradation is fully eliminated. Already a $1 \%$ increase in the resistance value $R_{0,1}$ causes a significant offset drift compared to the fully symmetrical flow sensor without offset. The offset is relatively large compared to the small change in resistance due to the flow, because of a small temperature coefficient of resistivity. An exaggerated $20 \%$ increase in the temperature coefficient of resistivity also causes an offset, while simultaneously the sensitivity for the flow rate $Q$ changes. The modelled drift in material properties indicate that, because of long-term drift in resistance values, thinfilm resistors cannot be used as absolute temperature sensors for accurate micro thermal flow sensing.

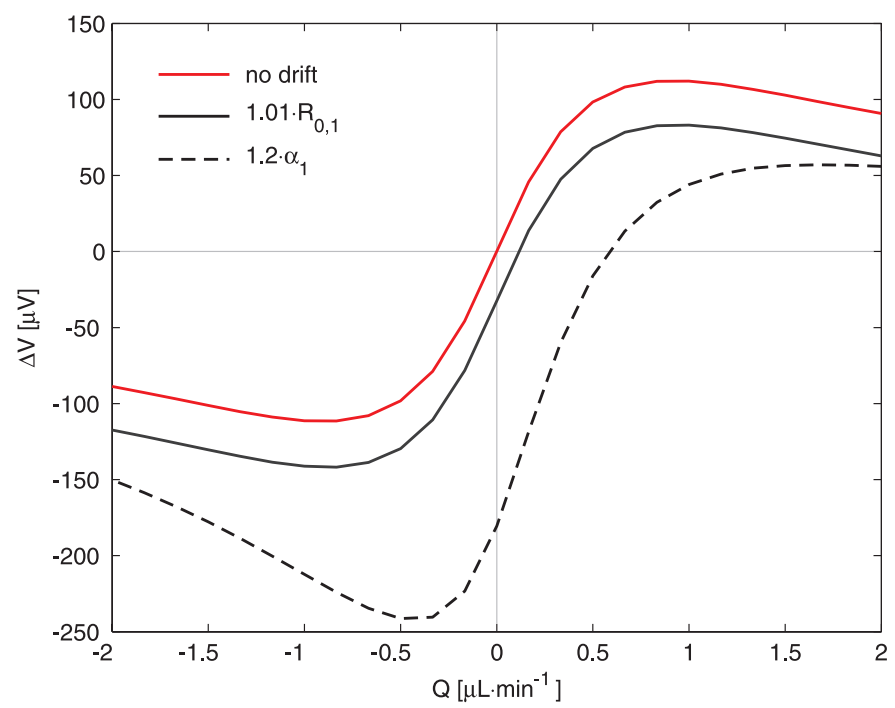

Fig. 2.5 Modelled influence of material degradation on a calorimetric flow sensor using resistors (Chap. 4), with $1.9 \mathrm{~mW}$ constant-power applied. 


\subsubsection{Constant-Power Calorimetric Sensing with Thermopiles}

The use of resistors as absolute temperature sensors can be avoided by using a thermopile (Fig. 2.6), with the thermocouple leads positioned parallel to the flow channel. Four-point resistance measurement with power control can be applied on resistors used for heating, cancelling the influence of resistance drift on the sensor output signal. A measure of the flow rate is given by the thermopile output voltage $V_{T C}$, generated by a temperature difference $\Delta T_{T C}$ with the two heaters dissipating equal heating power $P_{H}$. The voltage output $V_{T C}$ is obtained by integrating over the gradient in the thermal profile along the leads of the thermopile (2.7). Uniform drift in the Seebeck coefficient in one of the materials of the thermopile causes a change in the slope of the sensor output, because the thermopile changes its overall sensitivity. Non-uniform drift in the profile of the Seebeck coefficient along the thermopile leads $[2.24,2.25]$ can result in voltage offset drift even with a symmetrical temperature profile on the leads, with zero temperature difference $\Delta T_{T C}$ between the upstream and downstream thermopile junctions.

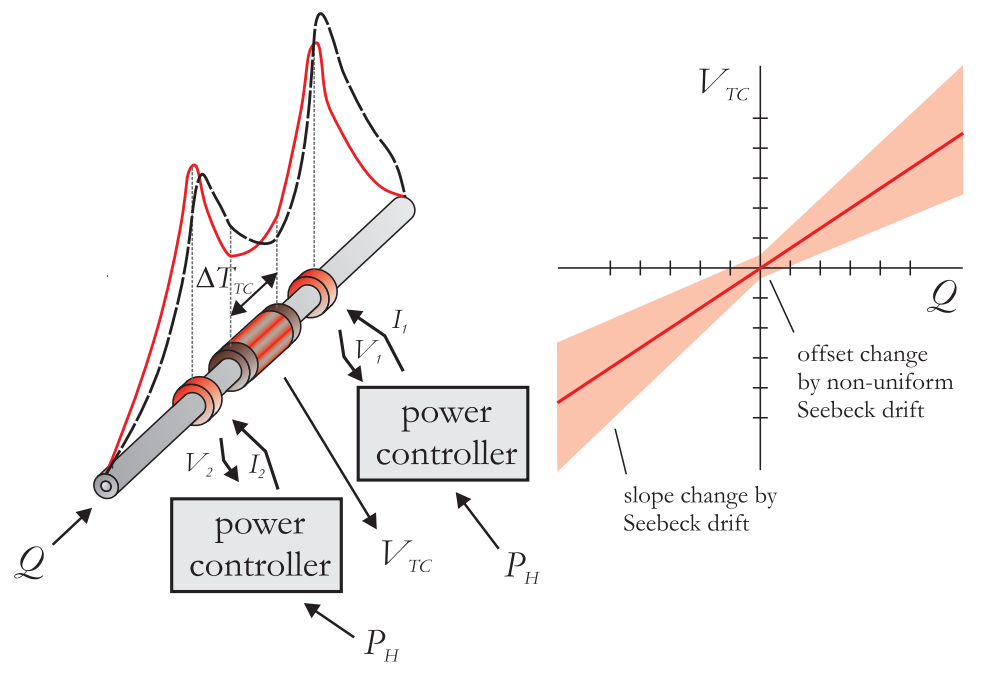

Fig.2.6 Influence of material degradation on a thermopile calorimetric flow sensor.

Figure 2.7 shows thermal model results evaluating drift influences on a calorimetric flow sensor applying an $\mathrm{Al} /$ poly-Si+ $\mathrm{Si}^{++}$thermopile (Chap. 5). The thermopile output voltage $\Delta V_{\text {TC }}$ gives a measure of the flow rate $Q$, with two heaters dissipating an equal amount of power $P_{H}$. A $50 \%$ overall change in the Seebeck coefficient of the doped poly-Si leads in the thermopile causes an increase in the output sensitivity of the flow 


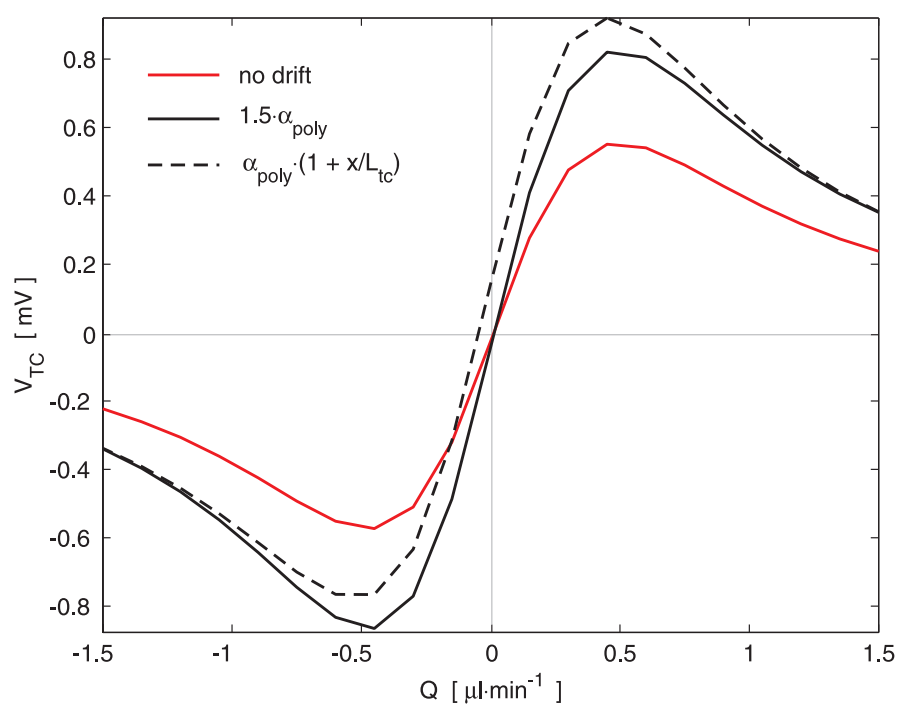

Fig. 2.7 Modelled influence of material degradation on a calorimetric flow sensor using a thermopile (Chap. 5), with $50 \mu W$ constant-power applied in each heater resistor.

sensor, without introducing offset drift. An exaggerated linear increasing Seebeck coefficient along the doped poly-Si leads of the thermopile results in an increase in sensor output sensitivity and the sensor output also obtains a small offset voltage. Long-term drift of the Seebeck coefficient of the thermocouple material, due to degradation, affects mostly the sensitivity of the calorimetric thermopile flow sensor. Non-uniform introduction of impurities and defects along the thermopile leads can result in a small drift in offset voltage.

\subsubsection{Temperature-Balancing Calorimetric Sensing with Thermopiles}

The calorimetric thermopile flow sensor can be made independent of thermopile sensitivity drift and thermopile non-linear characteristics applying a control-system feedback loop cancelling the generated output voltage $V_{T C}$. This provides for an almost drift-free error signal (Fig. 2.8). The output voltage $V_{T C}$ and temperature difference across the thermopile is cancelled by controlling a power difference $\Delta P$ between the the heater resistors using a balancing controller, with a fixed amount of total power $P_{T}$ being dissipated, according to the temperature-balancing anemometry principle $[2.26,2.27]$. Additionally, power controllers are used to control the powers $P_{1}, P_{2}$ dissipated in the heater resistors up- and downstream from the thermopile, where the heater resistance values are determined by four-point resistance measurements, cancelling influence of heater resistance drift. Applying a fluid flow 


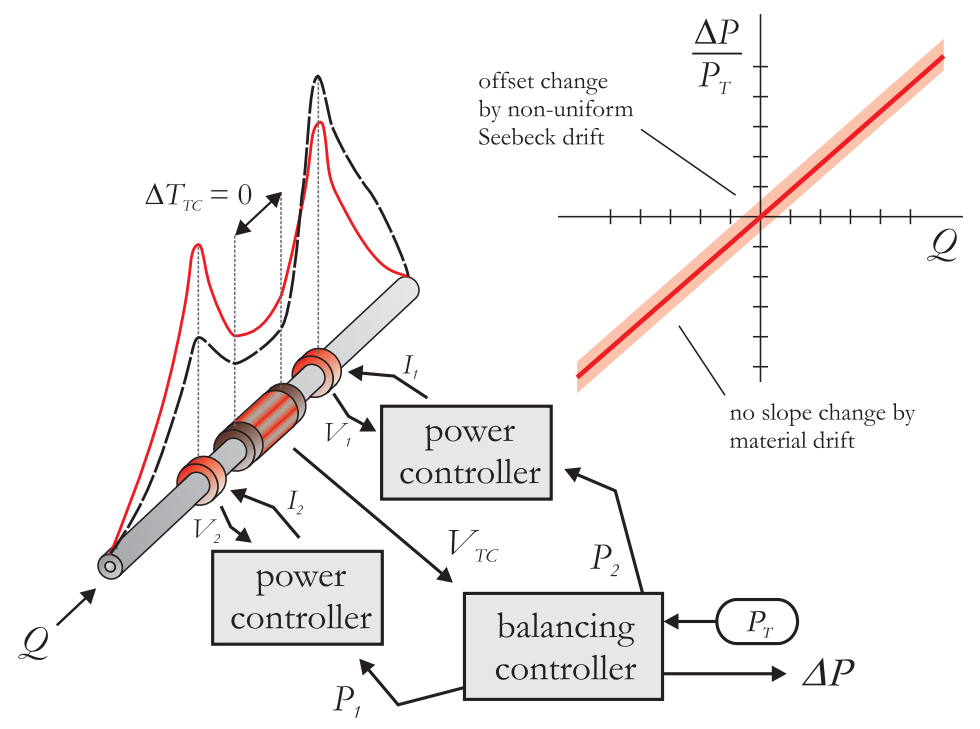

Fig. 2.8 Influence of material drift on thermopile-based temperature-balancing flow sensor.

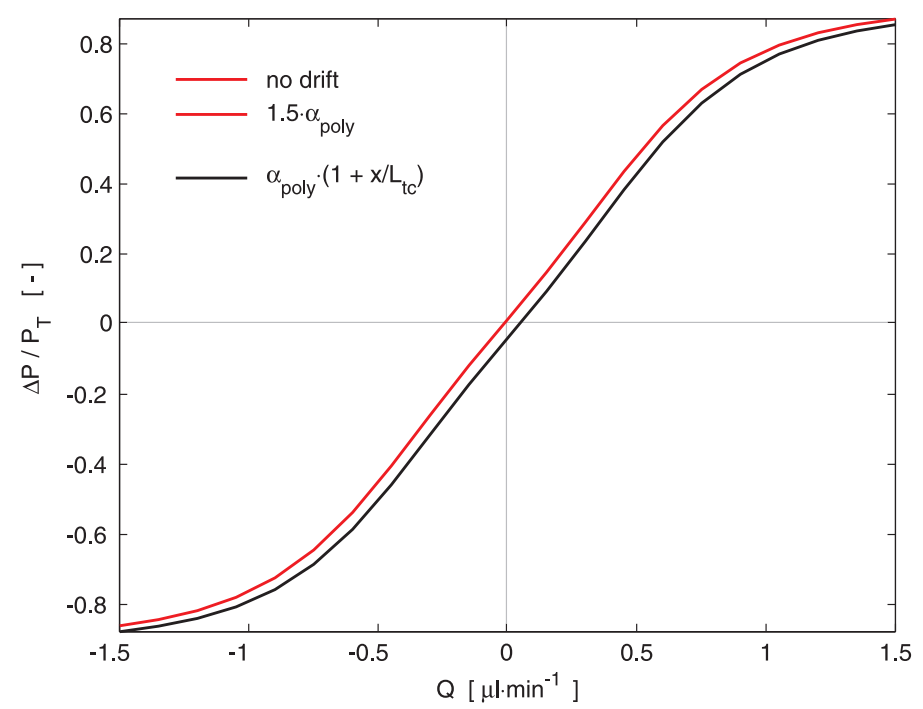

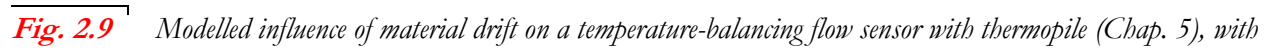
$0.1 \mathrm{~mW}$ total power dissipated. 
changes the temperature distribution around the heaters by advection of heat. This results in the relation $\Delta P / P_{T}$ being dependent on the flow rate $Q$, which is linear for small flow rates $Q$.

Figure 2.9 shows thermal model results evaluating drift influences on a calorimetric micro flow sensor using a thermopile (Chap. 5), while applying the temperaturebalancing anemometry concept. The sensitivity drift observed in open-loop (Fig. 2.7) is fully compensated. The sensor output also increases monotonically over a larger flow range. A linear increasing Seebeck coefficient along the doped poly-Si leads of the thermopile can still result in a minor offset voltage. Recalibration of the flow sensor can readily be performed by measuring the offset voltage without flow.

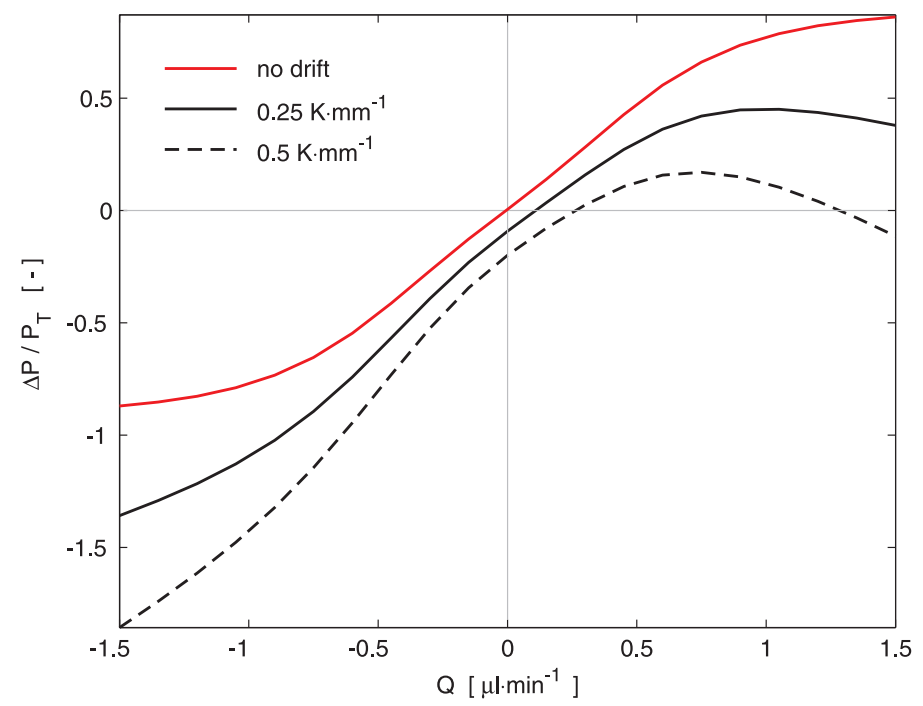

Fig. 2.10 Modelled influence of an external temperature gradient along the flow channel of a temperature-balancing flow sensor with thermopile (Chap. 5), with $0.1 \mathrm{~mW}$ total power dissipated.

Calorimetric flow sensors measure a flow induced temperature imbalance, which inadvertently means that the sensor also measures external temperature gradient in the direction of the flow. Figure 2.10 shows thermal model results, where the sensor output shows drift due to an external temperature gradient imposed on the freelysuspended flow channel. Multiple channels with flow in opposite direction can be used to compensate for these external temperature gradients (Chap. 8).

\section{References}

[2.1] S. Wu, Q. Lin, Y. Yuen, Y-C. Tai, "MEMS flow sensors for nano-fluidic applications", Sensor. Actuat. A-Phys., 89 (2001) 152-158. 
[2.2] Y. Mizuno, M. Liger, Y-C. Tai, "Nanofluidic flowmeter using carbon sensing element", Proc. IEEE MEMS (2004) 322-325.

[2.3] M. Dijkstra, T.S.J. Lammerink, R.J. Wiegerink, M. Elwenspoek, "Nano-flow thermal sensors applying dynamic $\omega-2 \omega$ sensing method", Proc. MME, (2006) 29-32.

[2.4] J. Xie, J. Shih, Y-C. Tai, "Integrated surface-micromachined mass flow controller", Proc. IEEE MEMS (2003).

[2.5] S-C. Roh, Y-M. Choi, S-Y. Kim, "Sensitivity enhancement of a silicon micromachined thermal flow sensor", Sensor. Actuat. A-Phys., 128 (2006) 1-6.

[2.6] T.H. Kim, S.J. Kim, "Development of a micro-thermal flow sensor with thin-film thermocouples", J. Micromech. Microeng., 16 (2006) 2502-2508.

[2.7] D. Randjelović, A. Petropoulos, G. Kaltsas, M. Stojanović, Z. Lazić, Z. Djurić, M. Matić, "Multipurpose MEMS thermal sensor based on thermopiles", Sens. Actuat. A-Phys., 141 (2008) 404-413.

[2.8] Ch. Stamatopoulos, A. Petropoulos, D.S. Mathioulakis, G. Kaltsas, "Study of an integrated thermal sensor in different operational modes, under laminar, transitional and turbulent flow regimes", Exp. Therm. Fluid Sci., 32 (2008) 1687-1693.

[2.9] C. Ciofi, B. Neri, "Low-frequency noise measurements as a characterization tool for degradation phenomena in solid-state devices", J. Phys. D: Appl. Phys., 33 (2000) R199-R216.

[2.10] Z. Moktadir, J. W. van Honschoten, M. Elwenspoek, "Long range diffusion noise in platinum microwires with metallic adhesion layers", Appl. Phys. Lett., 90 (2007) 233506.

[2.11] A. Bora, A.K. Raychaudhuri, "Evolution of $1 / \mathrm{f}^{\alpha}$ noise during electromigration stressing of metal film: Spectral signature of electromigration process", J. Appl. Phys., 99 (2006) 113701.

[2.12] J. Guo, B.K. Jones, G. Trefan, "The excess noise in integrated circuit interconnects before and after electromigration damage", Microelectron. Reliab., 39 (1999) 1677-1690.

[2.13] Z Çelik-Butler, W. Yang, "Characterization of electromigration parameters in VLSI metallizations by 1/f noise measurements", Solid State Electron, 34 (1991) 185-188.

[2.14] G.L. Baldini, I. de Munari, A. Scorzoni, F. Fantini, "Electromigration in thin-films for microelectronics", Microelectron. Reliab., 33 (1993) 1779-1805.

[2.15] C. Ciofi,. A. Diligenti, F. Giacomozzi, A. Nannini, B. Neri, "Low frequency electromigration noise and film microstructure in $\mathrm{Al} / \mathrm{Si}$ stripes: Electrical Measurements and TEM analysis", J. Electron. Mater., 22 (1993).

[2.16] S. di Pascoli, G. Iannaccone, "Noise and reliability in simulated thin metal films", Microelectron. Reliab., 48 (2008) 1015-1020.

[2.17] D.P. Field,. D. Dornisch, H.H. Tong, "Investigating the microstructure-reliability relationschip in Cu damascene lines", Scripta Mater., 45 (2001) 1069-1075.

[2.18] K. Maekawa,. K. Mori, N. Suzumura, K. Honda, Y. Hirose, K. Asai, A. Uedono, M. Kojima, "Impact of $\mathrm{Al}$ in $\mathrm{Cu}$ alloy interconnects on electro and stress migration reliabilities", Microelectron. Eng., 85 (2008) 2137-2141.

[2.19] N. W. Ashcroft, N. D. Mermin, "Solid State Physics", United States of America, Harcourt (1976) chapter 13, "The Semiclassical Theory of Conduction in Metals". 
[2.20] A.W. Herwaarden, "The Seebeck effect in silicon ICs", Sens. Actuat., 6 (1984) 245-254.

[2.21] G.C.M. Meijer, A.W. van Herwaarden, "Thermal Sensors", Bristol, Institute of Physics Publishing (1994).

[2.22] S. van Herwaarden, "Thermal vacuum sensors based on integrated silicon thermopiles", Ph. D. thesis (1987), University Delft, Delft, The Netherlands.

[2.23] D. Rowe, "CRC handbook of thermoelectrics", United States of America, CRC Press (1995).

[2.24] F. Jahan, M. Ballico "Overcoming inhomogeneity and hysteresis limitations of type R thermocouples in an international comparison", Int. J. Thermophys., 28 (2007) 1832-1842.

[2.25] M. Holmsten, J. Ivarsson, R. Falk, M. Lidbeck, L-E. Josefson "Inhomogeneity measurement of long thermocouples using short movable heating zone", Int. J. Thermophys., 29 (2008) 915-925.

[2.26] T.S.J. Lammerink, N.R. Tas, G.J.M. Krijnen, M. Elwenpoek, “A new class of thermal flow sensors using $\Delta \mathrm{T}=0$ as a control signal", Proc. IEEE MEMS, Miyazaki, Japan (2000) 525-530.

[2.27] P. Bruschi, A. Diligente, D. Navarrini, M. Piotto, "A double heater integrated gas flow sensor with thermal feedback", Sensor. Actuat. A-Phys., 123-124 (2005) 210-215. 


\section{Surface Channel Technology}

MEMS fluidic devices often require the integration of transducers with freely suspended microchannels. This chapter presents a microchannel fabrication concept, allowing for easy fluidic interfacing and integration of thin-film transducer material in close proximity to the fluid. This is achieved by the reliable fabrication of completely sealed microchannels directly below the substrate surface. The resulting planar surface allows for the deposition of transducer material and pattern transfer by lithography. The microchannels are subsequently released and fluidic entrance boles are created, while transducer material can be protected by photoresist. The viability of the concept was demonstrated by fabrication of several monolithic micro-fluidic device templates. Fabricated surface microchannel devices can be packaged or optionally be vacuum sealed using a glass capping wafer, which is under investigation at the time of writing. ${ }^{3.1}$

\subsection{Introduction}

The downscaling of microfluidic applications and devices requires the fabrication of small microchannels with the ability of integrating various types of transducers. Often the transducer needs to be integrated in close proximity to the fluid. For instance, in thermal applications the fluid should be heated or the fluid temperature has to be measured. Moreover, thermal isolation from the heat-conducting substrate requires the ability for the microchannels to be suspended freely [3.2-3.4].

Freely-suspended microchannels are also being used for fluid-mechanical resonance applications [3.5-3.13], requiring mechanically-strong microchannels, preventing buckling of the channels due to bending, but sufficiently compliant for actuation.

Microchannels have to be mechanically strong for high pressure applications, with proper fluidic interfacing to macro-fluidic connections, possibly with zero dead volume, while the interfacing to the microchannel and the microchannel itself should be chemically inert for various chemical applications [3.14-3.16].

Microchannels fabricated by bulk-bond micromachining and surface micromachining are limited in fulfilling mentioned requirements. For instance, integration of transducers in close proximity to the fluid is usually difficult for freely suspended microchannels fabricated using bulk micromachining and high-temperature bonding

3.1 This chapter is based on an article published in J. Micromech. Microeng. [3.1]. Contents has been added on experimental fluidic resonance measurements (Sec. 3.4.1) and packaging (Sec. 3.2.5, Sec. 3.4.3). 
techniques. Silicon fusion bonding has successfully been used by Enoksson et al. $[3.5,3.6]$ in defining freely-suspended microchannels (Fig. 3.1a) for a Coriolis massflow sensor. However, integration of capacitive read-out electrodes and packaging requires bonding of additional process wafers, leading to large wafer-stack implementations [3.7-3.9]. Smaller cross-section freely-suspended microchannels were made by Sparks, Tadigadapa et al. [3.10, 3.11] for fluid density and Coriolis mass flow sensing. The devices are fabricated using doping as etch stop and lost wafer processing of two silicon wafers with a glass wafer as support (Fig. 3.1b). Similarly, doping and etching of a Pyrex wafer is used by Agah, Lu et al. [3.14, 3.15] for micro gas-chromatography column fabrication (Fig. 3.1c). The microchannels are thermally isolated from the silicon by backside etching of the support wafer.
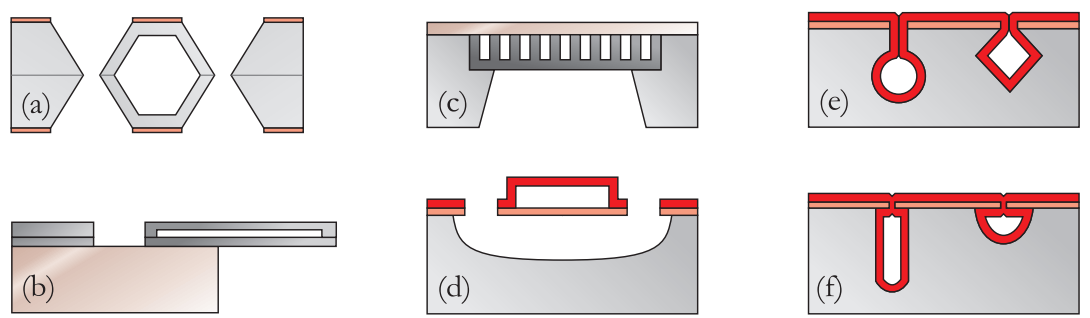

Fig. 3.1 Overview of possible freely suspended microchannel fabrication schemes, with a) wafer bonding by Enoksson et al. [3.5], b) lost-wafer process by Sparks et al. [3.10], c) doping etch stop and Pyrex wafer etching by Agah et al. [3.14], d) freely-suspended sacrificial microchannels, e) buried channel technology (BCT) by de Boer et al. [3.21] and f) embedded Parylene-microchannel technology by Chen et al. [3.24].

Surface micromachined channels (Fig. 3.1d) generally allow for easy integration of transducers on a single wafer [3.3,3.4], however step-coverage problems can occur for thin-film transducer material deposited across the microchannel. Furthermore, release of the sacrificial layer is time consuming, while wet-etching requires inert transducer materials for the etchant used. A major disadvantage of surface micromachined channels is the large hydraulic resistance, due to the limitation in channel height, determined by the sacrificial layer.

A good alternative is to make microchannels directly below the substrate surface [3.16-3.27]. This chapter describes the development of a versatile technological concept entitled surface channel technology (SCT), which is based on buried channel technology (BCT) (Fig. 3.1e) developed by de Boer et al. [3.21]. The BCT process has been applied in the fabrication of chromatography columns [3.22] (Fig. 3.2a) and micropipettes (Fig. 3.2b) for studying the mechanical properties of single DNA molecules [3.23]. Surface channel technology (SCT) combines freely-suspended low-stress microchannels directly below the substrate surface, with excellent properties for chemical-inert fluidic interfacing and integration of transducers in close 


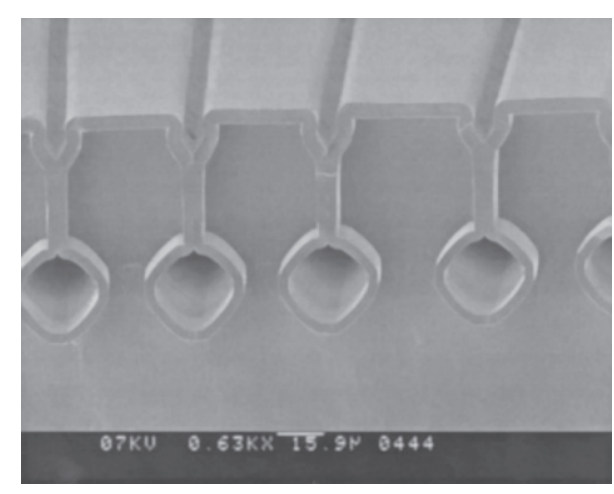

a)

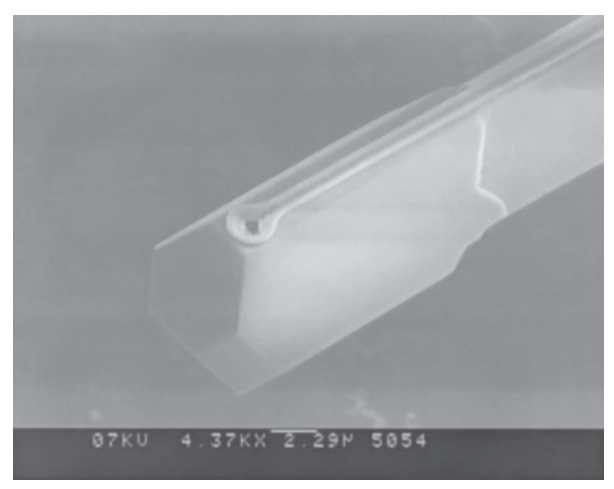

b)

Fig. 3.2 Devices fabricated with the buried channel technology, with a) a chromatography column and b) a micropipette, by de Boer et al. [3.21].

proximity to the fluid. A similar embedded parylene-microchannel technology (Fig. 3.1f) has recently been developed by Chen et al. [3.24]. The technology is compatible with CMOS back-end and further low-temperature MEMS processes, which enables the fabrication of embedded structures integrated with on-chip transducers for miniaturised lab-on-a-chip systems. A number of microfluidic devices have been fabricated with the embedded parylene technology [3.25-3.27].

\subsection{Surface Microchannel Concept}

The principle behind surface channel technology (SCT) is to make low-stress chemical-inert microchannels directly below the substrate surface, which are completely sealed after processing, leaving a planar substrate with negligible surface topology. This allows for the deposition of transducer materials and the pattern transfer by lithography, without material being deposited inside the microchannels. Subsequently the microchannels are released and fluidic entrance holes are created. Additionally, the microfluidic devices can be packaged and possibly vacuum sealed using a glass capping wafer.

\subsubsection{Surface Microchannel Fabrication}

Surface microchannels are fabricated (App. A.1) using a one-mask process scheme (Fig. 3.3). The mask layout defines etch holes, $2 \mu \mathrm{m}$ in width, combined into segmented lines, where each line defines a microchannel. The mask layout is transferred into a $500 \mathrm{~nm}$ tensile low-stress ( $<300 \mathrm{MPa}$ ) SiRN layer [3.28] deposited on a silicon wafer by LPCVD (Fig. 3.3a). The channels are isotropically etched 

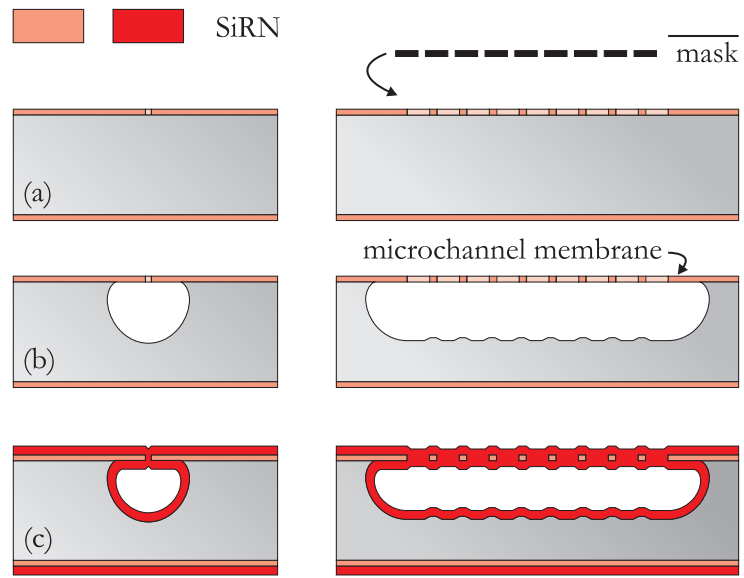

Fig. 3.3 Surface microchannel process scheme, applying a single mask defining etch boles in a $500 \mathrm{~nm}$ SiRN layer, showing perpendicular (left column) and longitudinal (right column) cross sections through the microchannel.

through the etch holes, leading to coalescence of neighbouring etch surfaces, resulting in long channels with semicircular profiles (Fig. 3.3b).

Applying etch holes in a slightly tensile SiRN layer ensures mechanical stability of the SiRN microchannel membranes formed during etching. This means that the etch holes can be properly sealed by a second LPCVD SiRN deposition (Fig. 3.3c). The inside surfaces of the microchannels are uniformly coated by SiRN during the deposition, where the thickness is limited by the sealing of the etch holes.

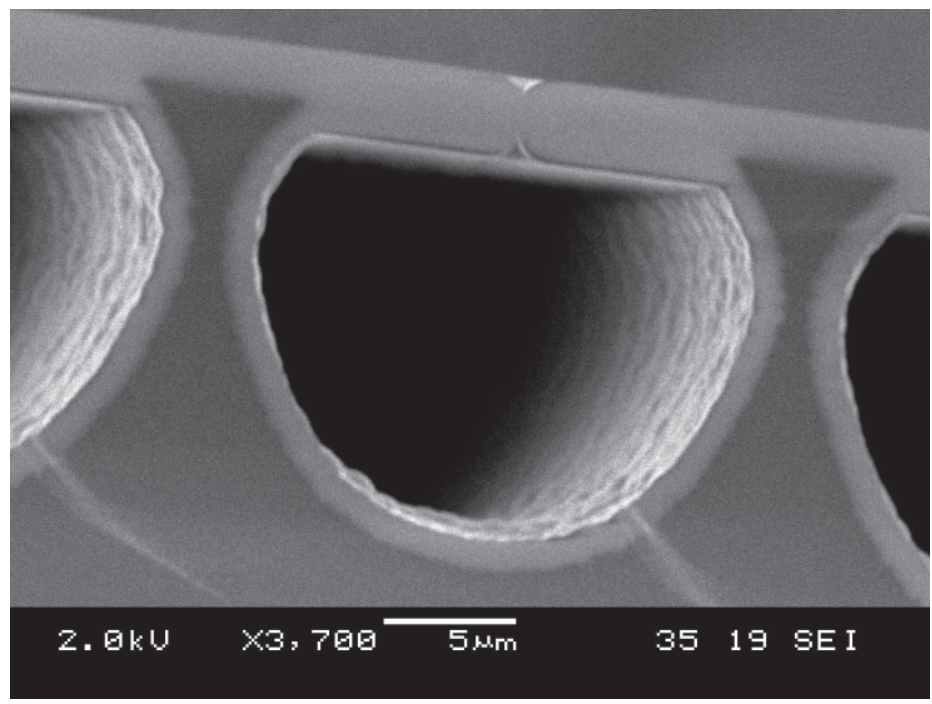

Fig. 3.4 Cross-section of sealed surface microchannels. 
A nearly perfect transfer of the mask layout into the initial SiRN layer is necessary, and high etching selectivity of Si compared to SiRN is required during microchannel etching, in order to limit the SiRN layer thickness required to seal the etch holes to about $2 \mu \mathrm{m}$, limiting internal stress in the layer. This is achieved using a $50 \mathrm{~nm} \mathrm{Cr}$ mask during microchannel etching. UV-ozone treatment is required, to improve wetting of the photoresist during wet-etching of the Cr mask layer.

Figure 3.4 shows a cross-section of sealed microchannels. The etch holes in the $500 \mathrm{~nm}$ SiRN layer were $2.3 \mu \mathrm{m}$ in width after SiRN etching and were perfectly sealed by the LPCVD deposition of a $1.3 \mu \mathrm{m}$ thick SiRN layer, leaving a planar wafer surface.

Figure 3.5 shows the formation of surface microchannels due to the coalescence of etch surfaces, leaving notches on the microchannel wall. Surface microchannels of varying diameter can be created, but the notch pattern and lithographic resolution puts a limit to the smallest channel diameter feasible.

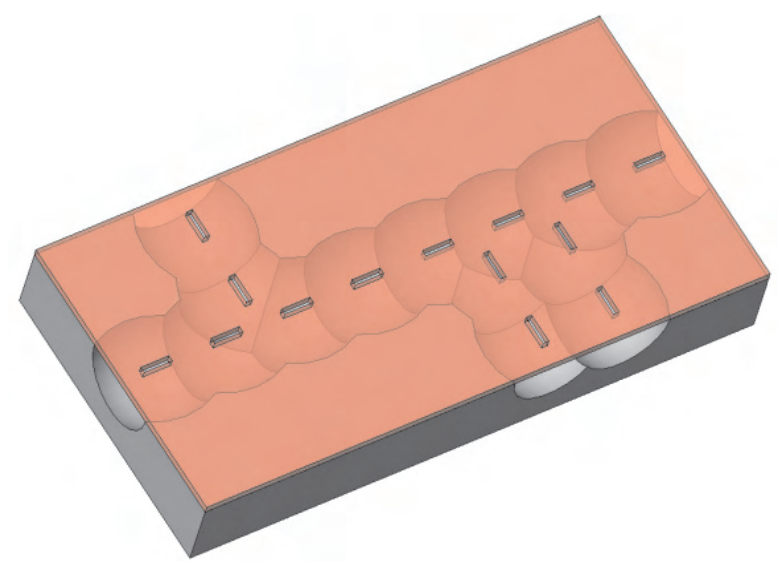

Fig. 3.5 Surface microchannels formed by the coalescence of etch surfaces.

The arrangement of etch holes determines the final size and shape of the microchannel network, independent of crystallographic orientation, giving large freedom in designing merged parallel channels, cavities and microchannel junctions. Etch-hole junctions and corners are to be avoided, because more SiRN would need to be deposited for proper sealing of the etch holes.

Etch holes were designed with a fixed width of $2 \mu \mathrm{m}$, which is still reproducible using contact lithography. The etch-hole length and separation length between etch holes were varied from $2 \mu \mathrm{m}$ up to $15 \mu \mathrm{m}$. The most reproducible microchannels with sufficient mechanical stability of the microchannel membrane were obtained at $5 \mu \mathrm{m}$ etch-hole length and $3 \mu \mathrm{m}$ separation between etch holes. 


\subsubsection{Transducer and Surface Micromachining Integration}

The sealed SiRN microchannel membranes are mechanically strong allowing for further processing, whereby thin-film transducers and surface micromachined structures can be integrated on top of the microchannel membranes. Figure 3.6 shows integration of various structures on top of the sealed microchannels, implemented in microfluidic devices described in Chap. 4-7, 9. The SEM micrograph in Fig. 3.6 shows the integration of $200 \mathrm{~nm}$ Pt structures, without adhesion layer, on top of several merged surface microchannels. The Pt structures were fabricated by sputtering, with proper step-coverage across sealed etch holes (Chap. 4). Several electrical resistance measurements of $4 \mu \mathrm{m}$ wires crossing etch holes $6 \mu \mathrm{m}$ in width did not show any electrical failures, confirming that standard fabrication processes can readily be applied for the integration of thin-film layers. The microchannels have sufficient mechanical stability, such that $\mathrm{Al} /$ poly-Si ${ }^{++}$thermopiles (App A.2, Fig. 3.6), requiring solid-source diffusion (SSD) at $1050{ }^{\circ} \mathrm{C}$ (Chap. 5-7), and even poly-Si sacrificial nanochannels (Chap. 9) can be created on top of the microchannel membranes.

\subsubsection{Surface Microchannel Release}

Surface microchannels are ready to be released after transducers have been fabri-

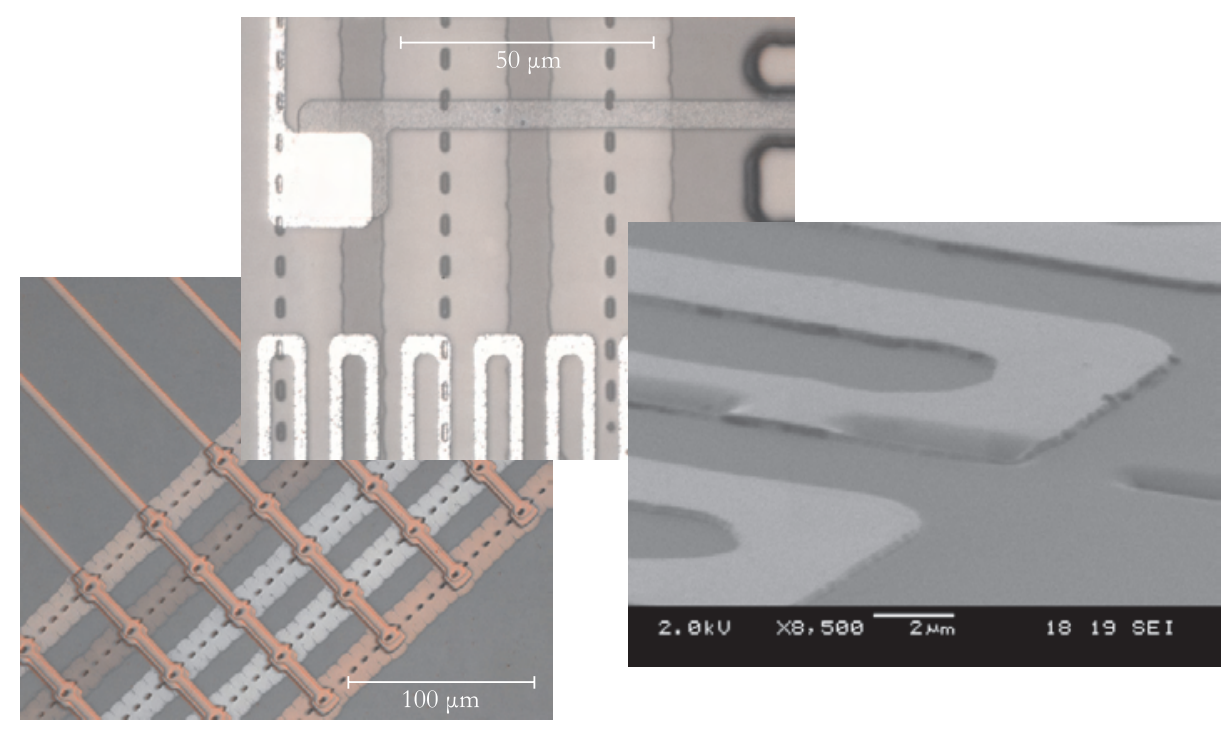

Fig. 3.6 Integration of various thin-film transducers and surface micromachined nanochannels on top of surface microchannels. 
cated Figure 3.7 depicts two possible process schemes. $\mathrm{KOH}$ or TMAH wet-etching or non-directional plasma etching can be used for the release of the surface microchannels. Both release methods require the definition of access windows to the bulk silicon (Fig. 3.7a), while fluidic entrance holes are defined in the same mask as the access windows to the silicon substrate and are etched through the SiRN layer simultaneously. The diameter of the etched microchannel should not exceed the edge of the access window or otherwise the microchannel is opened on the side during SiRN etching of the access windows. The spacing between access window and microchannel can be minimised by accurate determination of the microchannel diameter during etching.

Isolation cavities are etched, with $\mathrm{KOH}$ or TMAH, underneath the microchannels, provided that the microchannels are designed not to be aligned to the $<110>$-direction (Fig. 3.7b). Release by wet etching is straightforward, however the etch solution can be aggressive against desired transducer materials and the microchannels need to be cleaned, in order to remove remaining etch solution inside the microchannels after processing.

An alternative is the release of surface microchannels by non-directional plasma etching (Fig. 3.7c), which allows for the protection of transducer material by a thick photoresist layer, where the photoresist layer is removed after etching (Fig. 3.7d). This makes it possible to integrate any required type of transducer material.
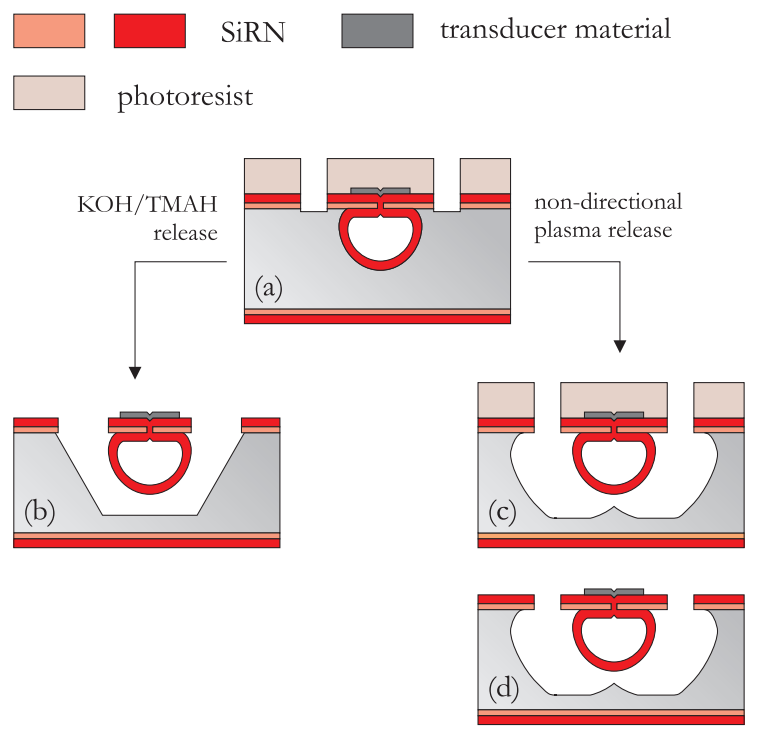

Fig. 3.7 Process schemes for the release of surface microchannels. 


\subsubsection{Fluidic Entrance Holes to the Surface Microchannels}

Fluidic entrance holes to the microchannel can be made on the frontside of the wafer, with minimum processing effort required (Fig. 3.8). The fluidic entrance holes have to be defined in the same photoresist mask containing the access windows, because it is the last lithography step possible on a planar substrate and because etch-
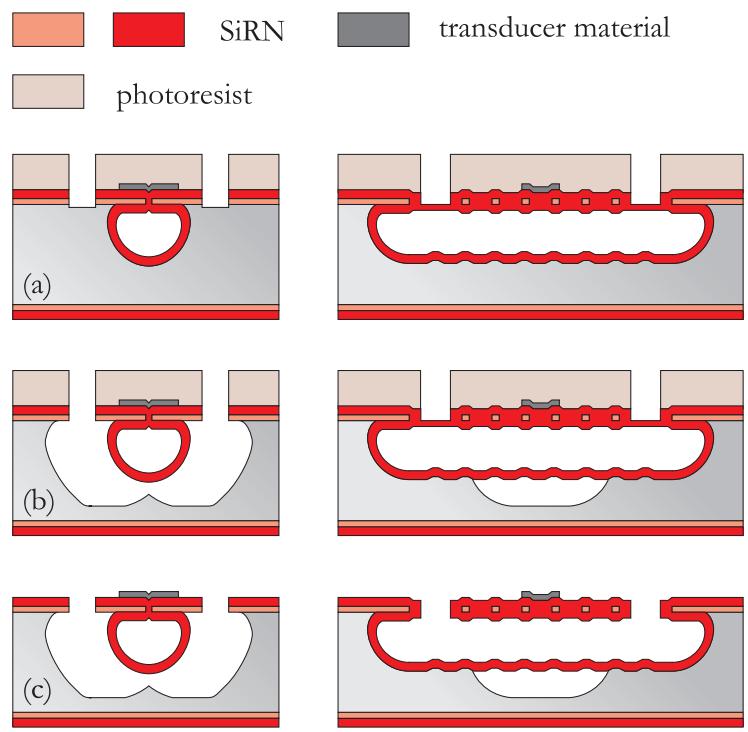

Fig. 3.8 Process scheme for the fabrication of fluidic entrance holes to the surface microchannels, showing perpendicular (left column) and longitudinal (right column) cross sections through the microchannel.

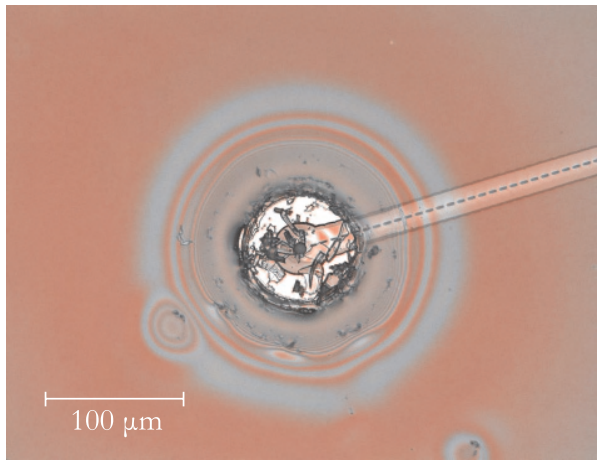

a)

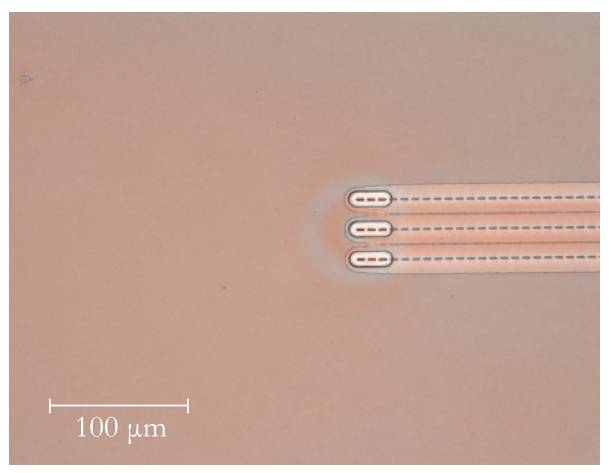

b)

Fig. 3.9 Mask transfer in photoresist with a) fluidic entrance boles defined in microchannel mask and b) fluidic entrance holes defined in access window mask. 
ing of fluidic entrance holes prior to the release of the microchannels causes the problem of improper mask transfer due to photoresist flowing into the microchannels (Fig. 3.9a).

However, the access windows to the silicon can be opened, while keeping the microchannels closed. This is possible, because the microchannel membranes are thicker than the SiRN layer on the substrate (Figs. 3.8a, 3.9b). This can be used for the protection of the inside surfaces of the microchannels during plasma release of the channels (Fig. 3.8b). The method can only be applied when the photoresist mask is not being removed, so that the fluidic entrance holes can be opened completely after the release of the microchannels (Fig. 3.8b), after which the photoresist can be completely removed (Fig. 3.8c).

Figure 3.10 shows separate fluidic entrance holes to several merged microchannels. The entrance holes are separated to maintain mechanical stability of the microchannel membrane. Chemically inert interfacing to the microchannel is possible, because fluid passing through the surface microchannels remains in contact with SiRN. Direct connection to the fluidic entrance holes can be made, applying fluidic connectors (e.g. Upchurch Scientific ${ }^{\mathbb{B}}-\mathrm{NanoPort}^{\mathrm{TM}}$ assemblies) or O-rings on top of the substrate. Haneveld et al. [3.29] showed that with additional backside processing chemical inert fluidic connections can be made from the backside of the wafer.

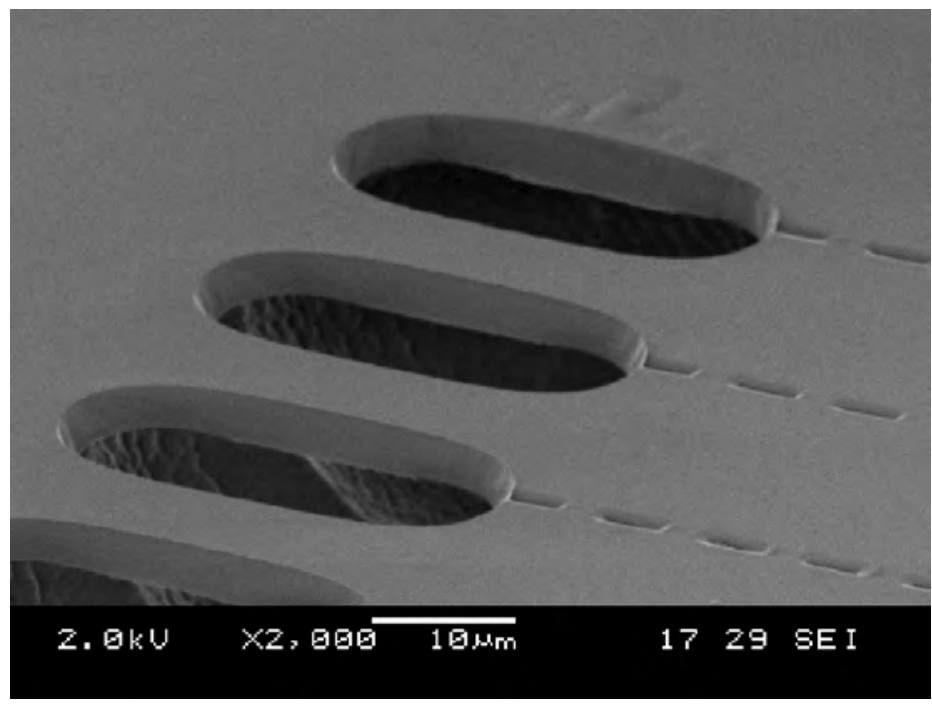

Fig. 3.10 Surface microchannel fluidic entrance holes, etched through the microchannel membrane. 


\subsubsection{Packaging of Surface Microchannel Devices}

Fabricated surface microchannel devices are susceptible to dust contamination after devices leave the cleanroom. Component-level packaging [3.30] can be applied to keep devices dust free. However, a wafer-level vacuum packaging solution can additionally reduce thermal loss from the microchannel to the surrounding silicon, increasing thermal flow sensor sensitivity and can increase Q-factors for fluidic resonance devices [3.31]. Wafer-level vacuum packaging solutions include the sealing of thin-shell vacuum capsules sealed by LPCVD deposition (e.g. [3.32]). Bartek et al. [3.33] showed that high vacuum, with pressures down to $10^{-3} \mathrm{~Pa}$, can be obtained by sealing capsules with an evaporated metal seal, where the sealing material is not being deposited inside the capsule. Burg et al. [3.31] achieved wafer-level vacuum packaging of microchannels fabricated below the wafer surface using glass-frit bonding of a Pyrex capping wafer, where electrical contact leads were fed through the glass-frit layer.

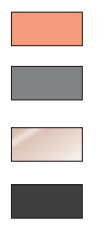

$$
\begin{aligned}
& \text { SiRN } \\
& \text { electrical contact lead } \\
& \text { glass substrate } \\
& \text { evaporated metal seal }
\end{aligned}
$$

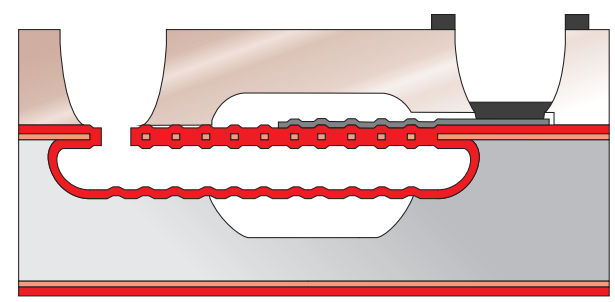

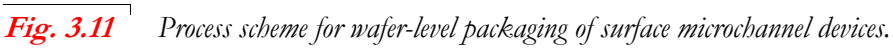

Microfluidic devices fabricated with surface channel technology are created using a single wafer. However, the surface of the wafer containing the microchannels is flat, also between etch holes, which means that the microchannel membranes can be properly bonded to a glass wafer, without having leakage through micro-cavities along the microchannels. This allows for the wafer-level packaging and possible vacuum sealing of surface channel devices by anodic bonding of a glass capping wafer containing vacuum cavities (Fig. 3.11). Electrical contact leads are contained in nanocavities etched in the capping wafer and are contacted by wafer-through contact holes etched through the wafer [3.34]. This allows for the anodic bonding of the glasswafer before vacuum sealing, such that oxygen formed during bonding [3.35] can be pumped out of the vacuum cavity, before sealing the cavity with an evaporated metal seal. In this way high-vacuum can be obtained [3.33]. The evaporated metal seal also functions as an electrical feed-through. 


\subsection{Channel Profile Etching}

An isotropic etch process is required for the fabrication of the microchannels, aiming for good channel uniformity across the wafer and good selectivity against SiRN. Either $\mathrm{HF} / \mathrm{HNO}_{3}$ wet-chemical etching or non-directional plasma etching can be used.

Wet-chemical etching using $\mathrm{HF} / \mathrm{HNO}_{3}$ has a significant loading effect on microchannel uniformity, requiring compensation structures [3.22]. Selectivity against
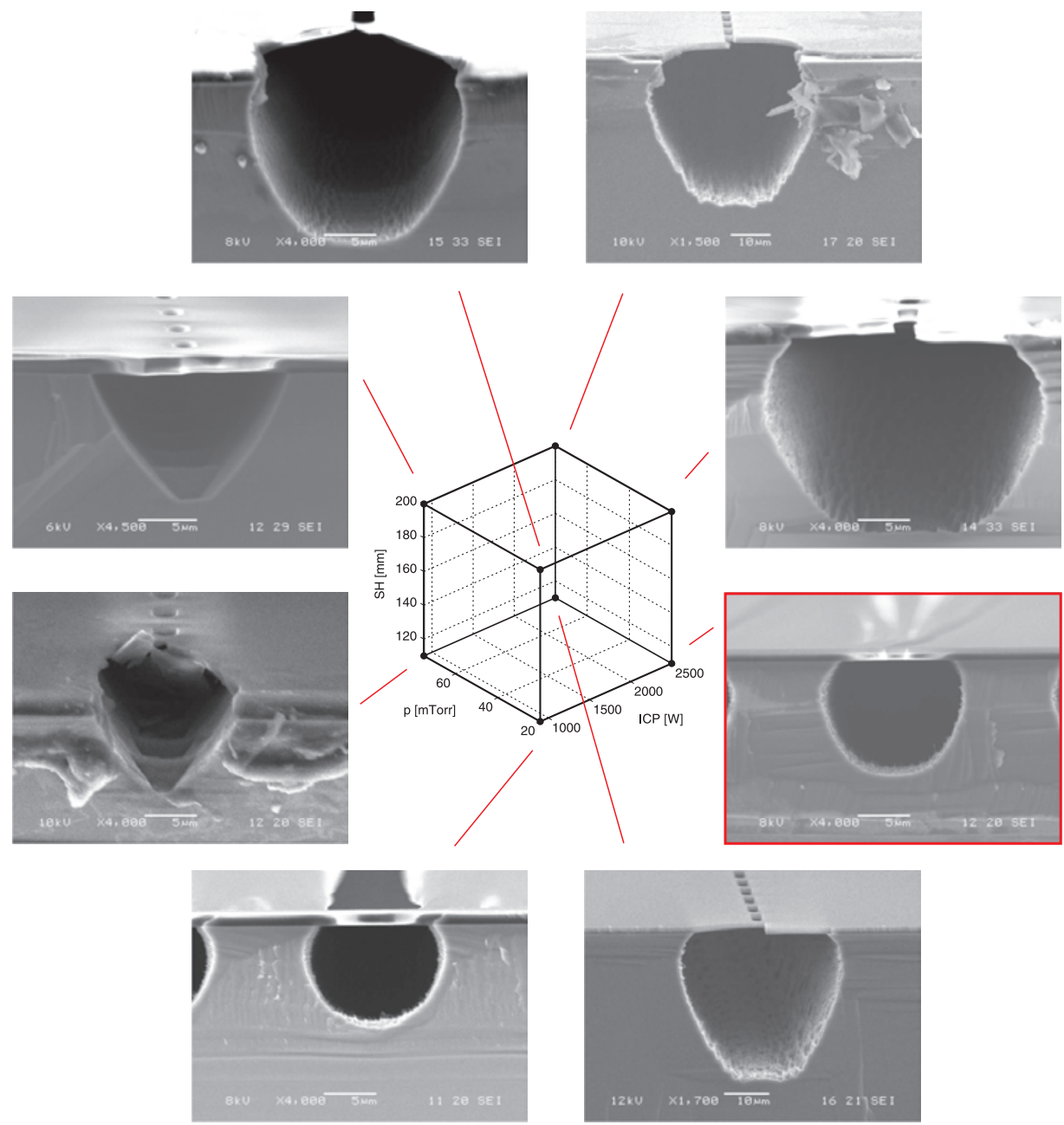

Fig. 3.12 Channel-profile parameter study using Adixen AMS 100 SE, applying fixed plate power, CCP $=0 \mathrm{~W}$, $S F_{6}=400$ sccm and $T_{\text {electrode }}=20^{\circ} \mathrm{C}$ for 10 min etching at about $10 \%$ loading. The substrate height (SH), chamber pressure ( $P$ ) and ICP power were varied. 
SiRN is low and pressure build-up of reaction gas inside the channels might lead to cracking of the SiRN membranes during etching.

Non-directional $\mathrm{SF}_{6}$ plasma etching or highly selective $\mathrm{XeF}_{2}$ vapour etching [3.36] offers good alternatives to wet-chemical etching. A parameter study on high-density $\mathrm{SF}_{6}$ plasma etching with zero self-bias was conducted using the Adixen AMS $100 \mathrm{SE}$ with photoresist as mask (Fig. 3.12). An isotropic profile was expected due to the small $2 \mu \mathrm{m}$ etch holes, however a significant anisotropic etch effect was observed.

The etch profiles and surface roughness were observed to be in agreement with results obtained using similar etch parameters on larger mask openings [3.37]. In general, it was observed that etching at a lower pressure results in a more isotropic channel profile.

The best channel profile, with good uniformity across the wafer at high etch speed, was obtained applying an ICP of $2500 \mathrm{~W}$ at $20 \mathrm{~m}$ Torr with a substrate height of $110 \mathrm{~mm}$. A vertical etch speed of $1.5 \mu \mathrm{m} \cdot \mathrm{min}^{-1}$, with a relative lateral etch speed of 0.75 , was measured for microchannels etched with these settings for 10 minutes, with an etch rate of $25 \mathrm{~nm} \cdot \mathrm{min}^{-1}$ measured for SiRN.

\subsection{Surface Microchannel Process Results}

Several wafers were processed with surface microchannels etched using the Adixen AMS $100 \mathrm{SE}$ with the described process for 10 minutes, yielding microchannels with approximately $15 \mu \mathrm{m}$ diameter. Several aspects of the surface channel technology were thereafter investigated and a functional thermal flow sensor was fabricated.

\subsubsection{Release of Device Templates by KOH Etching}

Various device templates were designed, exploring the use of surface microchannels for a range of microfluidic applications. The device templates were released by $\mathrm{KOH}$ etching and fluidic entrance holes were created on the frontside of the wafer. An additional mask was used for the release of the surface microchannels from the backside of the wafer, allowing for a closer inspection of the surface microchannels.

Figure 3.13 shows crossing microchannels observed form the backside of the wafer. The microchannels are emerging from $\{111\}$-planes created by $\mathrm{KOH}$ etching and are merged with the SiRN membrane on the frontside of the wafer. The template demonstrates the possibility in creating microfluidic networks for lab-on-chip applications. A range of designs can be realised, including e.g. reactor chambers, static micromixers and micro heat exchangers. Integration of heaters on top of freelysuspended microchannels allow for the efficient heating of the fluid, while rapid cool- 


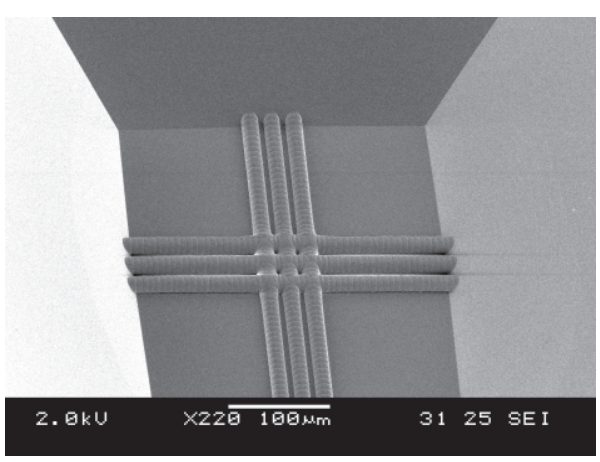

a)

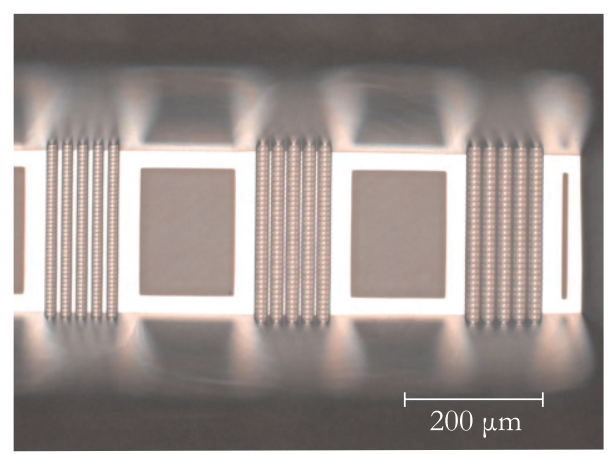

b)

Fig. 3.13 Device template for lab-on-chip applications, with a) crossing microchannels on a SiRN membrane and b) suspended microchannels seen from the backside.

ing to ambient temperature can be achieved by the embedding of microchannels in the bulk silicon of the substrate.

Figure 3.14 shows a freely suspended surface microchannel on the frontside of the wafer. The channel is thermally isolated from the substrate by a cavity created by $\mathrm{KOH}$ etching. The device template has been used in the implementation of a calorimetric thermal flow sensor (Chap. 4).

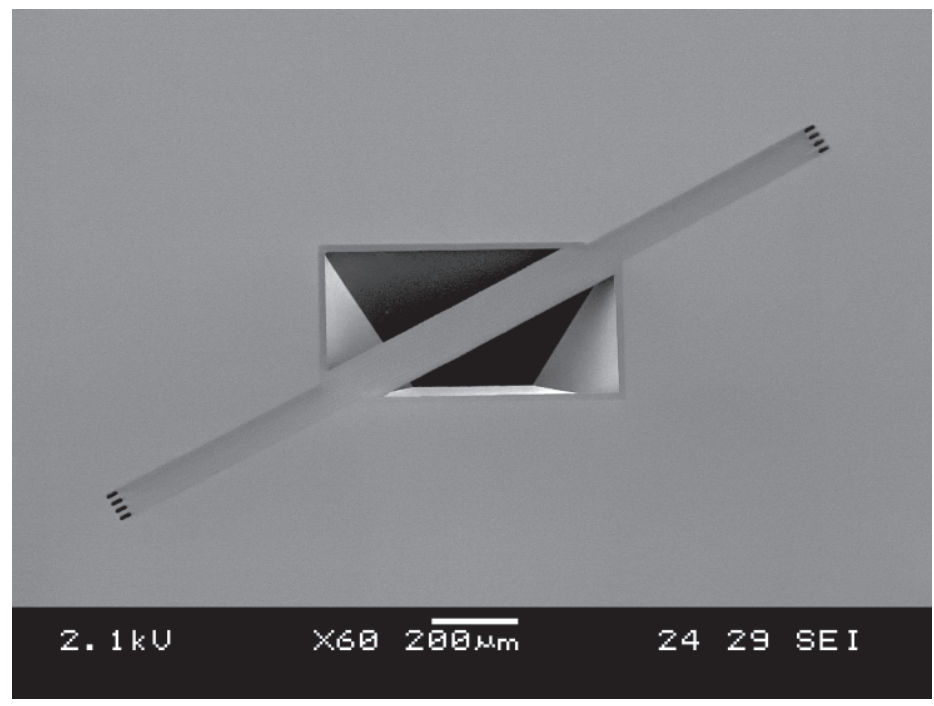

Fig. 3.14 Device template for surface microchannel thermal flow sensor. 


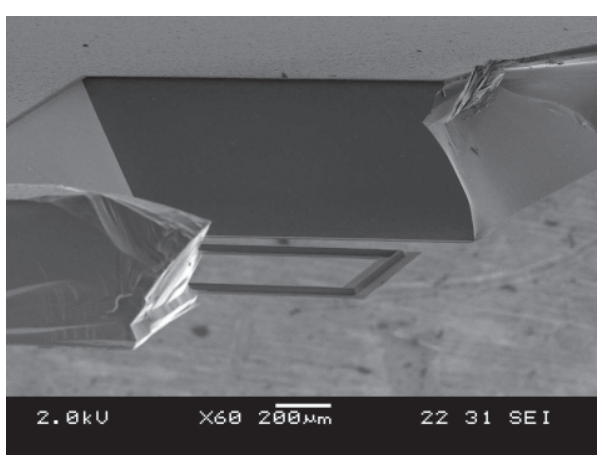

a)

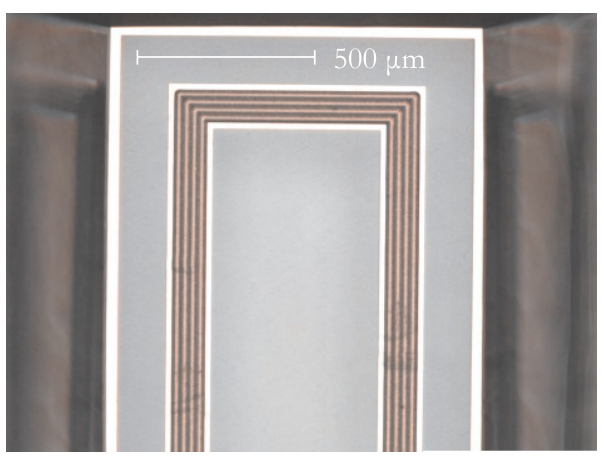

b)

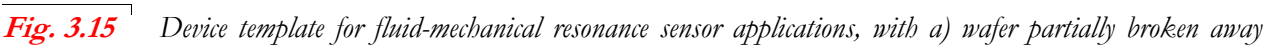
and b) U-shaped resonance structure containing several merged microchannels seen from the backside.

Figure 3.15 shows $1 \mathrm{~mm}$ long surface microchannel resonance structures broken out of the wafer. The freely-suspended surface microchannel is slightly deflected outof-plane, due to the inherent structural strength of the surface microchannel.

The device template can be used in fluid-mechanical resonance applications. Figure 3.16 shows the first resonance mode on a freely suspended microchannel resonance structure measured with a laser-vibrometer using a piezo stack for actuation.

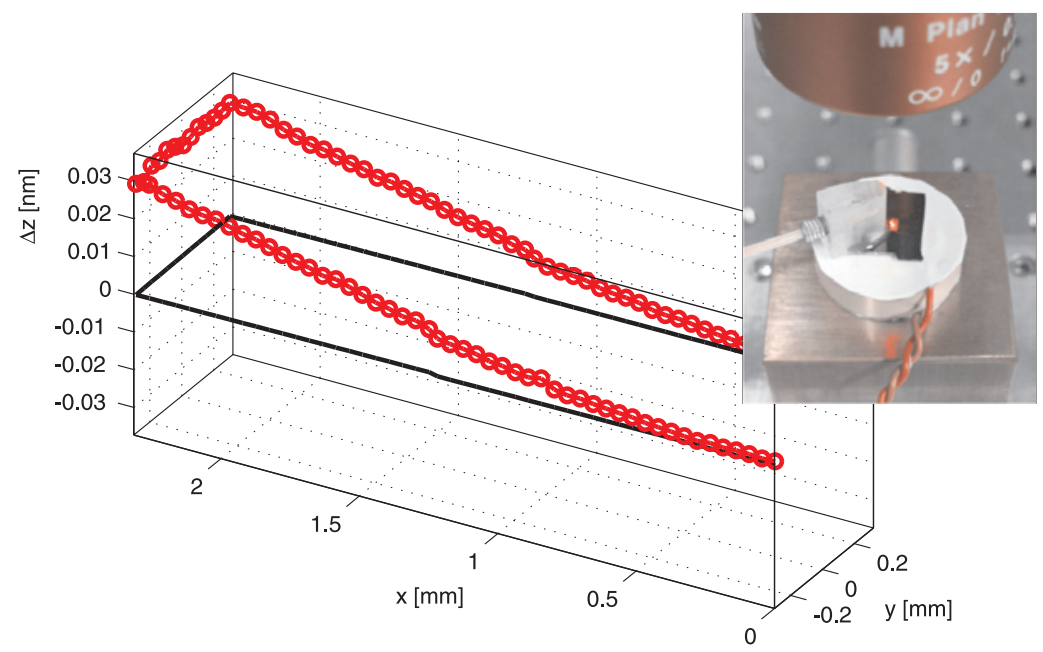

Fig. 3.16 First resonance mode at $6.4 \mathrm{kHz}$ of a fluid-resonance device template filled with air. The inset shows the fluidic connection to the microchannels, the laser-vibrometer objective and a piezo stack for actuation. 


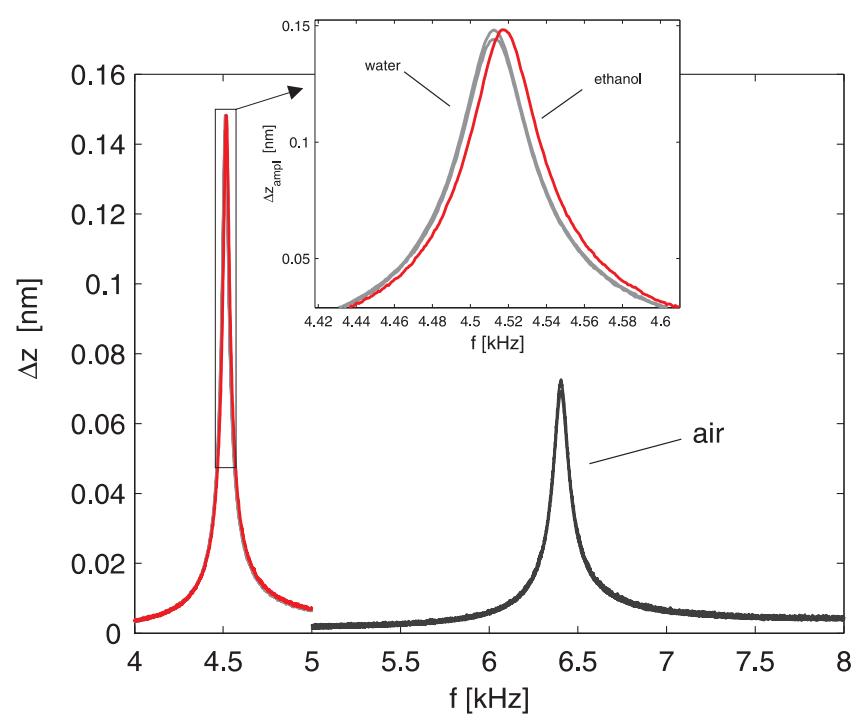

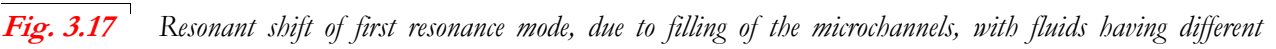
densities.

Figure 3.17 shows the possibility to measure fluid density by measuring the frequency shift of the first resonance mode. Haneveld et al. [3.29] demonstrated that a highly-sensitive Coriolis flow sensor can be obtained by adding comb-like electrodes and applying Lorentz-force actuation.

\subsubsection{Release of Device Templates by $\mathrm{SF}_{6}$ Plasma}

Release of surface microchannels by non-directional $\mathrm{SF}_{6}$ plasma release (App. A.3) was investigated on device templates intended for use in thermal flow-sensor applications, while photoresist should remain on the microchannels for the protection of transducer material. Surface microchannel structures were released by $\mathrm{SF}_{6}$ plasma, applying the same recipe used for the etching of the microchannels and applying a $4 \mu \mathrm{m}$ thick photoresist layer. Figure 3.18a shows a device templates released after 15 min etching, with photoresist still remaining on the freely-suspended microchannel. Large freely-suspended microchannels require $\mathrm{SF}_{6}$ plasma etching at $1.8 \mathrm{~kW} \mathrm{ICP}$ power, $220 \mathrm{~mm} \mathrm{SH}, 350 \mathrm{sccm} \mathrm{SF} 6$ flow and $15 \%$ valve at $0{ }^{\circ} \mathrm{C}$ preventing the heating of the freely-suspended microchannels and burning of the photoresist.

Figure $3.18 \mathrm{a}$ also shows that ridges remain below the released microchannels and electrical contact-lead support structures. The height of these ridges depends on the size of the access windows and width of the freely-suspended structures. 


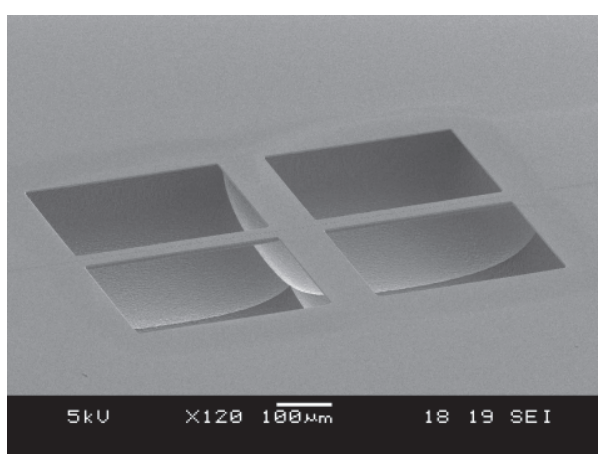

a)

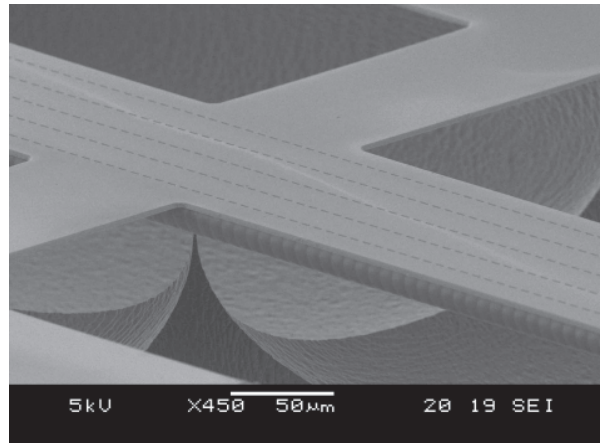

b)

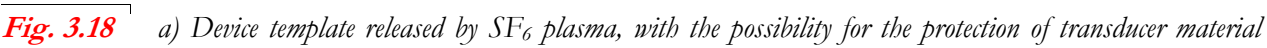
with photoresist. b) Nearly released device, a small spike of Si makes thermal contact with the channels.

Figure $3.18 \mathrm{~b}$ shows a device template also etched for $15 \mathrm{~min}$, which is nearly released. The structure has several merged microchannels, requiring further etching to be released fully. To successfully release surface microchannel devices with $\mathrm{SF}_{6}$ plasma requires sufficiently large access windows to the silicon and sufficiently thick photoresist for the protection of transducer material.

\subsubsection{Packaging of Surface Microchannels Devices}

The possibility for the wafer-level packaging of surface microchannel devices by anodic bonding was investigated. A wafer containing surface microchannels was anodically bonded to a Pyrex wafer, maintaining $450{ }^{\circ} \mathrm{C}$ for $1.5 \mathrm{hrs}$ applying $800 \mathrm{~V}$. Anodic bonding to the SiRN layer is made possible by wet-oxidation of the SiRN layer at $1050{ }^{\circ} \mathrm{C}$ for $1.5 \mathrm{hrs}$ [3.38]. The oxidation has to be done before the integration of thin-film transducers. Wet-oxidation resulted in large stress in the SiRN layer, which can lead to breaking of the microchannel membranes. This was later solved by 1 min deposition of TEOS, which resulted in low stress in the SiRN layer, while anodic bonding to a Pyrex wafer was successful.

The Si wafer was removed by $\mathrm{KOH}$ etching after wet-oxidation and anodic bonding, resulted in surface microchannels on a Pyrex wafer, allowing for the inspection of the bond interface (Fig. 3.19a). Figure 3.19b shows that the SiRN layer surrounding the microchannels is everywhere perfectly bonded to the Pyrex wafer. Inspection from the opposite side revealed that the microchannel membranes were also properly bonded to the Pyrex wafer, confirming that wafer-level packaging and possibly vacuum sealing of surface microchannel devices can be achieved. 


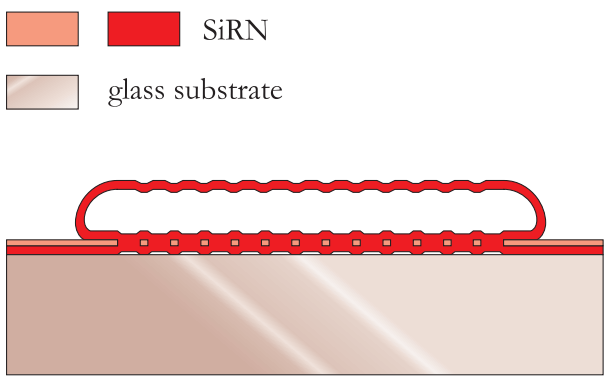

a)

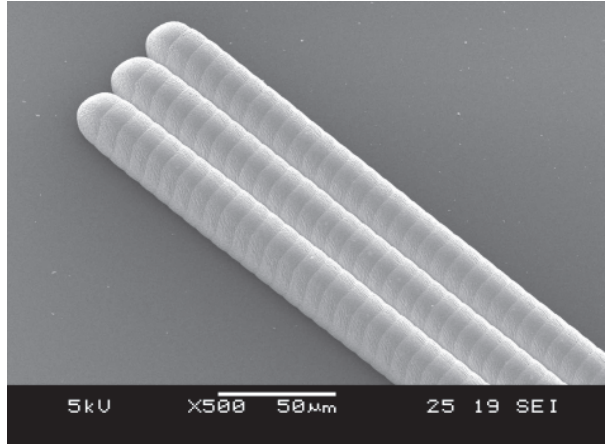

b)

Fig. 3.19 Surface microchannels on a glass substrate, after anodic bonding and removal of the Si wafer by KOH wetetching.

A capping wafer using a $200 \mu \mathrm{m}$ thin Borofloat substrate has been designed, together with Micronit Microfluidics B.V., for the wafer-level packaging of surface microchannel devices. The capping wafer contains $50 \mu \mathrm{m}$ deep wet-etched vacuum chambers (Fig. 3.20a), which continue as far as possible towards the wafer through contact holes (Fig. 3.20b). This is required for fast pump-down time, reaching highvacuum in the Knudsen flow regime. Electrical contact leads on the process are fed through $300 \mathrm{~nm}$ deep nanocavities wet-etched in the capping wafer. Wafer through contact holes are wet-etched from the opposite side of the capping wafer, intentionally giving a sharp edge near the electrical contacts on the device wafer. This is to reduce shadowing effect during evaporation of the metal seal (Fig. 3.11). It should allow for proper sealing of the vacuum chamber under high-vacuum.

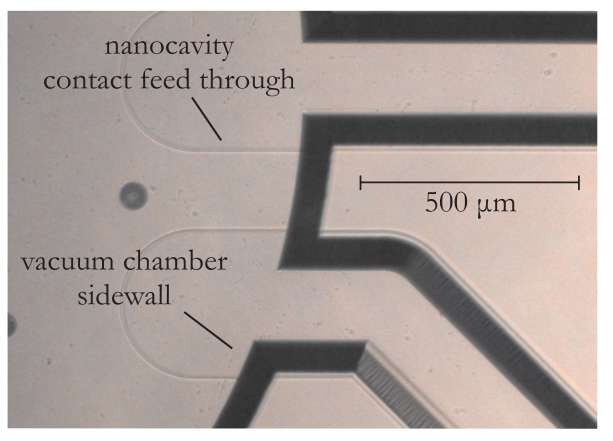

a)

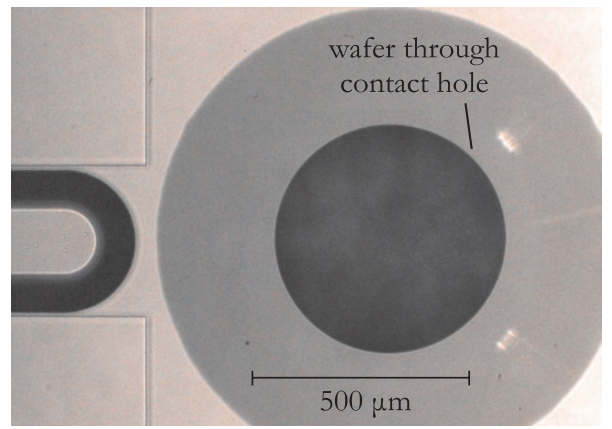

b)

Fig. 3.20 Glass capping wafer for the wafer-level pack aging of surface microchannel devices. 


\subsection{Conclusions}

A versatile concept for the creation of freely suspended surface microchannels using a straightforward and reliable process was demonstrated. Surface channel technology (SCT) allows for easy fluidic interconnection and integration of transducer material in close proximity to the fluid. Complex microfluidic networks can be created, using a single mask defining etch holes. The microchannels were fabricated directly below the substrate surface, leaving a planar substrate, allowing for standard fabrication processes to be readily applied for the integration of transducers. The microchannels were etched using high-density $\mathrm{SF}_{6}$ plasma with zero self-bias, resulting in good uniformity of the microchannels across the wafer. The microchannels were released and fluidic entrance holes were subsequently created. Several device templates have demonstrated the versatility of the concept. Release by non-directional $\mathrm{SF}_{6}$ plasma allows for the protection of transducer structures by photoresist, required for the integration of any required type of transducer material. Additionally, surface microchannel devices can be packaged by anodic bonding using a glass capping wafer.

\section{References}

[3.1] M. Dijkstra, M.J. de Boer, J.W. Berenschot, T.S.J. Lammerink, R.J. Wiegerink, M. Elwenspoek, “A versatile surface channel concept for microfluidic applications", J. Micromech. Microeng., 17 (2007) 1971-1977.

[3.2] M. Dijkstra, M.J. de Boer, J.W. Berenschot, T.S.J. Lammerink, R.J. Wiegerink, M. Elwenspoek, "Miniaturized thermal flow sensor with planar integrated sensor structures on semicircular surface channels", Sensor. Actuat. A-Phys., 143 (2008) 1-6.

[3.3] M. Dijkstra, T.S.J. Lammerink, R.J. Wiegerink, M. Elwenspoek, "Nano-flow thermal sensors applying dynamic $\omega-2 \omega$ sensing method", Proc. MME (2006).

[3.4] Y. Mizuno, M. Liger, Y-C. Tai, "Nanofluidic flowmeter using carbon sensing element", Proc. IEEE MEMS (2004) 322-325.

[3.5] P. Enoksson, G. Stemme, E. Stemme, "A silicon resonant sensor structure for Coriolis mass-flow measurements”, J. Microelectromech. S., 6 (1997) 119-125.

[3.6] P. Enoksson, "Novel resonant micromachined silicon devices for fluid applications", Ph. D. thesis (1997), Royal Institute of Technology, Stockholm, Sweden.

[3.7] T. Corman, P. Enoksson, G. Stemme, "Low-pressure-encapsulated resonant structures with integrated electrodes for electrostatic excitation and capacitive detection”, Sensor. Actuat. A-Phys., 66 (1998) 160-166.

[3.8] T. Corman, "Low pressure encapsulation techniques for silicon resonators", Ph. D. thesis (1998), Royal Institute of Technology, Stockholm, Sweden.

[3.9] M. Najmzadeh, S. Haasl, P. Enoksson, “A silicon straight tube fluid density sensor", J. Microelectromech. S., 17 (2007) 1657-1663.

[3.10] D. Sparks, R. Smith, M. Straayer, J. Cripe, R. Schneider, A. Chimbayo, S. Anasari, N. Najafi, "Measurement of density and chemical concentration using a microfluidic chip", Lab Chip, 3 (2003) 19-21. 
[3.11] S. Tadigadapa, C. Tsai, Y. Zhang, N. Najafi, "Micromachined fluidic apparatus", U.S. patent (1999), Ann Arbor, MI, United States.

[3.12] D. Westberg, O. Paul, G.I. Andersson, H. Baltes, "A CMOS-compatible device for fluid density measurements fabricated by sacrificial aluminium etching, Sensor. Actuat., 73 (1999) 243-251.

[3.13] T. Burg, S.R. Manalis, "Suspended microchannel resonators for biomolecular detection”, Appl. Phys. Lett., 83 (2003) 2698-2700.

[3.14] M. Agah, K.D. Wise, “A fully-dry PECVD-oxynitride process for microGC column fabrication", Proc. IEEE MEMS (2005) 774-777.

[3.15] C-J. Lu, W.H. Steinecker, W-C. Tian, M.C. Oborny, J.M. Nichols, M. Agah, J.A. Potkay, H.K.L. Chan, J. Driscoll, R.D. Sacks, K.D. Wise, S.W. Pang, E.T. Zellers, "First-generation hybrid MEMS gas chromatograph", Lab Chip, $\mathbf{5}$ (2005) 1123-1131.

[3.16] J. Potkay, G.R. Lambertus, R.D. Sacks, K.D. Wise, “A low-power pressure and temperature-programmable $\mu \mathrm{GC}$ column", Proc. Solid-State Sensors, Actuators and Microsystems Workshop (2006) 144-147.

[3.17] J. Chen, K.D. Wise, “A high-resolution silicon monolithic nozzle array for inkjet printing”, Proc. Transducers (1995) 321-324.

[3.18] W. Kaplan, H. Eldersig, C. Vieider, "A novel fabrication method of capillary tubes on quartz for chemical analysis applications”, Proc. IEEE MEMS (1994) 63-68.

[3.19] J.M. Chong, N.C. MacDonal, "Suspended moving channels and channel actuators for microfluidic applications", Proc. ASME MEMS, 66 (1998) 433-438.

[3.20] D. Czaplewski, M. Zalalutdinov, B. Schmidt, H.G. Craighead, "Fabrication of flexible polymer tubes for micro and nanofluidic applications", J. Vac. Sci. Technol. B., 20 (2002) 2459-2465.

[3.21] M.J. de Boer, R.W. Tjerkstra, J.W. Berenschot, H.V. Jansen, G.J. Burger, J.G.E. Gardeniers, M. Elwenspoek, A. van den Berg, "Micromachining of buried microchannels in silicon", J. Microelectromech. S., 9 (2000) 94-103.

[3.22] R.W. Tjerkstra, M. de Boer, E. Berenschot, J.G.E. Gardeniers, A. van den Berg, M.C. Elwenspoek, "Etching technology for chromatography microchannels", Electrochim. Acta, 42 (1997) 3399-3406.

[3.23] C. Rusu, R. van't Oever, M.J. de Boer, H.V. Jansen, J.W. Berenschot, M.L. Bennink, J.S. Kanger, B.G. de Grooth, M. Elwenspoek, J. Greve, J. Brugger, A. van den Berg, "Direct integration of micromachined pipetttes in a flow channel for single DNA molecule study by optical tweezers", J. Microelectromech. S., 42 (1997) 238-245.

[3.24] P-J. Chen, C-Y. Shin, Y-C. Tai, "Design, fabrication and characterization of monolithic embedded parylene microchannels in silicon substrate", Lab Chip, 6 (2006) 803-810.

[3.25] P-J. Chen, Y-C. Tai, "A micromechanical parylene spiral-tube sensor and its applications of unpowered environmental pressure/temperature sensing”, Proc. IEEE Sensors (2007) 1476-1479.

[3.26] P-J. Chen, D-C. Rodger, R. Agrawal, S. Saati, E. Meng, R. Varma, M.S Humayun, Y-C. Tai, "Implantable micromechanical parylene-based pressure sensors for unpowered intraocular pressure sensing”, J. Micromech. Microeng., 17 (2007) 1931-1938. 
[3.27] L. Giacchino, Y-C. Tai, "Parylene-membrane piezoresistive pressure sensors with $\mathrm{XeF}_{2}$-etched cavity", Proc. IEEE Sensors (2008) 1568-1571.

[3.28] J.G.E. Gardeniers, H.A.C. Tilmans, C.C.G. Visser, "LPCVD silicon-rich silicon nitride films for applications in micromechanics, studies with statistical experimental design", J. Vac. Sci. Technol. A., 14 (1996) 2879-2892.

[3.29] J. Haneveld, T.S.J. Lammerink, M.J. de Boer, R.J. Wiegerink, "Micro Coriolis mass flow sensor with integrated capacitive readout", Proc. IEEE MEMS (2009).

[3.30] O. Brand, Y.B. Gianchandani, O. Tabata, H. Zappe, "Comprehensive microsystems", "Packaging", Elsevier, (2008) chapter 1.15.

[3.31] T.P. Burg, A.R. Mirza, N. Milovic, C.H. Tsau, G.A. Popescu, J.S. Foster, S. Manalis, "Vacuum-packaged suspended microchannel resonant mass sensor for biomolecular detection", J. Microelectromech. S., 15 (2006) 1466-1476.

[3.32] H.A.C. Tilmans, "Micro-mechanical sensors using encapsulated built-in resonant strain gauges", Ph. D. thesis (1993), University of Twente, Enschede, The Netherlands.

[3.33] M. Bartek, J.A. Foerster, R.F. Wolffenbuttel, "Vacuum sealing of microcavities using metal evaporation", Sensor. Actuat. A-Phys., 61 (1997) 364-368.

[3.34] N. Najafi, Y. Zhang, "Electrical feedthrough structures for micromachined devices and methods of fabricating the same", U.S. patent (2002), Ann Arbor, MI, United States.

[3.35] T.T. Veenstra, J.W. Berenschot, J.G.E. Gardeniers, R.G.P. Sanders, M.C. Elwenspoek, A. van den Berg, "Use of selective anodic bonding to create micropump chambers with virtually no dead volume", J. Electrochem. Soc., 148 (2001) G68-G72.

[3.36] H.F. Winters, J.W. Coburn, "The etching of silicon with $\mathrm{XeF}_{2}$ vapor", Appl. Phys. Lett., 34 (1979) 70-73.

[3.37] K.P. Larsen, D.H. Petersen, O. Hansen, "Study of the roughness in a photoresist masked, isotropic, $\mathrm{SF}_{6}$ based ICP silicon etch", J. Electrochem. Soc., 153 (2006) 1051-1058.

[3.38] S. Weichel, R. de Reus, S. Bouaidat, P.A. Rasmussen, O. Hansen, K. Birkelund, H. Dirac, "Low-temperature anodic bonding to silicon nitride", Sensor. Actuat. A-Phys., 82 (2000) 249-253. 


\section{Miniaturised Calorimetric Flow Sensor}

A calorimetric miniaturised flow sensor was realised with a linear sensor response measured for water flow up to flow rates in the order of $300 \mathrm{nl} \cdot \mathrm{min}^{-1}$. The surface channel technology has been applied in the realisation of a sensor with a thermally isolated freely-suspended silicon-rich silicon-nitride microchannel directly below the substrate surface. The microchannel fabrication concept allowed for the planar integration of sensor structures in close proximity to the fluid, while chemical-resistant fluidic connections were made directly on top of the microchannel, without introducing large dead volumes. The realised flow sensor consists of a microchannel with low bydraulic resistance and $4.5 \mathrm{nl}$ total fluid volume. A pressure driven flow setup was used to force water through the microchannel, measuring output sensitivity in the order of $0.2 \mu \mathrm{V} / \mathrm{nl}^{\mathrm{min}} \mathrm{m}^{-1}$ for flows up to about $300 \mathrm{nl} \cdot \mathrm{min}^{-1}$. The measured sensor output is in close agreement with results obtained from both a detailed and an approximate numerical model of the sensor. ${ }^{4.1}$

\subsection{Introduction}

Fluidic applications within the field of micro- and nanofluidics often require the accurate measurement of small fluid flows, in the order of $\mathrm{nl} \cdot \mathrm{min}^{-1}$. For such small flow rates, standard bulk micromachined flow channels are not suitable, because a large part of the flow is in thermal contact with the substrate and does not contribute to the sensor signal. Instead, thermally isolated channels are required. This chapter describes a miniaturised flow sensor based on freely suspended silicon-rich siliconnitride semicircular microchannels, which are thermally isolated from the substrate. The hydraulic resistance of the microchannels can be freely chosen by adjusting the microchannel diameter. This gives advantage over freely-suspended surface micromachined channels [4.2-4.4], which have large hydraulic resistance of the microchannel, mostly determined by the channel height being limited to only a few $\mu \mathrm{m}$. Additionally, surface micromachined channel can give step-coverage problems for sensor structures required to be deposited across the microchannel, whereas the flow sensor described in this chapter is readily made below the substrate surface, allowing for the planar integration of sensor structures.

\footnotetext{
4.1 This chapter is based on an article published in Sensor. Actuat. A-Phys. [4.1].
} 


\subsection{Sensor Design}

Figure 4.1 illustrates the design of the miniaturised calorimetric flow sensor. The sensor measures liquid flow through a freestanding microchannel crossing a thermalisolation cavity over a length of $800 \mu \mathrm{m}$. Fluidic interfacing to the microchannel is made possible by directly applying O-rings on top of the microchannel inlet and outlet embedded in the substrate surface. The $5 \mathrm{~mm}$ long microchannel is approximately $15 \mu \mathrm{m}$ deep and $80 \mu \mathrm{m}$ wide, having $4.5 \mathrm{nl}$ total volume and a hydraulic resistance as low as $6.17 \mathrm{kPa} / \mu \mathrm{l} \cdot \mathrm{min}^{-1}$ for water.

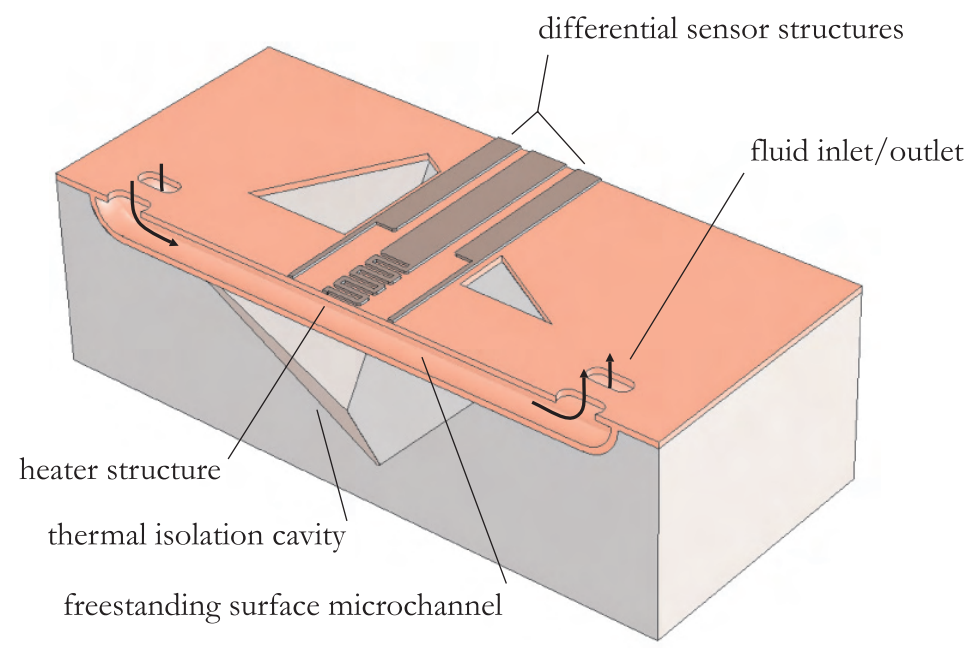

Fig. 4.1 Cross-section of calorimetric flow sensor design, with heater structure and differential sensor structures integrated on a freestanding surface microchannel

Fluid flow is measured by heating the fluid using a heater centered on the suspended microchannel. Heat conduction through the surrounding air, the microchannel and the electrical connection leads results in a symmetric temperature profile around the heater. The temperature profile is disturbed by forced convection on applying fluid flow through the microchannel. The disturbance does not affect the temperature of the heater for small flows, because there is no temperature gradient at the heater for the undisturbed symmetric temperature profile, required for the advection of heat.

The temperature disturbance is measured differentially by measuring the resistance change of two resistors located $40 \mu \mathrm{m}$ up- and downstream from the heater. The sensor output is linearly proportional to the flow rate for small flows, resulting in a highly-sensitive calorimetric flow sensor. The differential sensor output is insensitive to common-mode ambient temperature fluctuations and variations in fluid tempera- 
ture [4.5]. The disturbed temperature profile upon increased fluid flow causes a gradient in temperature across the heater, leading to heat-loss at the heater, eventually decreasing the heater temperature, with the sensor output becoming non-linear.

\subsection{Sensor Fabrication}

The fabrication of the miniaturised calorimetric flow sensor is based on surface channel technology [4.6] as explained in Chap. 3. Surface channel technology is a good alternative, compared to surface micromachined channels, in realising mechanically strong freely suspended microchannels, without the requirement for a sacrificial layer. This allows for the integration of sensor structures in close proximity to the fluid, with improved fluidic interfacing to the microchannels.

Surface microchannels were created by isotropic dry etching, using high-density $\mathrm{SF}_{6}$ plasma with zero self-bias, through small etch holes in a low-stress $500 \mathrm{~nm}$ SiRN (silicon-rich silicon nitride) layer. The coalescence of the etched surfaces resulted in semicircular channel profiles (Fig. 4.2a). Four parallel microchannels were merged into a single microchannel. This reduces the hydraulic resistance of the microchannel to $6.17 \mathrm{kPa} / \mu \mathrm{l} \cdot \mathrm{min}^{-1}$ for water and the microchannel membrane gives a sufficiently large platform for the integration of sensor structures.

The small etch holes and the inner surfaces of the microchannels are uniformly coated by the LPCVD deposition of a second low stress $1.3 \mu \mathrm{m}$ SiRN layer (Fig 4.2b), thus creating completely sealed microchannels, while leaving a planar substrate surface

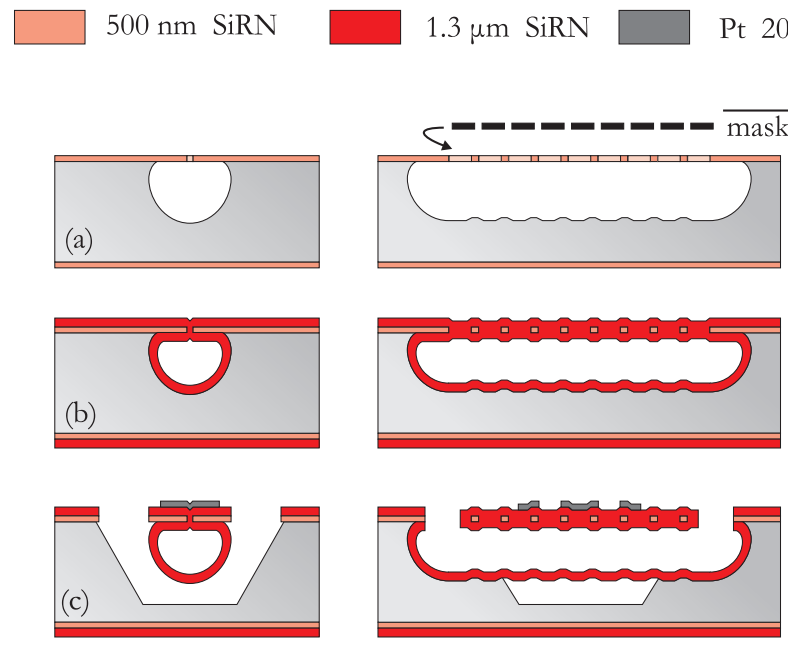

Fig. 4.2 Process scheme for the fabrication of the miniaturised calorimetric flow sensor using Pt sensor structures. 
for further processing (Fig. 4.3). The semicircular channels are mechanical strong and are insensitive to deformation, possibly caused by the integration of sensor materials with high stress across the microchannel. Because of the resulting planar substrate surface, step-coverage problems are avoided and $200 \mathrm{~nm}$ thin-film Pt sensor structures could be deposited directly on top of the microchannels (Fig. 4.2c). The SiRN microchannel membrane was roughened with an $\mathrm{SF}_{6}-\mathrm{O}_{2}$ plasma for proper adhesion of the Pt without the need for an adhesion layer. The Pt was patterned by oxidation using $\mathrm{O}_{2}$ plasma, with the thin oxidation layer acting as a mask during Aqua Regia etching [4.7], creating perfectly defined sensor structures (Fig. 4.4b).

Fluid entrance holes were etched through the SiRN layer (Fig. 4.2c), allowing for the direct interfacing to the microchannels on the frontside of the wafer. Thermalisolation cavities are defined using the same mask (Fig. 4.2c) and are etched by $\mathrm{KOH}$ etchant. The microchannel and electrical contact leads are offset at a $30^{\circ}$ angle to the $<100>$-direction, such that the Si $\{111\}$-planes can be etched underneath the freelysuspended structure, suspending the surface microchannels above the heat conducting substrate.

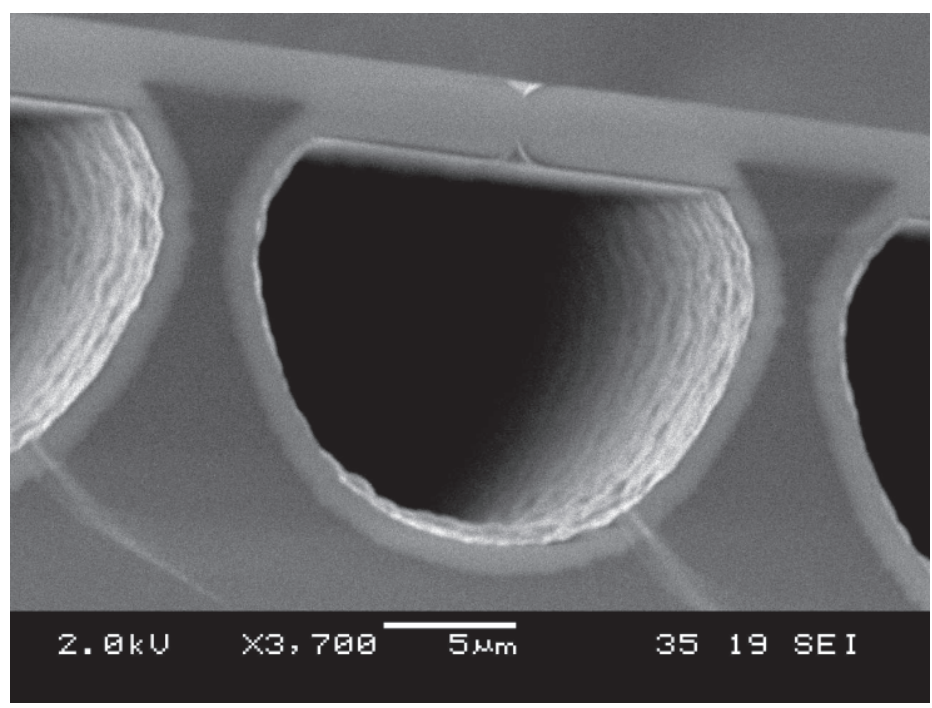

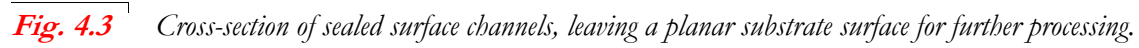




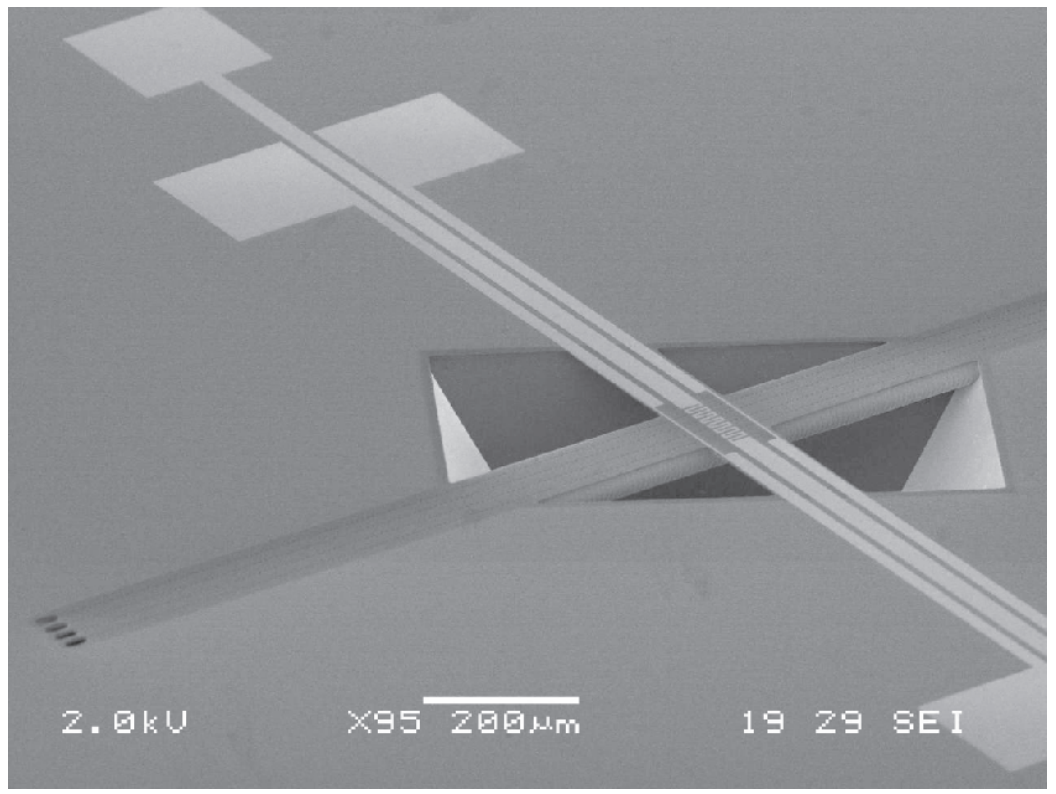

a)

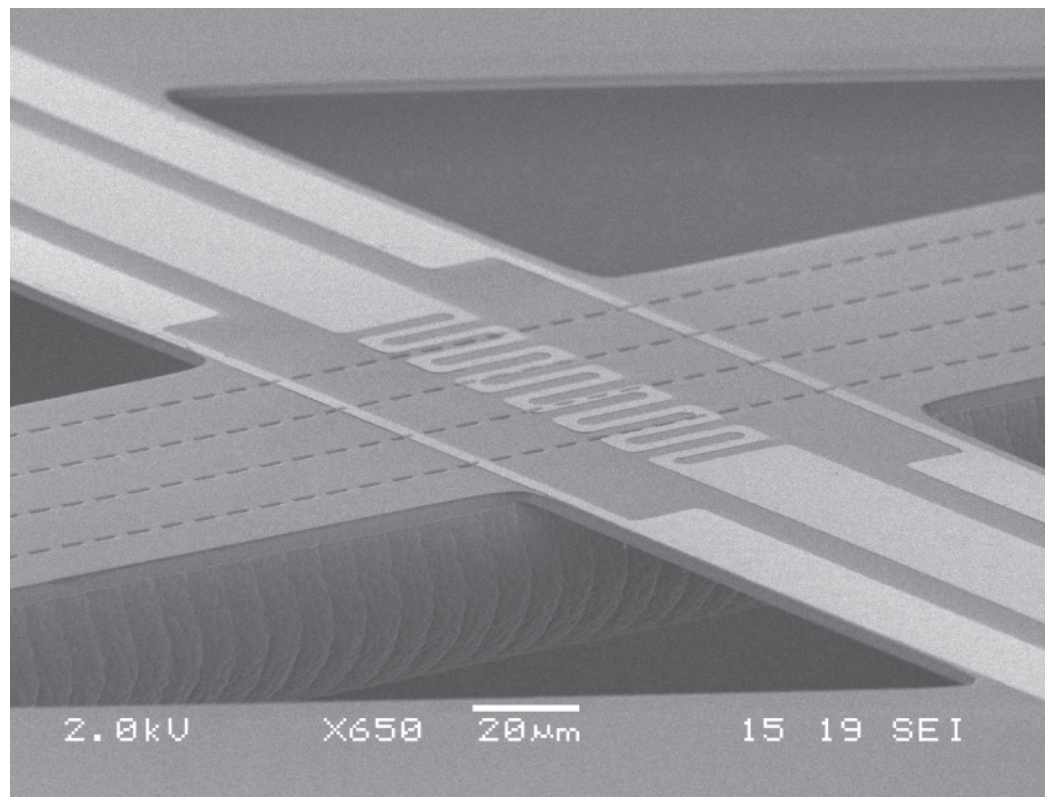

b)

Fig. 4.4 Micrographs of a) calorimetric flow sensor suspended above thermal-isolation cavity and b) Pt resistors crossing surface microchannels. 


\subsection{Sensor Modelling}

\subsubsection{Fluidic-Thermal CFDRC Model}

The temperature field surrounding the heater was solved with CFDRC [4.8] using a high-fidelity mesh of the sensor. The thermal field was solved numerically for the freely-suspended microchannel containing water, the electrical contact leads, the substrate and the surrounding air in the isolation cavity as well as part of the air above the sensor. Material properties where defined according to values in Tbl.4.1. Boundary conditions were set to reside at ambient temperature. Uniform heating power-densities, determined by sheet-resistance and mask-layout data, were imposed on the heater and on the freely-suspended heater connection leads, with the heater dissipating $1.9 \mathrm{~mW}$. A laminar flow profile was solved inside the microchannel for various water flow rates, visualising the disturbance on the temperature field by forced convection due to the fluid flowing through the surface microchannels.

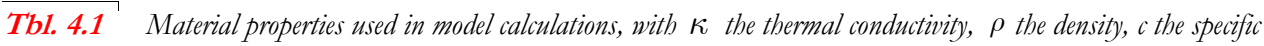
beat and $\mu$ the dynamic viscosity.

\begin{tabular}{cr}
\hline$\kappa_{S i R N}$ & $3 \mathrm{~W} \cdot \mathrm{m}^{-1} \cdot \mathrm{K}^{-1}$ \\
$\kappa_{P t}$ & $72 \mathrm{~W} \cdot \mathrm{m}^{-1} \cdot \mathrm{K}^{-1}$ \\
$\kappa_{S i}$ & $130 \mathrm{~W} \cdot \mathrm{m}^{-1} \cdot \mathrm{K}^{-1}$ \\
$\kappa_{\text {air }}$ & $0.024 \mathrm{~W} \cdot \mathrm{m}^{-1} \cdot \mathrm{K}^{-1}$ \\
\hline
\end{tabular}

\begin{tabular}{cc}
\hline$\kappa_{\text {water }}$ & $0.6 \mathrm{~W} \cdot \mathrm{m}^{-1} \cdot \mathrm{K}^{-1}$ \\
$\rho_{\text {water }}$ & $998 \mathrm{~kg} \cdot \mathrm{m}^{-3}$ \\
$c_{\text {water }}$ & $4179 \mathrm{~J} \cdot \mathrm{kg}^{-1} \cdot \mathrm{K}^{-1}$ \\
$\mu_{\text {water }}$ & $1.12 \mathrm{~N} \cdot \mathrm{s} \cdot \mathrm{m}^{-2}$ \\
\hline
\end{tabular}

Figure 4.5 visualises the calculated asymmetric temperature field on the freelysuspended microchannel due to various water flow rates. It clearly shows that the temperature field extends into the surrounding air due to heat conduction. The temperature isosurface at $0 \mu \mathrm{l} \cdot \mathrm{min}^{-1}$ flow (Fig. 4.5a) is almost spherical. This means that the difference in heat flux through the microchannel and through the surrounding air is proportional to the differences in thermal conductivity. The heat loss to the surrounding air is however significant, because of the increase in surface area of air at larger distances from the heater, whereas the surface area of the microchannel remains constant. This is clear from the isosurface being more ellipsoidal near the heater and rapidly becoming spherical at larger distances from the heater.

The temperature field becomes asymmetric by forced convection on applying fluid flow through the microchannel. At $0.5 \mu \mathrm{l} \cdot \mathrm{min}^{-1}$ water flow rate the fluid inside the microchannel is fully heated to a radial-uniform temperature profile at small axial distances from the heater (Fig. 4.6a). The forced convection is thus dependent on the 


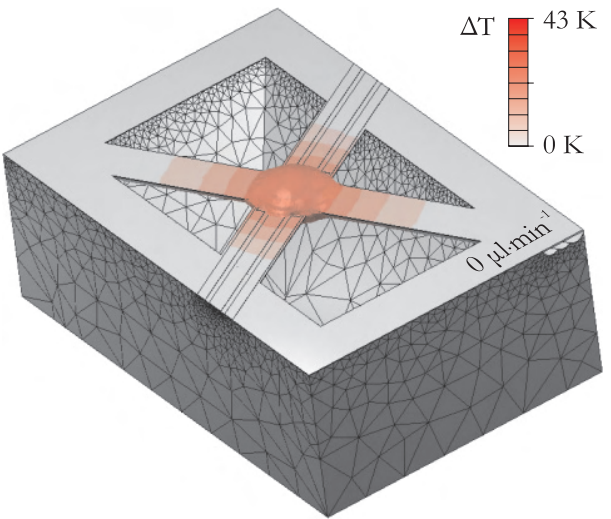

a)

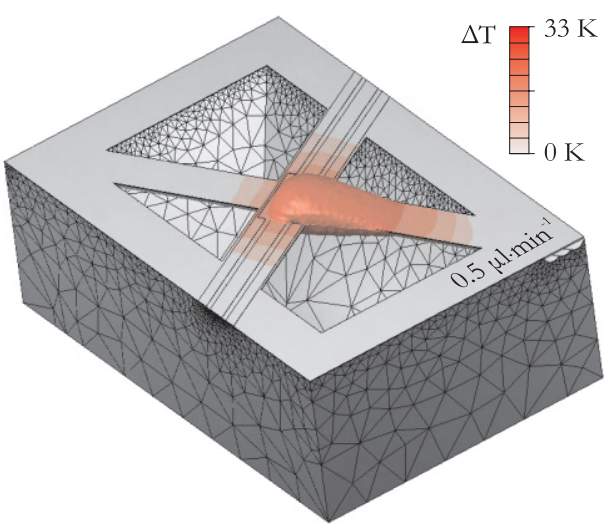

c)

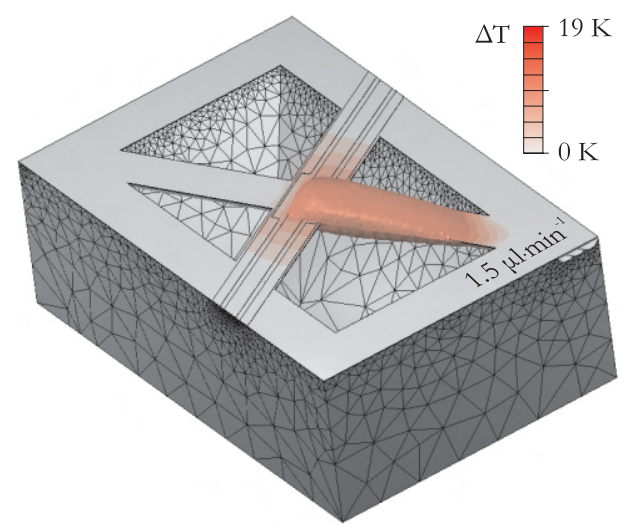

e)

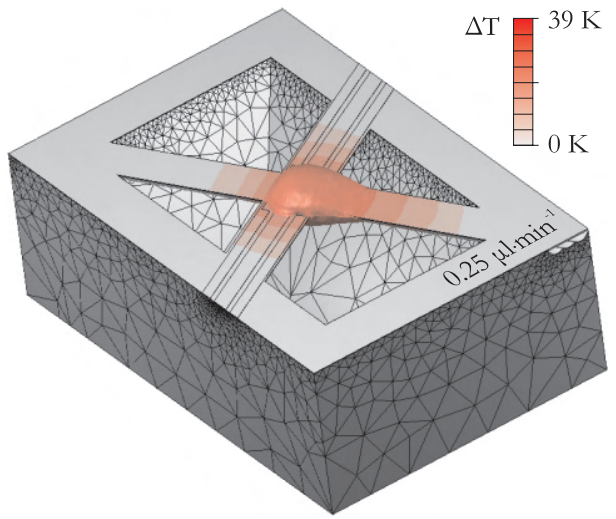

b)

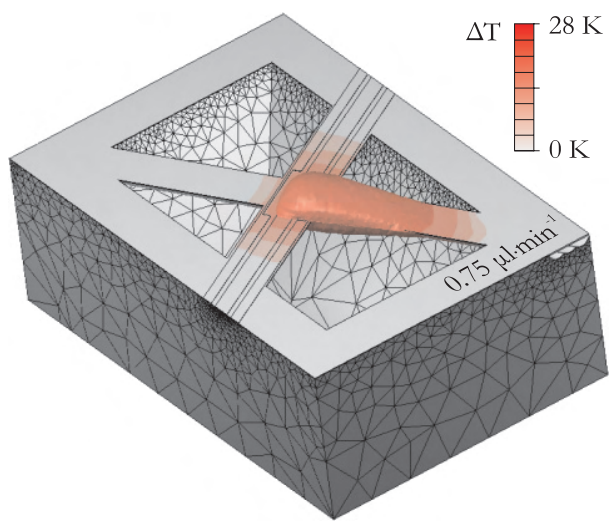

d)

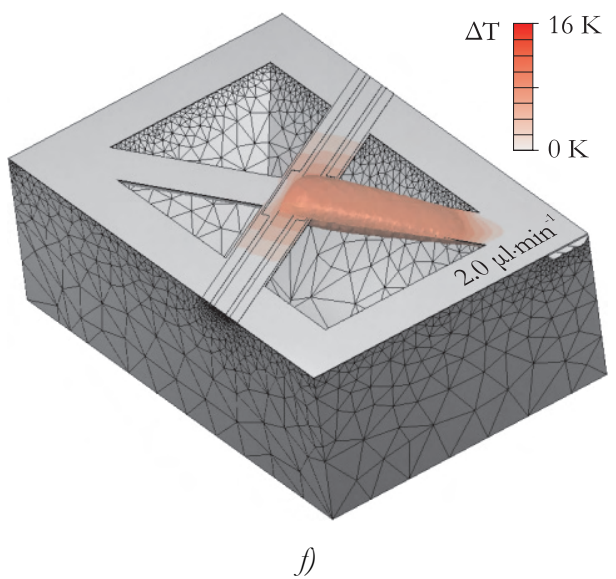

Fig. 4.5 CFDRC model results, showing asymmetric thermal profiles on the thermal flow sensor at various water flow rates applying $1.9 \mathrm{~mW}$ heating power. Isosurfaces at half-maximum temperature are shown. 


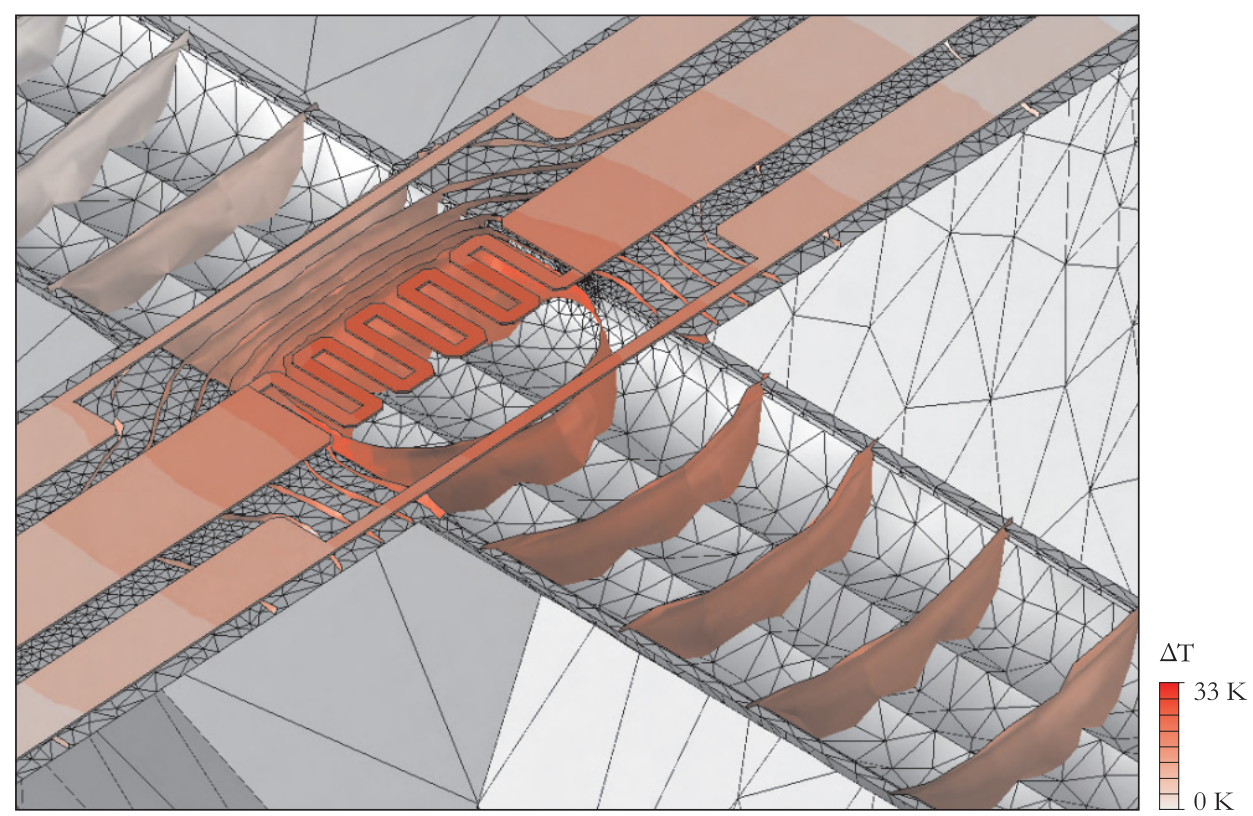

a)

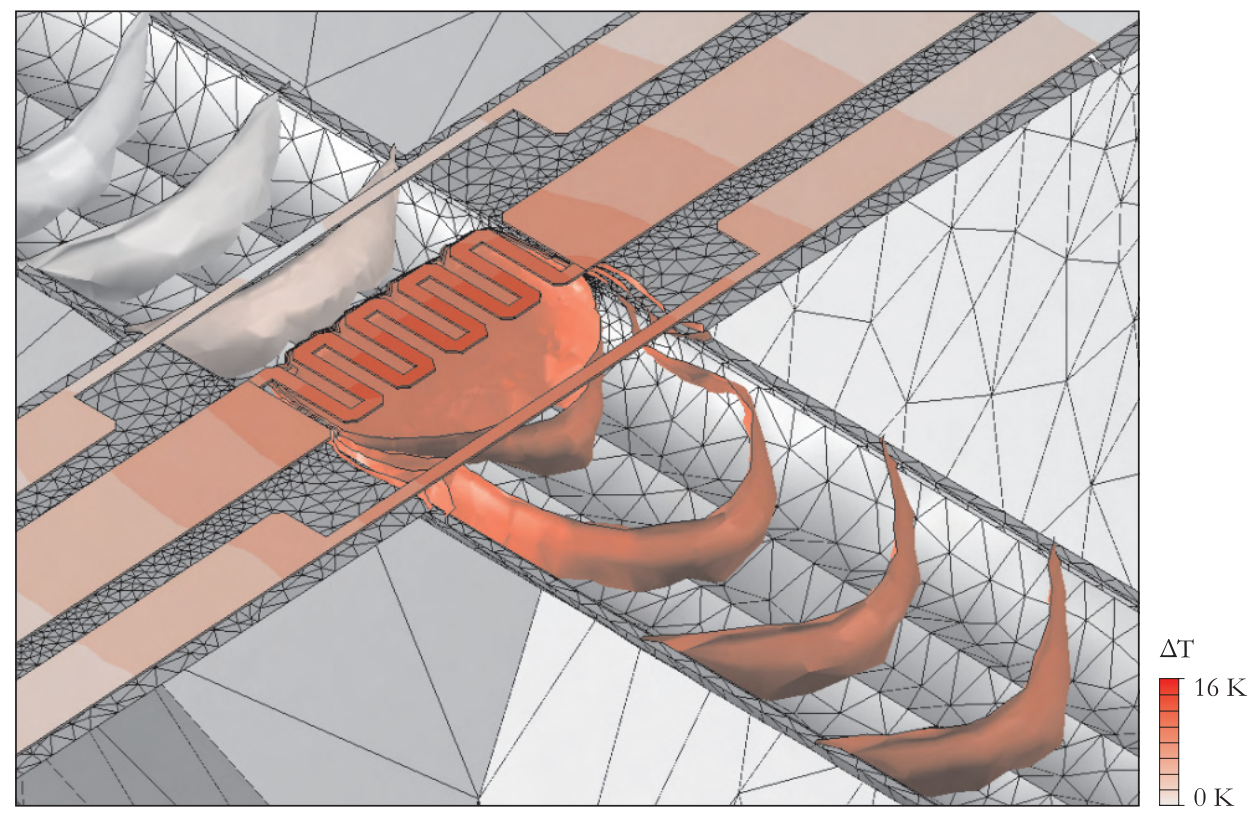

b)

Fig. 4.6 The temperature field inside the freely-suspended microchannel visualised by isosurfaces at $1.9 \mathrm{~mW}$ heating power at a) $0.5 \mu \mathrm{l} \cdot \mathrm{min}^{-1}$ water flow and b) $2 \mu \mathrm{l} \cdot \mathrm{min}^{-1}$ water flow, showing a visible thermal boundary layer. 
total volume flow through the microchannel (Sec. 1.3.2). At $2 \mu \mathrm{l} \cdot \mathrm{min}^{-1}$ water flow a thermal boundary layer extends from the heater, but reaches the microchannel depth before reaching the downstream temperature sensor (Fig. 4.6b). The downstream sensor at $2 \mu \mathrm{l} \cdot \mathrm{min}^{-1}$ water flow measures a temperature, which is not determined by
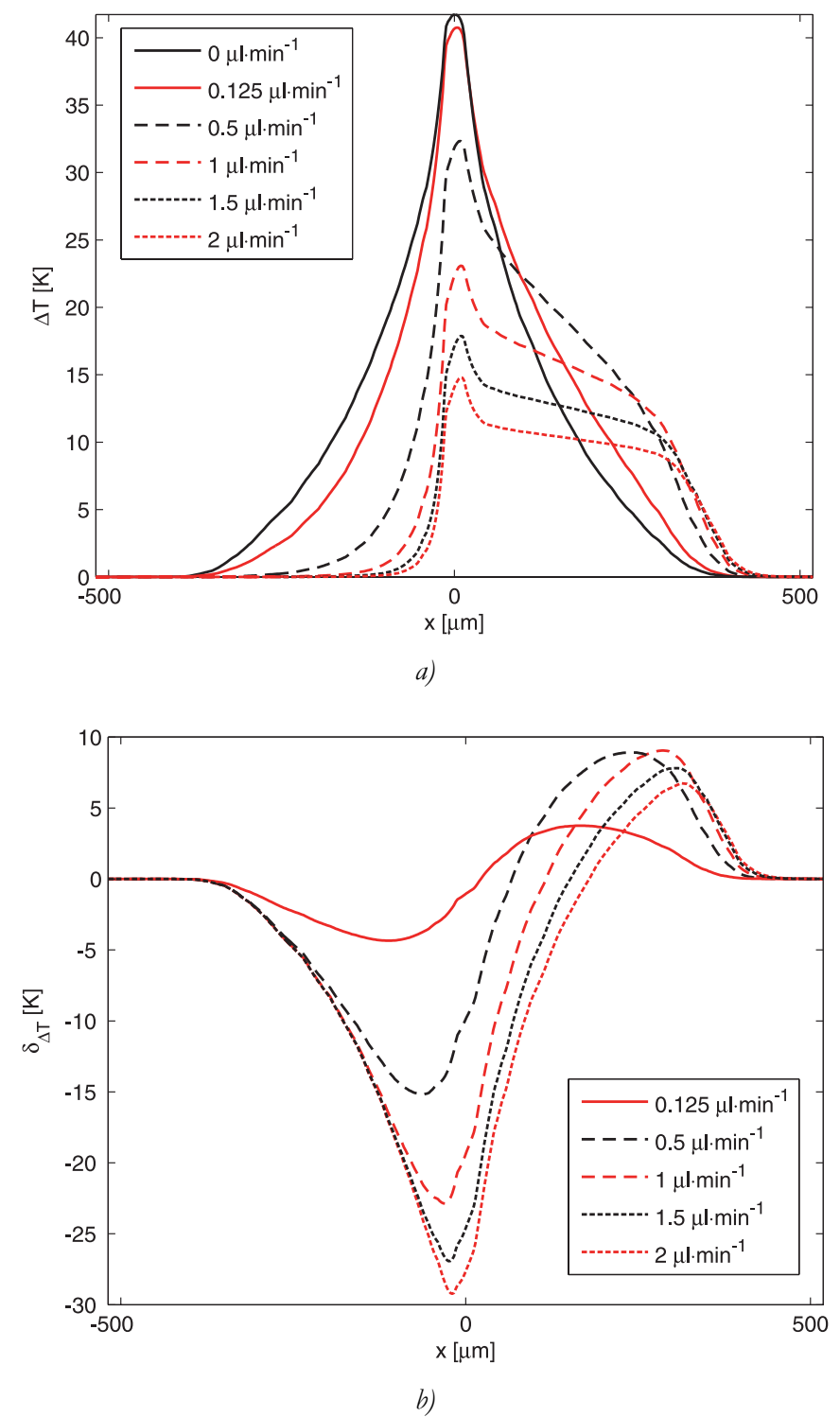

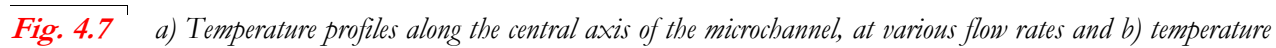
changes relative to the $0 \mu l \cdot \mathrm{min}^{-1}$ temperature profile due to various flow rates. The microchannel is fixed to the substrate at $\pm 400 \mu \mathrm{m}$. 
the thermal boundary layer. Instead, the downstream sensor measures the average of the thermal profile along the width of the microchannel, visible from the large density of isosurfaces near the edge of the microchannel at the downstream sensor and heater. This indicates a relatively large heat loss to the heater and sensor contact leads at $2 \mu \mathrm{l} \cdot \mathrm{min}^{-1}$ water flow rate. A better solution is to have the contact leads positioned along the microchannel (Chap. 5).

The numerical model was solved for various water flow rates from $0 \mu \mathrm{l} \cdot \mathrm{min}^{-1}$ up to $2 \mu \mathrm{l} \cdot \mathrm{min}^{-1}$. Figure 4.7 shows the resulting temperature profiles and the temperature changes by heat convection on the freely-suspended surface microchannel, with the heater centred at $0 \mu \mathrm{m}$. As expected, the heater temperature for small flows is hardly affected and the disturbance by the flow increases the temperature downstream from the heater as much as it decreases the temperature upstream. The numerical results in Fig 4.7b indicate that the optimal position of the temperature sensors is halve way between the heater and the end of the suspended part of the microchannel. The temperature profiles decrease with decreasing heater temperature at higher flow rates, with a rapid decrease in temperature between $300 \mu \mathrm{m}$ and $400 \mu \mathrm{m}$, because there the microchannel is in close proximity to the silicon substrate.

\subsubsection{Thermal FVM Model}

The CFDRC model shows that the temperature field along the microchannel is nearly symmetric around the central axis, where the magnitude of the thermal gradient from the microchannel to the surrounding air is largely being determined by the shortest radial distance to the silicon substrate. The spherical shape of the temperature

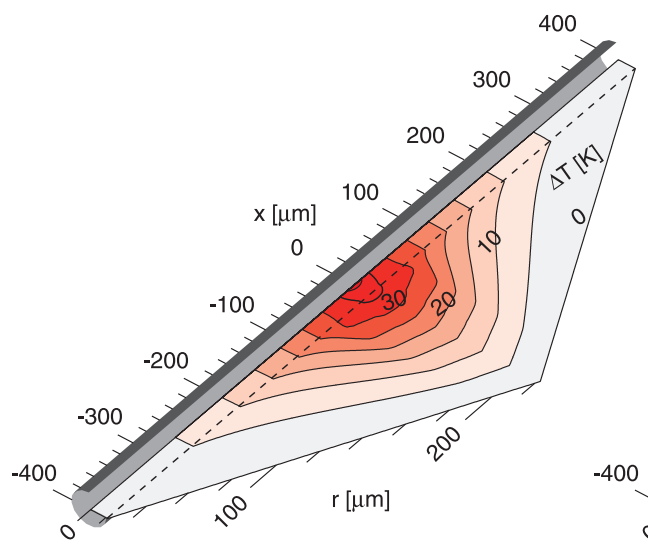

a)

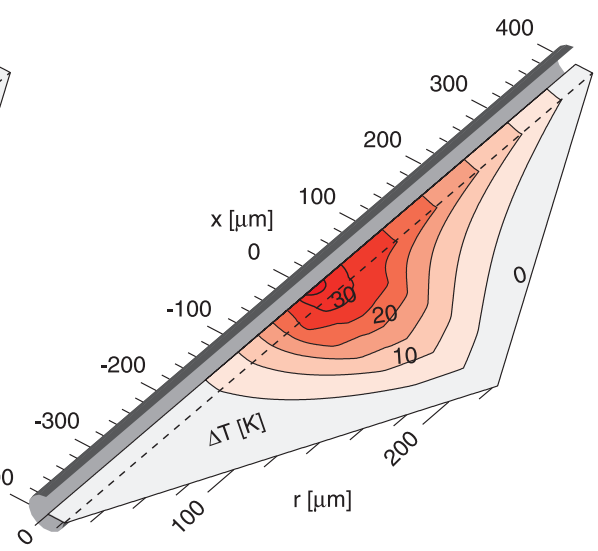

b)

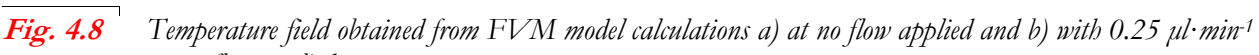
water flow applied. 
isosurface in Fig. 4.5a indicates that heat fluxes in both $x$-direction and $r$-direction have to be considered. These conditions allow for a fast two-dimensional cylindricalsymmetric model of the sensor giving accurate quantitative results. Such a thermal model was constructed in MATLAB [4.9], applying the finite volume method [4.10]. Appropriate boundary conditions and material parameters (Tbl. 4.1) were defined.

Figure 4.8 shows the temperature field resulting from FVM model calculations, with no flow and $0.25 \mu \mathrm{l} \cdot \mathrm{min}^{-1}$ water flow applied at $1.9 \mathrm{~mW}$ heating power. The mesh boundary in $r$-direction is approximated by taking the shortest radial distance to the thermal isolation cavity at each $x$-position along the microchannel. The contact leads are modelled by an approximated thermal conductivity function $\kappa_{\text {lead }}$ dependent on $r$.

Figure 4.9 shows the temperature profiles from FVM model calculations. The FVM model gives equivalent results to CFDRC for flow rates up to $500 \mathrm{nl} \cdot \mathrm{min}^{-1}$. The temperature profiles at higher flow rates differ slightly from CFDRC results shown in Fig. 4.7a, especially near the microchannel suspension due to the close proximity to the silicon substrate. The profiles near the heater are also slightly different from CFDRC results, showing the approximation made by the thermal conductivity function $\kappa_{\text {lead }}$ for the contact leads. The FVM model overall gives fast approximate solutions to the temperature field, giving sufficiently accurate quantitative results.

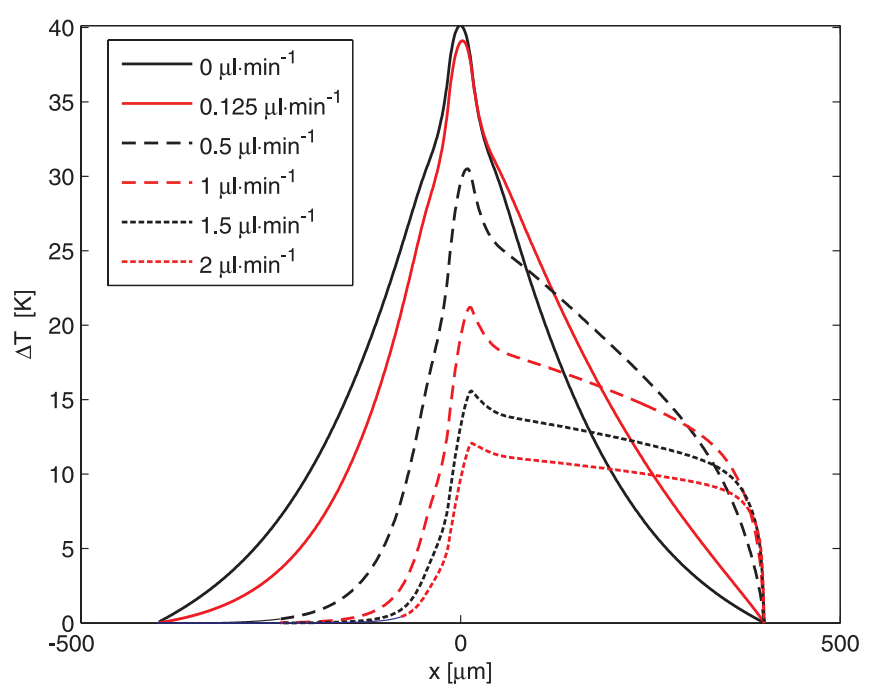

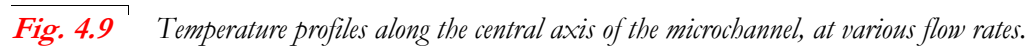




\subsection{Experimental Setup}

Measurements were performed using a pressure driven flow setup as indicated in Fig. 4.10. Water was forced through the microchannel by applying pressure from a $\mathrm{N}_{2}$ gas cylinder. The flow rate was determined for each measurement point by measuring the advancing meniscus in the fluidic connection tube, which contains approximately $0.5 \mu \mathrm{l} \cdot \mathrm{mm}^{-1}$, after applying a constant pressure for several minutes. The flow was kept constant by regulating a membrane valve to a fixed pressure in the range from 0 to 1 bar.

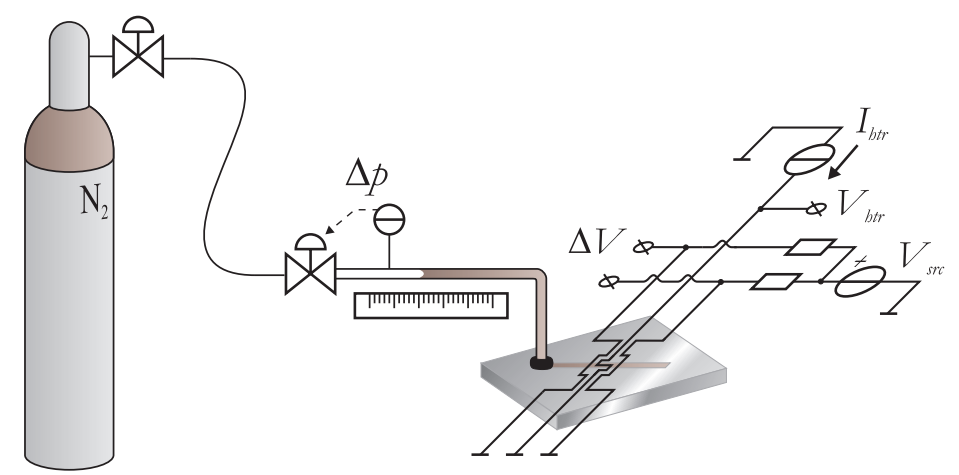

Fig. 4.10 Schematic of experimental setup. Water flow is forced through the sensor chip by a regulated pressure $\Delta p$
from a $N_{2}$ gas cylinder. The heater resistor is heated by a constant current $I_{\text {btr }}$ and the sensor output $\Delta V$ is
obtained using a Wheatstone bridge.

Water flow was measured by applying a current $I_{b t r}$ of $5 \mathrm{~mA}$ to the heater resistor dissipating $1.9 \mathrm{~mW}$. The resistance change of the heater upon fluid flow is measured and can be related to the temperature change of the heater according to $\Delta R=\alpha \cdot \Delta T \cdot R_{0}$, with $\alpha$ the temperature coefficient of resistivity and $R_{0}$ the resistance value at ambient temperature. Van der Pauw structures were calibrated against a PT100 temperature sensor applying a temperature sweep from $20^{\circ} \mathrm{C}$ up to $120^{\circ} \mathrm{C}$, measuring temperature coefficient of resistivity $\alpha=0.0024 \mathrm{~K}^{-1}$ and sheet resistance $R_{o, s}=0.83 \Omega \cdot \square^{-1}$ for the $200 \mathrm{~nm}$ Pt sputtered layer. The sensor resistors are placed in a Wheatstone bridge, applying a voltage $V_{s r}$ of $0.1 \mathrm{~V}$, where a differential output voltage $\Delta V$ is obtained, which is nearly linearly proportional to the resistance change of both sensor resistors.

\subsection{Experimental and Model Results}

Figure 4.11a shows the measured resistance change for water flow rates from $0 \mu \mathrm{l} \cdot \mathrm{min}^{-1}$ up to $2 \mu \mathrm{l} \cdot \mathrm{min}^{-1}$. The average change in temperature of the heater, obtained from the numerical models, is plotted for comparison. The average temperature 
measured by the sensor structures is for a small part determined by the contact leads, because the heater and sensor resistors are not measured using four-point contacts. Figure $4.11 \mathrm{~b}$ shows the simultaneously measured differential voltage output of the Wheatstone bridge compared to numerical results. As expected, the heater tempera-

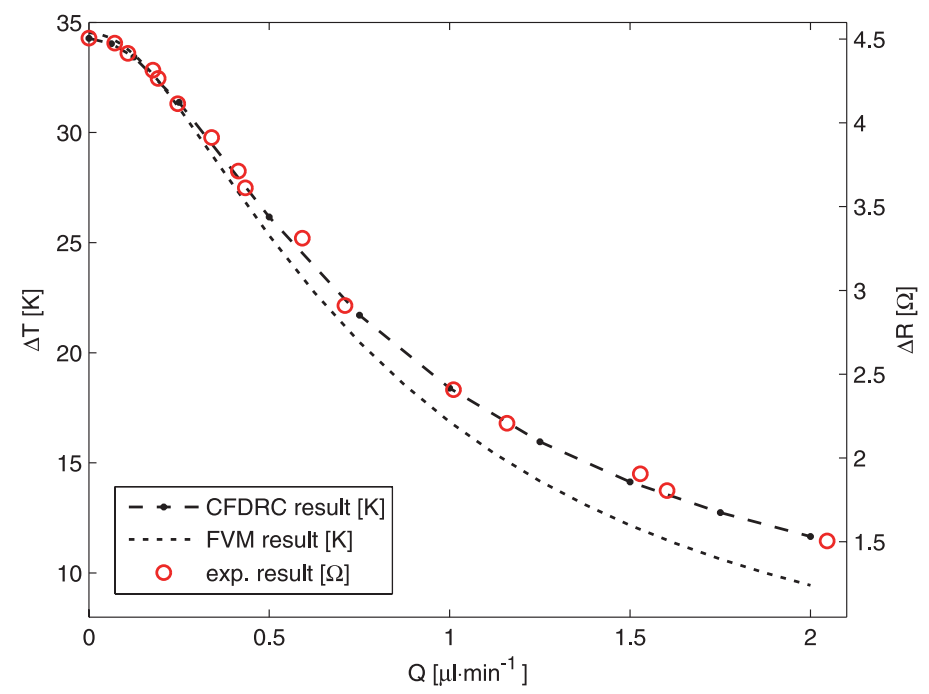

a)

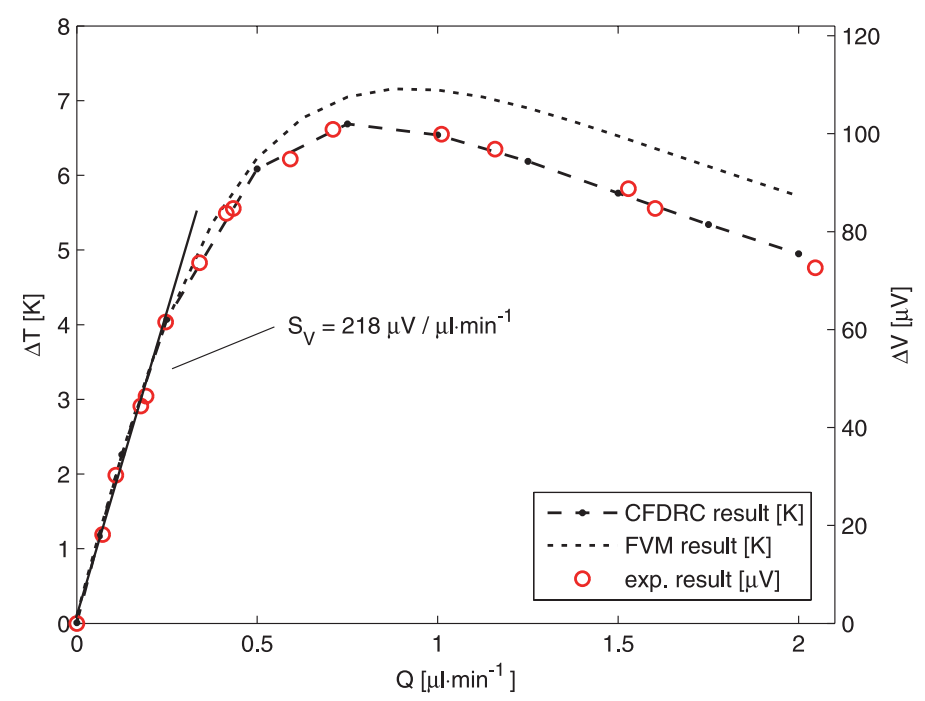

b)

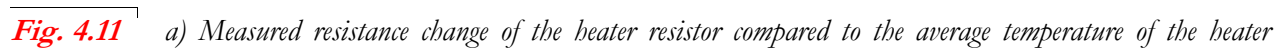
obtained from the numerical models and b) measured voltage output of the Wheatstone bridge compared to the average differential temperature between both sensor resistors obtained from the numerical models. 
ture is nearly constant at small flow rates with a linear sensor response $\Delta V$ having a flow sensitivity of $S_{V}=218 \mu \mathrm{V} / \mu \mathrm{l} \cdot \mathrm{min}^{-1}$. The sensor output remains linear up to flow rates in the order of $300 \mathrm{nl} \cdot \mathrm{min}^{-1}$.

The experimental and numerical results are in close agreement. However, $R_{0}$ in the numerical models is calculated from sheet-resistance and mask-layout data, while the actual $R_{0}$ of the heater and sensor resistors is somewhat different and is slightly influenced by temperature. Therefore, the experimental results were scaled to fit the numerical data. Obtained values of $\mathrm{R}_{0}$ after scaling are close to expected values from mask-layout and sheet-resistance data as well as four-point resistance measurement of the heater resistor between bondpads. The experimental data fits accurately to CFDRC results and differs only at higher flow rates for the FVM model.

Figure 4.12 shows additional measurements for water flow below $300 \mathrm{nl} \cdot \mathrm{min}^{-1}$. A fluid flow restriction was used in the measurement setup for flow rates below $100 \mathrm{nl} \cdot \mathrm{min}^{-1}$, allowing to measure at higher pressures, with higher calibration precision in determining the flow rate. Changes to the fluidic connection tubes introduced a systematic error in flow rate accuracy giving a different sensor sensitivity $S_{V}=203 \mu \mathrm{V} / \mu \mathrm{l} \cdot \mathrm{min}^{-1}$ compared to measurement results in Fig. 4.11 . The flow rate became unstable for pressures approaching zero, which was found to be the limiting factor in measuring liquid flow rates below $40 \mathrm{nl} \cdot \mathrm{min}^{-1}$, with the pressure driven flow setup used.

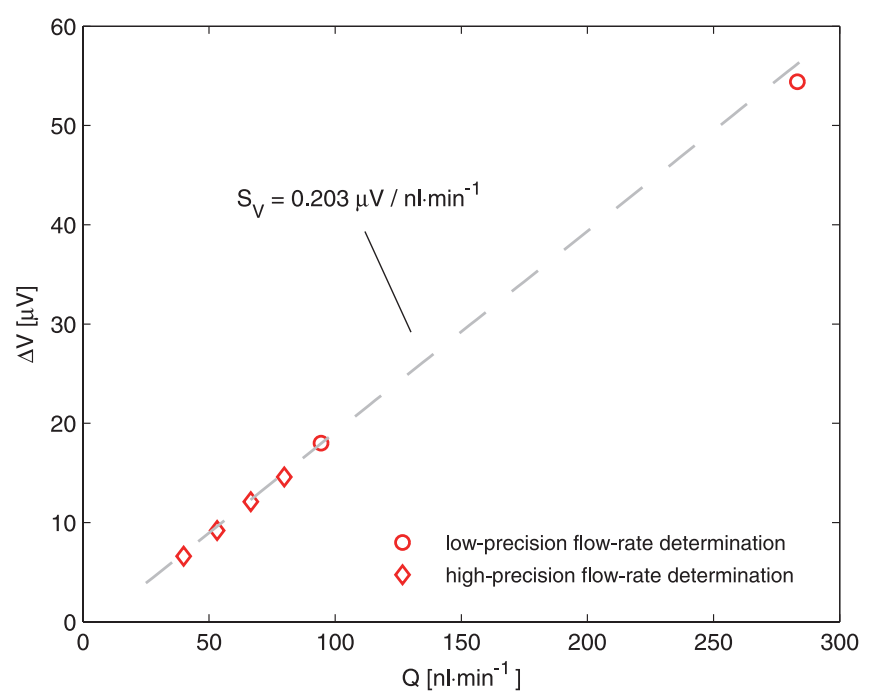

Fig. 4.12 Linear sensor output for water flow below $\mathrm{nl} \cdot \mathrm{min}^{-1}$, with $\tau_{\text {sample }}=1 \mathrm{~s}$. A flow restriction was used to determine the flow rate, with higher calibration precision below $100 \mathrm{nl} \cdot \mathrm{min}^{-1}$. 


\subsection{Conclusions}

A miniaturised calorimetric flow sensor was realised using a versatile surface channel concept, demonstrating the capability of the technology for use in thermal flow sensor applications, where the microchannel is thermally isolated from the substrate. The concept allows for the fabrication of microchannels with low hydraulic resistance and total fluid volume, directly below the substrate surface, while sensor structures are deposited on a planar substrate surface avoiding step-coverage problems. The surface microchannel technology can be used in the integration of other sensor materials, like materials with high temperature coefficient of resistivity $[4.3,4.4]$ or the integration of thermopiles.

A linear differential calorimetric sensor response was measured for water flow up to flow rates in the order of $300 \mathrm{nl} \cdot \mathrm{min}^{-1}$. The measured sensor output is in close agreement with results obtained from the CFDRC model and the approximate FVM model of the sensor, with the FVM model giving direct accurate results in the linear regime of the calorimetric flow sensor. The highly-accurate CFDRC model shows that the conduction through the surrounding air is significant for the calculation of the temperature profiles along the microchannel. The output of the sensor and the heater temperature give the typical response of a calorimetric flow sensor, however the CFDRC model shows that this is not due to a thermal boundary layer in the fluid. It is through the asymmetric temperature field in the surrounding air by advection of heat in the microchannel, partially measured by the contact leads, that the sensor obtains a King's law behaviour.

The FVM model is sufficiently accurate to be used with other sensor designs. The Pt contact leads resulted in a radial heat loss, which is significant close to the microchannels and can be avoided by running the leads over the microchannel.

\section{References}

[4.1] M. Dijkstra, M.J. de Boer, J.W. Berenschot, T.S.J. Lammerink, R.J. Wiegerink, M. Elwenspoek, "Miniaturized thermal flow sensor with planar integrated sensor structures on semicircular surface channels", Sensor. Actuat. A-Phys., 143 (2008) 1-6.

[4.2] S. Wu, Q. Lin, Y. Yuen, Y-C. Tai, "MEMS flow sensors for nano-fluidic applications", Sensor. Actuat. A-Phys., 89 (2001) 152-158.

[4.3] Y. Mizuno, M. Liger, Y-C. Tai, "Nanofluidic flowmeter using carbon sensing element", Proc. IEEE MEMS, (2004) 322-325.

[4.4] M. Dijkstra, T.S.J. Lammerink, R.J. Wiegerink, M. Elwenspoek, "Nano-flow thermal sensors applying dynamic $\omega-2 \omega$ sensing method", Proc. MME, (2006) 29-32.

[4.5] N.R. Tas, T.S.J. Lammerink, P.J. Leussink, J.W. Berenschot, H-E. de Bree, M. Elwenspoek, "Towards thermal flowsensing with $\mathrm{pl} / \mathrm{s}$ resolution", Proc. SPIE, 4176 (2000) 106-121. 
[4.6] M. Dijkstra, M.J. de Boer, J.W. Berenschot, T.S.J. Lammerink, R.J. Wiegerink, M. Elwenspoek, "A versatile surface channel concept for microfluidic applications", J. Micromech. Microeng., 17 (2007) 1971-1977.

[4.7] M.J. Kim, L.A. Gruenke, R.J. Saia, S.S. Cohen, "Inhibition of acid etching of Pt by pre-exposure to oxygen plasma”, Appl. Phys. Lett., 44 (1984) 462-464.

[4.8] CFD Research Corp. - bttp://www.cfdrc.com.

[4.9] The MathWorks, Inc. - bttp:// www.matworks.com.

[4.10] H.K. Versteeg, W. Malalasekra, "An introduction to computational fluid dynamics, The finite volume method", Prentice Hall, Harlow, $1^{\text {st }}$ edn. (1995). 


\section{Low-Drift Flow Sensor with Thermopile-Based Power Feedback}

A thermal flow sensor has been realised consisting of freely suspended silicon-rich silicon-nitride microchannels with an integrated Al/poly-Si $i^{++}$thermopile in combination with up- and downstream Al heater resistors. The zero offset of a thermopile at uniform temperature is exploited in a feedback loop controlling the dissipated powers in the heater resistors, minimising inevitable influences of resistance drift, mismatch of thin-film metal resistors and thermopile material drift. The control system cancels the flow-induced temperature difference across the thermopile by controlling a power difference between both heater resistors, thereby giving a measure of the flow rate. The flow sensor was characterised for power difference versus water flow rates up to $1.5 \mu \mathrm{l} \cdot \mathrm{min}^{-1}$. Results are in good agreement with a thermal model of the sensor, and the low-drift operation of the temperature-balancing control system was verified. ${ }^{5.1}$.

\subsection{Introduction}

The miniaturisation of microfluidic components in chemical analysis, synthesis, biotech and nanotechnology asks for the accurate and reliable measurement of tiny fluid flow rates. Current micromechanical thermal flow sensors are capable of measuring liquid flows down to a few $\mathrm{nl} \cdot \mathrm{min}^{-1}[5.1,5.2]$. However, the long-term measurement accuracy of these sensors is ultimately limited by drift in the properties of temperature sensing and heating elements, for which thin-film layers are commonly used $[5.3,5.4]$. Alternatively, thermopiles can be used as differential temperature sensors [5.5-5.8].

This chapter discusses a thermal flow sensor and control system that exploits the low offset-drift differential-temperature dependent output $V_{T C}$ of a thermopile [5.9] in order to obtain a flow sensor that is independent of resistance drift and mismatch of thin-film metal resistors used (Fig. 5.1). The dissipated powers $P_{1}, P_{2}$ in the heater resistors up- and downstream from the thermopile are determined and controlled independently of resistance values by measuring both the current through and the voltage across the resistors, thereby eliminating influences of resistance drift. Additionally, the control-system feedback loop minimises the dependency on non-

\footnotetext{
5.1 This chapter is based on an article accepted for publication in J. Microelectromech. S.
} 
linear thermopile characteristics $m_{T C}\left(\Delta T_{T C}\right)$ and drift in thermocouple material properties by cancelling the generated output voltage $V_{T C}$.

The output voltage $\mathrm{V}_{T C}$ and temperature difference across the thermopile is cancelled by controlling a power difference $\Delta P$ between the two heater resistors, with a fixed amount of total power $P_{T}$ being dissipated, according to the temperaturebalancing anemometry principle [5.10,5.11]. Applying a fluid flow changes the temperature distribution around the heaters by forced convection. This results in the relation $\Delta P / P_{T}$ being dependent on the flow rate $Q$. A measure of the flow rate $m_{T B}\left(\Delta P / P_{T}\right)$ can be obtained, with $\Delta P / P_{T}$ being linear for small flow rates.

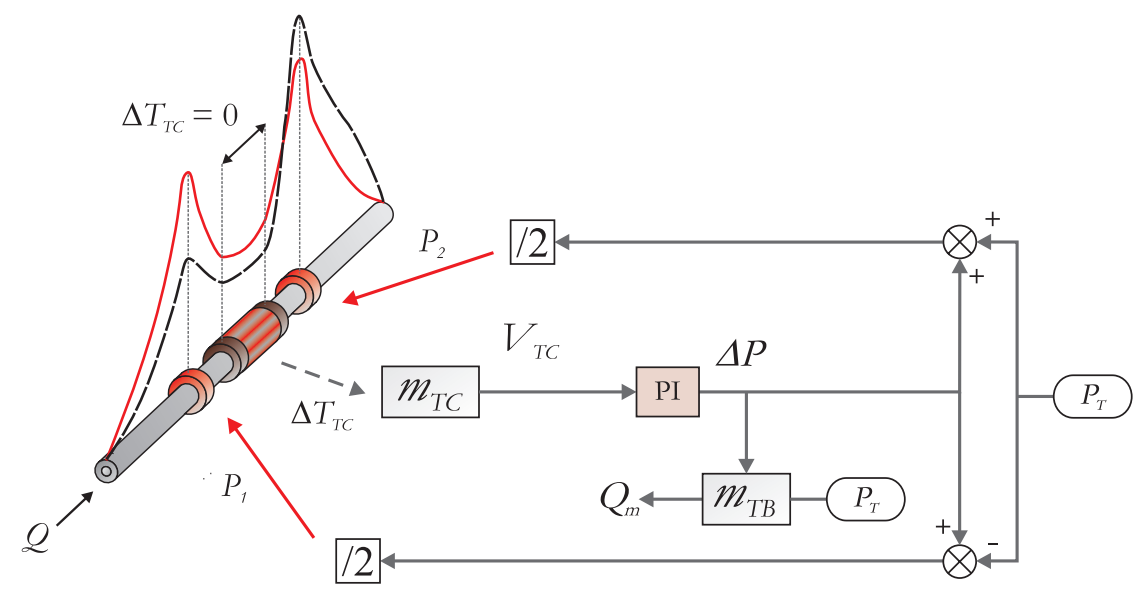

Fig. 5.1 Thermopile-based temperature-balancing flow sensor.

Ultimately, the proposed measurement concept can be realised using standard electronics, $\mathrm{AD} / \mathrm{DA}$-converters and a microcontroller. This can lead to fully integrated flow sensors with improved accuracy for flow rates in the nl. $\mathrm{min}^{-1}$ range. In this chapter results of liquid flow measurements on a fabricated thermopile flow sensor using a temperature-balancing control system are discussed and compared with thermal model calculations. Long-term drift performance is analysed, where the effect of long-term drift in material properties is observed to be cancelled in the sensor output signal. Other influences on flow sensor output drift are also discussed.

\subsection{Sensor Fabrication}

The fabricated thermopile flow sensor incorporates mechanically-strong freely suspended surface microchannels for on-chip transport of tiny amounts of fluid flow. Surface microchannels are fabricated without the requirement for a sacrificial layer 
and allow for the integration of sensor elements in close proximity to the fluid [5.12] as explained in Chap. 3.

Figure 5.2 gives a schematic overview of the process scheme for the fabrication of the low-drift thermopile flow sensor. Surface microchannels are created by isotropic dry etching, using high-density $\mathrm{SF}_{6}$ plasma with zero self-bias. The microchannels are etched through etch holes $2 \mu \mathrm{m}$ in width, in a low-stress $500 \mathrm{~nm}$ silicon-rich siliconnitride (SiRN) layer. The etch holes and inner surfaces of the microchannels are conformally coated by a second low-stress LPCVD deposited $1.3 \mu \mathrm{m}$ SiRN layer, resulting in completely sealed microchannels, while leaving a planar substrate surface for the integration of an $\mathrm{Al} /$ poly-Si ${ }^{++}$thermopile and $\mathrm{Al}$ heater resistors. The thermopile is created by LPCVD deposition and boron doping by solid source diffusion of a $200 \mathrm{~nm}$ poly-Si layer and sputtering of a $200 \mathrm{~nm}$ Al layer (Fig. 5.2a).
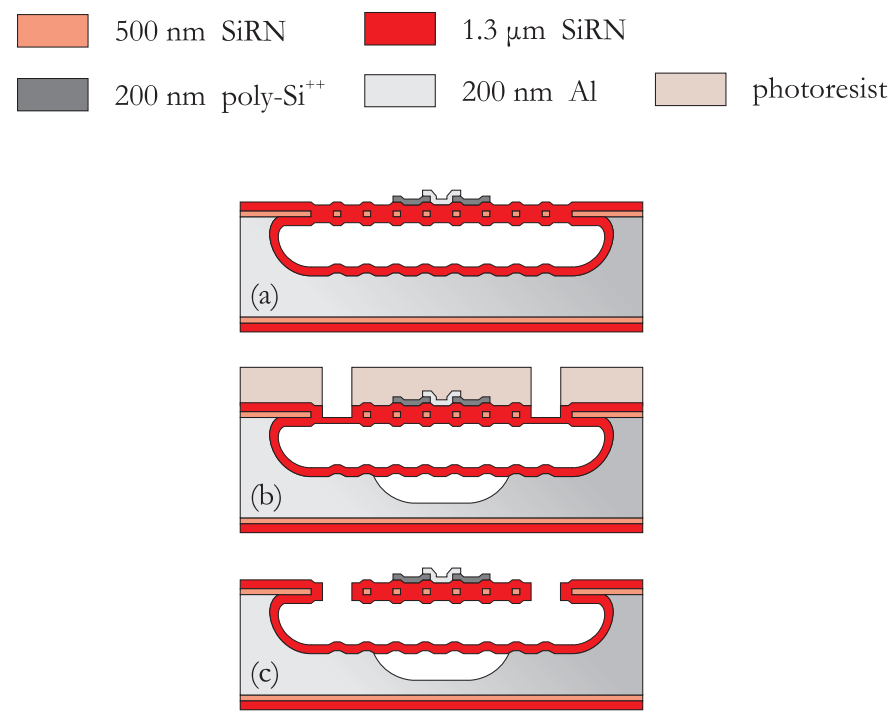

Fig. 5.2 Process scheme for the fabrication of the microchannel thermopile flow sensor.

The surface microchannels are released by $\mathrm{SF}_{6}$ plasma etching, for thermal isolation from the heat-conducting substrate, with the photoresist mask protecting the sensor elements during the release (Fig. 5.2b). The photoresist is removed after fluidic entrance holes are etched through the SiRN layer, allowing for direct interfacing to the microchannels on the substrate surface.

Figure 5.3 shows SEM micrographs of the fabricated thermopile flow sensor. The sensor contains five parallel $20 \mu \mathrm{m}$ diameter microchannels, spanning a $1.6 \mathrm{~mm}$ long thermal isolation cavity. The parallel microchannels have $200 \mu \mathrm{m}$ total width, which allows for the integration of an $\mathrm{Al} /$ poly-Si $^{++}$thermopile (Fig. 5.4) with 26 junctions 


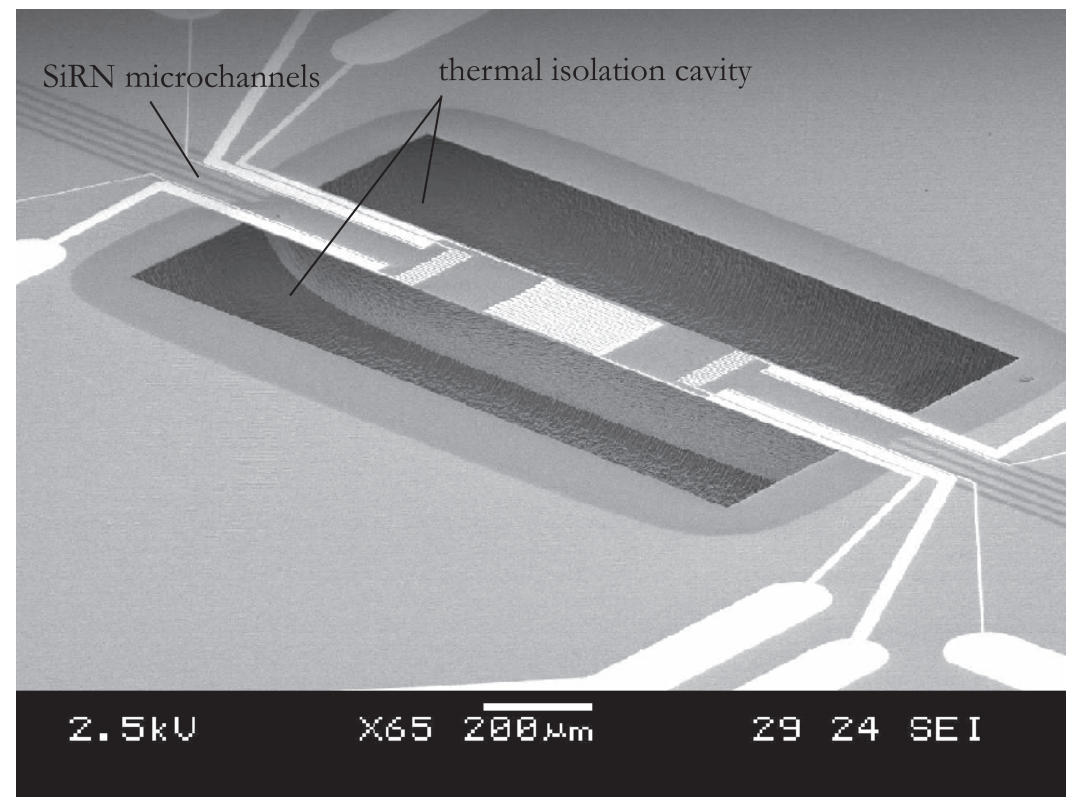

a)

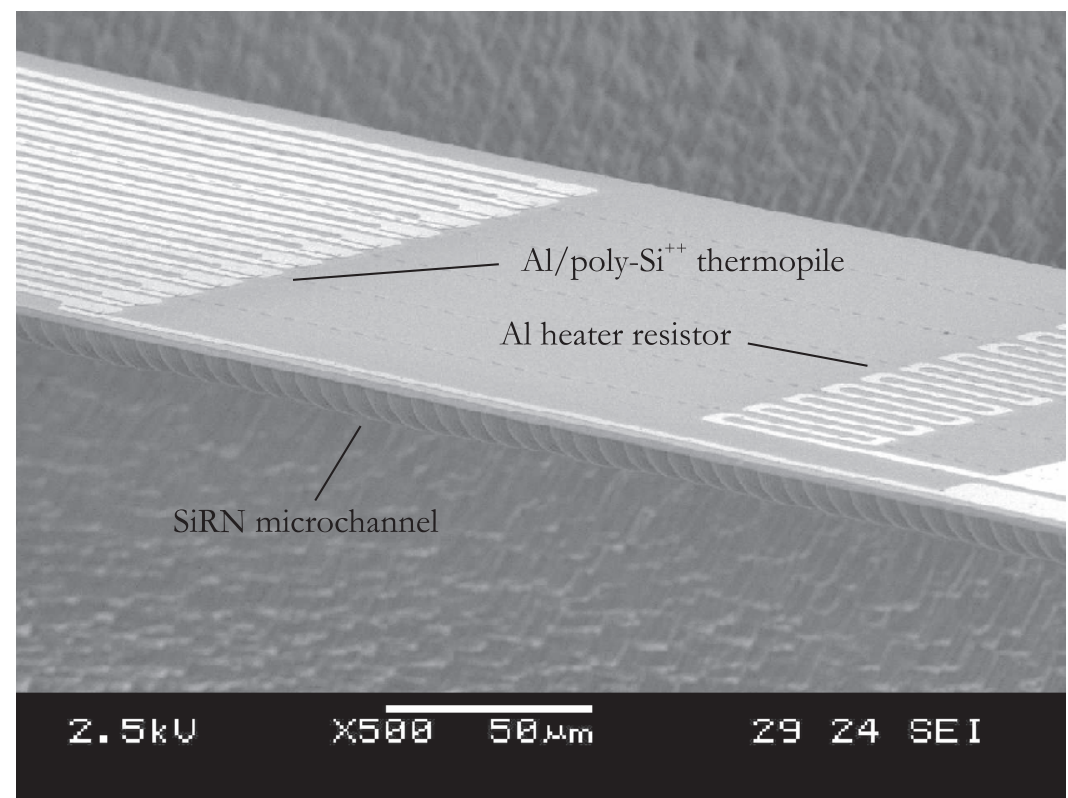

b)

Fig. 5.3 Microchannel flow sensor with integrated $A l$ heater resistors and $A l / p o l y-S i^{++}$thermopile. 


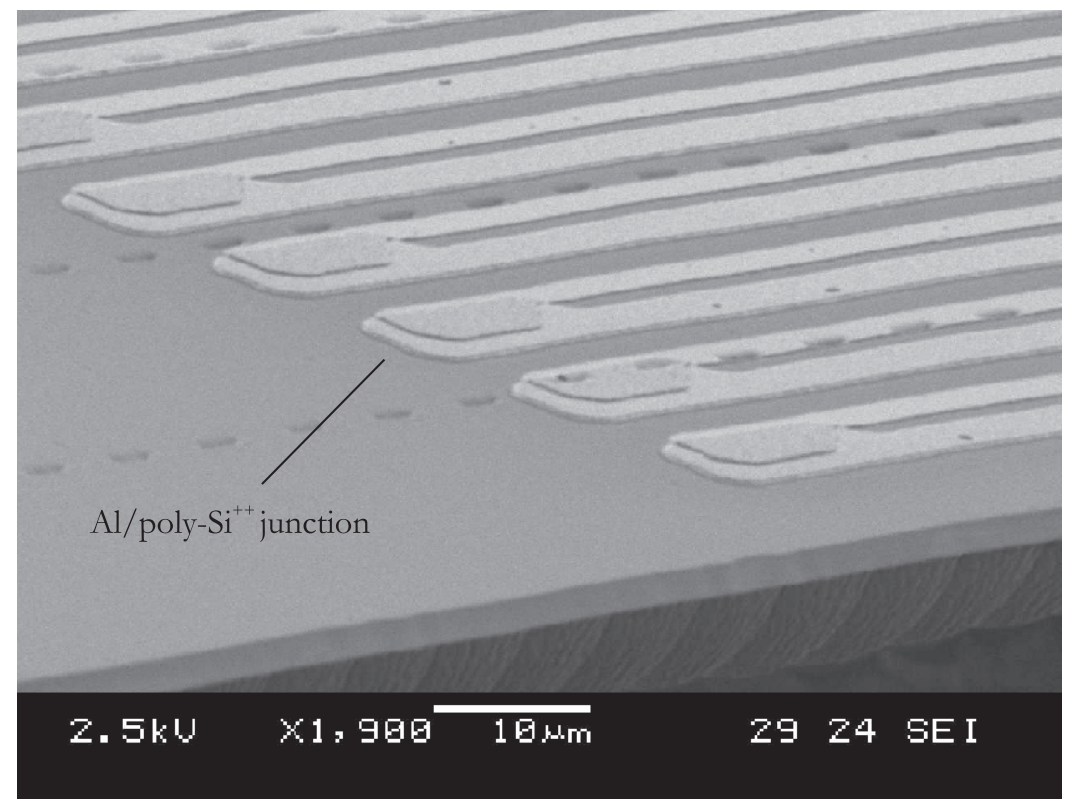

Fig. 5.4 Integration of Al/poly-Si++ thermopiles on microchannel membrane.

and up- and downstream $\mathrm{Al}$ heater resistors. The $\mathrm{Al}$ heater resistors have four-point measurement contacts for accurate resistance measurements.

\subsection{Sensor Modelling}

A two-dimensional cylindrical finite volume method (FVM) thermal model [5.13] of the flow sensor was constructed in MATLAB. The model gives approximate solutions to the heat advection-diffusion equation (5.1) solving the temperature field $T$ surrounding the freely-suspended microchannel, taking into account the thermal conductivities $\kappa$ of the freely-suspended microchannel containing fluid, the sensor elements, electrical connection leads and surrounding air. Ambient temperature is imposed as a boundary condition at a radius of $450 \mu \mathrm{m}$ from the microchannel, modelling the under-etched thermal isolation cavity. A fully-developed fluid flow profile $v_{x}$ is modelled inside the microchannel along the $x$-direction, with fluid density $\rho$ and fluid specific heat capacity $c$. The heater elements are modelled by a heat-flow source term per unit volume $P^{\prime \prime \prime}$.

$$
\left(c \rho v_{x} \frac{\partial}{\partial x}-\nabla(\kappa \nabla)\right) T=P^{\prime \prime \prime}
$$


Equation (5.1) can be solved on a cylindrical mesh of the flow sensor applying Gauss theorem. This results in a sparse-matrix equation (5.2), with convection matrix GQ, volume flow rate $\mathcal{Q}$ and conduction matrix $\mathbf{G}$, where $\vec{T}_{e}$ and $\vec{P}_{e}$ are column vectors of respectively the temperature and heat-flow source term of each element in

$$
\begin{array}{r}
(\mathcal{Q} \cdot \boldsymbol{G}-\boldsymbol{G} \boldsymbol{\kappa}) \vec{T}_{e}=\vec{P}_{e} \\
\boldsymbol{G} \vec{T}_{e}=\vec{P}_{e}
\end{array}
$$

the cylindrical mesh. Equation (5.2) can directly be solved in MATLAB, using the Gauss-Seidel method.

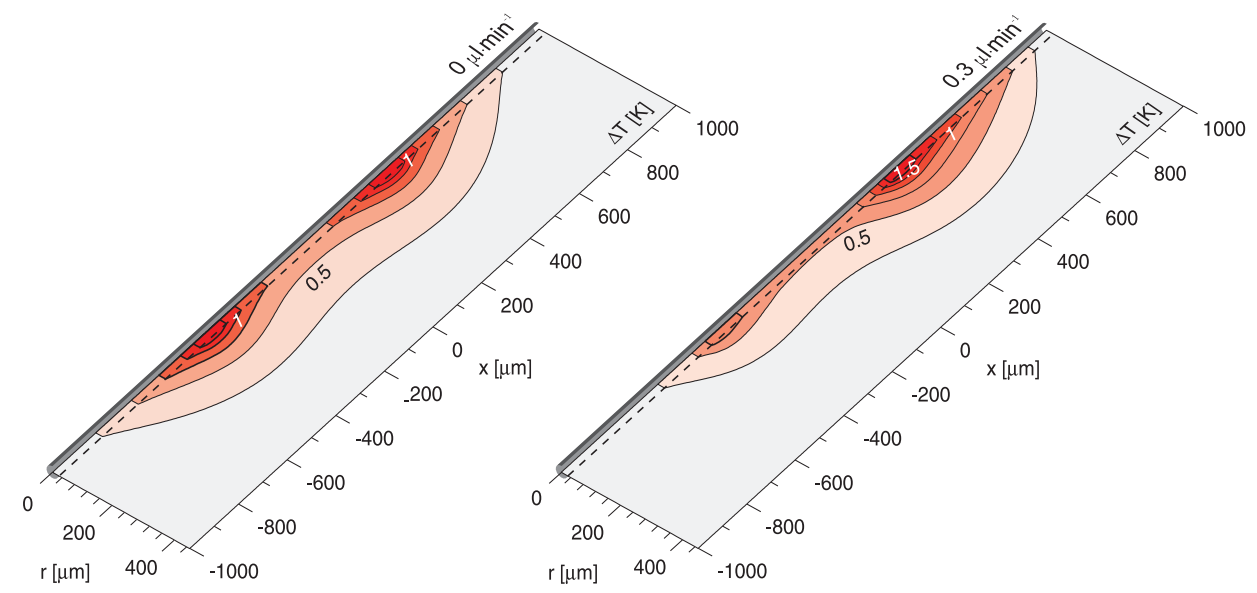

a)

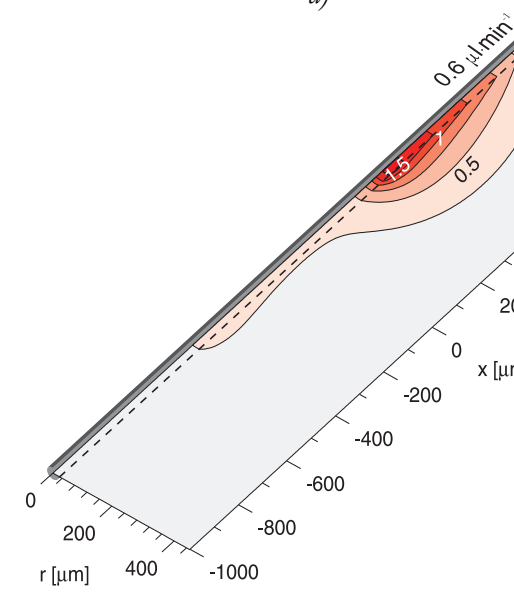

c) b)
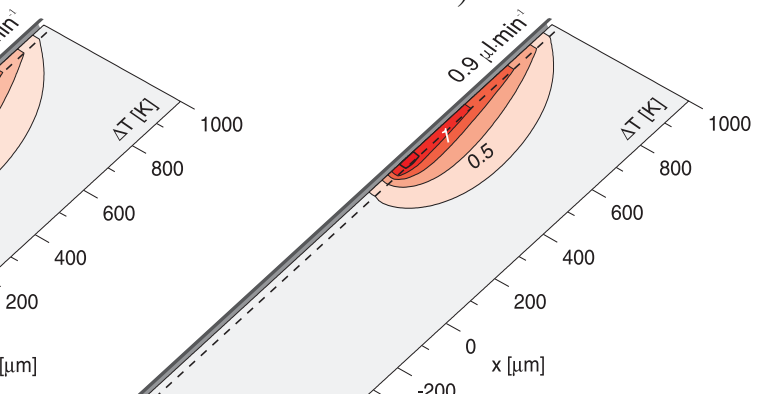

Fig. 5.5 FVM thermal model result, showing the temperature field at various water flow rates in positive $x$-direction, with $0.1 \mathrm{~mW}$ total power $P_{T}$. 
The model allows for fast successive iteration at a given flow rate in order to find the power difference $\Delta P$ required for both sides of the thermopile to be at the same temperature, in accordance with the temperature-balancing measurement concept [5.10].

Figure 5.5 shows the temperature field at $0.3 \mu \mathrm{l} \cdot \mathrm{min}^{-1}$ water flow, with $0.1 \mathrm{~mW}$ total power dissipation. Several temperature profiles, along the freely-suspended microchannel, were obtained from model calculations at different flow rates (Fig. 5.6).

The temperature profile at $0 \mu \mathrm{l} \cdot \mathrm{min}^{-1}$ is symmetric, with both heaters dissipating equal amount of power. The temperature profile around the heaters is disturbed by advection of heat on applying water flow through the microchannel. Maintaining zero temperature difference between both sides of the thermopile $\Delta T_{T C}$ requires the upstream heater to dissipate less power than the downstream heater.

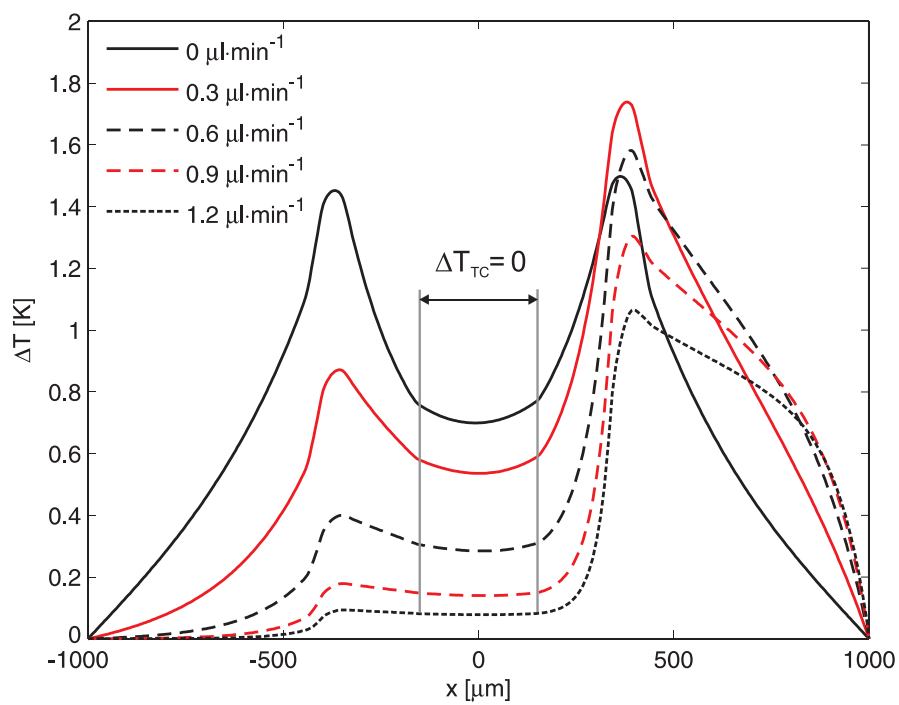

Fig. 5.6 FVM model calculation results giving temperature profiles along the freely-suspended microchannel, with water flow in the positive $x$-direction.

The upstream heater temperature decreases with increasing flow rate and decreasing heating power. However, the downstream heater does not increase in temperature for water flow rates higher than $0.6 \mu l \cdot \mathrm{min}^{-1}$, due to the total power $P_{T}$ being limited, while a large amount of heat is advected from the heater, limiting the sensitivity of the flow sensor for flow rates higher than $0.6 \mu \mathrm{l} \cdot \mathrm{min}^{-1}$. 


\subsection{Experimental Results}

Thermopile flow sensor chips were fabricated $12.5 \times 12.5 \mathrm{~mm}$ in size, with fixed bondpad and microchannel entrance hole positions for self alignment with a chip holder, containing O-rings for fluidic interfacing and twelve pogo-pins for electrical connections to the sensor chip (Fig. 5.7).

An elevation head up to a few meters $\Delta h$ was used to force water through the thermopile flow sensor (Fig. 5.8). The flow is restricted to a few $\mu l \cdot \mathrm{min}^{-1}$ by the hydraulic resistance of the surface microchannels. The elevation head gives a stable flow rate, which is determined by weighing. The volume flow rate is calculated by taking the time-derivative of the amount of liquid flowing onto a scale, divided by the density of water.

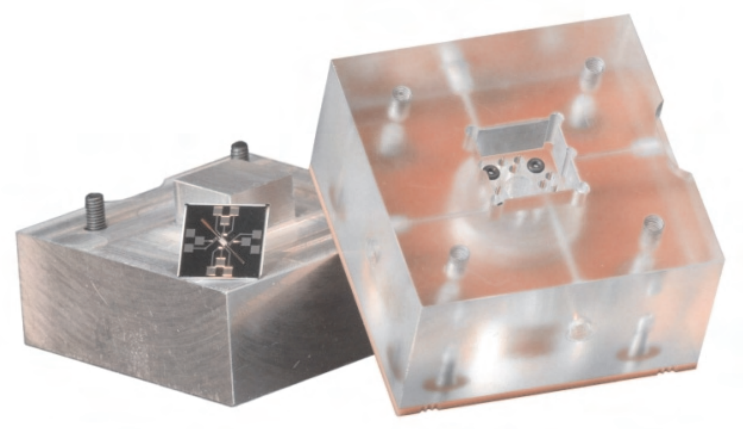

Fig. 5.7 Chip holder for fluidic and electrical interfacing with the sensor chip. The sensor chip measures $12.5 \times$ $12.5 \mathrm{~mm}$.

The power feedback loop and temperature-balancing controller were implemented in Matlab. A Hewlett Packard 34401A multimeter was used to measure the thermopile output voltage $V_{T C}$. The heating powers $P_{1}, P_{2}$ dissipated in the resistors are controlled by a Keithley 2602 two-channel source measurement unit (SMU). The SMUs measure the voltage across the heater resistors and the dissipated power is controlled by adjusting the current through the resistors. In this way the exact resistance value is not important and may drift.

The total heating power $P_{T}$ was kept constant at $0.1 \mathrm{~mW}$ during these experiments, resulting in a temperature rise of a few Kelvin of the heater resistors with respect to the substrate. The difference in heating power $\Delta P$ needed to cancel the thermopile output voltage $V_{T C}$ was measured for applied water volume flow rates $Q$ up to $1.5 \mu \mathrm{l} \cdot \mathrm{min}^{-1}$. Figure 5.9 shows the measured $\Delta P / P_{T}$ ratio, together with results obtained from FVM model calculations. FVM model and measurement results show 


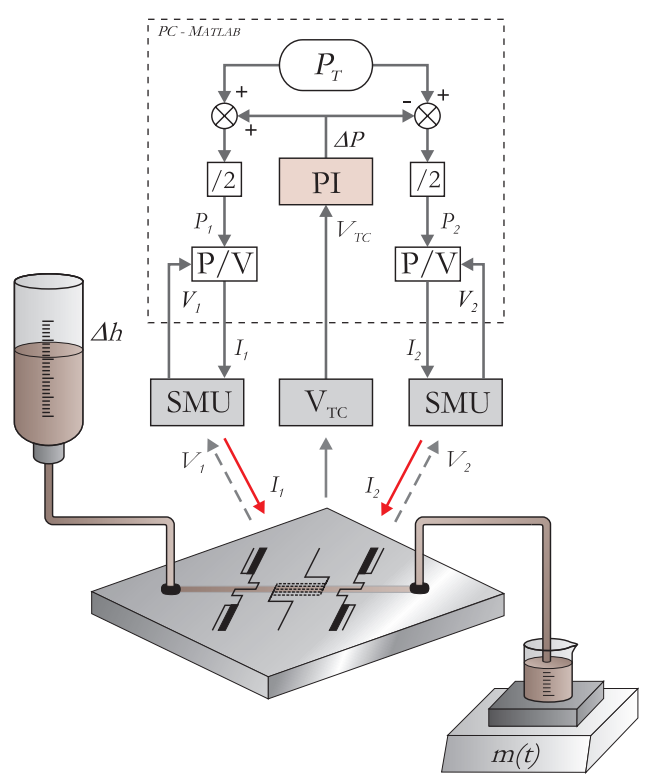

Fig. 5.8 Schematic overview of experimental setup, applying temperature-balancing power feedback control with $\Delta P / P_{T}$ being the flow dependent sensor output.

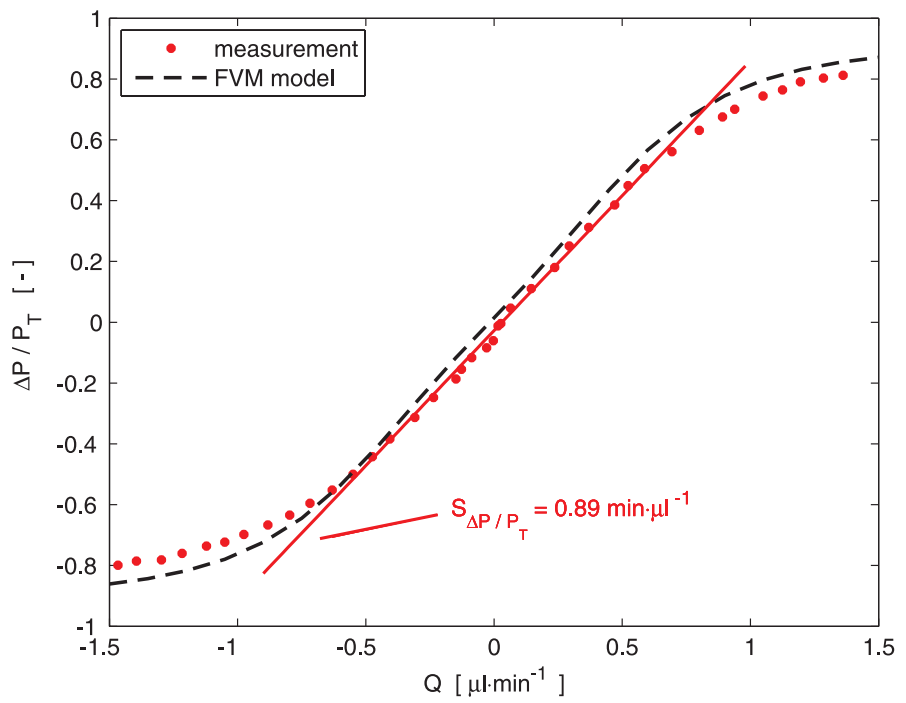

Fig. 5.9 Measured sensor output $\Delta P / P_{T}$ as a function of water volume flow rate $Q$. The total heating power $P_{T}$ is kept constant at $0.1 \mathrm{~mW}$. 
the sensor having a linear response, with $0.89 \mu \mathrm{l} \cdot \mathrm{min}^{-1}$ sensitivity $S_{\Delta P / P_{T}}$, up to about $0.5 \mu \mathrm{l} \cdot \mathrm{min}^{-1}$.

Figure 5.10 shows the control system response on applying a stepwise changing volume flow rate. It shows the measured heating powers $P_{1}, P_{2}$ being adjusted in response to the flow. Correct operation of controlling the dissipated powers in the heater resistors is demonstrated by the fact that a change in the resistance values $R_{1}$ and $R_{2}$, mostly due to a change in $\Delta P$, is not visible in the output signal. The output signal $\Delta P / P_{T}$ is a measure of the flow rate, with the thermopile output voltage $V_{T C}$ being controlled back to zero after each step in the flow rate. The discrete-sampling time of the controller is mostly determined by the integration time required to sample the thermopile voltage down to the required precision. The settling time of the PI controller required to adjust to a new flow rate is about $30 \mathrm{~s}$, which is too slow for thermal flow sensor applications, although PI controller settings were not fully optimised. A state-free controller can be used, based on a linear least square method,
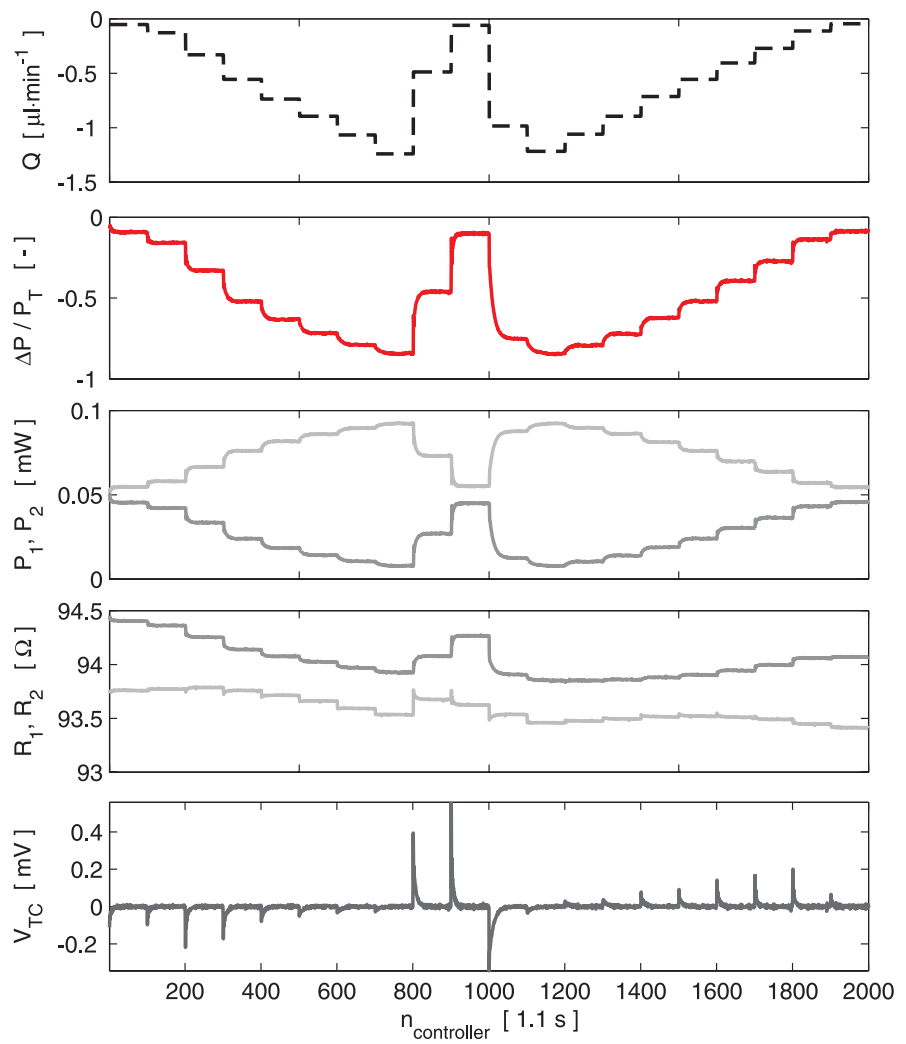

Fig. 5.10 Measured sensor control system response with changing flow rate. The total heating power $P_{T}$ is kept constant at $0.1 \mathrm{~mW}$. 
which would require at least two thermopile voltages $V_{T C}$ to be measured at different power differences $\Delta P$.

The low-drift operation of the temperature-balancing control system was analysed in an ambient surrounding by controlling $\Delta P$ for $13 \mathrm{hrs}$ with no flow applied, while dissipating $1 \mathrm{~mW}$ total power $P_{T}$. A Hewlett Packard 34420A nanovolt meter was used to measure the thermopile output voltage $V_{T C}$. Figure 5.11 shows obtained normalised power spectral densities. The power spectral densities of the average of both heater resistors $R_{\mu}$ and the difference between both heater resistors $R_{\Delta}$ show a $1 / f^{\alpha}$ slope. The drift in $R_{\mu}$ can mostly be attributed to fluctuations in ambient temperature, while drift in $R_{\Delta}$ is caused by degradation (Sec. 2.2.1). The integral action of the PI controller cancels low frequency components in the thermopile output voltage $V_{T C}$, resulting in a smaller power spectral density at lower frequencies. The power spectral density for $\Delta P$ is nearly flat, where the power control on the heater resistors is most effective in reducing output drift of the flow sensor.

The power difference $\Delta P$ shows an offset, which can be explained by heatconduction asymmetries in the sensor, internal offsets in the measurement equipment and thermocouple offset voltages on contact lead and bondwire leads required for electrical interfacing to the sensor chip and due to thermopile material irregularities. As well as a non-uniform Seebeck profile along the leads of the thermopile. The thermocouple voltages on the heater resistors are compensated by the control system, by averaging the voltage measured across the resistor, while alternating the direction

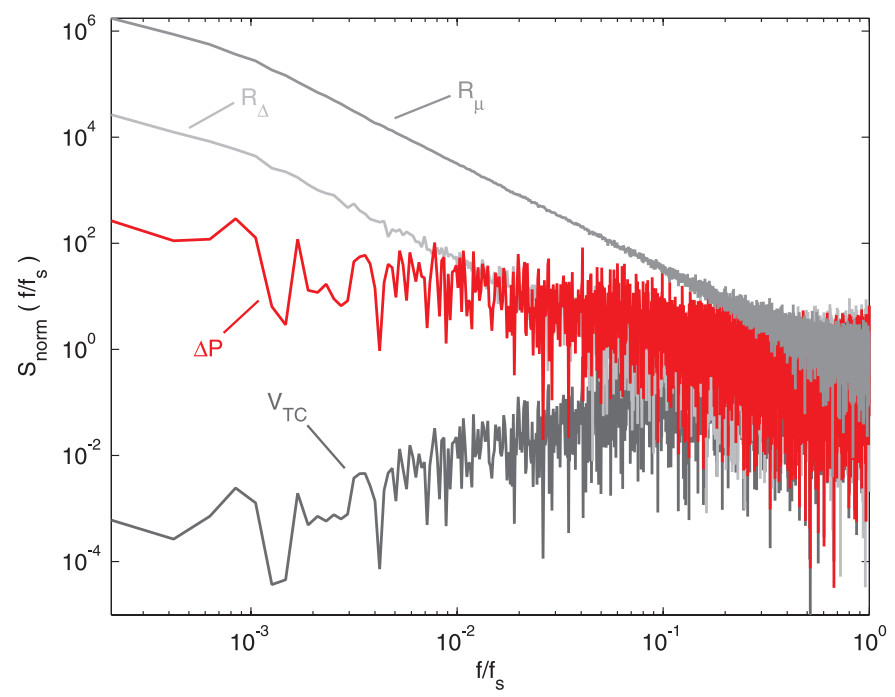

Fig. 5.11 Drift measurement for 13 hrs with $0.24 \mathrm{~Hz}$ sampling frequency $f_{\text {s, }}$ showing normalised power spectral density $S_{\text {norm }}$ at $1 \mathrm{~mW}$ total power $P_{T}$. 
of the current. The Hewlett Packard 34420A nanovolt meter has low offset drift, but alternatively a chopper opamp or zero-drift opamp (AD8628) can be integrated close to the sensor chip minimising amplifier offset drift. Influence of offset voltages by thermocouple interface leads to the sensor chip can be reduced by increasing the amount of junctions in the thermopile.

The offset in power difference $\Delta P$ was analysed further by repeating the long term drift experiment in an oven at $90{ }^{\circ} \mathrm{C}$ average temperature for $147 \mathrm{hrs}$. Also the drift in the thermopile sensitivity was measured independently from the feedback loop. Figure 5.12 shows measurement results, where the average heater resistance value $\Delta R \mu$ is slowly changing by a $2{ }^{\circ} \mathrm{C}$ decrease in oven temperature. The difference between the resistance values $\Delta R_{\Delta}$ shows the influence of induced degradation at an elevated temperature. The power difference $\Delta P$ for low frequencies does not correlate to changes in heater resistance values as well as drift in thermopile sensitivity, demonstrating the correct low-drift operation of the temperature-balancing control system. The power difference $\Delta P$ for relatively high frequencies shows to have high correla-
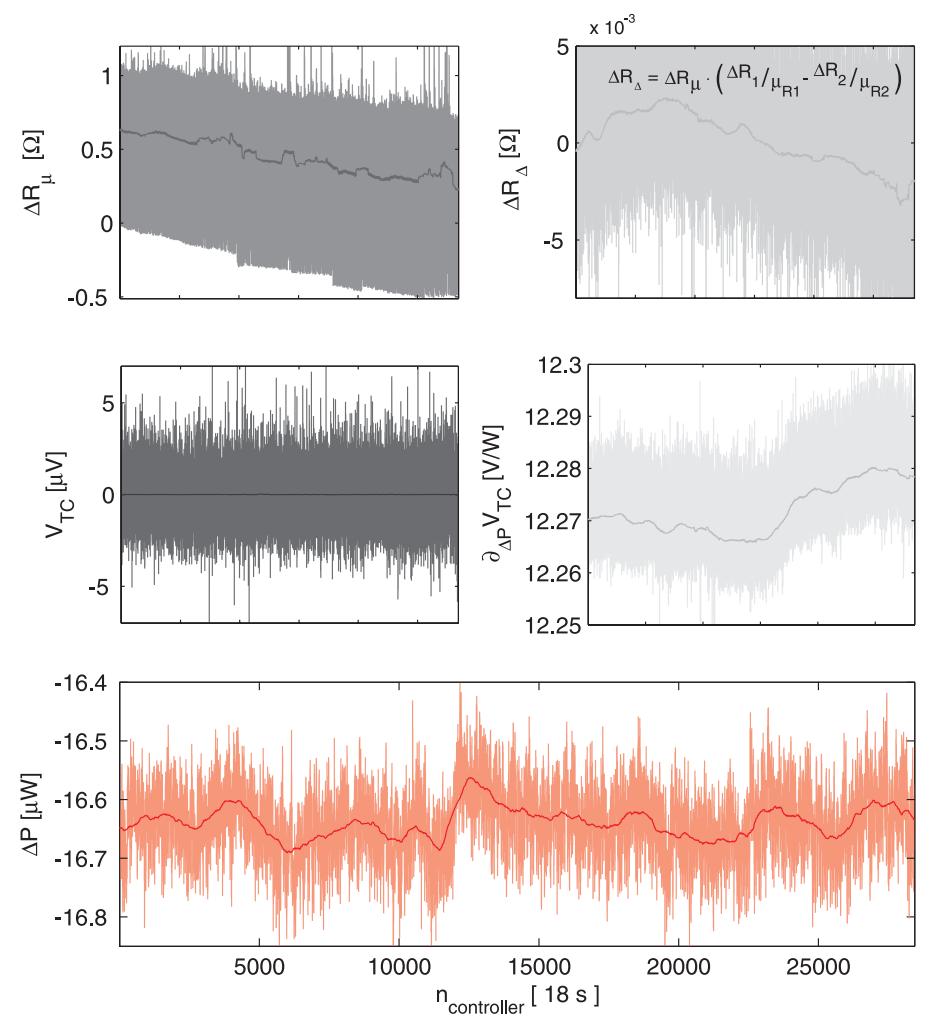

Fig. 5.12 Drift measurement for 147 hrs at $90{ }^{\circ} \mathrm{C}$ average oven temperature, while dissipating $0.1 \mathrm{~mW}$ total power $P_{T}$ with 1000 pnt. moving average. 
tion to the difference between the resistance values $\Delta R \mu$ and, in lesser extend, to the drift in thermopile sensitivity. Indicating that, at $90{ }^{\circ} \mathrm{C}$ oven temperature, residual sensor output fluctuations are mainly caused by asymmetric temperature profile fluctuations on heater resistors and contact leads on the freely-suspended microchannel.

Measurements show that the flow sensor remains sensitive to temperature gradients across the sensor chip. This temperature gradient is modulated by oven temperature, due to spatial differences in heat conduction to ambient, caused by the connection leads going outside the oven. Temperature cycles in oven temperature therefore influence the power difference $\Delta P$. The fluctuations in power difference $\Delta P$ in Fig. 5.12 are around $0.3 \%$ of the total power $P_{T}$ dissipated and are mostly caused by the modulation of temperature gradients across the chip.

Measurements with increased total power $P_{T}$, inducing electromigration, show stable operation of the temperature-balancing control system. A change in power difference $\Delta P$ does however occur in the extreme case that voids are formed by electromigration. This can be explained by a change in the temperature profile on the heater resistor, due to a local increase in current density.

\subsection{Conclusions}

A thermally-isolated microchannel flow sensor has been fabricated, with integrated $\mathrm{Al} /$ poly-Si $^{++}$thermopile and up- and downstream $\mathrm{Al}$ heater resistors. Flow measurements and FVM model calculations have shown that a temperature-balancing control system, which cancels the flow-induced temperature difference across the thermopile by controlling a power difference between both heater resistors, can give a linear measure of water flow rates up to about $0.5 \mu \mathrm{l} \cdot \mathrm{min}^{-1}$. The temperature-balancing control system requires about $30 \mathrm{~s}$ settling time to adjust to a change in flow rate. The settling time can be avoided using a state-free controller. The discrete-sampling time of the controller being $1.1 \mathrm{~s}$ is limited by the integration time required to obtain the thermocouple voltage with sufficient precision. The differential-temperature dependent output voltage generated by the thermopile and power control on heater resistors has been exploited to minimise influences of drift in material properties. The sensor output drift which remains, is caused by temperature gradients across the sensor chip and by measurement errors. Temperature gradients have to be compensated for by sensor design. Measurement errors can be caused by interface thermocouple voltages, thermopile material irregularities or measurement offsets. A chopper or zero-drift opamp and a large number of thermopile junctions can improve the reduction in offset drift of the thermopile voltage due to interface lead thermo- 
thermocouple voltages. An offset in power difference $\Delta P$ can still remain, due to thermopile material irregularities and flow-sensor heat-conduction asymmetries to the substrate.

\section{References}

[5.1] M. Dijkstra, M.J. de Boer, J.W. Berenschot, T.S.J. Lammerink, R.J. Wiegerink, M. Elwenspoek, "Miniaturized thermal flow sensor with planar integrated sensor structures on semicircular surface channels", Sensor. Actuat. A-Phys., 143 (2008) 1-6.

[5.2] S. Wu, Q. Lin, Y. Yuen, Y-C. Tai, "MEMS flow sensors for nano-fluidic applications", Sensor. Actuat. A-Phys., 89 (2001) 152-158.

[5.3] P. Bruschi, D. Navarrini, M. Piotto, "A flow sensor for liquids based on a single temperature sensor operated in pulsed mode", Sens. Actuat. A-Phys., 110 (2004) 269-275.

[5.4] A. Glaninger, A. Jachimowicz, F. Kohl, R. Chabicovsky, G. Urban, "Wide range semicoductor flow sensors", Sensor. Actuat. A-Phys., 85 (2000) 139-146.

[5.5] S-C. Roh, Y-M. Choi, S-Y. Kim, "Sensitivity enhancement of a silicon micromachined thermal flow sensor", Sensor. Actuat. A-Phys., 128 (2006) 1-6.

[5.6] T.H. Kim, S.J. Kim, "Development of a micro-thermal flow sensor with thin-film thermocouples", J. Micromech. Microeng., 16 (2006) 2502-2508.

[5.7] D. Randjelović, A. Petropoulos, G. Kaltsas, M. Stojanović, Z. Lazić, Z. Djurić, M. Matić, "Multipurpose MEMS thermal sensor based on thermopiles", Sens. Actuators A-Phys., 141 (2008) 404-413.

[5.8] Ch. Stamatopoulos, A. Petropoulos, D.S. Mathioulakis, G. Kaltsas, "Study of an integrated thermal sensor in different operational modes, under laminar, transitional and turbulent flow regimes", Exp. Therm. Fluid Sci., 32 (2008) 1687-1693.

[5.9] G.C.M. Meijer, A.W. van Herwaarden, "Thermal Sensors", Bristol, Institute of Physics Publishing (1994) chapter 4.

[5.10] T.S.J. Lammerink, N.R. Tas, G.J.M. Krijnen, M. Elwenpoek, "A new class of thermal flow sensors using $\Delta \mathrm{T}=0$ as a control signal", Proc. IEEE MEMS, Miyazaki, Japan (2000) 525-530.

[5.11] P. Bruschi, A. Diligente, D. Navarrini, M. Piotto, "A double heater integrated gas flow sensor with thermal feedback", Sensor. Actuat. A-Phys., 123-124 (2005) 210-215.

[5.12] M. Dijkstra, M.J. de Boer, J.W. Berenschot, T.S.J. Lammerink, R.J. Wiegerink, M. Elwenspoek, “A versatile surface channel concept for microfluidic applications”, J. Micromech. Microeng., 17 (2007) 1971-1977.

[5.13] H.K. Versteeg, W. Malalasekra, "An introduction to computational fluid dynamics, The finite volume method", Prentice Hall, Harlow, $1^{\text {st }}$ edn. (1995). 


\section{Low-Drift U-Shaped Thermopile Flow Sensor}

A thermal flow sensor has been realised consisting of a freely-suspended U-shaped microchannel. The structure is symmetrically heated by a heater at the top of the U-shape. The thermal imbalance caused by liquid flow is sensed by an integrated Al/poly-Si++ thermopile. The U-shape microchannel facilitates the integration of a large number of thermocouple leads, resulting in a highly-sensitive calorimetric flow sensor $\left(40 \mathrm{mV} / \mu \mathrm{l} \cdot \mathrm{min}^{-1}\right.$ at the largest heating power being $\left.2 \mathrm{~mW}\right)$. The heating power is controlled accurately by forcing a current, while measuring the voltage over the heater resistor. Influences of thermal gradients across the chip are minimised by the freely-suspended microchannel ends being fixed to the substrate over a small distance. The zero-offset of the thermopile at a uniform temperature can furthermore be exploited in a control system minimising temperature imbalance by liquid flow using additional heaters. This makes the flow sensor insensitive to heater resistor values and thermopile output characteristics. Accurate measurements up to about $300 \mathrm{nl} \cdot \mathrm{min}^{-1}$ water flow have been obtained applying a temperature-balancing control system. 6.1

\subsection{Introduction}

Flow sensors for accurate and reliable measurement of tiny fluid flow rates down to a few $\mathrm{nl} \cdot \mathrm{min}^{-1}$ have been developed $[6.2,6.3]$. So far, straight microchannels have been used (Chap. 5), applying thermopile read-out and power feedback [6.4], cancelling drift in the thermopile output voltage and heater resistors. With advanced control principles [6.4-6.7] significant improvement in drift performance can be obtained, but the sensor structure still suffers from ambient thermal gradients over the chip. An external temperature gradient will generated a thermopile output voltage and cannot be distinguished from an actual fluid flow through the device. In this chapter an attempt is made to realise a sensor structure that is also insensitive to external temperature gradients.

Figure 6.1 shows an existing commercially available mass flow sensor that is insensitive to thermal gradients due to a U-shaped tube structure. The sensor incorporates a single heater at the end of the U-shape creating a well defined thermal gradient to the base. A liquid flow results in a temperature imbalance over a thermopile suspended between the two legs of the U-shape structure. The sensor

6.1 This chapter is based on an article presented at IEEE Sensors conference [6.1]. 


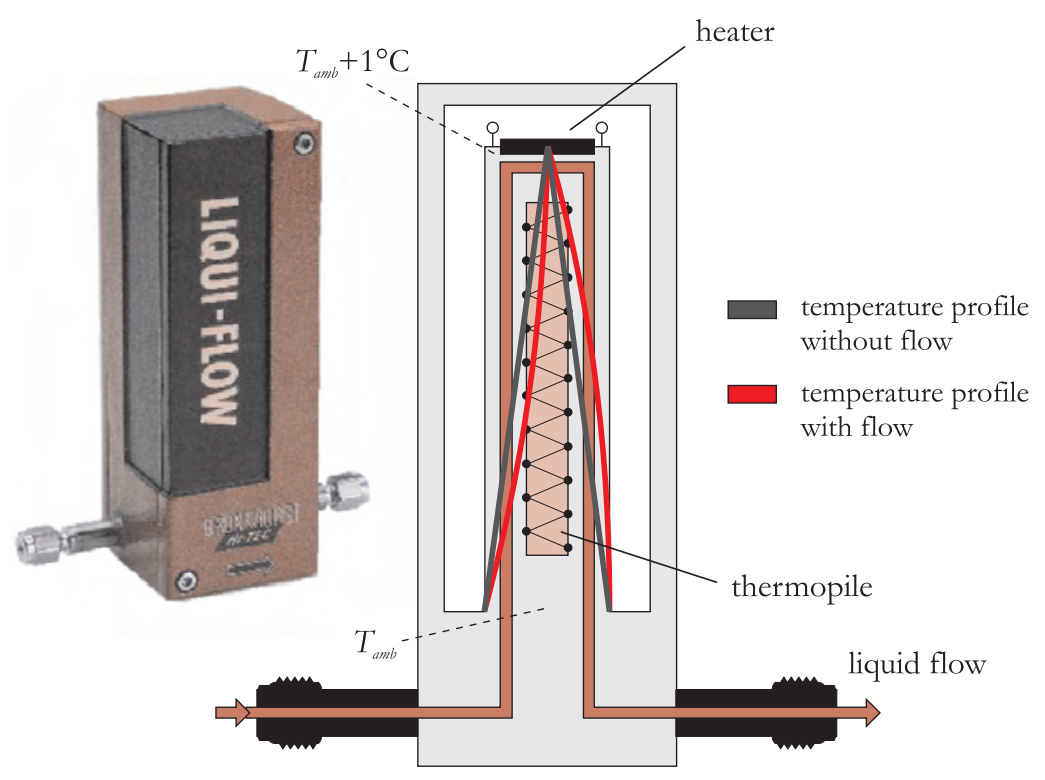

Fig. 6.1 The Liqui-Flow mass flow sensor from Bronkhorst Nederland B.V. [6.8].

proves to have an extremely stable zero with high sensitivity due to large number of thermocouple junctions that can be realised on the tube structure. In this chapter a similar sensor design is integrated on a silicon chip. The sensor design facilitates the integration of a large number of thermocouple junctions, while minimising the dependency on thermal gradients over the chip, because both ends of the U-shaped microchannel are fixed to the substrate over a small distance.

Figure 6.2 shows a representation of the freely-suspended U-shaped thermal flow sensor chip. Liquid flows through a microchannel embedded in the substrate. The freely-suspended $U$-shape microchannel is heated at the top by the gradient heater $R_{H}$, creating a thermal gradient towards the base of the freely-suspended structure. Without flow both sides of the U-shape are heated symmetrically. When flow is applied, one side of the U-shape decreases in temperature, while the other side increases in temperature, creating temperature imbalance similar to the commercial flow sensor (Fig. 6.1). A thermopile with junctions on either side of the U-shape measures the temperature difference and gives an output voltage $V_{\text {TC }}$ corresponding to the temperature imbalance. In this way, the sensor can be used as a calorimetric thermal flow sensor, with the voltage output of the thermopile giving a measure of the flow rate, while the power in the gradient heater can be controlled accurately using four-point measurement. 


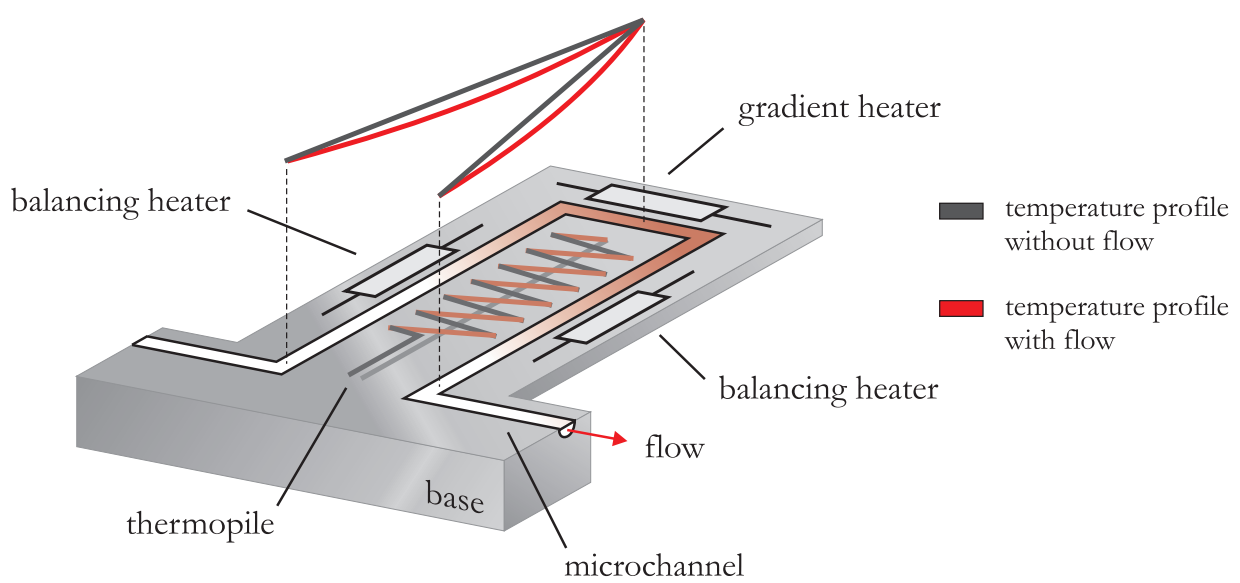

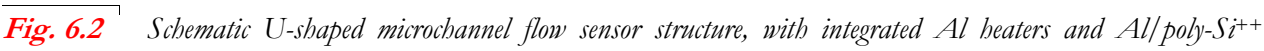
thermopile.

Additionally a control system can be used (Fig. 6.3), in order to obtain a flow sensor that is insensitive to resistance drift and non-linear thermopile characteristics $m_{T C}\left(\Delta T_{T C}\right)$. This requires the cancellation of the thermopile output voltage and thus the temperature imbalance between both sides of the U-shape, requiring two additional balancing heater resistors $R_{1}, R_{2}$. The power dissipated in both balancing heaters is also accurately controlled using four-point measurement and power control. The output voltage $\mathrm{V}_{\mathrm{TC}}$ of the thermopile is cancelled by controlling a power differ-

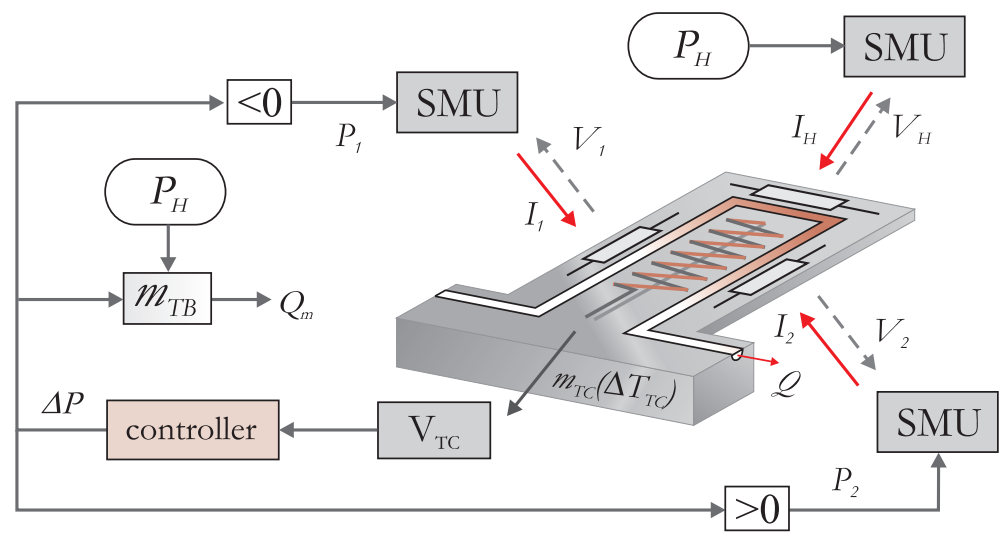

Fig. 6.3 Thermopile based power-feedback control system. 
ence $\Delta P$ between the two heater resistors, while dissipating a constant power $P_{H}$ in the gradient heater, according to the temperature-balancing anemometry principle [6.5]. The power difference $\Delta P$ is dissipated in one of the balancing heater resistors, with $R_{1}$ dissipating power when $\Delta P$ is negative and $R_{2}$ dissipating power when $\Delta P$ is positive, depending on the polarity of the thermopile.

As PI controller can be used to minimise the error $V_{T C}$, however as discussed in Chap. 5 a linear fit can be made, so that the correct $\Delta P$ is directly obtained using only two measurement points near $\Delta P$. This means the controller does not have parameters to adjust, cannot become instable and does not suffer from settling time. The temperature distribution changes on applying a fluid flow due to forced convection. This results in the relation $\Delta P / P_{H}$ being dependent on the flow rate $Q$. A measure of the flow rate $m_{T B}\left(\Delta P / P_{H}\right)$ can be obtained, with $\Delta P / P_{H}$ being linear for small flow rates.

\subsection{Sensor Fabrication}

The fabricated thermopile flow sensor incorporates mechanically-strong freely suspended surface microchannels for on-chip transport of fluid. Surface microchannels are fabricated without the requirement for a sacrificial layer and allow for the integration of sensor elements in close proximity to the fluid [6.9] as explained in Chap. 3.

Figure 6.4 gives a schematic overview of the process scheme for the fabrication of the U-shaped thermopile flow sensor. Surface microchannels are created by isotropic
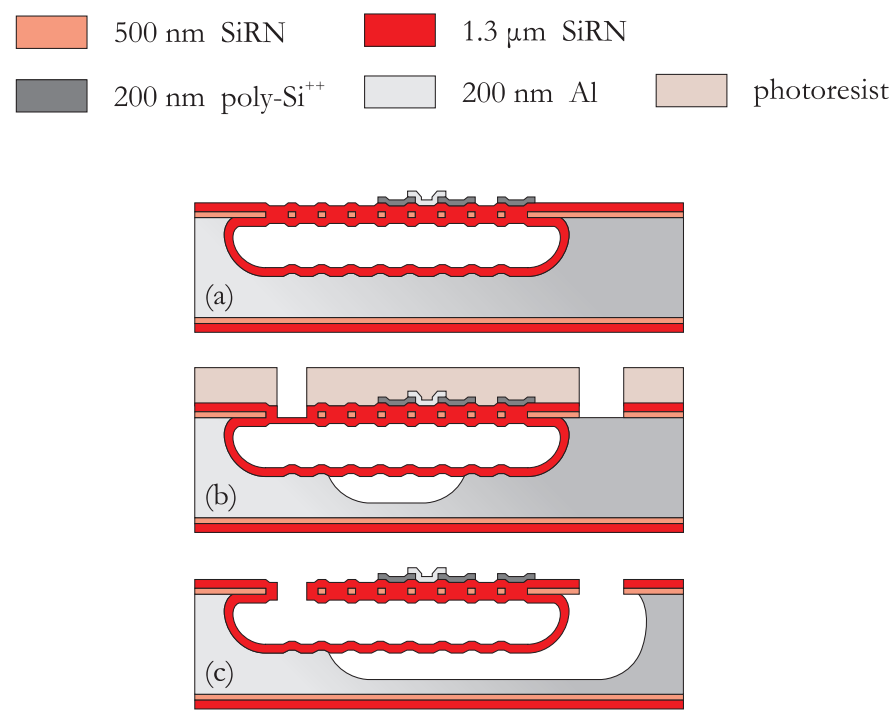

Fig. 6.4 Process scheme for the fabrication of the microchannel thermopile U-shaped microchannel flow sensor. 
dry etching, using high-density $\mathrm{SF}_{6}$ plasma with zero self-bias, through etch holes $2 \mu \mathrm{m}$ in width, in a low-stress $500 \mathrm{~nm}$ silicon-rich silicon-nitride (SiRN) layer. The etch holes and inner surfaces of the microchannels are conformally coated by a second low-stress LPCVD deposited $1.3 \mu \mathrm{m}$ SiRN layer, resulting in completely sealed microchannels, while leaving a planar substrate surface for the integration of an $\mathrm{Al} /$ poly-Si ${ }^{++}$thermopile and $\mathrm{Al}$ heater resistors.

The thermopile is created by LPCVD deposition and boron doping by solid source diffusion of a $200 \mathrm{~nm}$ poly-Si+ ${ }^{++}$layer and sputtering of a $200 \mathrm{~nm}$ Al layer (Fig. 6.4a). The surface microchannels are released by $\mathrm{SF}_{6}$ plasma etching, for thermal isolation from the heat-conducting substrate, with the photoresist mask protecting the sensor elements during the release (Fig. 6.4b). The photoresist is removed after fluidic entrance holes are etched through the SiRN layer, allowing for direct interfacing to the microchannels on the substrate surface.

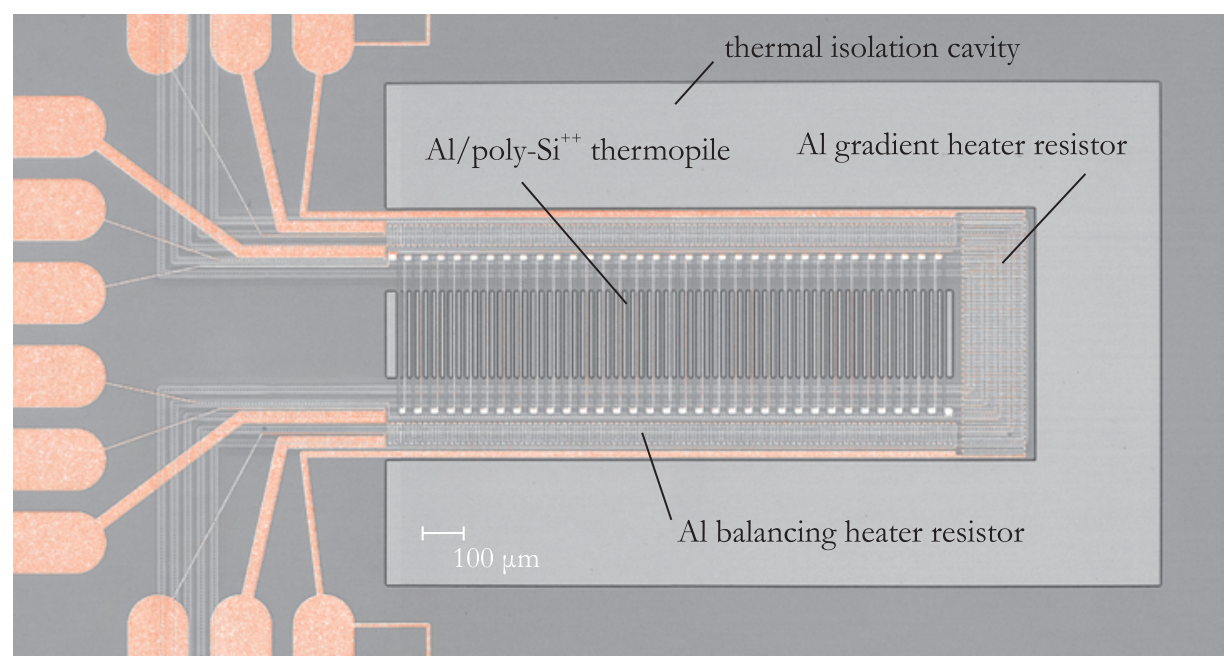

Fig. 6.5 U-shaped microchannel flow sensor just before release, showing the integrated Al heater resistors and Al/poly-Si+ thermocouples.

Figure 6.5 shows micrographs of the fabricated thermopile flow sensor. The sensor contains five parallel $15 \mu \mathrm{m}$ diameter microchannels, forming a $1.5 \mathrm{~mm}$ long U-shape freely suspended over a thermal isolation cavity etched in the silicon substrate. The thermal isolation cavity is etched to a depth of $230 \mu \mathrm{m}$, while the etch depth inside the U-shape is only $130 \mu \mathrm{m}$, due to the RIE lag around the thermocouple structures. The parallel microchannels have $200 \mu \mathrm{m}$ total width, which allows for the integration of the $\mathrm{Al}$ heater resistors and $\mathrm{Al} / \mathrm{poly}_{-} \mathrm{Si}^{++}$thermocouple leads. The thermopile has 34 junctions on either side of the U-shape structure. The $\mathrm{Al}$ heater resistors have four-point measurement contacts for accurate resistance measurements. 


\subsection{Experimental Results}

Fabricated $12.5 \times 12.5 \mathrm{~mm}$ thermopile flow sensor chips were characterised using a chip holder for electrical and fluidic connections (Fig. 6.6). The chip-holder contains O-rings for fluidic interfacing and twelve pogo-pins for electrical connection to the sensor chip. Water flow was applied by an elevation head up to a few meters (see Fig. 5.8), giving a stable flow rate. The flow is restricted by the hydraulic resistance of the microchannels and was determined by microbalance weighing.

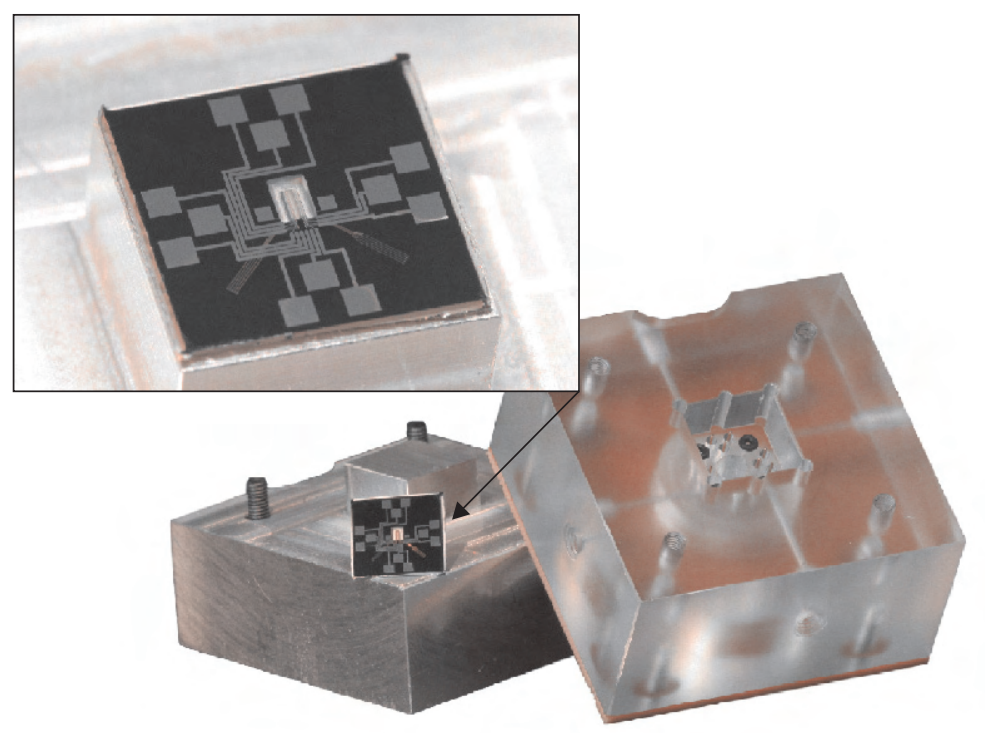

Fig. 6.6 Chip holder for fluidic and electrical interfacing with the sensor chip, measuring $12.5 \times 12.5 \mathrm{~mm}$. The inset shows an overview of the U-shaped microchannel flow sensor chip.

A source meter (Keithley 2420) was used to control the power in the gradient heater resistor. A two-channel source meter (Keithley 2602) was used to control the power on the balancing heater resistors. The thermopile output voltage was measured by a nano-volt meter (HP 34420A). The measurements were performed using MATLAB.

The flow sensor was first characterised as an open-loop calorimetric flow sensor applying different gradient heating powers $P_{H}$. The flow sensor shows a linear output response up to about $500 \mathrm{nl} \cdot \mathrm{min}^{-1}$ water flow rate (Fig. 6.7), with maximum sensitivity $\left(40 \mathrm{mV} / \mu \mathrm{l} \cdot \mathrm{min}^{-1}\right)$ at the largest $(2 \mathrm{~mW})$ heating power. At zero flow rate the thermal gradient is uniform along the U-shape structure, which gives no thermopile voltage and hence no output signal. As expected the sensor shows a well defined stable zerolevel. 


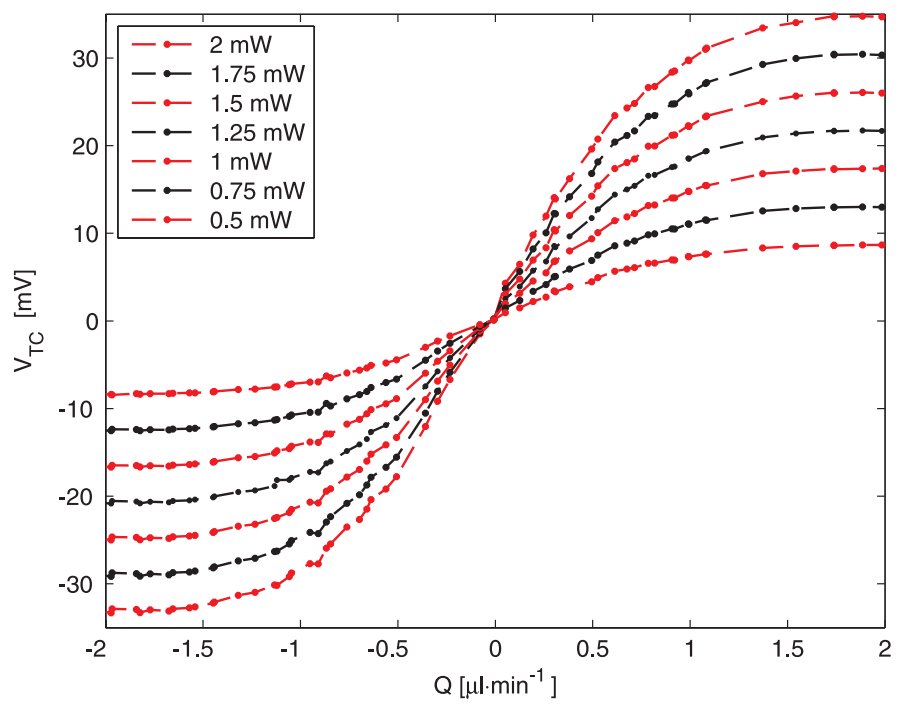

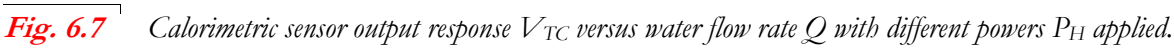

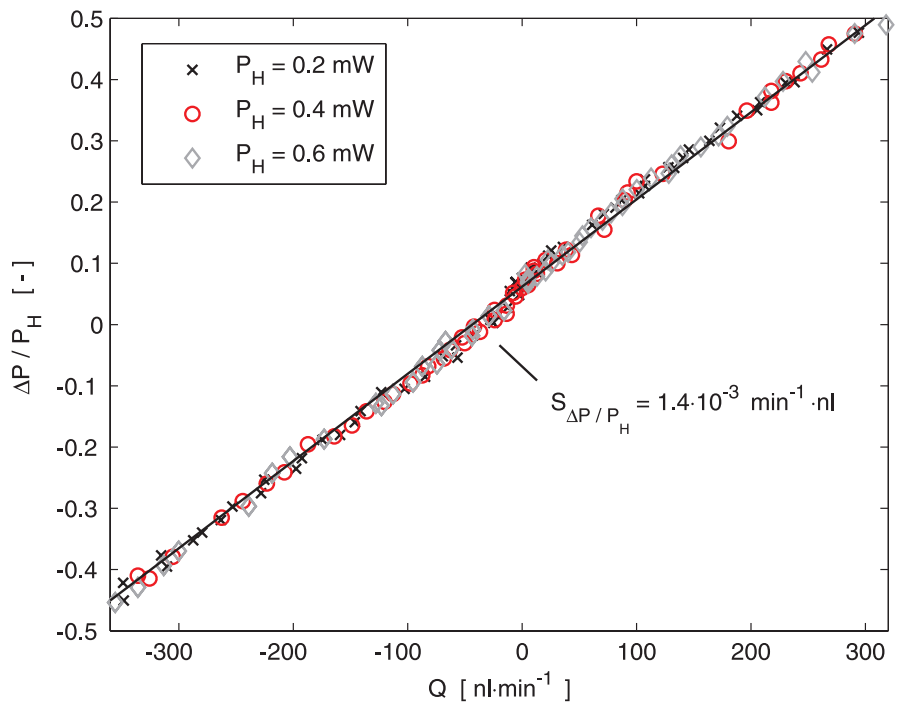

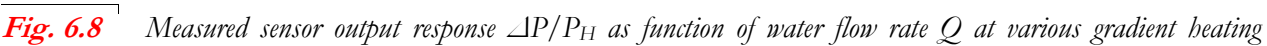
powers $P_{H}$. 
The sensor response to externally applied temperature gradients was characterised, using the setup described in Chap. 7. It appears that a thermal gradient in the direction of the thermopile leads still generates a thermopile voltage. This can be the result of the large thermal conductivity of the air surrounding the U-shaped microchannel. A temperature gradient perpendicular to the thermopile leads does not generate an output voltage.

The flow sensor was additionally characterised applying temperature balancing, by dissipating power in the balancing heater resistors, thus cancelling the temperature imbalance due to water flow. Figure 6.8 shows the measured $\Delta P / P_{H}$ sensor output having a linear response independent of gradient heating power $P_{H}$ with $1.4 \cdot 10^{-3} \mathrm{~min}^{-n l^{-1}}$ sensitivity.
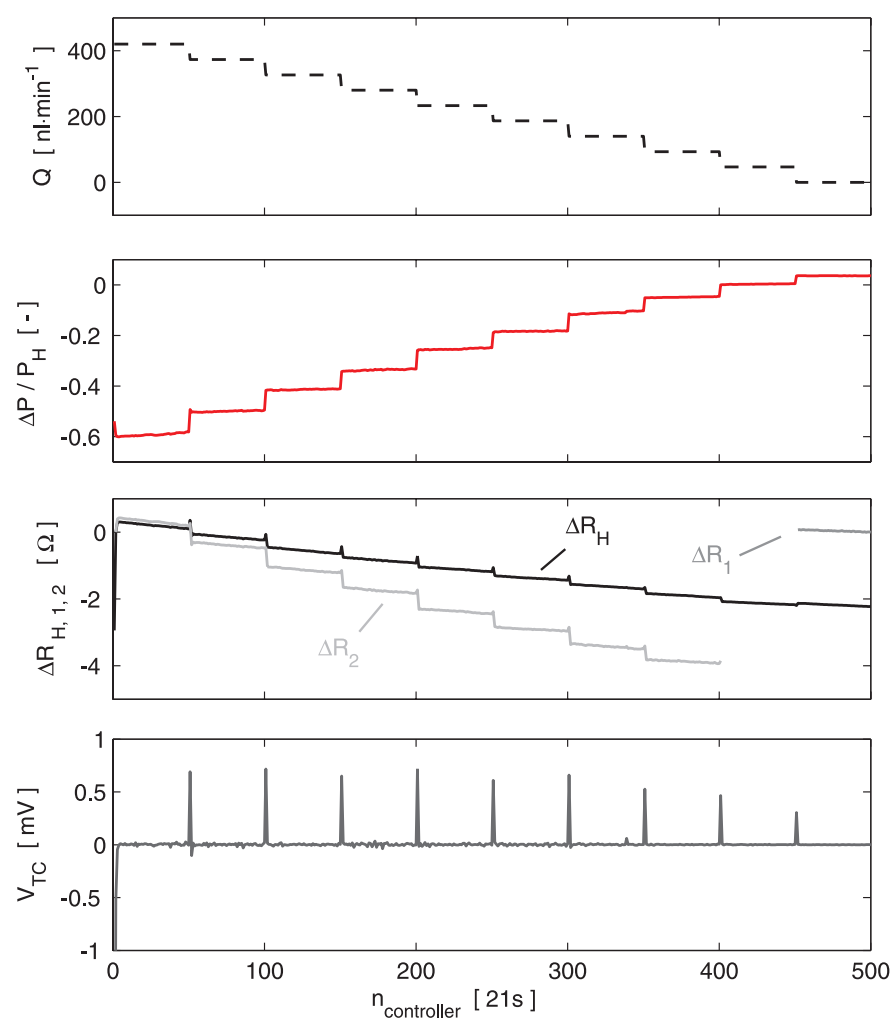

Fig. 6.9 Measured sensor control system response at changing water flow rate. The gradient heating power $P_{H}$ was kept constant at $0.4 \mathrm{~mW}$. 
Figure 6.9 shows the measured control system response on applying a changing water flow rate, with $0.4 \mathrm{~mW}$ gradient heating power. The sensor output $\Delta P / P_{H}$ gives a correct measure of the flow rate, while being independent of heater resistance values. Figure 6.9 shows that the balancing power $\Delta P$ is being dissipated in $R_{1}$ instead of $R_{2}$ after $\Delta P$ changes sign, while the thermopile voltage is correctly regulated to zero.

Instead of the PI controller used in Fig. 5.10 a linear fit was made using two measured thermopile voltages at different $\Delta P$. As a result the settling time of the sensor output in Fig. 6.9 is reduced to a few samples.

\subsection{Conclusions}

A thermal flow sensor consisting of a freely suspended U-shape microchannel with integrated $\mathrm{Al}$ heaters and an $\mathrm{Al} /$ poly-Si ${ }^{++}$thermopile has successfully been fabricated. A linear calorimetric sensor output was obtained for water flow rates up to about $500 \mathrm{nl} \cdot \mathrm{min}^{-1}$, with $40 \mathrm{mV} / \mu \mathrm{l} \cdot \mathrm{min}^{-1}$ sensitivity at the largest $(2 \mathrm{~mW})$ heating power. The heating power dissipated has been made independent of the heater resistance value, by using power control applying four-point measurement on the gradient heater resistor. Influence of thermal gradients across the chip has been minimised by the U-shape microchannel design, although a gradient parallel to the thermopile leads can still generates a small output voltage. The dependency on the non-linear characteristics of the thermopile has been minimised by using a control system, cancelling the temperature imbalance across the thermopile. A linear sensor response for water flow rates up to about $300 \mathrm{nl} \cdot \mathrm{min}^{-1}$, with $1.4 \cdot 10^{-3} \mathrm{~min}^{-\mathrm{nl}^{-1}}$ sensitivity, was measured using temperature balancing. The temperature-balancing control system operates correctly, without the possibility of instability and without settling time.

\section{References}

[6.1] M. Dijkstra, T.S.J. Lammerink, M.J. de Boer, J.W. Berenschot, R.J. Wiegerink, M.C. Elwenspoek, "Low-drift U-shaped thermopile flow sensor", Proc. IEEE Sensors, (2008).

[6.2] M. Dijkstra, M.J. de Boer, J.W. Berenschot, T.S.J. Lammerink, R.J. Wiegerink, M. Elwenspoek, "Miniaturized thermal flow sensor with planar integrated sensor structures on semicircular surface channels", Sensor. Actuat. A-Phys., 143 (2008) 1-6.

[6.3] Y. Mizuno, M. Liger, Y-C. Tai, "Nanofluidic flowmeter using carbon sensing element", Proc. IEEE MEMS, (2004) 322-325.

[6.4] M. Dijkstra, T.S.J. Lammerink, M.J. de Boer, J.W. Berenschot, R.J. Wiegerink, M. Elwenspoek, "Low-drift flow sensor with zero-offset thermopile-based power feedback", Proc. DTIP, (2008). 
[6.5] T.S.J. Lammerink, N.R. Tas, G.J.M. Krijnen, M. Elwenpoek, “A new class of thermal flow sensors using $\Delta \mathrm{T}=0$ as a control signal", Proc. IEEE MEMS, Miyazaki, Japan (2000) 525-530.

[6.6] P. Bruschi, A. Diligente, D. Navarrini, M. Piotto, "A double heater integrated gas flow sensor with thermal feedback", Sensor. Actuat. A-Phys., 123-124 (2005) 210-215.

[6.7] M. Domínguez, F.N. Masana, V. Jiménez, S. Bermejo, J. Amirola, J. Ballester, N. Fueyo, L.M. Castaner, "Low-cost thermal $\Sigma-\Delta$ air flow sensor", J. IEEE Sensors, 2 (2002).

[6.8] Bronkhorst Nederland B.V., "Liqui-flow - mass flow meters and controllers for liquids", Brochure (2002).

[6.9] M. Dijkstra, M.J. de Boer, J.W. Berenschot, T.S.J. Lammerink, R.J. Wiegerink, M. Elwenspoek, "A versatile surface channel concept for microfluidic applications", J. Micromech. Microeng., 17 (2007) 1971-1977. 


\section{Ambient Temperature-Gradient Compensated Low-Drift Thermopile Flow Sensor}

A highly-sensitive thermal flow sensor for liquid flow with nl-min ${ }^{-1}$ resolution has been
realised. The sensor consists of freely suspended silicon-rich silicon-nitride microchannels
with integrated Al heater resistors and Al/poly-Si+e thermopiles. The influence of
drift in the thin film metal resistors is effectively eliminated by using thermopiles
combined with an adequate measurement method, where the power in the beater
resistors is controlled, e.g. constant-power calorimetric method or temperature balancing
method. The special meandering layout of the microchannels and the placement of
thermopile junctions increases sensitivity by summing the thermopile voltages due to
convection by fluid flow, whereas the influence of ambient temperature gradients is
compensated for.7.1

\subsection{Introduction}

Fabricated micromechanical thermal flow sensors [7.2-7.5] have shown the capability of measuring down liquid flows down to $\mathrm{nl} \cdot \mathrm{min}^{-1}$ resolution. Important problems limiting the accuracy of these thermal flow sensors are: the drift in the electrical resistance of thin-film layers used for heating and temperature sensing, and the influence of external temperature gradients across the sensor chip [7.6]. Resistors used for temperature sensing can be avoided, by using thermopiles to measure a temperature difference, with zero-offset at a uniform temperature across the thermopile [7.7, 7.8]. Using thermopiles, the flow sensor can be made independent of resistance drift by applying power control on heater resistors. Additionally, a temperature-balancing feedback loop can be used to compensate for thermopile sensitivity drift [7.8].

This chapter describes a low-drift calorimetric flow sensor for liquid flows with $\mathrm{nl} \cdot \mathrm{min}^{-1}$ resolution. The flow sensor consists of freely suspended microchannels with integrated thermopiles for temperature sensing, with power control applied on heater resistors used. Furthermore, a special meandering microchannel layout is utilised to compensate for the influence of external temperature gradients across the sensor chip. The meandering layout eliminates the remaining sensitivity to temperature gradients in the U-shaped microchannel sensor [7.6] described in Chap. 6.

\footnotetext{
7.1 This chapter is based on an article presented at the IEEE MEMS 2009 conference [7.1].
} 


\subsection{Compensation Concept}

Ambient temperature-gradients are compensated using a meandering microchannel layout, in which a fluid flow $\mathcal{Q}$ passes in alternating direction through several freely suspended microchannels used for thermal flow sensing (Fig. 7.1). Thermopiles are connected in series and positioned on the freely-suspended microchannels in such a way that each thermopile measures a temperature difference $\Delta T$ between the upstream temperature $T_{\text {up }}$ and a downstream temperature $T_{\text {down }}$. Effectively the flow passes the same sensor configuration several times, while flowing through the meandering microchannel, giving a summation of multiple thermopile voltages due to convection by fluid flow.

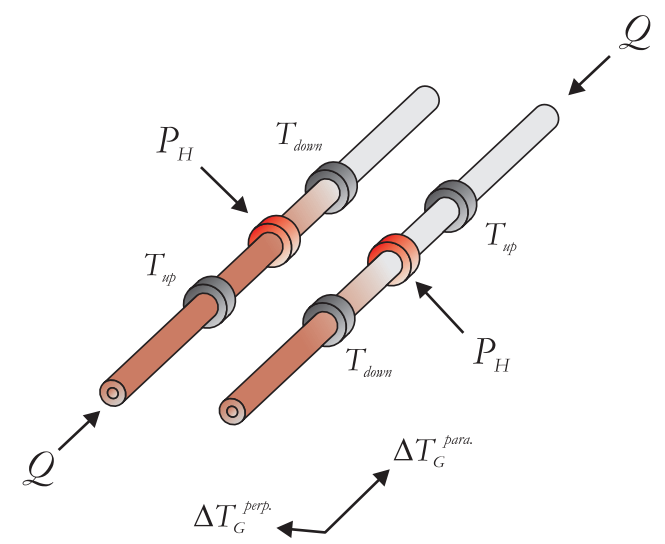

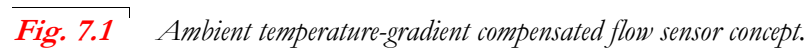

External temperature gradients $\Delta T_{G}$ of first order can be decomposed in two directions. A temperature gradient parallel to the microchannels $\Delta T_{G}$ para. changes $T_{u p}$ and $T_{\text {down }}$ in opposite ways on alternating microchannels, not influencing the total measured $\Delta T$. A gradient perpendicular to the microchannels $\Delta T_{G}$ perp. changes the offset temperature of each microchannel, not influencing the difference between $T_{u p}$ and $T_{\text {down }}$ on the same microchannel. First order temperature gradients can therefore be fully compensated. However, the heater resistors are slightly temperature dependent and therefore require control of the heating power $P_{H}$, in order for the compensation concept to work properly.

\subsection{Sensor Fabrication}

The fabricated thermopile flow sensor uses freely suspended surface microchannels for on-chip transport of fluid. The microchannels are fabricated without the requirement for sacrifical layer etching and allow for the integration of sensor ele- 
ments in close proximity to the fluid [7.9]. The technology has been applied in the fabrication of highly-sensitive flow sensors [7.5, 7.8, 7.10] and is explained in Chap 3 .

Figure 7.2 gives a schematic overview of the process scheme for the fabrication of the thermopile flow sensor. Surface microchannels are created by isotropic dry etching, using high-density $\mathrm{SF}_{6}$ plasma with zero self-bias, through etch holes $2 \mu \mathrm{m}$ in width, in a low-stress $500 \mathrm{~nm}$ silicon-rich silicon-nitride (SiRN) layer. The etch holes and inner surfaces of the microchannels are conformally coated by a second lowstress LPCVD deposited $1.3 \mu \mathrm{m}$ SiRN layer, resulting in completely sealed microchannels, while leaving a planar substrate surface for the integration of $\mathrm{Al} / \mathrm{poly}^{-\mathrm{Si}^{++}}$ thermopiles and $\mathrm{Al}$ heater resistors. The thermopiles are created by LPCVD deposition and boron doping, by solid source diffusion, of a $200 \mathrm{~nm}$ poly-Si ${ }^{++}$layer and sputtering of a $200 \mathrm{~nm}$ Al layer (Fig. 7.2a). The surface microchannels are released by $\mathrm{SF}_{6}$ plasma etching for thermal isolation from the heat-conducting substrate, with the photoresist mask protecting the sensor elements during the release (Fig. 7.2b). The photoresist is removed after fluidic entrance holes are etched through the SiRN layer (Fig. 7.2c).

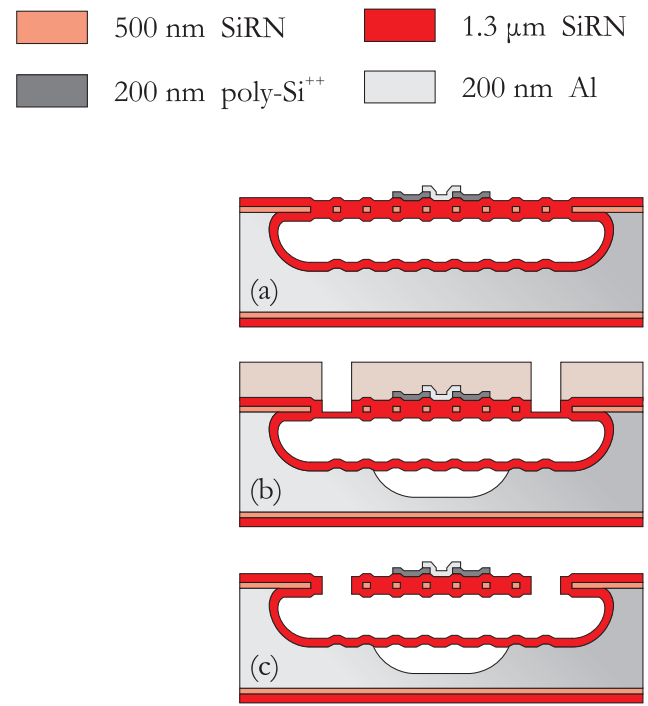

Fig. 7.2 Process scheme for the fabrication of the temperature-gradient compensated thermopile flow sensor.

Figure 7.3a shows a micrograph of the fabricated temperature-gradient compensated flow sensor. The sensor contains three parallel $20 \mu \mathrm{m}$ diameter microchannels, which cross a thermal-isolation cavity $(1.2 \times 2.0 \mathrm{~mm}, 250 \mu \mathrm{m}$ deep) several times in alternating flow directions. Heater resistors are centred on the freely-suspended microchannels and connected in series. The total resistance of the heater resistors is 


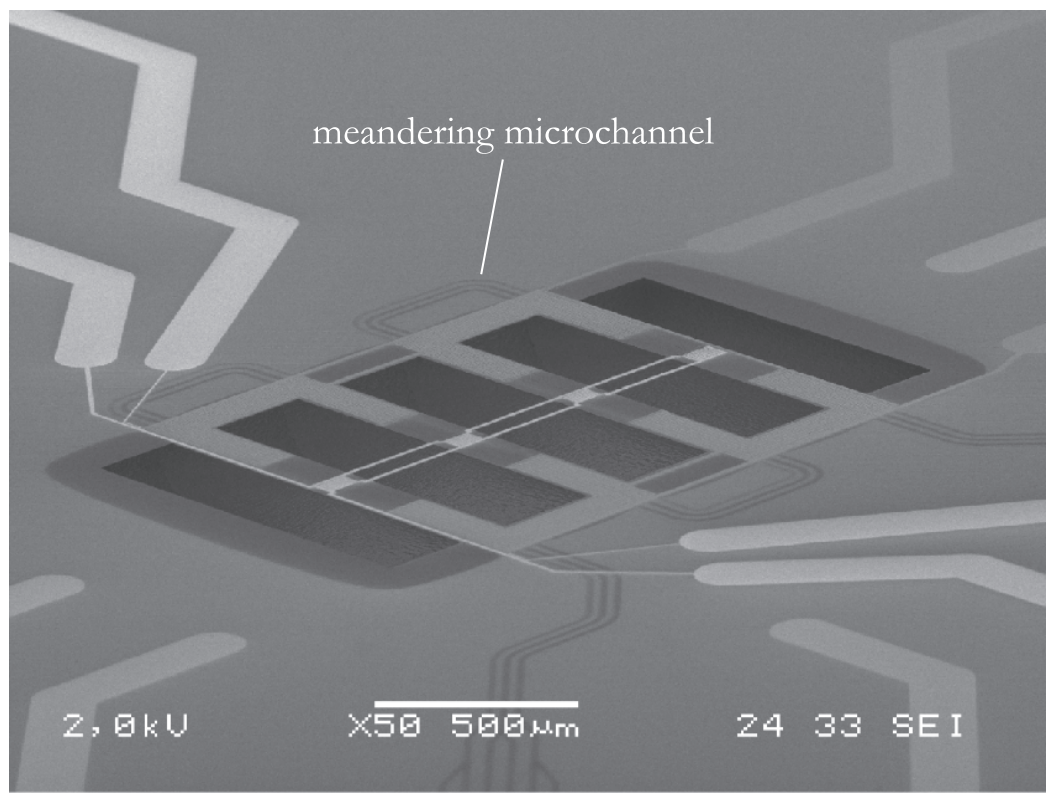

a)

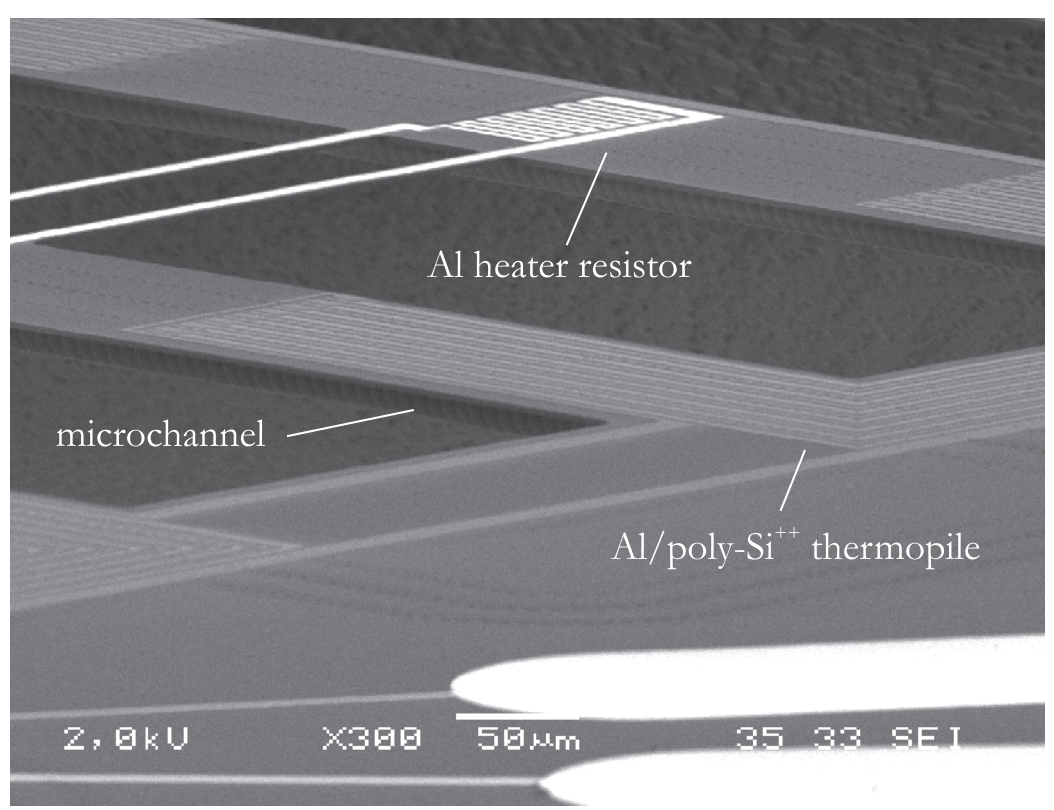

b)

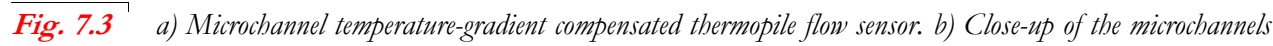
with integrated Al heater resistors and Al/poly-Si ${ }^{++}$thermopiles, suspended over a thermal isolation cavity etched in the substrate. 
determined accurately using four-point measurement. Of course for maximum accuracy all resisters should be measured and power controlled individually.

Figure 7.3a shows a close-up of the surface microchannels with integrated $\mathrm{Al} /$ poly-Si+ ${ }^{++}$thermopiles having eight junctions on either side, for measuring the down- and upstream temperature difference.

\subsection{Sensor Modelling}

A thermal model of the flow sensor was constructed in COMSOL Multiphysics [7.11]. The temperature field was solved including the thermal conduction in the flow sensor and surrounding air and forced convection by water flow through the microchannels. The flow inside the microchannels is approximated using a fixed velocity profile corresponding to a given flow rate, without affecting the accuracy of

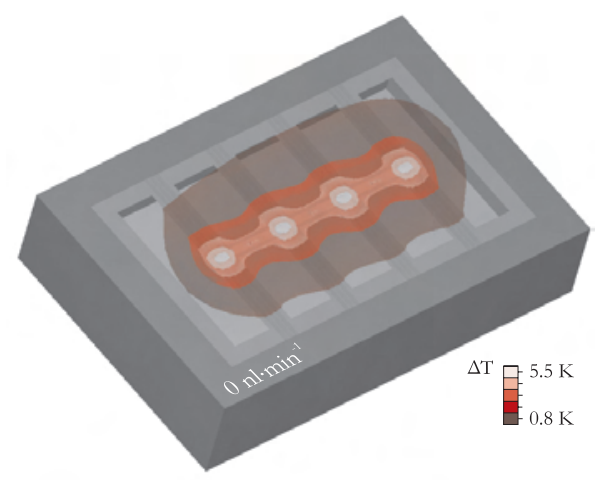

a)

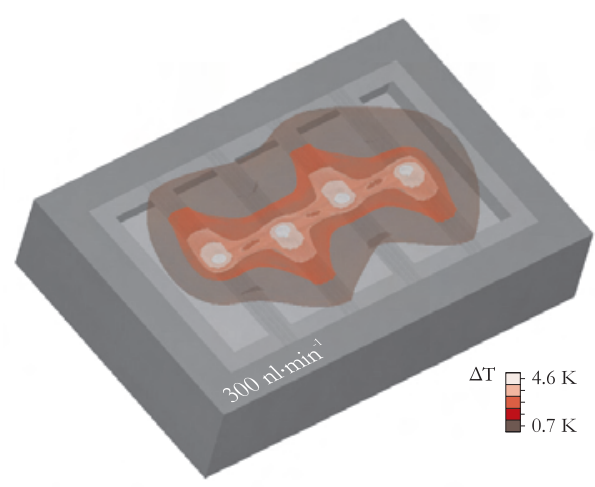

c)

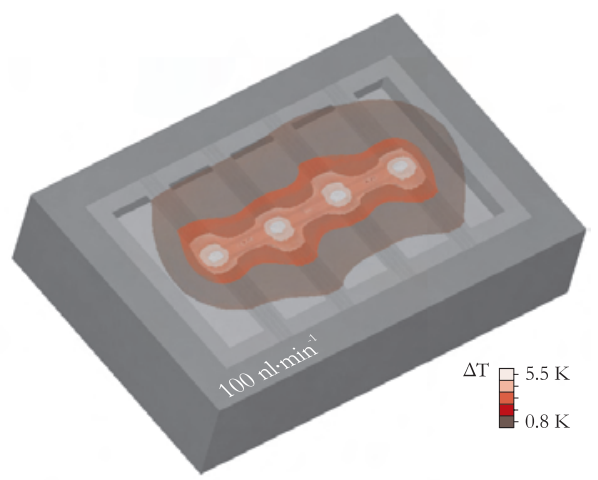

b)

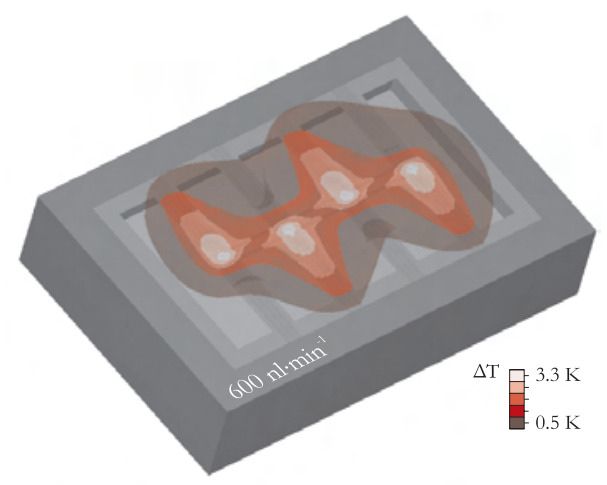

d)

Fig. 7.4 Thermal FEM model of the flow sensor showing the influence of convection by the alternating direction of various water flow rates through the microchannels at $0.6 \mathrm{~mW}$ total heating power. 
the thermal model. Additionally, deposited layers are modelled as highly conductive layers, including the thermopiles and heater resistors.

Figure 7.4 shows isothermal surfaces of the resulting temperature field at various water flow rates through the microchannels, with $0.6 \mathrm{~mW}$ total heating power applied. Clearly, the effect of forced convection by the alternating direction of the flow through the microchannels can be observed at this relatively high flow rate. The high thermal conduction of the $\mathrm{Al}$ leads connecting the heater resistors is also apparent, although there is no heat flow between adjacent microchannels for the temperature field by conduction alone.

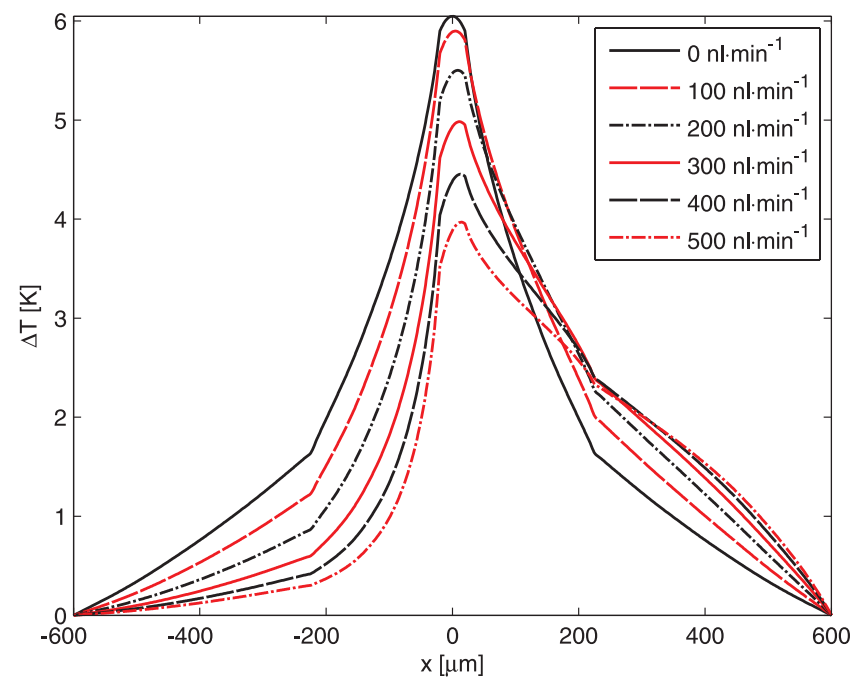

Fig. 7.5 Temperature profiles for various water flow rates in the positive $x$-direction along one of the microchannel segments suspended at $\pm 600 \mu \mathrm{m}$, with $0.6 \mathrm{~mW}$ total heating power applied.

Figure 7.5 shows temperature profiles along the microchannel at various water flow rates. A difference in the slope of the temperature profiles can be observed at $\pm 225 \mu \mathrm{m}$ from the centre, where the thermopile junctions are situated on the microchannel, with the thermopile leads causing much higher thermal conduction to the substrate.

\subsection{Experimental Results}

\subsubsection{Flow-Rate Sensitivity}

The fabricated thermopile flow sensors chips $(12.5 \times 12.5 \mathrm{~mm})$ have bondpads and microchannel entrance holes on fixed positions for self alignment with a chip holder (Fig. 7.6). The chip holder contains O-rings for fluidic interfacing and pogo-pins for 
electrical connections to the sensor chip. Water flow is applied by an elevation head $\Delta h$, giving a stable flow rate. The flow rate is calibrated by microbalance weighing, where evaporation from the balance, in the order of $100 \mathrm{nl} \cdot \mathrm{min}^{-1}$, has to be taken into consideration. A source measurement unit (Keithley 2420) was used for controlling a fixed total heating power $P_{H}$. The thermopile voltage $V_{T C}$ was obtained by a nano-volt meter (HP 34420A).

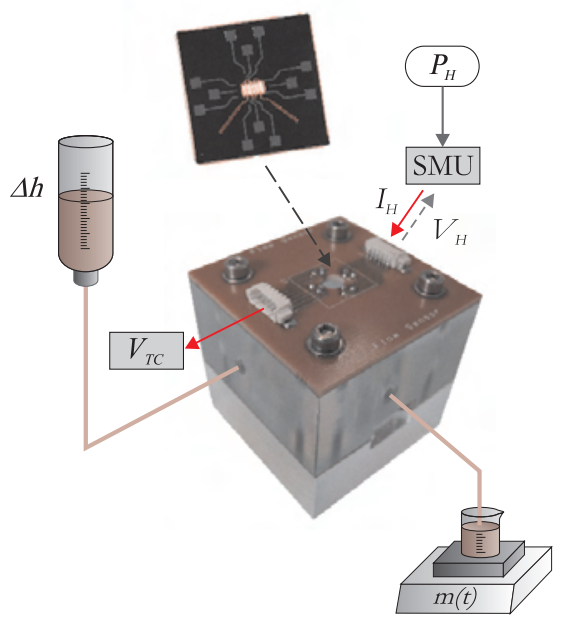

Fig. 7.6 Overview of the flow measurement setup, with the chip holder establishing fluidic and electrical connections.

Figure 7.7 shows the thermopile voltage $V_{T C}$ dependency on water flow rate $Q$ up to $550 \mathrm{nl} \cdot \mathrm{min}^{-1}$ at various applied heating powers. The sensor shows linear sensitivity up to flow rates of about $100 \mathrm{nl} \cdot \mathrm{min}^{-1}$. The heater temperature decreases at higher flow rates (Fig. 7.5) according to King's law, causing the sensor output to decrease. The dashed lines in Fig. 7.7 show fitted FEM results after scaling calculated temperature differences to measured voltages. Measurements show offsets in the thermopile voltage, which are dependent on the heating power and can be attributed to sensor and measurement asymmetries.

\subsubsection{Ambient Temperature-Gradient Sensitivity}

A temperature-gradient measurement setup was constructed in order to determine the influence of external temperature gradients on the thermopile voltage (Fig. 7.8). The setup consists of a $5 \mathrm{~mm}$ thick $\mathrm{Cu}$ plate, which is heated from one side by a resistor dissipating $25 \mathrm{~W}$, while the other side is connected to a large aluminium plate acting as heat sink, thus creating a well defined temperature gradient. The temperature gradient was determined to be approximately $2.8 \mathrm{~K} \cdot \mathrm{cm}-1$, measured using two Pt 100 


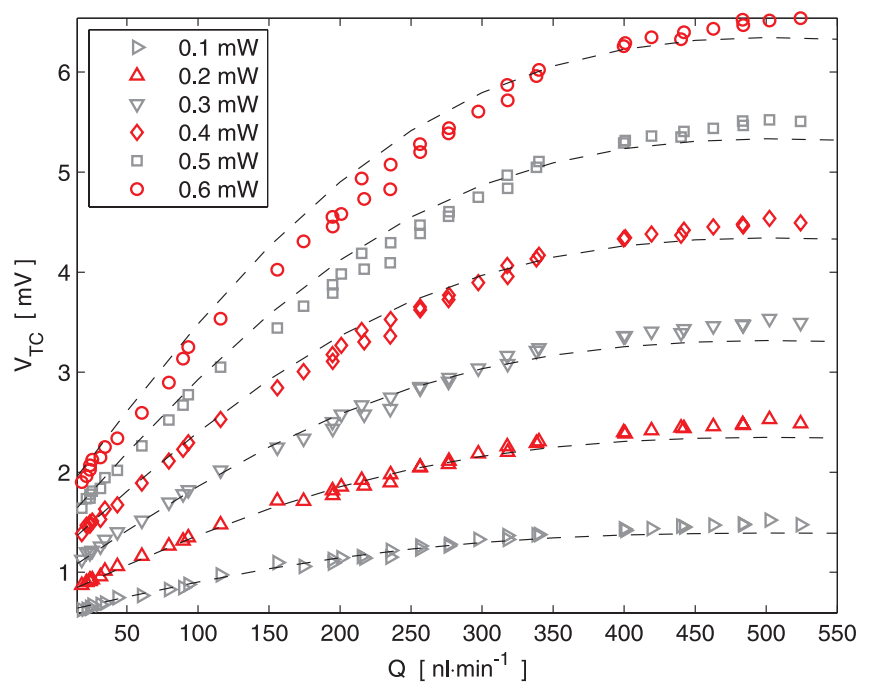

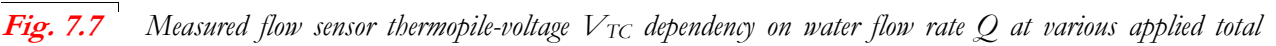
heating powers. Dashed lines show calculated thermopile temperature-differences from the FEM model scaled to the measurement points.

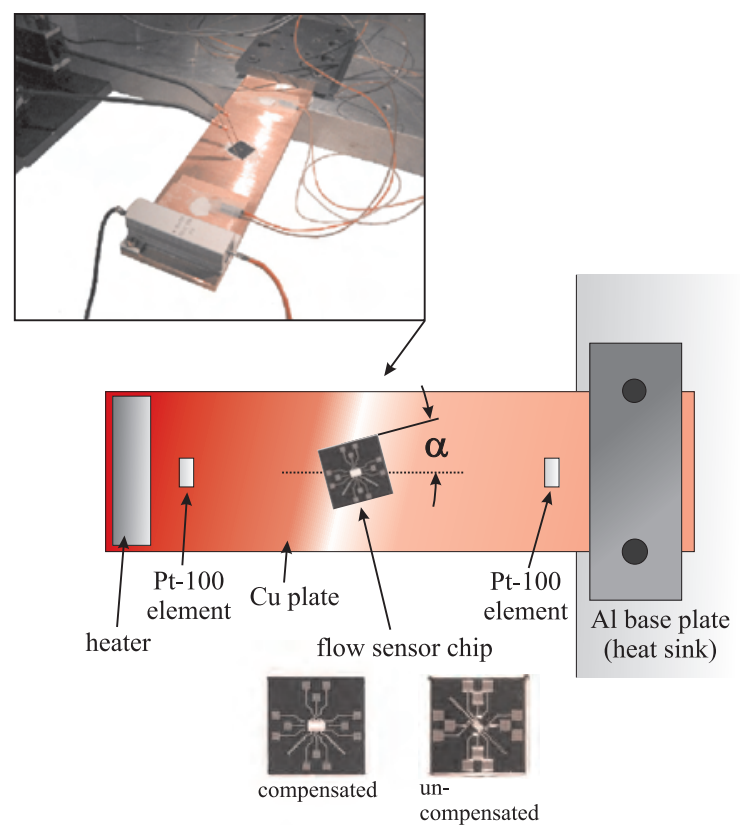

Fig. 7.8 Temperature-gradient measurement setup for inducing a well defined temperature gradient across the sensor chip. 


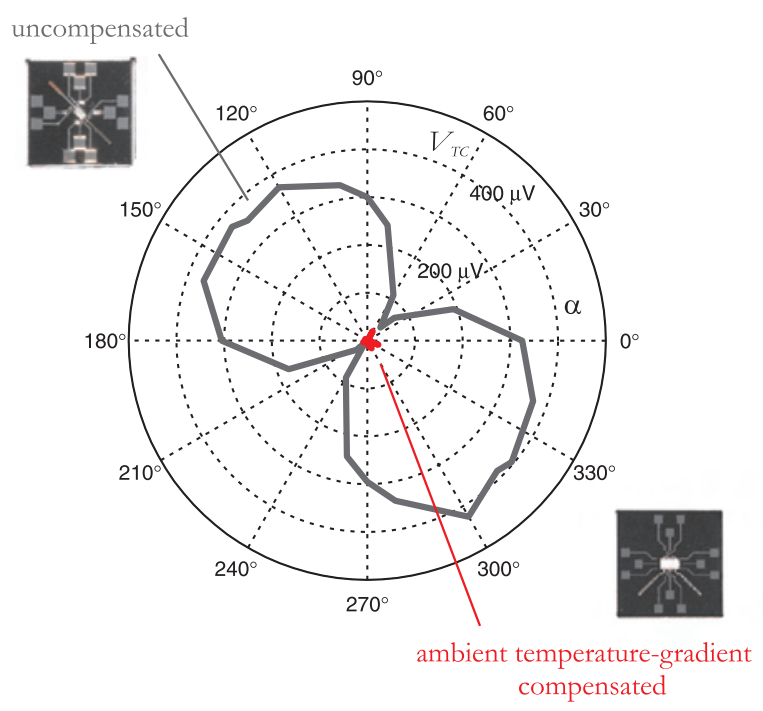

Fig. 7.9 External temperature-gradient sensitivity of the thermopile voltage $V_{\text {TC }}$ measured for a compensated sensor and a single microchannel uncompensated flow sensor.

elements placed $10 \mathrm{~cm}$ apart. The sensor chip is placed in the centre of the Cu plate and probes are used to measure the thermopile voltage using a nano-volt meter (HP 34420A). This allows the sensor chip to be rotated and the influence of the external temperature gradient to be measured at any angle.

An ambient temperature-gradient compensated flow sensor and an uncompensated single-microchannel flow sensor, for comparison, were measured, with both sensors having nearly equal amounts of thermopile junctions. The ambient temperaturegradient compensated flow sensor shows to be completely compensated for external temperature gradients (Fig. 7.9). The uncompensated flow sensor shows a figure-of-eight sensitivity pattern, because the flow sensor is only sensitive to temperature gradients parallel to the microchannel $\Delta T_{\text {para. }}$

\subsection{Conclusions}

A calorimetric flow sensor measuring liquid flow down to $\mathrm{nl} \cdot \mathrm{min}^{-1}$ resolution has been realised, using freely suspended microchannels for thermal isolation form the silicon substrate. The flow sensor shows a linear response for water flow up to about $100 \mathrm{nl} \cdot \mathrm{min}^{-1}$, which is accurately described by a thermal FEM model of the sensor.

It was demonstrated in Chap. 5 that drift in thin-film metal resistors can be effectively eliminated by using power control, in conjunction with thermopiles for temperature sensing. This in combination with the special meandering microchannel layout can effectively minimises drift in material properties an drift due to ambient temperature-gradients across the sensor chip. 
Compared to a single microchannel uncompensated flow sensor a significant improvement is obtained in sensor accuracy. The characteristic figure-of-eight to temperature gradients, which was observed even in the special U-shaped design (Chap. 6) has disappeared below the noise level.

\section{References}

[7.1] M. Dijkstra, T.S.J. Lammerink, M.J. de Boer, R.J. Wiegerink, M. Elwenspoek, "Ambient temperature-gradient compensated low-drift thermopile flow sensor", Proc. IEEE MEMS, (2009) 479-482.

[7.2] Y. Mizuno, M. Liger, Y-C. Tai, "Nanofluidic flowmeter using carbon sensing element", Proc. IEEE MEMS, (2004) 322-325.

[7.3] S. Wu, Q. Lin, Y. Yuen, Y-C. Tai, "MEMS flow sensors for nano-fluidic applications", Sensor. Actuat. A-Phys., 89 (2001) 152-158.

[7.4] H. Ernst, A. Jachimowicz, G.A. Urban, "High resolution flow characterization in BioMEMS”, Sensor. Actuator. A-Phys., 100 (2002) 54-62.

[7.5] M. Dijkstra, M.J. de Boer, J.W. Berenschot, T.S.J. Lammerink, R.J. Wiegerink, M. Elwenspoek, "Miniaturized thermal flow sensor with planar integrated sensor structures on semicircular surface channels", Sensor. Actuat. A-Phys., 143 (2008) 1-6.

[7.6] M. Dijkstra, T.S.J. Lammerink, M.J. de Boer, J.W. Berenschot, R.J. Wiegerink, M.C. Elwenspoek, "Low-drift U-shaped thermopile flow sensor", Proc. IEEE Sensors, (2008).

[7.7] P. Bruschi, A. Diligente, D. Navarrini, M. Piotto, “A double heater integrated gas flow sensor with thermal feedback", Sensor. Actuat. A-Phys., 123-124 (2005) 210-215.

[7.8] M. Dijkstra, T.S.J. Lammerink, M.J. de Boer, J.W. Berenschot, R.J. Wiegerink, M. Elwenspoek, "Low-drift flow sensor with zero-offset thermopile-based power Feedback", Proc. DTIP, (2008).

[7.9] M. Dijkstra, M.J. de Boer, J.W. Berenschot, T.S.J. Lammerink, R.J. Wiegerink, M. Elwenspoek, "A versatile surface channel concept for microfluidic applications", J. Micromech. Microeng., 17 (2007) 1971-1977.

[7.10] J. Haneveld, T.S.J. Lammerink, M. Dijkstra, H. Droogendijk, M.J. de Boer, R.J. Wiegerink, "Highly Sensitive Micro Coriolis Mass Flow Sensor", Proc. IEEE MEMS, (2008) 920-923.

[7.11] COMSOL Multiphysics AB - bttp:// www.comsol.com. 


\section{AC-Driven Temperature-Balancing Flow Sensor with Power Feedlback}

\footnotetext{
A microchannel thermal flow sensor with integrated Al resistors has successfully been fabricated. A control system has been implemented using a nearly offset-free powerfeedback signal applying heat waves, giving a sensor output independent of resistance values and thermo-electric offsets on interface wires. Four-point measurement and power control is used on heater resistors, which can therefore drift without influencing sensor output. The heat waves are detected at the sensor resistor using a lock-in amplifier and are mutually cancelled using a temperature-balancing controller. Offsets due to thermal gradients across the chip and sensor resistor drift are eliminated by the lock-in amplifier and therefore do not influence the sensor output signal. The flow sensor is capable of measuring water flow rates up to about 500 nl.min ${ }^{-1}$. Measurement results are in good agreement with a dynamic model of the flow sensor. Drift measurements show the compensation for resistance drift and thermal gradient in the sensor output signal. 8.1
}

\subsection{Introduction}

Increased miniaturisation of flow sensors has resulted in micro thermal flow sensors capable of measuring liquid flows down to a few nl. $\mathrm{min}^{-1}[8.2,8.3]$. These flow sensors require accurate measurement of very small flow induced temperature changes. Using a thermopile and advanced control techniques the influence of drift in resistance values and thermopile sensitivity can be largely eliminated. However, it is difficult to eliminate the influence of thermo-electric offset voltages, resistance drift and thermal-gradients completely.

In fact, non-homogeneous heating due to local variations in resistivity of the heater resistors cannot be prevented and will ultimately limit accuracy. Furthermore, irregularities in the thermopile material may result in thermopile voltages even when the average temperature difference between the junctions is zero. Therefore, a different approach [8.4] is investigated in this chapter, in which the heater resistors are heated by alternating currents, which may result in even greater accuracy using less complicated device structures. A disadvantage is a slower response time of the sensor,

8.1 This chapter is based on an article presented at the MME 2008 conference [8.1]. 


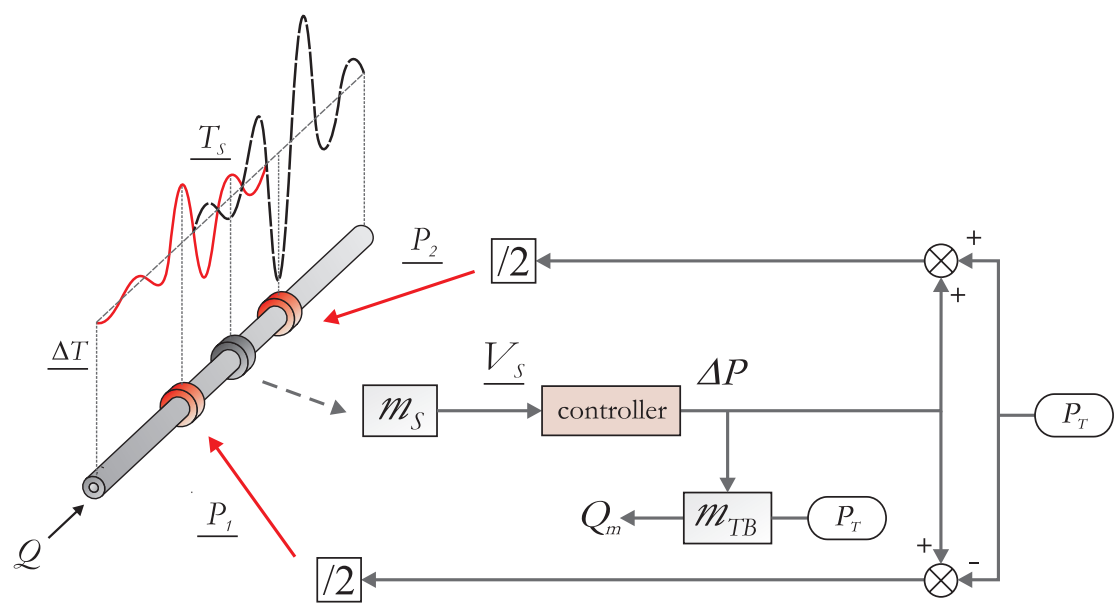

Fig. 8.1 Control system for the AC-driven temperature-balancing flow sensor.

since very low frequencies have to be used in order to obtain sufficiently large signal amplitudes.

Figure 8.1 shows a schematic drawing of a thermal flow sensor and control system that exploits sinusoidal heat waves, detected by a sensor resistor, to create a nearly offset-free power-feedback signal $\Delta P$ independent of sensor resistance value $R_{s}$. Two $180^{\circ}$ out-of-phase sinusoidal heat waves are generated by dissipating alternating currents in two resistors, up- and downstream of a central sensing resistors. The heating powers $P_{1}, P_{2}$ are controlled, with constant measurement of the resistance values of the heater resistors $R_{1}, R_{2}$, which can therefore drift without influencing sensor output. Thermo-electric offset voltages on interface wires are effectively eliminated by the lock-in amplifier. The lock-in amplifier also eliminates offset signals due to slowly varying thermal-gradients across the chip. The heat wave signals $\underline{T}_{S}$ detected by the sensor resistor $\underline{V}_{s}$ are mutually cancelled, minimising $\underline{V}_{s}$ by controlling $\Delta P$ between the two heater resistors, while keeping the total heating power $P_{T}$ dissipated constant [8.5-8.6]. The amplitude of $\underline{V}_{s}$ is controlled to a minimum by changing the power difference $\Delta P$. The exact amplitude of $\underline{V}_{s}$ is therefore not important and the sensor resistance is allowed to drift.

On applying a fluid flow the temperature distribution is changed by forced convection. This results in the relation $\Delta P / P_{T}$ being dependent on the flow rate $Q$. A measure of the flow rate $m_{T B}\left(\Delta P / P_{T}\right)$ can be obtained, with $\Delta P / P_{T}$ being linear for small flow rates. 


\subsection{Sensor Fabrication}

Thermal flow sensors have been fabricated using the surface channel technology [8.7], discussed in Chap. 3, for on-chip transport of fluid through mechanically strong freely suspended microchannels. The microchannels are fabricated below the wafer surface allowing for the integration of sensor elements in close proximity to the fluid.

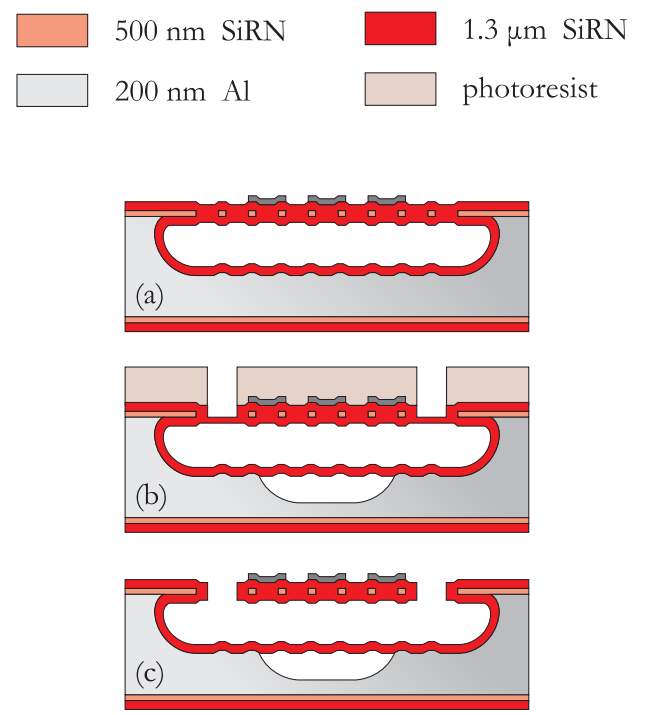

Fig. 8.2 Process scheme for the fabrication of the AC-driven temperature-balancing flow sensor.

Figure 8.2 shows the process scheme for the fabrication of the temperaturebalancing flow sensor. Surface microchannels are created by isotropic dry etching, using high-density $\mathrm{SF}_{6}$ plasma with zero self-bias, through etch holes $2 \mu \mathrm{m}$ in width, in a low-stress $500 \mathrm{~nm}$ silicon-rich silicon-nitride (SiRN) layer. The etch holes and inner surfaces of the microchannels are conformally coated by a second low-stress LPCVD deposited $1.3 \mu \mathrm{m}$ SiRN layer, resulting in completely sealed microchannels, while leaving a planar substrate surface for the integration of $\mathrm{Al}$ resistors (Fig. 8.2a). The surface microchannels are released by $\mathrm{SF}_{6}$ plasma etching, for thermal isolation from the heat-conducting substrate, with the photoresist mask protecting the sensor elements during the release (Fig. 8.2b). The photoresist is removed after fluidic entrance holes are etched through the SiRN layer, allowing for direct interfacing to the microchannels on the substrate surface. Figure 8.3 shows SEM micrographs of the fabricated microchannel thermal flow sensor. The sensor contains five parallel $20 \mu \mathrm{m}$ diameter microchannels, spanning a $1.2 \mathrm{~mm}$ thermal isolation cavity. Up- an down- 


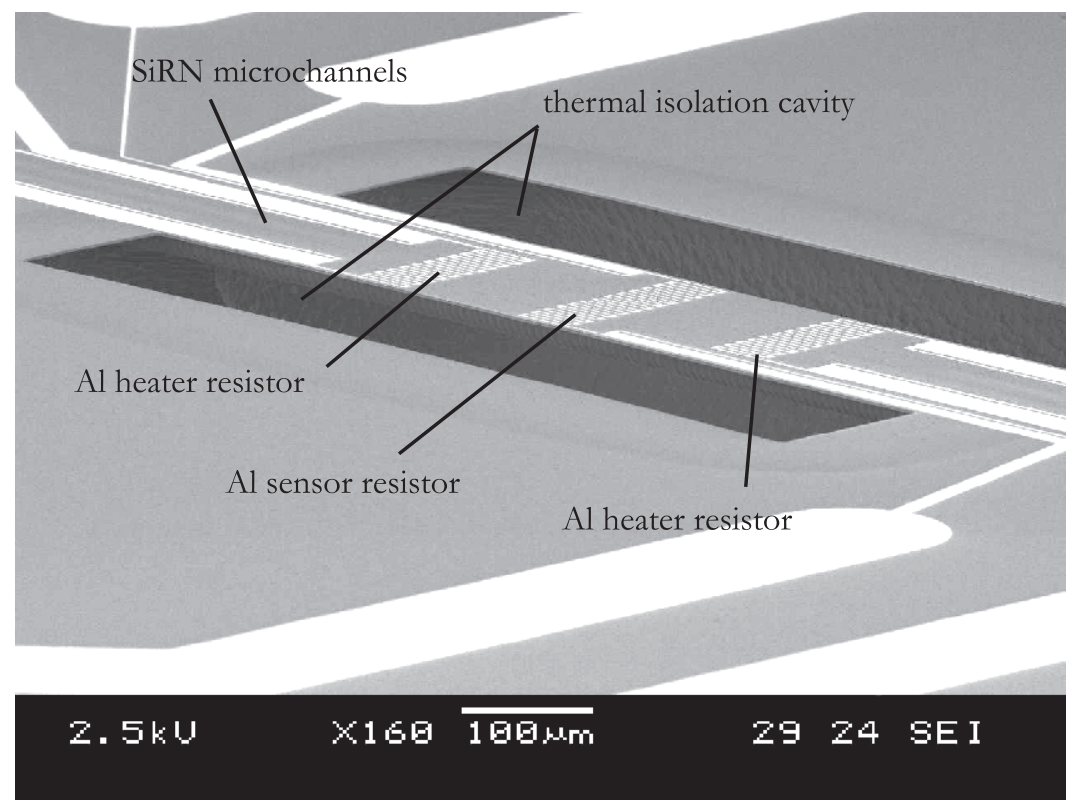

a)

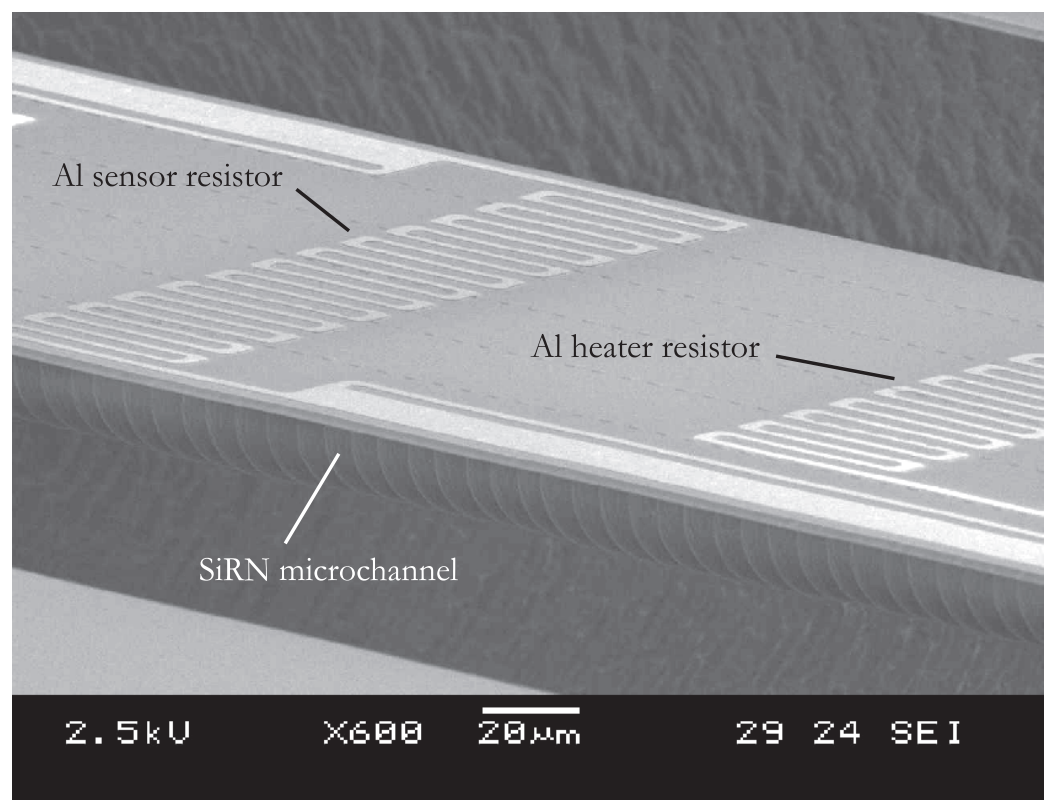

b)

Fig. 8.3 Micrographs of the temperature-balancing flow sensor, with a) sensor structure suspended over thermal isolation cavity and b) close-up of $A l$ resistors. 
stream $\mathrm{Al}$ heater resistors and an $\mathrm{Al}$ sensor resistor are integrated on top of the microchannels. The resistors have four-point contacts for accurate resistance measurements.

\subsection{Sensor Modelling}

A two-dimensional cylindrical finite volume method (FVM) model was constructed in MATLAB, solving the heat advection-diffusion equation for sinusoidal heat waves, with fluid flow along the $x$-direction. Using complex sparse-matrix calculations (8.1)

$$
\left(j 2 \omega \rho c+\rho c v_{x} \frac{\partial}{\partial x}-\nabla(\kappa \nabla)\right) T=P^{\prime \prime \prime}
$$

can be solved on a cylindrical mesh of the sensor, resulting in a heat capacity matrix $\mathbf{B}$, a convection matrix $\mathbf{G}_{\mathbf{Q}}$ and a conduction matrix $\mathbf{G}_{\mathbf{\kappa}}$, which can be solved directly using the Gauss-Seidel method on (8.2).

$$
\begin{array}{r}
\left(j 2 \omega \cdot \boldsymbol{B}+Q \cdot \boldsymbol{G}_{\boldsymbol{Q}}-\boldsymbol{G}_{\boldsymbol{\kappa}}\right) \overrightarrow{\vec{T}}_{e}=\underline{\vec{P}}_{e} \\
\underline{\boldsymbol{Y}} \underline{\vec{T}}_{e}=\underline{\vec{P}}_{e}
\end{array}
$$

The column vectors $\underline{T}_{e}$ and $\underline{\vec{P}}_{e}$ contain respectively the resulting complex temperature and complex power input for each element in the FVM mesh. The $180^{\circ}$ out-of-phase sinusoidal heat waves are given as two complex vector at the heater positions, with amplitude determined by temperature-balancing.

Figure 8.4 shows calculated temperature fields, represented by the amplitude and phase of the complex field. Fig. 8.4a shows the temperature field with no flow applied. Two heat waves are generated at the location of the heater resistors, having equal amplitude at zero flow, where the phase plot shows that both heat waves are actuated $180^{\circ}$ out of phase. In Fig. $8.4 \mathrm{~b} 500 \mathrm{nl} \cdot \mathrm{min}^{-1}$ water flow is applied, where the downstream heater dissipates more than the upstream heater maintaining a minimum in the amplitude of the temperature at the sensor resistor. A small asymmetry can be seen in the phase plot of Fig. 8.4b, due to forced convection in the microchannel.

The heat waves at $5 \mathrm{~Hz}$ extend only within one period between the heater resistor and sensor resistor. Figure 8.4c shows the temperature field with no flow applied at $1 \mathrm{kHz}$. The heat wave has more than one period inside the sensor structure, while the amplitude of the heat wave has decreased significantly. The heat capacity of the fluid inside the microchannel results in the fluid retaining most of the dissipated heat. 


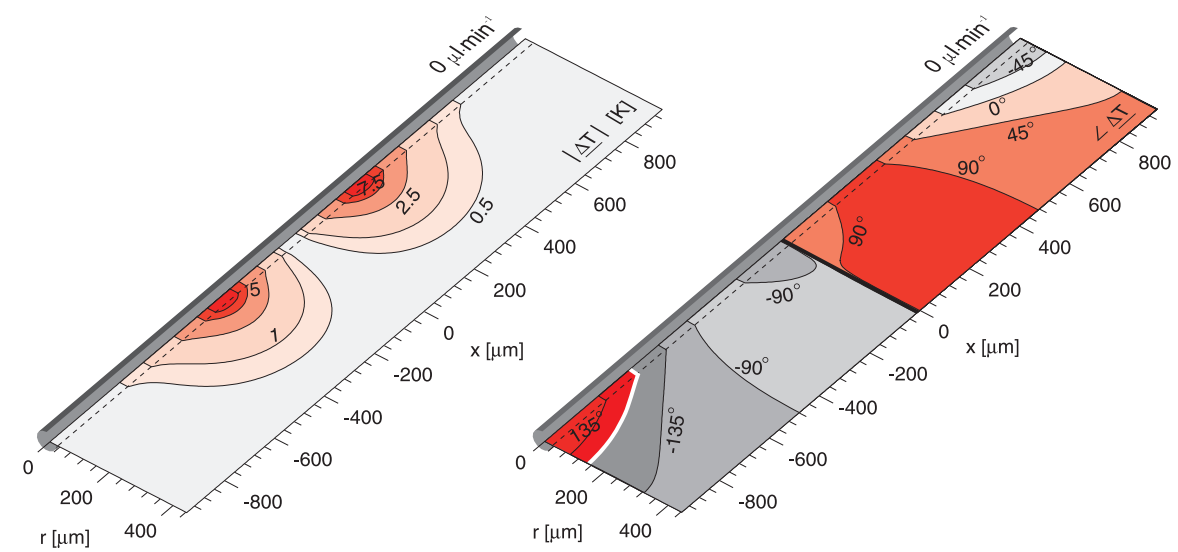

a)

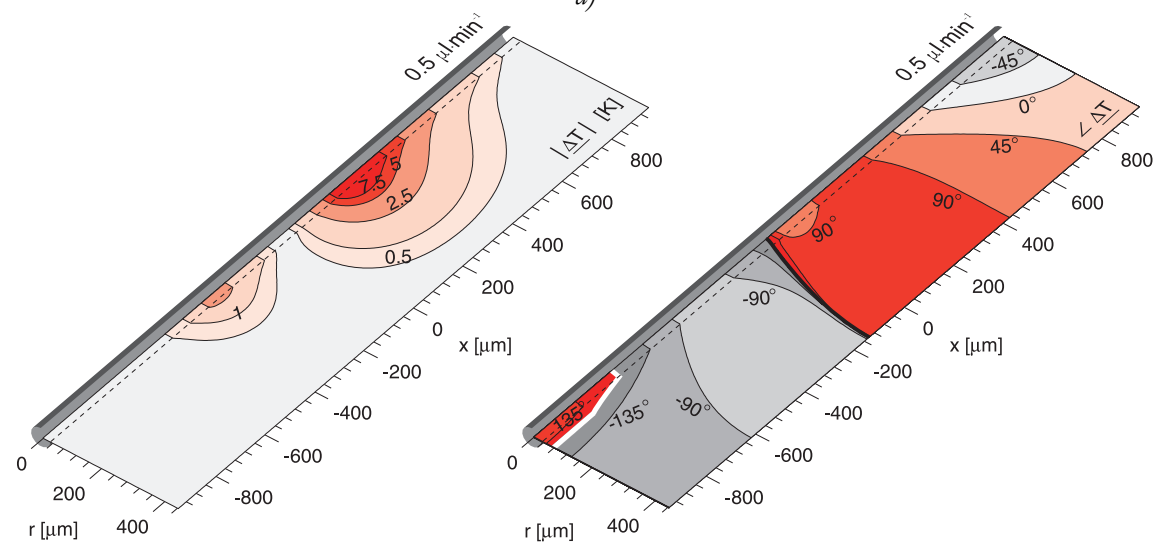

b)

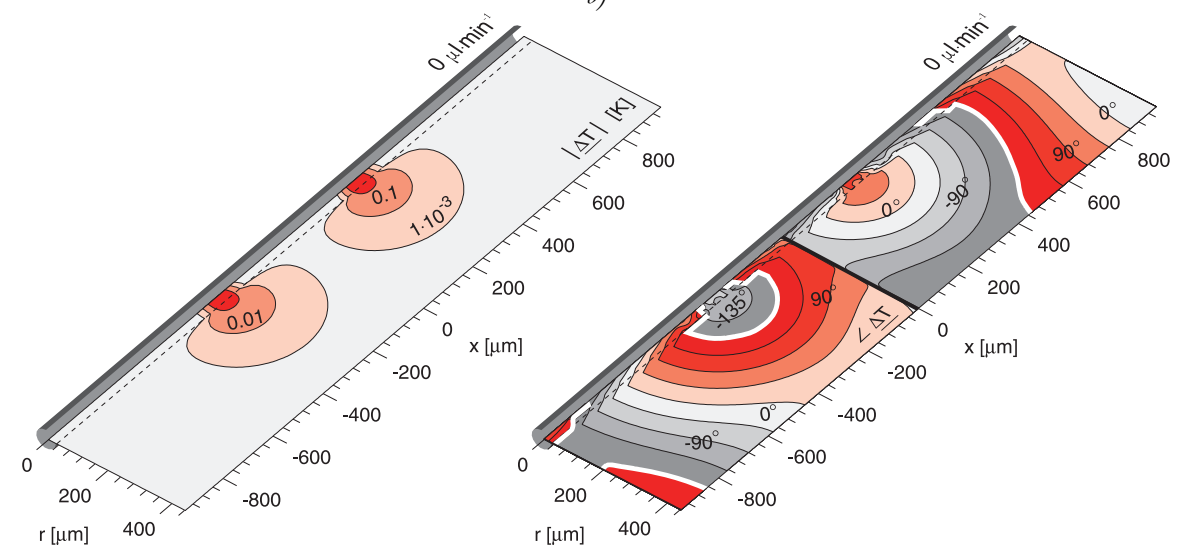

c)

Fig. 8.4 Calculated temperature amplitude (left) and phase (right) fields with $3 \mathrm{~mW}$ total power $P_{T}$ at a) $5 \mathrm{~Hz}$ without flow, b) $5 \mathrm{~Hz}$ with $500 \mathrm{nl} \cdot \mathrm{min}^{-1}$ water flow and c) $1 \mathrm{kHz}$ without flow. 

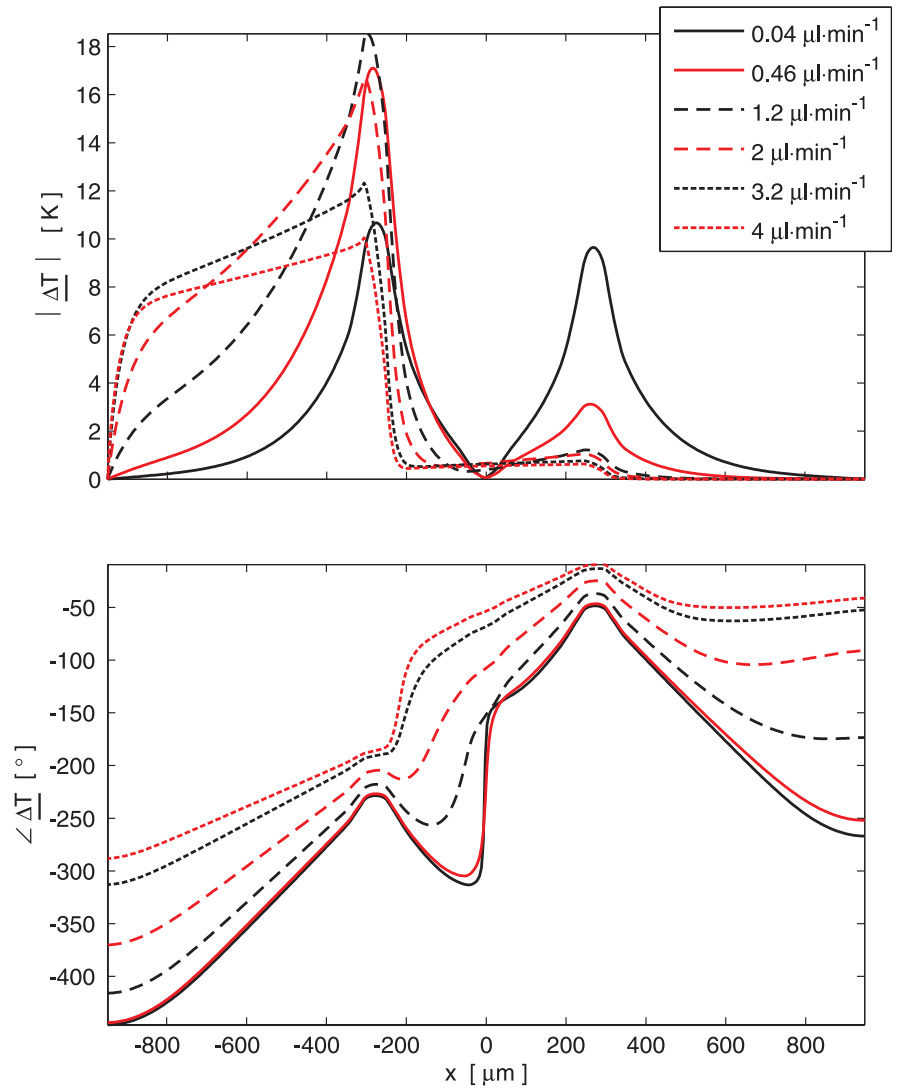

Fig. 8.5 Calculated temperature amplitude and phase profiles along the microchannel at $5 \mathrm{~Hz}$ with $3 \mathrm{~mW}$ total beating power $P_{T}$ and various water flow-rates $Q$ in the negative $x$-direction.

Figure 8.5 shows resulting temperature profiles along the microchannel from FVM model calculations. The temperature-balancing of the heat waves, minimising the amplitude at the sensor resistor, was simulated for different flow rates $Q$. It can be observed from Fig. 8.5 that the amplitude in temperature at the sensor resistor can be made zero for flow rates up to $0.46 \mu \mathrm{l} \cdot \mathrm{min}^{-1}$. At flow rates of $1.2 \mu \mathrm{l} \cdot \mathrm{min}^{-1}$ and higher a minimum can be obtained at the sensor resistor but the temperature amplitude cannot be made completely zero, due to the large forced convection from the upstream heater.

\subsection{Experimental Results}

Fabricated thermal flow sensor chips were characterised using a chip holder for electrical and fluidic connections (Fig. 8.6). Water flow was applied by adjusting an 


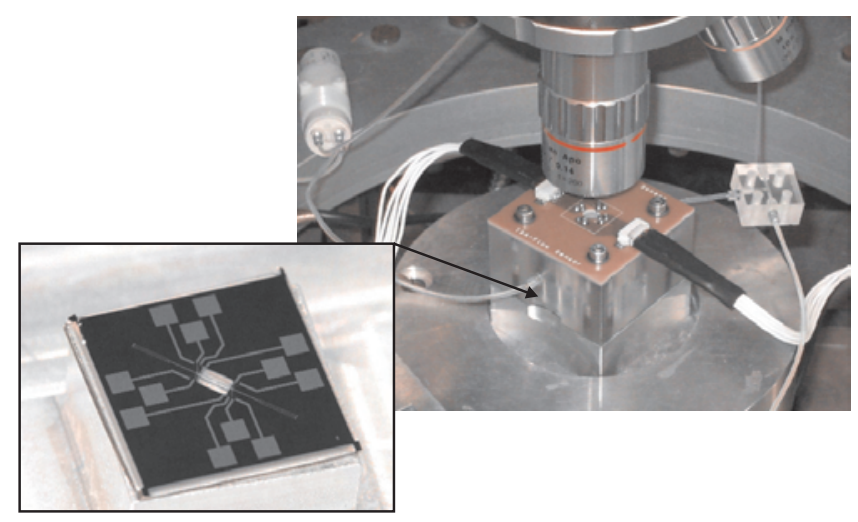

Fig. 8.6 Electrical and fluidic interconnection to the sensor chip. Inset shows a photograph of the temperature-balancing flow sensor chip.
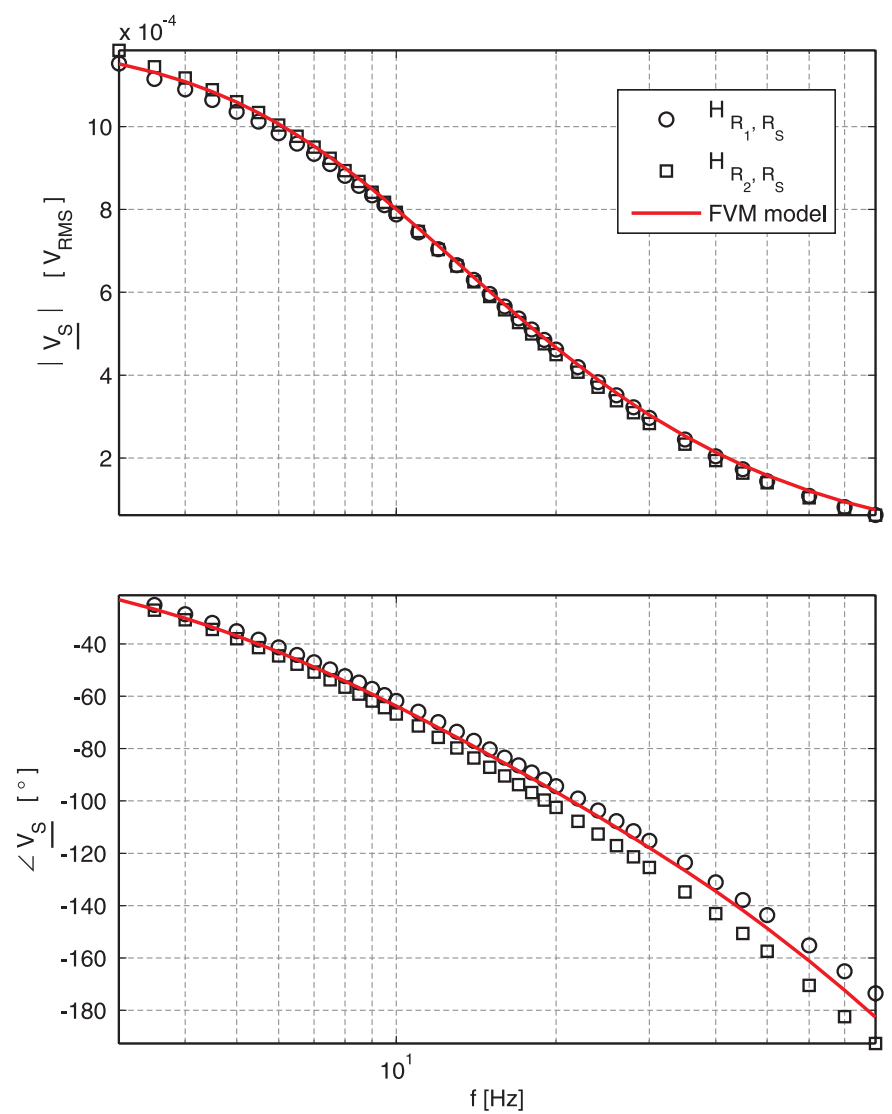

Fig. 8.7 Frequency response of each heater resistor $R_{1}, R_{2}$ to the sensor resistor $R_{S}$ at $0.4 m W$ power $P_{T}$ compared to FVM model calculations. 
elevation head, while the actual flow rate was calibrated by microbalance weighing. The measurements were controlled by MATLAB. The heater resistors were heated by applying an alternating current (HP 3245A). The heating power was controlled accurately by reading the voltage drop over the heater resistors using multimeters (HP 34401A) and by adjusting the AC amplitude accordingly. A small direct current was passed through the sensor resistor (Keithley 237), while the $2 \omega$ component in the voltage drop over the sensor resistor was measured by a DSP lock-in amplifier (SRS 830).

Figure 8.7 shows the open-loop frequency response, simulated, and measured for each heater resistor to the sensor resistor, without flow. The amplitude is proportional to $\sqrt{1 / f}$, due to the frequency dependency on the thermal penetration depth of the sinusoidal heat wave. A small phase difference between both heaters can be observed
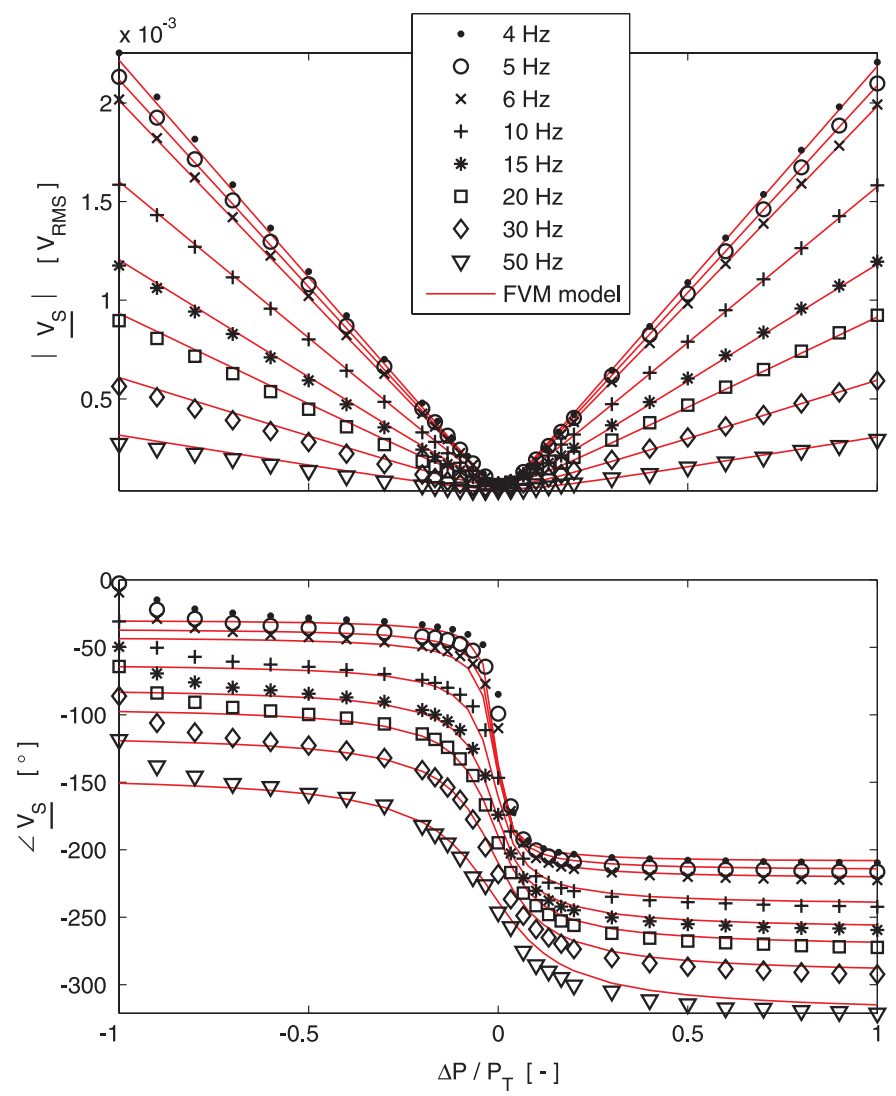

Fig. 8.8 Open-loop sensor resistor response on power difference $\triangle P$ without flow at various frequencies with $0.8 \mathrm{~mW}$ total heating power $P_{T}$ applied compared to FVM model calculations. 
in Fig. 8.7 for higher frequencies, indicating a small asymmetry in the flow sensor.

Figure 8.8 shows the sensor response on the power difference $\Delta P$ between both heaters. Minimum amplitude for $\underline{V}_{s}$, independent of frequency, is obtained when both heaters dissipate equal amount of power. The determination of the minimum in amplitude is independent of the sensor resistance value $R_{S}$ and is theoretically offset and drift free. However, asymmetries in the sensor structure can still give rise to an offset in $\Delta P$, which shows to be more apparent in close-loop operation. The decrease in slope of the phase of $\underline{V}_{s}$ near zero $\Delta P / P_{T}$ at higher frequencies (Fig. 8.8) is caused by the sensor asymmetry measured in Fig. 8.8. The FVM model results only show this decrease in slope when the phase difference from Fig. 8.7 is taken in consideration.

The position of the minimum in the amplitude of $\underline{V}_{s}$ changes with flow rate applied. Figure 8.9 shows measured and simulated open-loop response on $\Delta P$ to var-
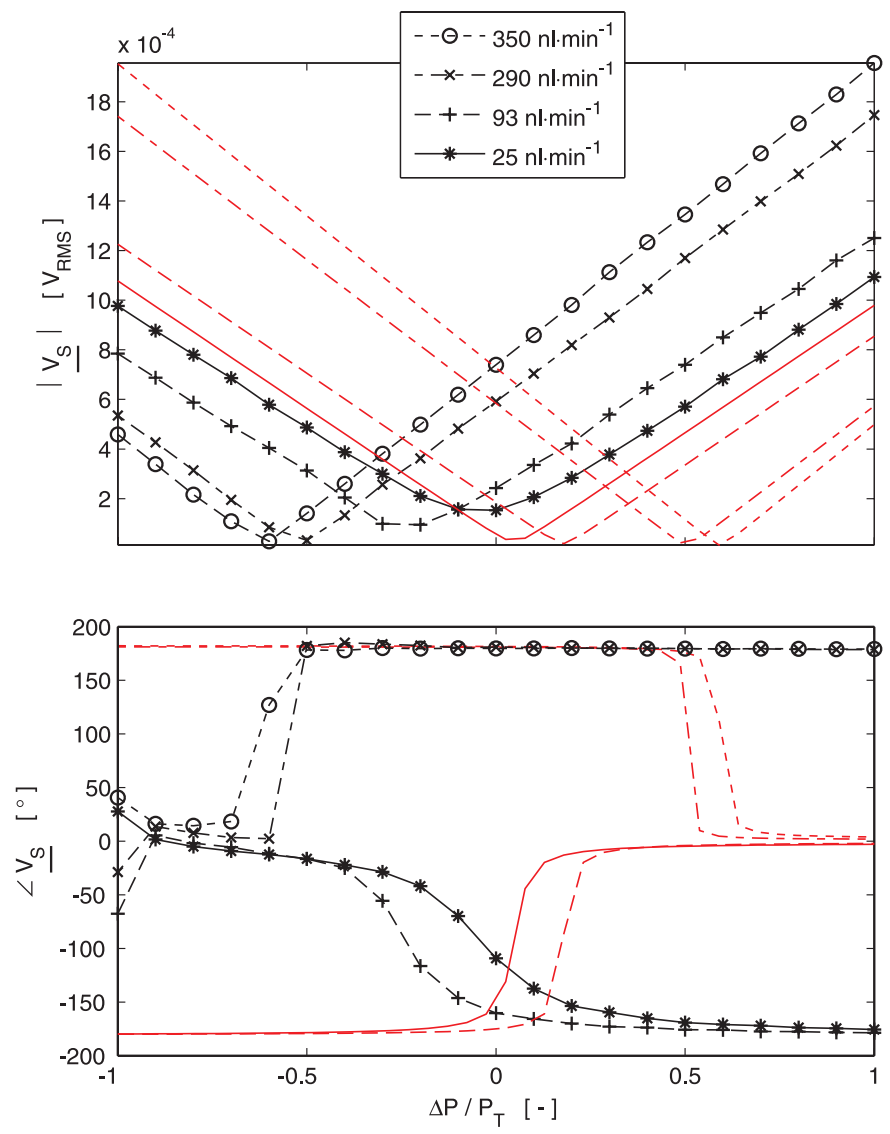

Fig.8.9 Measured and calculated open-loop sensor response on power difference $\triangle P$ at various water flow rates. 
ous water flow rates. These open-loop measurements show that it is difficult to use the phase of $\underline{V}_{s}$ as a control signal. In Fig 8.8 the phase decreases, with increasing frequency. The FVM model predicts that this is also dependent on the type of fluid in the microchannel. This is because thermal conduction and heat capacity determine the phase of the temperature at the heater resistors, which does not need to be exactly the same as the phase in the power dissipated in the heaters. Figure 8.9 shows that the amplitude of $\underline{V}_{s}$ at various flow rates has a clearly defined minimum, which can be obtained by using a control system.

Figure 8.10 shows the sensor response of the flow sensor obtained by determining the $\Delta P$ required for minimum amplitude of $\underline{V}_{s}$ for various flow rates. The sensor output $\Delta P / P_{T}$ shows to be independent of operation frequency as well as total heating power. A nearly linear sensor output is obtained up to about $500 \mathrm{nl} \cdot \mathrm{min}^{-1}$. The FVM model accurately indicates that at higher flow rates temperature gradients near the silicon substrate force the heater temperature to decrease (Fig. 8.5).

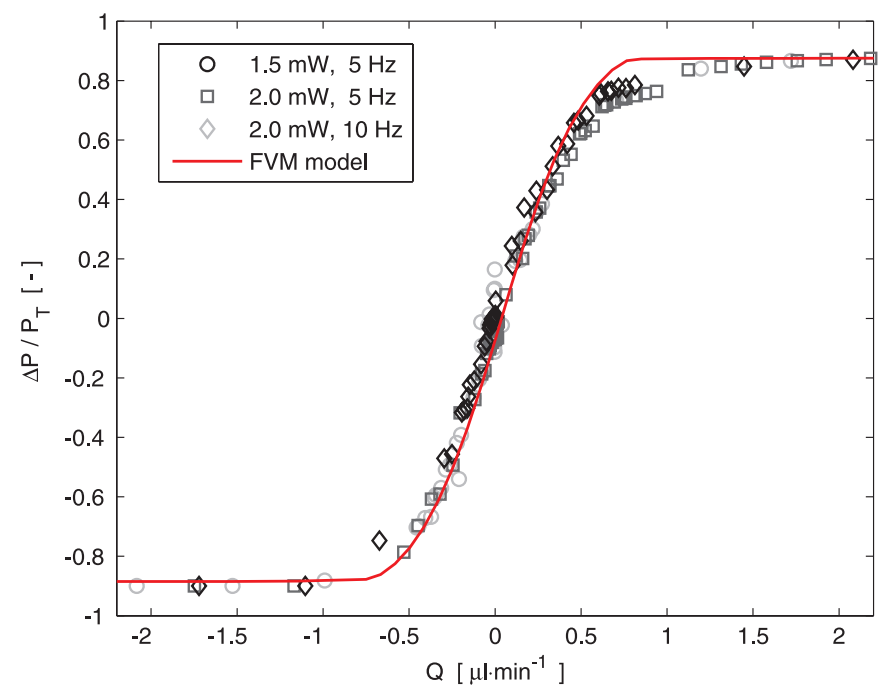

Fig. 8.10 Sensor output $\triangle P / P_{T}$ response on water flow rate $Q$ at different frequencies and total heating power, compared to FVM model results.

A control-system was implemented based on gradient descent to obtain $\Delta P$ at minimum amplitude of $\underline{V}_{s}$ (Fig. 8.11). The gradient is determined by two measurement points $\left(\Delta P_{n} \pm \delta P\right)$ close to $\Delta P_{n}$. Using small step-size for convergence, $\Delta P_{n+1}$ is placed in opposite direction to the gradient. Convergence can be slow when $\Delta P_{n}$ is far from minimum amplitude. A direct linear fit to find the minimum, when large changes in flow rate occur, can be made if during sensor operation an estimate 

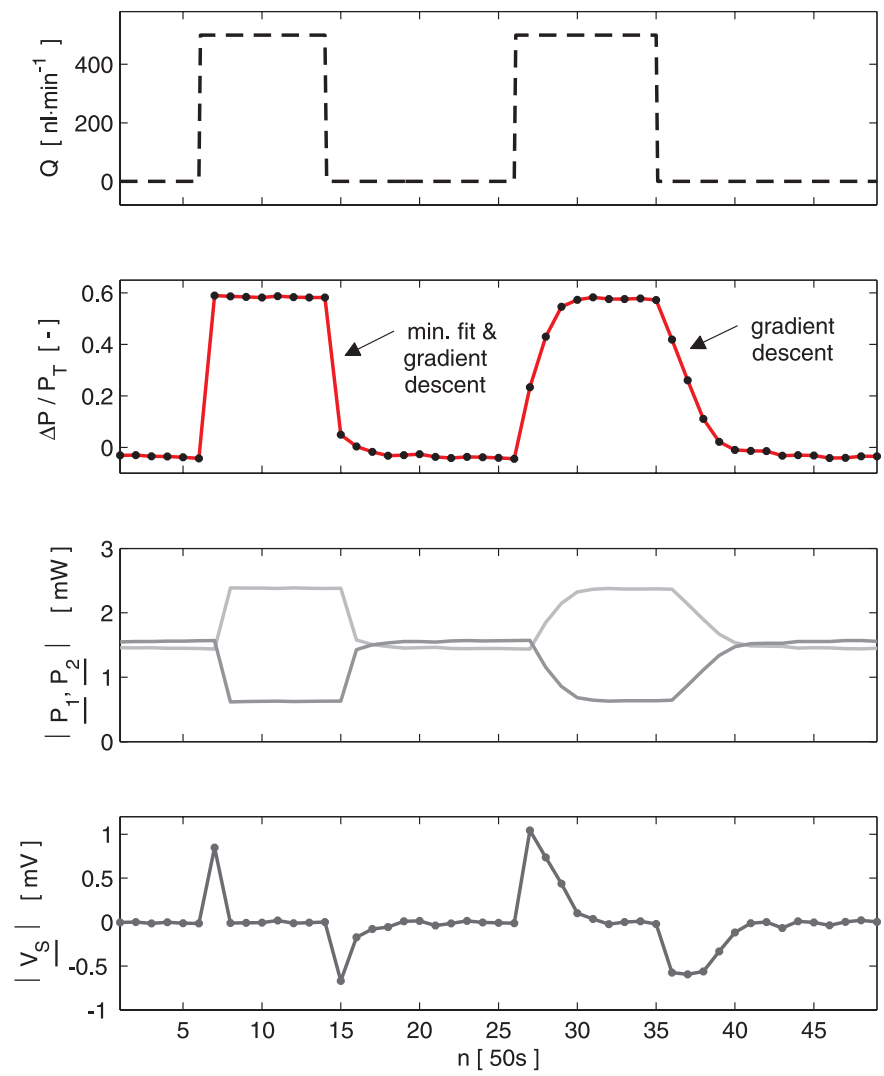

Fig. 8.11 Sensor control-system response on changing flow rate $Q$ at $3 \mathrm{~mW}$ total heating power $P_{T}$. During the second change in flow only the gradient descent method is used, resulting in a significant slower response.

of the absolute value of the minimum amplitude of $\underline{V}_{s}$ can be made. Accurate convergence has to be obtained by gradient descent, if the direct fit was not entirely accurate. The question remains how to optimally decide at what change in flow rate the linear fit should be applied. Fig. 8.11 shows that direct linear fit combined with gradient descent has a much faster response than when only using gradient descent. A sample time $n$ of only 50 s could be obtained. This is mostly limited by the DSP lockin amplifier (SRS 830). Much faster sampling can be obtained using dedicated electronics. Ultimately, the sample time is limited by the frequency of the heat waves measured with the lock-in amplifier, where an optimum has to be found between required sample time and the decrease in amplitude measured by the sensor resistor.

A drift measurement for $498 \mathrm{hrs}$ was conducted, without water in the microchannel in an ambient environment. Figure 8.12 shows the power spectral density of the heater resistors and the drift in the sensor output $\Delta P$. The heater resistors clearly show 


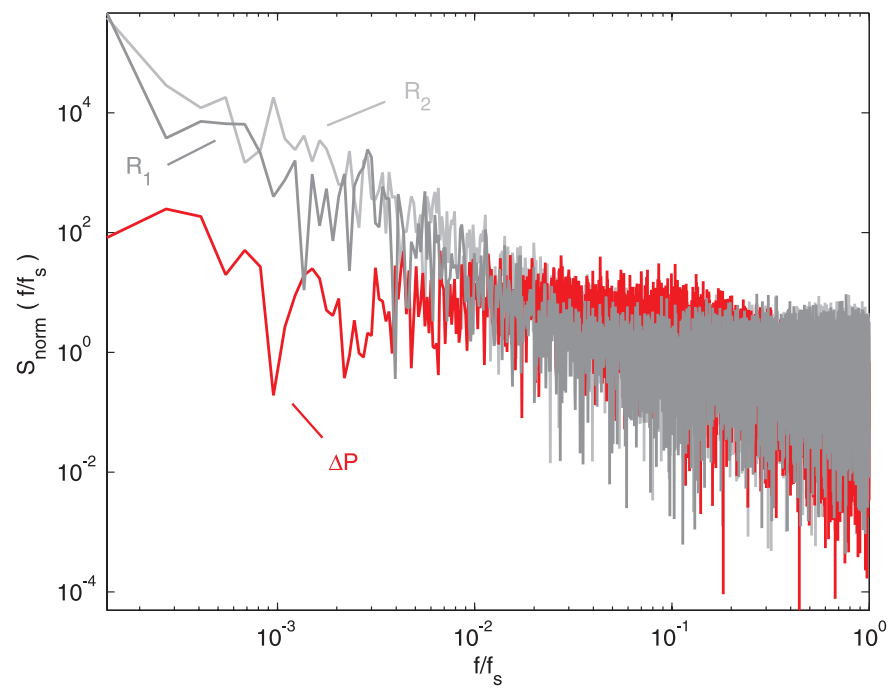

Fig. 8.12 Drift measurement for 498 brs with $8.2 \cdot 10^{-3} \mathrm{~Hz}_{\mathrm{z}}$ sampling frequency $f_{\text {s, }}$ showing normalised power spectral density $S_{\text {norm }}$ at $2 \mathrm{~mW}$ total power $P_{T}$.

$1 / f^{\alpha}$ noise spectra, while the power spectral density for $\Delta P$ is nearly flat. The power spectral density for $\Delta P$ is even slightly flatter than in Fig. 5.11. Measurement points of $\Delta P$ indicate that thermal gradients over the chip are not measured. For instance, no day-night rhythm was observed in the time signal of $\Delta P$, while it can be observed in a change in resistance values of the heater resistors.

\subsection{Conclusions}

A microchannel thermal flow sensor with integrated $\mathrm{Al}$ resistors has successfully been fabricated. The flow sensor is capable of measuring water flow rates up to about $500 \mathrm{nl} \cdot \mathrm{min}^{-1}$, using the AC-driven temperature-balancing concept [8.5], providing a sensor output independent of operation frequency, resistance values and total heating power. Thermal operation of the flow sensor is well understood by the dynamic FVM model of the flow sensor, giving exact fits to measurement results. A control-system based on gradient descent shows stable operation independent of operation frequency and thermal properties. Optimisation of the control system is shown to be feasible. The obtained drift performance is similar, if not better, to the sensor design with thermopiles discussed in Chap. 5. A disadvantage is the slower response time, because relatively low frequencies have to be used in combination with long averaging times. Effects of thermal gradient are however eliminated with a relatively simple sensor structure. 


\section{References}

[8.1] M. Dijkstra, T.S.J. Lammerink, O. Pjetri, M.J. de Boer, J.W. Berenschot, R.J. Wiegerink, M. Elwenspoek, “AC-driven temperature-balancing flow sensor with zero-offset power feedback”, Proc. MME, (2008).

[8.2] M. Dijkstra, M.J. de Boer, J.W. Berenschot, T.S.J. Lammerink, R.J. Wiegerink, M. Elwenspoek, "Miniaturized thermal flow sensor with planar integrated sensor structures on semicircular surface channels", Sensor. Actuat. A-Phys., 143 (2008) 1-6.

[8.3] M. Dijkstra, T.S.J. Lammerink, M.J. de Boer, J.W. Berenschot, R.J. Wiegerink, M. Elwenspoek, "Low-drift flow sensor with zero-offset thermopile-based power Feedback", Proc. DTIP, (2008).

[8.4] T.S.J. Lammerink, N.R. Tas, J.W. van Honschoten, G.J.M. Krijnen, J.J. van Baar, M. Elwenspoek, "AC-driven temperature-balance Flow Sensor", Proc. Transducers, (2001).

[8.5] T.S.J. Lammerink, N.R. Tas, G.J.M. Krijnen, M. Elwenpoek, "A new class of thermal flow sensors using $\Delta \mathrm{T}=0$ as a control signal", Proc. IEEE MEMS, Miyazaki, Japan (2000) 525-530.

[8.6] P. Bruschi, A. Diligente, D. Navarrini, M. Piotto, "A double heater integrated gas flow sensor with thermal feedback", Sensor. Actuat. A-Phys., (2005) 123-124.

[8.7] M. Dijkstra, M.J. de Boer, J.W. Berenschot, T.S.J. Lammerink, R.J. Wiegerink, M. Elwenspoek, "A versatile surface channel concept for microfluidic applications", J. Micromech. Microeng., 17 (2007) 1971-1977. 


\section{Nano-Nozzle Electrospray Emitters Fabricated by a Micro- to Nano-Fluidic Via Technology}

This chapter describes nano-nozzle electrospray emitters fabricated by a micro- to nanofluidic via technology. The main advantage of the technology is the ability to integrate freely-suspended nanochannels anywhere on a micro-fluidic chip. Nano-nozzle electrospray emitters were created on freely suspended microchannels. Leak-tight delivery of fluid from a fluidic reservoir was established through long microchannels. It was observed that other mechanisms aside from bulk capillary action contribute to filling of nanochannels coupled to microchannels. Stable electrospray IV-curves could be obtained from fabricated nano-electrospray emitters. 9.1

\subsection{Introduction}

Nano-fluidic tip based applications have emerged in the form of nano-fluidic fountain pens [9.1-9.3] and nano-electrospray emitters [9.4-9.6]. These applications require the delivery of fluid to functional nano-fluidic tips, possibly arranged in large arrays. Fluidic connection all the way up to the tip has mostly [9.7] been achieved by capillary slots or by extending nanochannels from fluid reservoirs [9.1-9.6]. In this chapter a fluidic via technology is described that allows for the coupling of microchannels to nanochannels. Microchannel fluidic networks are created beneath the wafer surface, facilitating the leak-tight chemical-inert delivery of fluid from macro-fluidic connections to nanochannels anywhere on the wafer surface. The mechanical strength of the microchannels can furthermore be utilised in making long freely suspended probes, with a nanochannel positioned right at the tip. The microchannels deliver fluid at low hydraulic resistance and large fluid volume ratios compared to connected nanochannels.

In this chapter nano-nozzle electrospray emitters are described, fabricated by the via technology. S. Koster and E. Verpoorte [9.8] presented a detailed review on microfluidics coupled to electrospray mass spectrometry (ESI-MS) [9.9-9.11]. A clear trend in on-chip integrated electrospray emitters was observed. In electrospray ionisation (ESI), reducing emitter aperture size is actively being pursued [9.5, 9.6], because of smaller droplets being generated. The enhanced ionisation efficiency obtained is of use to proteomics [9.12] and metabolomics. The via technology de-

\footnotetext{
9.1 This chapter is to be submitted to Lab on a Chip.
} 
scribed in this chapter, provides a method to combine small aperture-size emitters, with microfluidics in a monolithically-integrated electrospray chip.

\subsection{Experimental}

\subsubsection{Micro- to Nano-Fluidic Vias}

Microchannel fluidic networks are created beneath the wafer surface for the on-chip delivery of bulk fluid, applying the surface channel concept [9.13]. Microfluidic devices (e.g. a thermal flow sensor [9.14]) can be integrated with nanochannels using the via technology described in this chapter. The microchannels are formed by isotropic dry-etched of the silicon wafer through $2 \mu \mathrm{m}$ wide etch holes in a $500 \mathrm{~nm}$ silicon-rich silicon nitride (SiRN) layer. The etch holes are closed by a second $1.3 \mu \mathrm{m}$ SiRN low-pressure chemical vapour deposition (LPCVD), while the inside surface of the etched profile in the silicon wafer is simultaneously conformally coated [9.13].

Fluidic vias are created by selectively designed etch holes $3 \mu \mathrm{m}$ wide, preventing them from closing during SiRN deposition. Plugs are inserted in the open vias by $1 \mu \mathrm{m}$ poly-Si LPCVD deposition and patterning (Fig. 9.1a), where the deposition rate inside the microchannels was found to decrease with distance from the via.

The sealed microchannels leave a nearly planar wafer surface on which sacrificial nanochannels $(4 \mu \mathrm{m} \times 200 \mathrm{~nm})$, with $200 \mathrm{~nm}$ SiRN capping layer, are connected to the poly-Si plugs (Fig. 9.1b). Nano-lithographical methods could be applied to integrate nanochannels with even smaller dimensions.

Electrospray chips were released using $25 \mathrm{wt} \% \mathrm{KOH}$ at $75^{\circ} \mathrm{C}$ [9.15], where the sacrificial poly-Si is etched after inlets and outlets have been created (Fig. 9.1c). Only

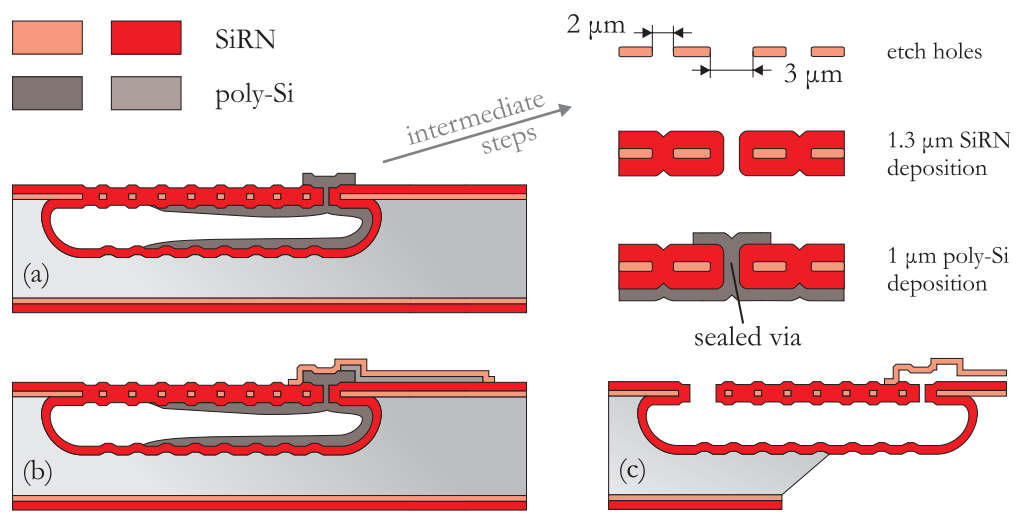

Fig. 9.1 Process schematic for the fabrication of via connections between surface microchannels [9.13] and nanochannels, with a) fabrication of via (see intermediate steps), b) fabrication of nanochannels and c) top-and bottom side SiRN etching of SiRN layers, where simultaneously openings are etched in the micro- and nanochannel, followed by $\mathrm{KOH}$ release of the electrospray chip. 


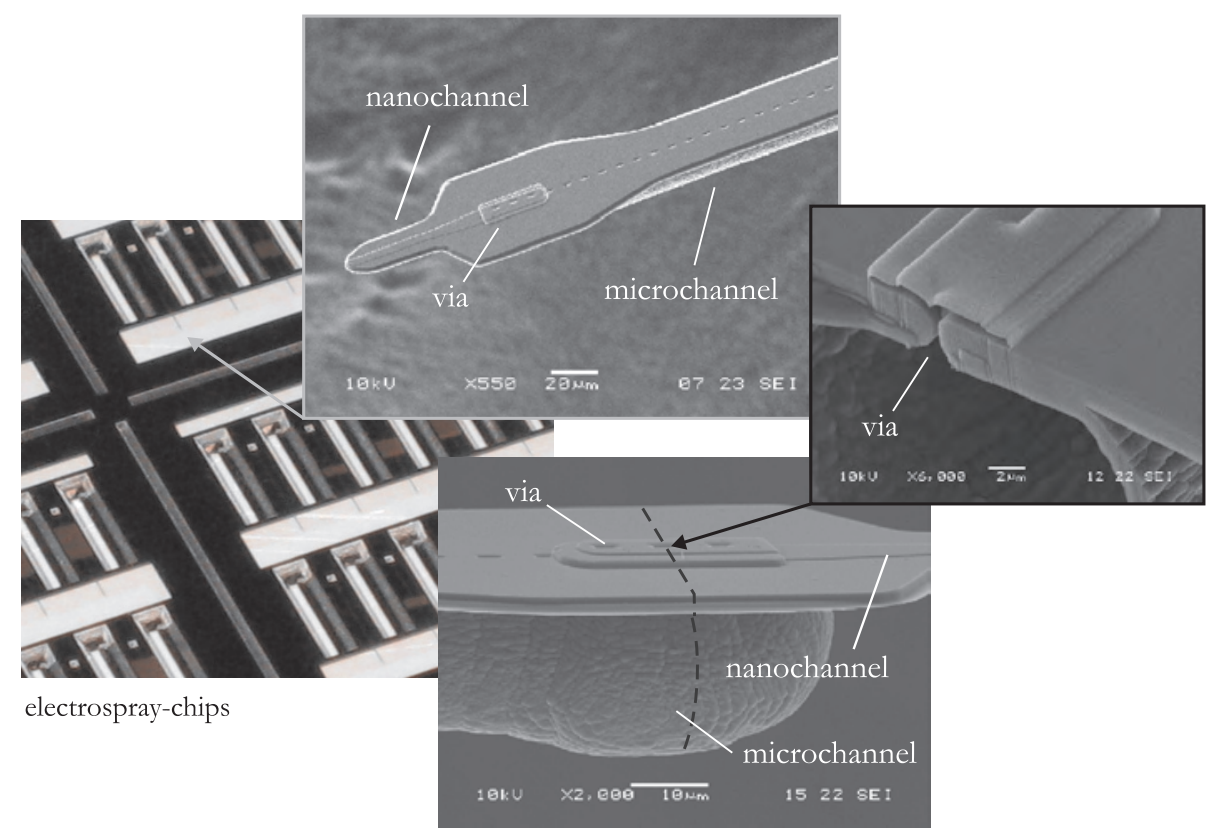

Fig. 9.2 Nanochannel electrospray emitters, with via connection between micro- and nanochannels. The rightmost inset shows a FIB cut through a fluidic via.

in cases where etching occurs from a nanochannel into a sealed microchannel without an outlet, it was observed that via connections break. This is caused by a sudden increase in $\mathrm{H}_{2}$ gas produced due to a large surface array of poly-Si being exposed.

Figure 9.2 shows a fabricated electrospray probe with a $60 \mu \mathrm{m}$ long nanochannel, where the inset shows a focused ion-beam (FIB) cut through the fluidic via connection between micro- and nanochannel.

\subsubsection{Filling Experiments}

Capillary filling of the electrospray tip (Fig. 9.2) was attempted by applying deionised (DI) water at the inlet. Capillary filling of the microchannel was found to be restricted by the large volume of air in the microchannel, which needs to be pushed out through the nanochannel. This was confirmed by preparing several nanochannel lengths from the initial $60 \mu \mathrm{m}$ long nanochannel (Fig. 9.2), where the filling speed was found to be constant in time during restricted capillary filling and inversely proportional to the nanochannel length. Subsequent refilling of previously filled electrospray chips resulted in instant wetting of the nanochannel with the formation of bubbles along the microchannel, likely due to an already saturated atmosphere present in the microchannel. 


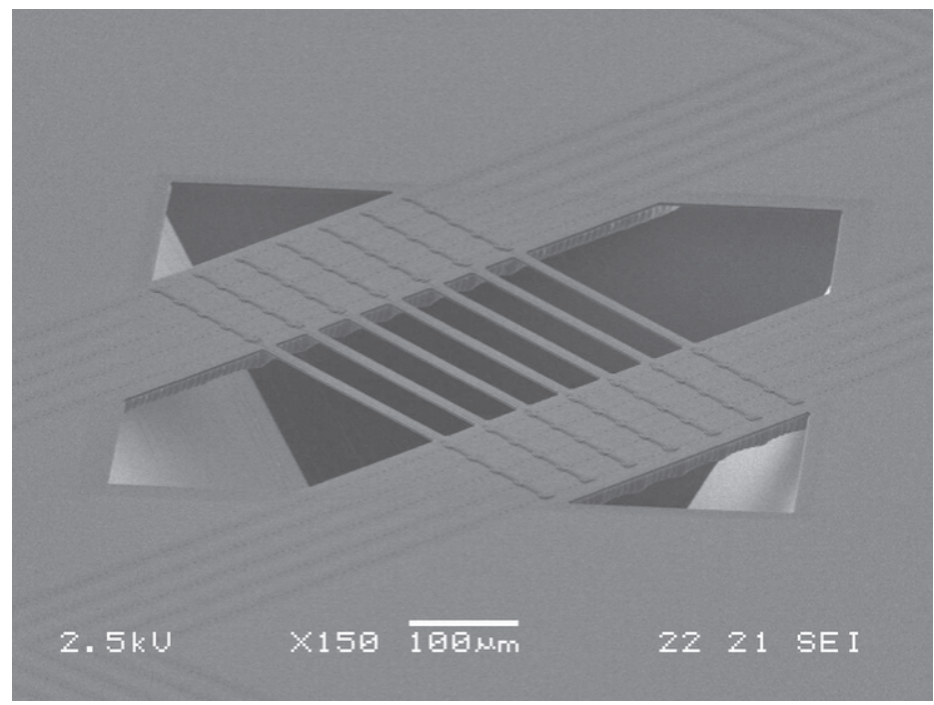

Fig. 9.3 Micrograph of freely suspended nanochannels connected to microchannels for on-chip transport of fluid.

A fluidic chip was fabricated to study filling mechanisms, containing freely suspended nanochannels, connected to separate microchannels on either side for the transport of fluid (Fig. 9.3). The on-chip freely-suspended nanochannels could be used for fluid-resonance applications. D. Westberg et al. [9.16] have created a fluid density sensor using sacrificial nanochannels and T.P. Burg et al. [9.17] have used nanochannel cantilevers for the weighing of biomolecules and single cells.

The filling of the chip was studied by applying DI water at the inlets of the microchannels connected to the lower-right part of the nanochannels in Figs. 9.3-9.4, while the microchannels on the upper-left part were not filled.

Figure 9.4 shows subsequent snapshots taken, during filling of the chip, where after 1.6 $\mathrm{s}$ the fluid reaches the via connections through a crossflow, where the air in the microchannels is pushed out through the microchannel outlets. The fluid then enters through the fluidic vias into the nanochannels. Liquid appears on the opposite ends of the nanochannels first, just before bulk fluid with a visible corner flow [9.18] enters the nanochannels from the wetted microchannels at $3.8 \mathrm{~s}$. The unexpected filling observed, with liquid appearing on opposite ends first, is likely caused by invisible transport mechanisms, such as vapour diffusion, film flow and corner flow [9.19]. After $6.1 \mathrm{~s}$ air bubbles remain, which slowly dissolve until the nanochannels are completely filled at $17.3 \mathrm{~s}$. The pinning of the fluid contact line [9.20] at the fluidic via connections on the opposite ends of the nanochannels prevents further capillary filling of the connected microchannels. 

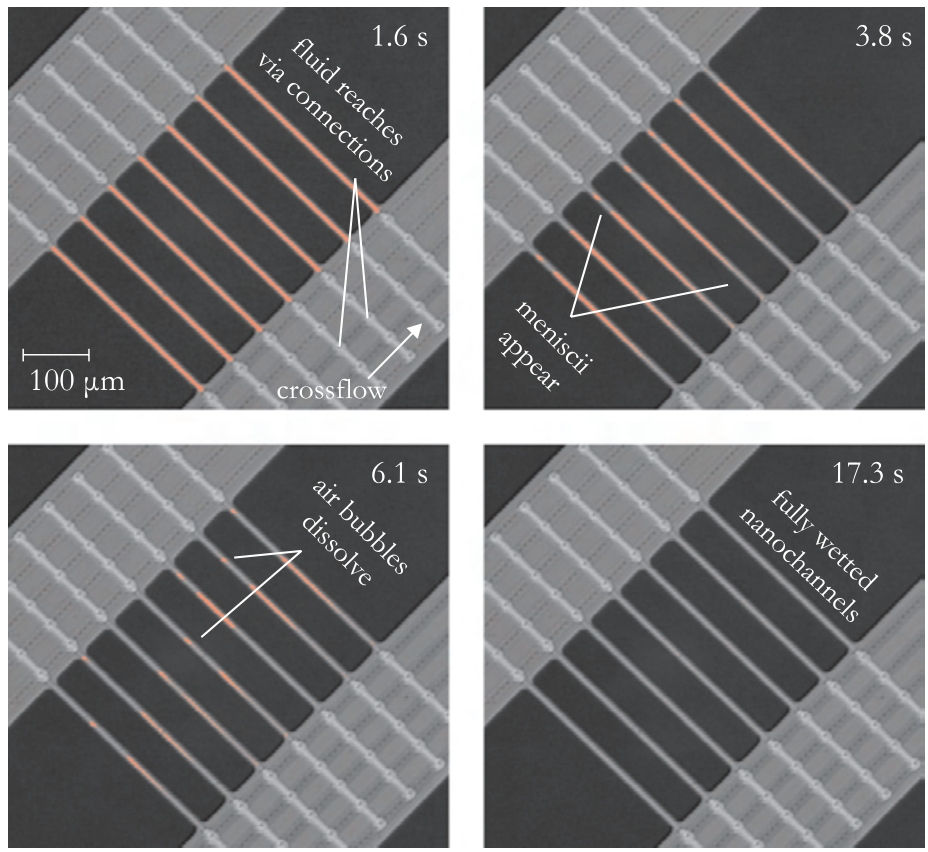

Fig. 9.4 Snapshots taken during the filling with DI water of freely suspended nanochannels. Each frame has been enhanced with the same image filter.

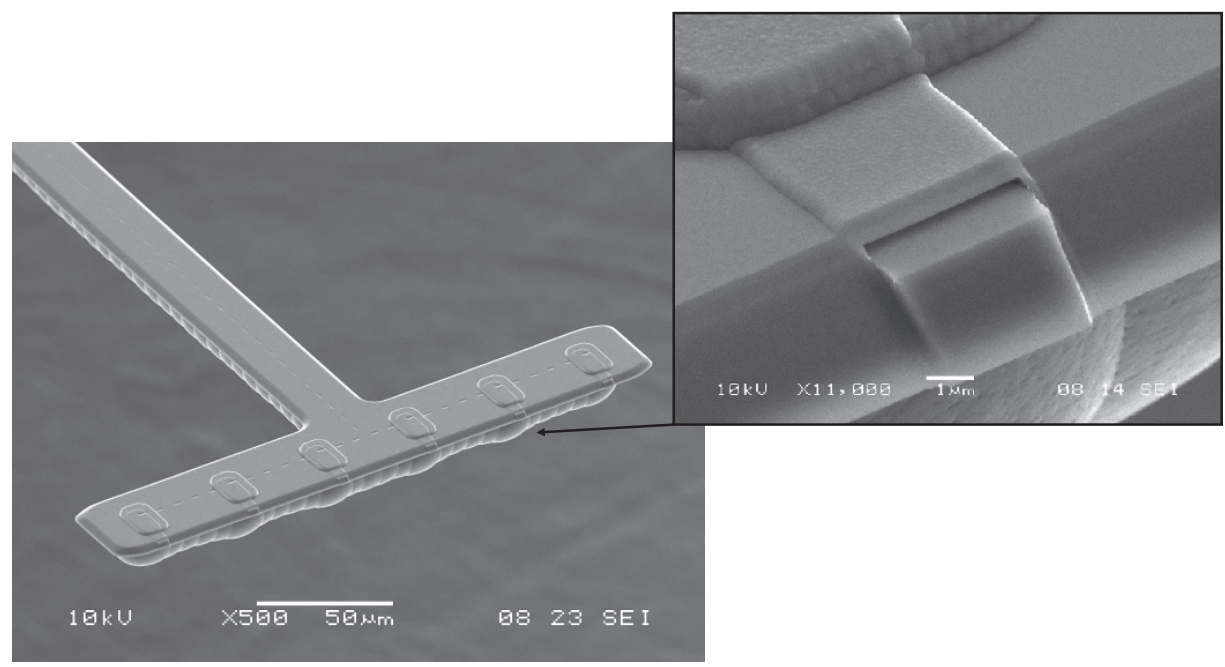

Fig. 9.5 Micrographs of nano-nozzle electrospray emitters on a microchannel probe. The inset shows a nano-nozzle with $4 \mu \mathrm{m} \times 200 \mathrm{~nm}$ aperture size. The poly-Si acts as a delayed mask during SiRN etching, resulting in a protrusion near the nozzle opening. 


\subsubsection{Nano-Nozzle Electrospray Emitters}

A redesign of the nanochannel electrospray emitter was made based on experimental filling results, taking vapour diffusion, corner flow, film flow [9.18-9.19] and gas-flow restriction into consideration. Electrospray chips were batch fabricated containing microchannel probes with integrated nano-nozzle emitters, where the nanochannel length was designed to be $3 \mu \mathrm{m}$ (Fig. 9.5), facilitating the fast filling of the microchannel. With the redesign, electrospraying can be performed from multiple emitters for higher spray throughput, while the air inside the microchannel can easily escape during capillary filling of the fluidic chip.

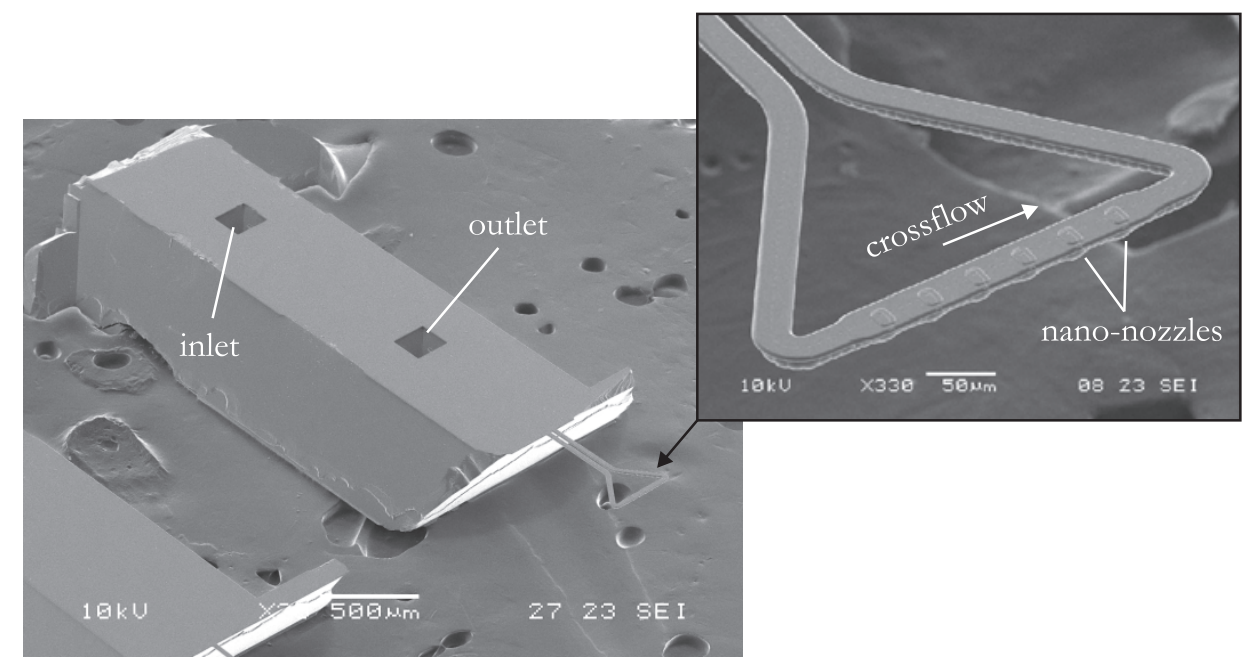

Fig. 9.6 Micrographs of nano-nozzle electrospray emitters, where a crossflow can pass the nano-nozzles from an on-chip inlet to an outlet.

Additional designs were made, where the nano-nozzles are integrated on a continuous microchannel, such that a crossflow can pass the nozzles (Fig. 9.6). This eliminates problems with air bubbles remaining in the microchannel and even electrospraying from a continuous flow can be achieved.

\subsubsection{Electrospray-Ionisation Setup}

Both the nanochannel electrospray chip after FIB cutting (Fig. 9.2) and the nanonozzle emitter chip (Fig. 9.5) were used for electrospraying. A Keithley 237 highvoltage source-measurement unit (SMU) was used to apply an electric field (Fig. 9.7). Triax-cable connections with active guarding were used for high spray-current resolution. The electrospray chip was filled with spraying solution and a probe needle 

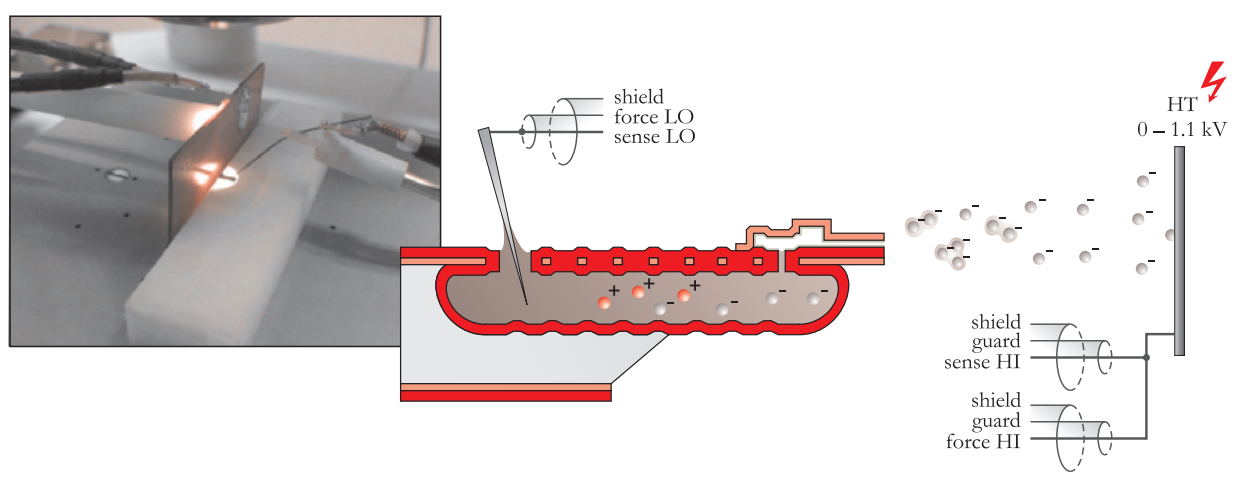

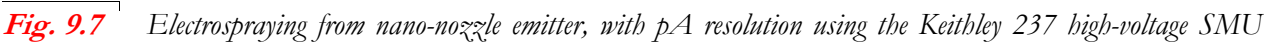
and triax-cable connections.

was inserted in the inlet. The counter electrode was isolated from the rest of the setup using a Teflon rod, with at least several $\mathrm{T} \Omega$ resistance. The counter electrode distance to the emitter was determined using an adjustable micrometer. With the setup, pA resolution was obtained up to $1.1 \mathrm{kV}$ counter electrode voltage applied. Without spraying, IV-curves show hysteresis due to capacitive effects in cabling and the solution inside the microchannel. Therefore, an $8 \mathrm{~s}$ delay was used between applying counter electrode voltage and acquisition of the spray current.

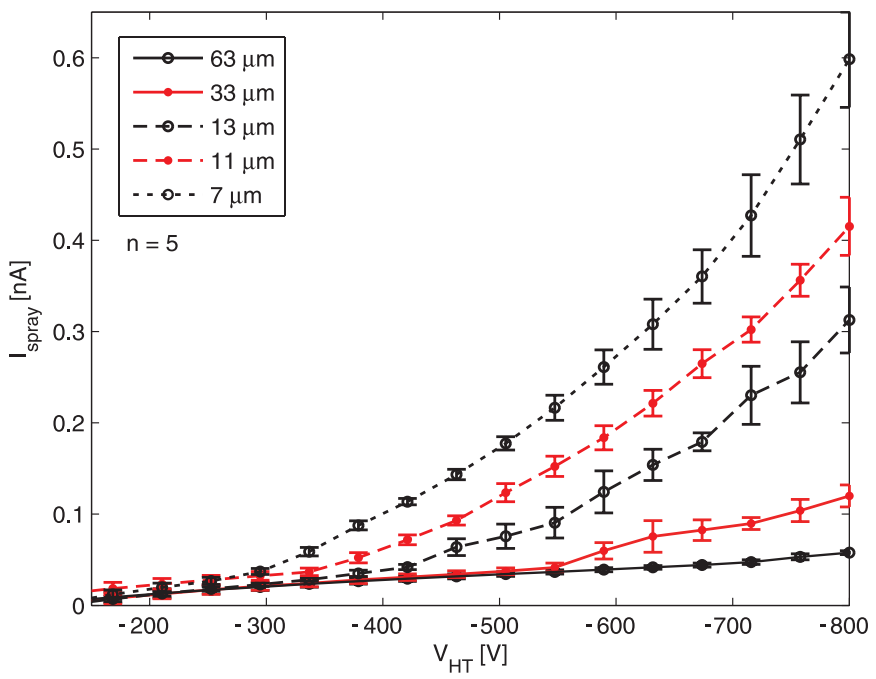

Fig. 9.8 Electrospray IV-curves of fully wetted FIB-cut 15 um nanochannel emitter at various distances to the cathode, using $\mathrm{H}_{2} \mathrm{O}: \mathrm{MeOH}\left(50: 50 v^{\%} \%\right)$ solution $\left(4.6 \mu \mathrm{S} \cdot \mathrm{cm}^{-1}\right)$. 


\subsection{Results and Discussion}

Electrospray IV-curves were obtained on a fully-wetted nanochannel emitter (Fig. 9.2) reduced in length to $15 \mu \mathrm{m}$, using $\mathrm{H}_{2} \mathrm{O}: \mathrm{MeOH}$ (50:50v\%) solution, without back pressure applied (Fig. 9.8). Reproducible IV-curves in the $\mathrm{nA}$ range were obtained at various distances of the emitter to the cathode. The spray current is generally very stable (small standard deviation) near the onset of spraying. The spray current shows a near exponential relation at higher spray voltages.

Several nano-nozzle emitter chips (Fig. 9.5) were filled with $\mathrm{H}_{2} \mathrm{O}: \mathrm{MeOH}(50: 50 \mathrm{v} \%$ ) solution for spraying. However, during capillary filling the solution did not fully wet the nano-nozzle emitters. The microchannels always filled completely, but the fluid contact line did not cross the fluidic vias. The fluidic chip with freely suspended nanochannels (Fig. 9.3), also fabricated on the same wafer, did however fill with the same $\mathrm{H}_{2} \mathrm{O}: \mathrm{MeOH}(50: 50 \mathrm{v} \%)$ solution. It is likely, considering filling experiment results discussed earlier, that the fluid contact line in both cases is pinned on the fluidic vias, which can be overcome by the development of a saturated atmosphere in the nanochannel. This can explain the unsuccessful filling of the $3 \mu \mathrm{m}$ short nano-nozzle emitters, due to a high evaporation rate, such that a saturated atmosphere cannot develop. Remarkably spraying does occur when using a high-conductive $\mathrm{H}_{2} \mathrm{O}$ : $\mathrm{MeOH}(50: 50 \mathrm{v} \%)+\mathrm{CH}_{3}-\mathrm{COOH}(1 \mathrm{v} \%)$ solution, where electrospray IV-curves could be obtained from non fully-wetted nano-nozzle emitters (Fig. 9.5). The exact spraying

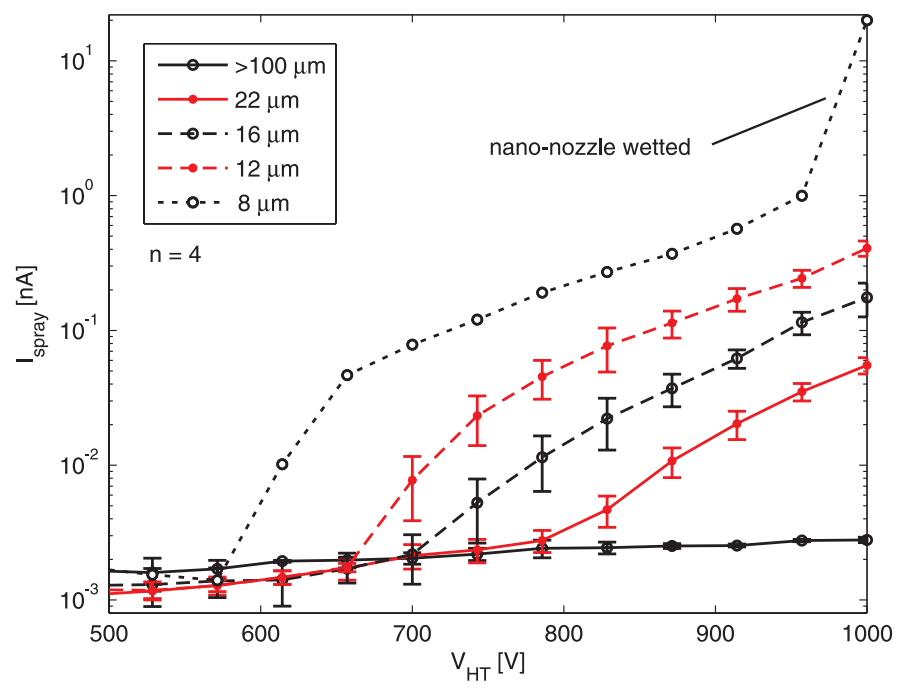

Fig. 9.9 Electrospray IV-curves of non fully-wetted nano-nozzle emitters at various distances to the anode, using $\mathrm{H}_{2} \mathrm{O}: \mathrm{MeOH}(50: 50 v \%)+\mathrm{CH}_{3} \mathrm{COOH}(1 v \%)$ solution $\left(120 \mu \mathrm{S} \cdot \mathrm{cm}^{-1}\right)$. 


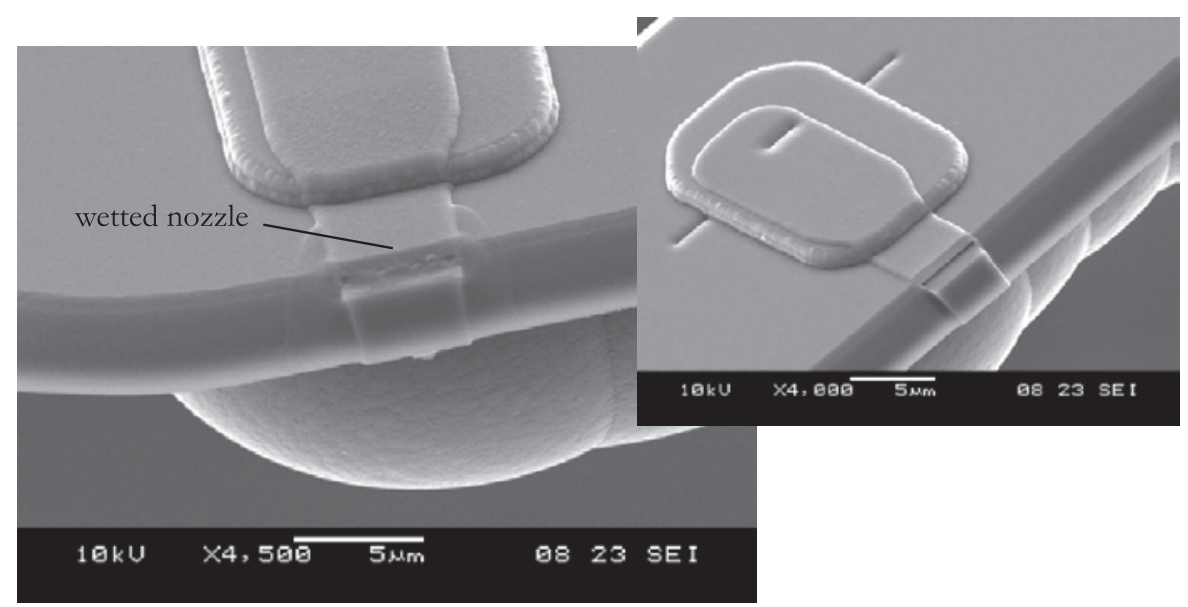

Fig. 9.10 Micrographs of the nano-nozzles, wetted after electrospraying at 8 um anode distance applying $1 \mathrm{kV}$ compared to non fully-wetted nozile.

mechanism is currently not understood. It is possible that nano-sized droplets are directly sprayed from the nano-nozzles emitters [9.21].

IV-curves, at various distances to the anode, were obtained (Fig. 9.9). During the last IV-curve measured at $8 \mu \mathrm{m}$ distance it was observed that the nano-nozzle closest to the anode, due to a small angular misalignment, became fully wetted. The strong electric field apparently pulled the fluid contact line through the fluidic via. The spray current instantly became $20 \mathrm{nA}$, which was set as current limit. The electrospray chip was taken for SEM inspection directly after the electrospray measurement. Figure 9.10 shows fluid in- and outside of the fully wetted nano-nozzle, after the sample had been SEM coated. The other nozzles were no different from nozzles inspected just after fabrication, confirming that the IV-curves in Fig. 9.8 were obtained on non fullywetted emitters.

\subsection{Conclusions}

A novel micro- to nano-fluidic via technology has been developed. It was demonstrated that bulk fluid can be transported from macro-fluidic connections to on-chip nanochannels. The technology has shown potential in applications were onchip freely suspended nanochannels are required. The capillary filling of fluidic chips was studied and it was concluded that vapour diffusion, corner flow, film flow, gasflow restriction and pinning of fluid contact lines have to be considered when designing coupled micro- to nano-fluidic devices. Better understanding of these transport mechanisms during filling is required for the via technology to be applicable to other fluidic designs. Proper capillary filling of microchannels can however be readily 
achieved by applying crossflow filling of connected nanochannels, such that the air in the microchannel can escape through a microchannel outlet.

Stable electrospray IV-curves were obtained from nano-nozzle emitters, despite the fact that the nozzles did not fully wet. This provides for an interesting spraying mode, which requires further investigation. Especially, the size distribution of possibly emitted nano-droplets, compared to fully wetted emitters, is of interest.

The prospect of the nano-nozzle electrospray chip is to increase emitter density, while reducing aperture size. Nano-droplet emission at higher densities can then be achieved. The nano-droplets emitted could be used for thin-film deposition. Thin-film deposition with large aperture-size emitters [9.22, 9.23] is limited by the need for the evaporation of solvents. R. Saf et al. [9.24] have used drying gas to dry of solvents and used electrostatic lenses for focussing, creating structured organic thin-films under atmospheric pressure.

The combination of the nano-nozzle emitters with surface microchannels allows for the integration of a high-pressure liquid chromatography (HPLC) column, where HPLC packing material can be packed up to the nano-nozzle emitters. Alternatively, capillary electrophoresis (CE) can be performed with on-chip.

\section{References}

[9.1] S. Deladi, N.R. Tas, J.W. Berenschot， G.J.M. Krijnen, M.J. de Boer, J.H. de Boer, M. Peter, M.C. Elwenspoek, "Micomachined fountain pen for atomic force microscope-based nanopatterning”, Appl. Phys. Lett., 85 (2004) 5361-5363.

[9.2] N. Moldovan, K-H. Kim, H.D. Espinosa, "A multi-ink linear array of nanofountain probes”, J. Micromech. Microeng., 16 (2007) 1935-1942.

[9.3] D. Saya, T. Leïchlé, J.B. Pourciel, C. Bergaud, L. Nicu, "Collective fabrication of an in-plane silicon nanotip for parallel femtoliter droplet deposition", J. Micromech. Microeng., 17 (2007) N1-N5.

[9.4] B. Legrand, A.E. Ashcroft, L. Buchaillot, S. Arscott, "SOI-based nanoelectrospray emitter tips for mass spectrometry: A coupled MEMS and microfluidic design", $J$. Micromech. Microeng., 17 (2007) 509-514.

[9.5] S. Arscott, D. Troadec, "Electrospraying from nanofluidic capillary slot", Appl. Phys. Lett., 87 (2005) 134101.

[9.6] E. Mery, F. Ricoul, N. Sarrut, O. Constantin, G. Delapierre, J. Garin, F. Vinet, “A silicon microfluidic chip integrating an ordered micropillar array separation column and nano-electrospray emitter for LC/MS analysis of peptides", Sensor. Actuat. BChem., 134 (2008) 438-446.

[9.7] T.S. Hug, N.F. de Rooij U. Staufer, "Fabrication and electroosmotic flow measurements in micro- and nanofluidic channels", Microfluid. Nanofluid., 2 (2006) 117-124.

[9.8] S. Koster, E. Verpoorte, "A decade of microfluidic analysis coupled with Electrospray mass spectrometry: An overview", Lab Chip, 7 (2007) 1394-1412. 
[9.9] J. Xie, Y. Miao, J. Shih, Y-C. Tai, T.D. Lee, "Microfluidic platform for liquid chromatography - tandem mass spectrometry analyses of complex peptide mixtures", Anal. Chem., 77 (2005) 6947-6953.

[9.10] P. Griss, "Micromachined interfaces for medical and biochemical applications", Ph. D. thesis (2002), Royal Institute of Technology, Stockholm, Sweden.

[9.11] M. Brivio, R.E. Oosterbroek, W. Verboom, A. van den Berg, D. N. Reinhoudt, "Simple chip-based interfaces for on-line monitoring of supramolecular interactions by nano-ESI MS", Lab Chip, 5 (2002) 1111-1112.

[9.12] G.A. Valaskovic, N.L. Kelleher, D.P. Little, D.J. Aaserud, F.W. McLafferty, "Attomole-sensitivity electrospray source for large-molecule mass spectrometry", Anal. Chem., 67 (1995) 3802-3805.

[9.13] M. Dijkstra, M.J. de Boer, J.W. Berenschot, T.S.J. Lammerink, R.J. Wiegerink, M. Elwenspoek, "A versatile surface channel concept for microfluidic applications", J. Micromech. Microeng., 17 (2007) 1971-1977.

[9.14] M. Dijkstra, M.J. de Boer, J.W. Berenschot, T.S.J. Lammerink, R.J. Wiegerink, M. Elwenspoek, "Miniaturized thermal flow sensor with planar integrated sensor structures on semicircular surface channels", Sensor. Actuat. A-Phys., 143 (2008) 1-6.

[9.15] J.W. Berenschot, N.R. Tas, T.S.J. Lammerink, M. Elwenspoek, A. van den Berg, "Advanced sacrificial poly-Si technology for fluidic systems", J. Micromech. Microeng., 12 (2002) 621-624.

[9.16] D. Westberg, O. Paul, G.I. Andersson, H. Baltes, “A CMOS-compatible device for fluid density measurements fabricated by sacrificial aluminium etching", Sensor. Actuat., 73 (1999) 243-251.

[9.17] T.P. Burg, M. Godin, S.M. Knudsen, W. Shen, G. Carlson, J.S. Foster, K. Babcock, S.R. Manalis, "Weighing of biomolecules, single cells and single nanoparticles in fluid”, Nature, 446 (2007) 1066-1069.

[9.18] M. Dong, I. Chatzis, "The imbition and flow of a wetting liquid along the corners of a square capillary tube”, J. Colloid Interf. Sci., 172 (1995) 278-288.

[9.19] J.C.T. Eijkel, B. Dan, H.W. Reemeijer, D.C. Hermes, J.G. Bomer, A. van den Berg, "Strongly accelerated and humidity-independent drying of nanochannels induced by sharp corners", Phys. Rev. Lett., 95 (2005) 1-4.

[9.20] T. Ondarçuhu, A. Piednoir, "Pinning of a contact line on nanometric steps during the dewetting of a terraced substrate", Nano Lett., 5 (2005) 1744-1750.

[9.21] R. Juraschek, T. Dülcks, M. Karas, "Nanoelectrospray-more than just a minimizedflow electrospray ionization source”, J. Am. Soc. Mass. Spectr., 10 (1999) 300-308.

[9.22] F. Schulz, S. Franzka, G. Schmid, "Nanostructured surface by deposition of metal nanoparticles by means of spray techniques", Adv. Funct. Mater., 12 (2002) 532-536.

[9.23] I.B. Rietveld, K. Kobayashi, H. Yamada, K Matsushige, "Morphology control of poly(vinylidene fluoride) thin film made with electrospray", J. Colloid Interf. Sci., 298 (2006) 639-651.

[9.24] R. Saf, M. Goriup, T. Steindl, T.E. Hamedinger, D. Sandholzer, G. Hayn, "Thin organic films by atmospheric-pressure ion deposition", Nat. Mater., 3 (2004) 323-329. 



\section{Conclusions and Future Research \\ In this final chapter conclusions are drawn on a number of important research results described in previous chapters. These results relate to: the fabrication technology used to fabricate the micro flow sensors presented in this thesis, improvements in accuracy by reduction of material drift influences and reduction of influences by external temperature gradients. The chapter ends with an outlook on possible future research in low-drift micro flow sensors.}

\subsection{Surface Channel Technology}

The existing buried channel technology [10.1] has been modified to make it suitable for application in flow sensors for the measurement of extremely small flow rates in the $\mathrm{nl} \cdot \mathrm{min}^{-1}$ range. The reason for developing the microchannel fabrication process was that previously used sacrificial layer based technology proved not to be suitable for these applications due to the large pressure drops associated with surface micromachined channels (see e.g. [10.2-10.4]). The developed surface channel technology (Chap. 3) allows for the fabrication of microchannels with semicircular cross sections, with low hydraulic resistance.

After fabrication of the microchannels a completely planar wafer surface remains, so that transducer structures can easily be integrated in close proximity to the fluid. The planar wafer surface allows for standard lithographic processing. Furthermore, high-temperature fabrication steps are possible, because the microchannels are made of low-stress silicon-rich silicon-nitride ( $\mathrm{SiRN}$ ). For example the $\mathrm{Al} / \mathrm{poly}_{-} \mathrm{Si}^{++}$thermopiles used in micro flow sensors presented in this thesis were made by solid-source diffusion (SSD) of boron at $1050{ }^{\circ} \mathrm{C}$, but also other high-temperature steps like wafer bonding have been demonstrated. Wafer level packaging of surface microchannel devices using wafer bonding is therefore possible and should be investigated further.

After integration of transducer structures the microchannels can easily be released by selectively etching the silicon substrate surrounding the microchannels. Wet etching in $\mathrm{KOH}$ can be used if this does not affect transducer material. Alternatively dry-etching can be used, where transducers are protected by photoresist during the release. In this thesis thermally-isolated freely suspended microchannels have been used in the fabrication of micro thermal flow sensors, but the microchannel technology can also be used in other applications that require freely suspended structures (e.g. micro heat exchangers, micro reactors or fluidic resonators $[10.5,10.6])$.

In Chap. 9 the surface channel technology is extended to include nanochannels that are fabricated on the wafer surface and connected to the semicircular microchannels 
using fluidic vias. The via technology was demonstrated with the realisation of nanonozzle electrospray emitters. The technology can be used in applications where micro fluidic components need to be integrated with nanofluidic channels (e.g. an HPLC column or a CE capillary with electrospray emitters). The combination of nanochannels with surface microchannels enables the fabrication of nanofluidic structures positioned at the tip of freely suspended microchannel probes.

\subsection{Material-Drift Compensation}

Drift in material properties of thin-film materials used in micro flow sensors is inevitable. Resistors are heated by Joule heating and the heating and current density cause an increase in $1 / f^{\alpha}$ noise, by the diffusion of material due to electromigration $[10.7,10.8]$. In this thesis it was shown that power control on heater resistors is very efficient in reducing influences of material drift in heater resistors (Chap. 5, 8). Power control requires the measurement of both the voltage across and the current through the resistor by four-point measurement, so that the resistance value can be calculated and the current adjusted accordingly. To avoid influence of thermocouple offset voltages on interface wires to the chip a heating current with alternating polarity has to be used. In this way resistance drift is largely cancelled. Drift by non-uniform changes in resistance material remains, because this may result in redistribution of the dissipated power on the heater resistor. This secondary effect has been observed at high-temperature (Fig. 5.12). It shows to be the main cause of residual sensor output fluctuation.

Although power control can effectively be used to eliminate $1 / f^{\alpha}$ noise in heating elements, a low-drift sensing element is required in order to obtain a low-drift flow sensor. Therefore, a thermopile was used as sensor element, which has inherently zero-offset if the thermopile is at a uniform temperature. Sensitivity drift of the thermopile remains, which can be eliminated by using a temperature-balancing control system as demonstrated in Chap. 5. The control system adjusts the heating powers such that the thermopile voltage is cancelled. The resulting heating powers are a measure for the flow, independent of the thermopile sensitivity.

Accurate read-out of the thermopile voltage requires that interface wires to the chip are close to each other in order to retain an almost equal temperature, avoiding offset voltages. Further improvement in reducing influence by interface offset voltages can be obtained by integrating more junctions in the thermopile (Chap. 6) and by using a low-drift or chopper amplifier close to the chip.

With interface offset voltages eliminated, temperature gradients along the thermopile leads in combination with material inhomogeneities (giving a non-uniform Seebeck coefficient along the leads) [10.9, 10.10] may still result in a thermopile offset voltage even when thermopile junctions on opposite sides are at the same 
temperature. As a result, temperature-balancing control can give zero thermopile voltage, but this does not necessarily correspond to zero temperature difference between the thermopile junctions. Therefore, a thermopile temperature-balancing flow sensor may still give drift in offset voltage in case of inhomogeneities being introduced in the thermopile leads. The long-term drift properties of the thermopiles in combination with temperature-balancing control has to be investigated further by extremely long-term drift measurements, much longer than required for long-term material drift to be apparent in heater resistors.

Another method to obtain a drift-free measure for temperature difference is to use a sensor resistor in combination with heat-waves. This possibility was explored in Chap. 8 and it was shown that similar and possibly better long-term accuracy can be achieved at the expense of a slower response time of the sensor.

\subsection{Temperature-Gradient Compensation}

In Chap. 5 it was shown that drift in sensor materials can be effectively eliminated. However, the sensor output appeared to be dependent on temperature gradients induced by changes in ambient temperature (e.g. day-night rhythm was visible in the sensor output). Additional measurements in an oven also showed that the oven temperature can modulate thermal gradients over the chip, due to differences in heat leakage to ambient through interface wires going outside the oven. With material drift largely compensated, the drift performance of the micro flow sensor was now limited by these external temperature-gradient effects.

To solve this problem a U-shaped flow sensor was designed (Chap. 6). This resulted in a significant improvement, although conduction through the surrounding air still resulted in an output from the thermopile in the presence of a thermal gradient across the chip. To fully eliminate the influence of externally induced gradients a completely symmetrical flow sensor was designed (Chap. 7).

Also the sensor based on heat-waves (Chap. 8) is independent of thermal gradients over the chip, because only heat-waves at the lock-in frequency are detected.

\subsection{Outlook on Low-Drift Micro Flow Sensing}

Long-term flow sensor accuracy has been greatly enhanced by near complete elimination of drift factors (temperature-gradient and material-drift compensation). What remains to be optimised is sensor precision at a given acquisition rate for measuring liquid flows in the $\mathrm{nl} \cdot \mathrm{min}^{-1}$ range. Relatively low acquisition rates were obtained with measurement equipment used during measurements presented in this thesis. The acquisition rates used are much slower than the time needed to obtain 
thermal equilibrium at a given power setting, which is in the order of milliseconds, allowing for enormous improvements in acquisition rate.

At the time of writing, a temperature-balancing control system is under investigation, built using zero-drift opamps (AD8628) and a data-acquisition board (NI-DAQ). Initial measurements show that temperature-balancing, using a linear least square method, and power control on heater resistors, with currents having alternating polarity, can be performed on flow sensors presented in Chap. 5 with $\mathrm{nl} \cdot \mathrm{min}^{-1}$ resolution at $0.5 \mathrm{~Hz}$ acquisition rate. The largest limiting factor in increasing precision and acquisition rate are external disturbances superimposed on the thermopile voltage, due to the relatively high thermopile impedance and the high-impedance opamp used.

The most important topic for further research is the improvement of the signal to noise ratio. Improvements are possible by using adequate electrical shielding and optimisation of thermopile materials, obtaining high sensitivity at low impedance. The total heating power can also be increased and should be optimised for life-time and reliability of the micro flow sensor.

Further investigation is needed to find an optimal flow sensor design. In Chap. 7 it was shown that a symmetrical design with a meandering microchannel can be used to fully compensate for external temperature gradients. However, for optimal low-drift performance every heater resistor should have individual power control, which requires the total number of heater resistors to be minimised. The minimal symmetric design, consisting of two parallel microchannels with opposite flow direction and independent power control on four heater resistors is therefore close to an optimal low-drift flow sensor design. An important aspect that should be investigated is how to obtain highest sensitivity. The two parallel microchannels allow thermopile leads to be suspended between the microchannels (as with the U-shaped sensor in Chap. 6) increasing the amount of thermocouple junctions, however at the expense of increased thermal conduction between the microchannels.

Finally, further research is needed to improve calibration of the micro flow sensors. The flow sensors presented in this thesis were calibrated using an elevation head, where a water container was put at a specific height compared to a container on a balance. This provided for a stable flow through the microchannel, however the exact flow rate, measured by measuring the weight on the balance, was still difficult to obtain at high precision. Typically in the order of $100 \mathrm{nl} \cdot \mathrm{min}^{-1}$ evaporation from the container on the balance was measured. Evaporation was largely compensated for by measuring the evaporation rate between each measurement point and long averaging time was used for flow-rate calibration. Improvements can be made by using a smaller container on the balance and by using a thin layer of oil on top of the fluid to reduce evaporation. The obtained maximum resolution of the flow sensors has so far been limited by the calibration setup. A calibration setup for accurately determining flow 
rates down to $1 \mathrm{nl} \cdot \mathrm{min}^{-1}$ and below will be a challenge, but is needed to accurately determine the resolution of the fabricated low-drift micro flow sensors.

\section{References}

[10.1] M.J. de Boer, R.W. Tjerkstra, J.W. Berenschot, H.V. Jansen, G.J. Burger, J.G.E. Gardeniers, M. Elwenspoek, A. van den Berg, "Micromachining of buried microchannels in silicon”, J. Microelectromech. S., 9 (2000) 94-103.

[10.2] M. Dijkstra, T.S.J. Lammerink, R.J. Wiegerink, M. Elwenspoek, "Nano-flow thermal sensors applying dynamic $\omega-2 \omega$ sensing method", Proc. MME, (2006) 29-32.

[10.3] S. Wu, Q. Lin, Y. Yuen, Y-C. Tai, "MEMS flow sensors for nano-fluidic applications", Sensor. Actuat. A-Phys., 89 (2001) 152-158.

[10.4] Y. Mizuno, M. Liger, Y-C. Tai, "Nanofluidic flowmeter using carbon sensing element", Proc. IEEE MEMS, (2004) 322-325.

[10.5] J. Haneveld, T.S.J. Lammerink, M. Dijkstra, H. Droogendijk, M.J. de Boer, R.J. Wiegerink, "Highly sensitive micro Coriolis mass flow sensor", Proc. IEEE MEMS (2008).

[10.6] J. Haneveld, T.S.J. Lammerink, M.J. de Boer, R.J. Wiegerink, "Micro Coriolis mass flow sensor with integrated capacitive readout", Proc. IEEE MEMS (2009).

[10.7] W. Yang, Z. Çelik-Butler, "A model for electromigration and low-frequency noise in thin metal films”, Solid State Electron., 34 (1991) 911-916.

[10.8] A. Bora, A.K. Raychaudhuri, "Evolution of $1 / \mathrm{f}^{\alpha}$ noise during elctromigration stressing of metal film: Spectral signature of electromigration process", J. Appl. Phys., 99 (2006) 113701.

[10.9] F. Jahan, M. Ballico, "Overcoming inhomogeneity and hysteresis limitations of type R thermocouples in an international comparison", Int. J. Thermophys., 28 (2007) 1832-1842.

[10.10] M. Holmsten, J. Ivarsson, R. Falk, M. Lidbeck, L-E. Josefson, “Inhomogeneity measurements of long thermocouples using a short movable heating zone", Int. J. Thermophys., 29 (2008) 915-925. 



\section{A.1 Surface Microchannel Fabrication}

\begin{tabular}{|c|c|c|c|}
\hline Nr. & Process & Process Parameters & $\begin{array}{c}\text { Design related parameters } \\
\text { and remarks }\end{array}$ \\
\hline 1. & $\begin{array}{l}\text { Substrate selection - } \\
\text { Silicon }<100>\text { OSP } \\
(\# \text { subs001) }\end{array}$ & $\begin{array}{l}\text { CR112B / Wafer Storage Cupboard } \\
\text { Orientation: }<100> \\
\text { Diameter: } 100 \mathrm{~mm} \\
\text { Thickness: } 525 \mu \mathrm{m}+/-25 \mu \mathrm{m} \\
\text { Polished: Single side } \\
\text { Resistivity: } 5-10 \Omega \cdot \mathrm{cm} \\
\text { Type: p }\end{array}$ & $\begin{array}{l}\text { nr. wafers:? } \\
\text { nr. dummies:? }\end{array}$ \\
\hline 2. & $\begin{array}{l}\text { Cleaning Standard } \\
(\# \text { clean003) }\end{array}$ & $\begin{array}{l}\text { CR112B / Wet-Bench } 131 \\
\mathrm{HNO}_{3}(100 \%) \text { Selectipur: MERCK } \\
\mathrm{HNO}_{3}(69 \%) \text { VLSI: MERCK } \\
\text { - Beaker 1: fumic } \mathrm{HNO}_{3}(100 \%), 5 \mathrm{~min} \\
\text { - Beaker 2: fumic } \mathrm{HNO}_{3}(100 \%), 5 \mathrm{~min} \\
\text { - Quick Dump Rinse }<0.1 \mu \mathrm{S} \\
\text { - Beaker 3: boiling }\left(95^{\circ} \mathrm{C}\right) \mathrm{HNO}_{3}(69 \%), 10 \mathrm{~min} \\
\text { - Quick Dump Rinse }<0.1 \mu \mathrm{S} \\
\text { - Spin drying }\end{array}$ & \\
\hline 3. & $\begin{array}{l}\text { Etching HF (1\%) } \\
\text { Native Oxide } \\
(\# \text { etch027) }\end{array}$ & $\begin{array}{l}\text { CR112B / Wet-Bench 3-3 } \\
\text { HF }(1 \%) \text { VLSI: MERCK } 112629.500 \\
\text { - Etch time: }>1 \text { min } \\
\text { - Quick Dump Rinse }<0.1 \mu \mathrm{S} \\
\text { - Spin drying }\end{array}$ & \\
\hline 4. & $\begin{array}{l}\text { LPCVD SiRN - low- } \\
\text { depostion rate } \\
\text { (\#depo002) }\end{array}$ & $\begin{array}{l}\text { CR125C / Tempress LPCVD new system } 2007 \\
\text { program: SiRN01/N2 } \\
\text { Tube: G3 } \\
\text { - "Use } 5-8 \text { boat fillers in front and back of the } \\
\text { boat to achieve specifications." } \\
\text { - } \mathrm{SiH}_{2} \mathrm{Cl}_{2} \text { flow: } 77.5 \mathrm{sccm} \\
\text { - } \mathrm{NH}_{3} \text { flow: } 20 \mathrm{sccm} \\
\text { - temperature: } 820 / 850 / 870{ }^{\circ} \mathrm{C} \\
\text { - pressure: } 150 \mathrm{mTorr} \\
\text { - deposition rate: } \pm 4 \mathrm{~nm} \cdot \mathrm{min}^{-1} \\
\text { - Nf: } \pm 2.18 \\
\text { - Stress (range): } 200-280 \mathrm{Mpa} \\
\text { - Boat position } 12: 200 \mathrm{MPa} \text { (centre of the boat) } \\
\text { - Boat position } 1: 280 \mathrm{MPa} \text { (front of the boat) } \\
\text { - Uniformity/wafer: }<2 \% \\
\text { - Uniformity over the boat: (20 wafers): }<8 \%\end{array}$ & $\begin{array}{l}\text { layer thickness: } 500 \mathrm{~nm} \\
\text { program: SIRN01 } \\
\text { nom. deposition rate: } \\
\quad 3.62 \mathrm{~nm} \cdot \mathrm{min}^{-1} \\
\text { nom. deposition time: } \\
2 \mathrm{hr} 20 \mathrm{~min}\end{array}$ \\
\hline 5. & $\begin{array}{l}\text { Ellipsometer } \\
\text { Measurement } \\
\text { (\#char007) }\end{array}$ & CR118B / Plasmos Ellipsometer & \\
\hline 6. & $\begin{array}{l}\text { Sputtering of Cr } \\
\text { (Sputterke) } \\
\text { (\#depo017) }\end{array}$ & $\begin{array}{l}\text { Eq.Nr. } 37 \text { / Sputterke } \\
\text { Cr Target (gun \#: “see mis logbook") } \\
\text { - Electrode temp.: water cooled electrode } \\
\text { - Ar flow: app. } 80 \mathrm{sccm} \text {, pressure depending! } \\
\text { - Base pressure: }<1.0 \cdot 10^{-6} \mathrm{mbar} \\
\text { - Sputter pressure: } 6.6 \cdot 10^{-3} \mathrm{mbar} \\
\text { - power: } 200 \mathrm{~W} \\
\text { - Depositionrate: } 15 \mathrm{~nm} \cdot \mathrm{min}^{-1}\end{array}$ & $\begin{array}{l}\text { layer thickness: } 50 \mathrm{~nm} \\
\text { nom. deposition time: } \\
\quad 4 \text { min } 10 \mathrm{~s} \\
\text { "ACr mask is used for proper } \\
\text { definition of the } 2 \text { m wide etch } \\
\text { boles." }\end{array}$ \\
\hline
\end{tabular}




\begin{tabular}{|c|c|c|c|}
\hline 7. & $\begin{array}{l}\text { Lithography - Vapor } \\
\text { prime HMDS } \\
\text { (gas phase) (\#lith002) }\end{array}$ & $\begin{array}{l}\text { CR112B / Furnace Vapor HMDS } \\
\text { HexaMethylDiSilazane (HMDS) } \\
\text { - Furnace: Lab-line Duo-vac-oven } \\
\text { - "See manual for operating the system." } \\
\text { - Dehydration bake }\left(150{ }^{\circ} \mathrm{C} \text {, low pressure): } 30 \text { min }\right. \\
\text { - Vapor prime HMDS at } 150^{\circ} \mathrm{C}: 5 \mathrm{~min} \\
\text { - "Before coating let cooldown the wafers to room } \\
\text { temperature." }\end{array}$ & \\
\hline 8. & $\begin{array}{l}\text { Lithography - } \\
\text { Coating Olin907-17 } \\
\text { (\#lith005) }\end{array}$ & $\begin{array}{l}\text { CR112B / Suss Micro Tech Spinner (Delta 20) } \\
\text { Hotplate } 95^{\circ} \mathrm{C} \text { Olin } 907-17 \\
\text { - Spin Program: } 4(4000 \mathrm{rpm}, 20 \mathrm{~s}) \\
\text { - Prebake }\left(95^{\circ} \mathrm{C}\right): 90 \mathrm{~s}\end{array}$ & \\
\hline 9. & $\begin{array}{l}\text { Lithography - } \\
\text { Alignment \& } \\
\text { Exposure Olin 907-17 } \\
\text { (EV620) } \\
\text { (\#lith021) }\end{array}$ & $\begin{array}{l}\text { CR117B / EV620 } \\
\text { Electronic Vision Group } 620 \text { Mask Aligner } \\
\text { - Hg-lamp: } 12 \mathrm{~mW} \cdot \mathrm{cm}^{-2} \\
\text { - Exposure Time: } 4 \mathrm{~s}\end{array}$ & $\begin{array}{l}\text { mask: CHNL recipe: first mask } \\
\text { exposure time: } 3.5 \mathrm{~s} \\
\text { mask: } 5 \text { " } 2.3 \mathrm{~mm} \\
\text { sub: } 4 \text { " } 0.53 \mathrm{~mm} \\
\text { contact: vacuum } \\
\text { flat rotation angle: } 0^{\circ} \\
\text { "Use EV with robot arm to achieve } \\
\text { good }<100>\text {-alignment.", "Check. } \\
\text { the vacuum ring." }\end{array}$ \\
\hline 10. & $\begin{array}{l}\text { Lithography - } \\
\text { Development Olin } \\
\text { Resist } \\
\text { (\#lith011) }\end{array}$ & $\begin{array}{l}\text { CR112B / Wet-Bench } \mathbf{1 1} \\
\text { Developer: OPD } 4262 \\
\text { Hotplate } 120^{\circ} \mathrm{C}(\mathrm{CR} 112 \mathrm{~B} \text { or CR117B) } \\
\text { - After Exposure Bake }\left(120^{\circ} \mathrm{C}\right): 60 \mathrm{~s} \\
\text { Development: } \\
\text { - Time: } 30 \mathrm{~s} \text { in Beaker } 1 \\
\text { - Time: } 15-30 \mathrm{~s} \text { in Beaker } 2 \\
\text { - Quick Dump Rinse }<0.1 \mu \mathrm{S} \\
\text { - Spin drying }\end{array}$ & $\begin{array}{l}\text { bkr1 develop time: } 30 \mathrm{~s} \\
\text { bkr2 develop time: } 30 \mathrm{~s}\end{array}$ \\
\hline 11. & $\begin{array}{l}\text { Optical microscopic } \\
\text { inspection - } \\
\text { Lithography } \\
\text { (\#char001) }\end{array}$ & CR112B / Nikon Microscope & $\begin{array}{l}\text { "The width of the etch holes must be } \\
\text { close to } 2 \text { um." }\end{array}$ \\
\hline 12. & $\begin{array}{l}\text { Ozone anneal of Olin } \\
907 \text { (to improve } \\
\text { wetting) } \\
\text { (\#lith038) }\end{array}$ & $\begin{array}{l}\text { CR116B-1 / UV PRS-100 } \\
\text { "To improve wetting during etching of Chromium layers." } \\
\text { • time: } 300 \mathrm{~s}\end{array}$ & $\begin{array}{l}\text { "Improves wetting of the Olin907- } \\
17 \text { resist." }\end{array}$ \\
\hline 13. & $\begin{array}{l}\text { Etching of Cr Wet } \\
\text { (\#etch034) }\end{array}$ & $\begin{array}{l}\text { CR116B / Wet-Bench } 2 \\
\text { Chromium etch LSI Selectipur: } \\
\text { MERCK } 111547.2500 \\
\text { - Quick Dump Rinse }<0.1 \mu \mathrm{S} \\
\text { - Spin drying } \\
\text { Etchrates: } 100 \mathrm{~nm} \cdot \mathrm{min}^{-1}\end{array}$ & $\begin{array}{l}\text { etch time: } 30 \mathrm{~s} \\
\text { "Prewet wafers in DI water before } \\
\text { Cr etcbing to further improve wetting } \\
\text { of the Olin907-17 resist.", } \\
\text { "Minimise underetch after } 30 \text { s by } \\
\text { QDR." }\end{array}$ \\
\hline 14. & $\begin{array}{l}\text { Optical microscopic } \\
\text { inspection - } \\
\text { Lithography } \\
\text { (\#char001) }\end{array}$ & CR112B / Nikon Microscope & "No Cr underetch should be visible." \\
\hline 15. & $\begin{array}{l}\text { Plasma etching - } \\
\text { chamber cleaning } \\
\text { (Etske) } \\
\text { (\# etch003) }\end{array}$ & $\begin{array}{l}\text { CR102A / Elektrotech PF310/340 } \\
\text { Select chamber for desired etch process } \\
\text { Select electrode for desired etch process } \\
\text { - Electrode temp.: } 10^{\circ} \mathrm{C} \text { or } 25^{\circ} \mathrm{C} \\
\text { - } \mathrm{O}_{2} \text { flow: } 20 \mathrm{sccm} \\
\text { - pressure: } 50 \mathrm{~m} \text { Torr } \\
\text { - power: } 150 \mathrm{~W} \\
\text { - DC-Bias: } 780 \mathrm{~V} \\
\text { "Chamber is clean when plasma color is white." }\end{array}$ & "Use quartz electrode." \\
\hline 16. & $\begin{array}{l}\text { Plasma etching SiN } \\
\text { (Etske) } \\
\text { (\#tch004) }\end{array}$ & $\begin{array}{l}\text { CR102A / Elektrotech PF310/340 } \\
\text { Dirty chamber } \\
\text { Styros electrode } \\
\text { - Electrode temp:: } 10{ }^{\circ} \mathrm{C} \\
\text { - } \mathrm{CHF}_{3} \text { flow: } 25 \mathrm{sccm} \\
\text { - } \mathrm{O}_{2} \text { flow: } 5 \mathrm{sccm} \\
\text { - pressure: } 10 \mathrm{~m} \text { Torr }\end{array}$ & $\begin{array}{l}\text { layer thickness: } 500 \mathrm{~nm} \\
\text { min. etch time: } 8 \mathrm{~min} 30 \mathrm{~s} \\
\text { nom. etch time: } 10 \mathrm{~min} \\
\text { "Use quartz electrode." }\end{array}$ \\
\hline
\end{tabular}




\begin{tabular}{|c|c|c|c|c|c|}
\hline & & \multicolumn{3}{|c|}{$\begin{array}{l}\text { - power: } 75 \mathrm{~W} \\
\text { Etchrate } \mathrm{SiN}: 50 \mathrm{~nm} \cdot \mathrm{min}^{-1}\left(\text { for } \mathrm{V}_{\mathrm{DC}}=-460 \mathrm{~V}\right) \\
\left.\text { Etchrate } \mathrm{SiN}: 75 \mathrm{~nm} \cdot \mathrm{min}^{-1} \text { (for } \mathrm{V}_{\mathrm{DC}}=-580 \mathrm{~V}\right) \\
\text { Etchrate Olin resist : } 95 \mathrm{~nm} \cdot \mathrm{min}^{-1} \\
\text { "If DC-Bias < } 375 \mathrm{~V} \text { apply chamber clean (\#etcb003)." }\end{array}$} & \\
\hline \multirow[t]{11}{*}{17.} & \multirow[t]{11}{*}{$\begin{array}{l}\text { Argon Sputter etched } \\
\text { (\#Etch074) }\end{array}$} & \multicolumn{3}{|c|}{$\begin{array}{l}\text { CR 125c/Adixen SE } \\
\text { Application: removal of native oxide } \\
\text { and/or residue }\end{array}$} & \\
\hline & & Parameters & \multicolumn{2}{|c|}{ Value } & \\
\hline & & Gas & \multicolumn{2}{|l|}{$\operatorname{Ar}$} & \\
\hline & & Flow [sccm] & \multicolumn{2}{|l|}{20} & \\
\hline & & Time [s] & \multicolumn{2}{|l|}{ ? } & \\
\hline & & $\mathrm{APC}[\%]$ & \multicolumn{2}{|l|}{100} & \\
\hline & & ICP [W] & \multicolumn{2}{|l|}{500} & \\
\hline & & CCP [W] RF & \multicolumn{2}{|l|}{10} & \\
\hline & & $\mathrm{SH}[\mathrm{mm}]$ & \multicolumn{2}{|l|}{200} & \\
\hline & & Electrode temp. $\left[{ }^{\circ} \mathrm{C}\right]$ & \multicolumn{2}{|l|}{+20} & \\
\hline & & He [bar] & \multicolumn{2}{|l|}{10} & \\
\hline \multirow[t]{14}{*}{18.} & \multirow{14}{*}{$\begin{array}{l}\text { Plasma etching } \\
\text { Silicon for semi- } \\
\text { isotropic channels } \\
\text { (SCT) } \\
\text { (\#)tch063) }\end{array}$} & \multicolumn{3}{|c|}{$\begin{array}{l}\text { CR 125c/Adixen AMS } 100 \text { SE } \\
\text { Applications: semi-isotropic channels (SCT) } \\
\text { Pre-etch step: same values, incl. CCP of } 30 \mathrm{~W} \text {, } \\
\text { time } 30 \text { s. }\end{array}$} & \multirow[t]{14}{*}{$\begin{array}{l}\text { etch rate: } 1.2 \mu \mathrm{m} \cdot \mathrm{min}^{-1} \\
\text { radius: ? } \\
\text { etch time: ? }\end{array}$} \\
\hline & & \multicolumn{2}{|l|}{ Parameters } & Value & \\
\hline & & \multicolumn{2}{|l|}{$\mathrm{SF}_{6}[\mathrm{sccm}]$} & 400 & \\
\hline & & \multicolumn{2}{|c|}{ He-backside cooling [mbar] } & 10 & \\
\hline & & \multicolumn{2}{|l|}{ ICP [W] } & 2500 & \\
\hline & & \multicolumn{2}{|l|}{ CCP On/Off [ms] } & 0 & \\
\hline & & \multicolumn{2}{|l|}{$\mathrm{p}[\mathrm{Pa}]$} & 10 & \\
\hline & & \multicolumn{2}{|l|}{ APC position [\%] } & 100 & \\
\hline & & \multicolumn{2}{|c|}{ Electrode temperature $\left[{ }^{\circ} \mathrm{C}\right]$} & 20 & \\
\hline & & \multicolumn{2}{|l|}{ Substrate height $[\mathrm{mm}]$} & 110 & \\
\hline & & \multirow{2}{*}{\multicolumn{2}{|c|}{$\begin{array}{l}\text { Etch rate } \mathrm{SiO} 2\left[\mu \mathrm{m} \cdot \mathrm{min}^{-1}\right] \\
\text { Etch rate Olin } 907\left[\mu \mathrm{m} \cdot \mathrm{min}^{-1}\right]\end{array}$}} & $?$ & \\
\hline & & & & 0.250 & \\
\hline & & \multicolumn{2}{|c|}{ Etch rate -Silicon $\left(\mu \mathrm{m} \cdot \mathrm{min}^{-1}\right)$} & $\begin{array}{l}1.2 \mu \mathrm{m} \cdot \mathrm{min}^{-1} \\
\text { (for } 2 \mu \mathrm{m} \text { slits- } \\
\text { SCT) }\end{array}$ & \\
\hline & & \multicolumn{2}{|c|}{ Etch rate SiRN $\left[\mathrm{nm} \cdot \mathrm{min}^{-1}\right]$} & 25 & \\
\hline 19. & $\begin{array}{l}\text { Stripping of polymers } \\
\text { in } \mathrm{NO}_{3} \text { multi- } \\
\text { purpose } \\
\text { (\#lith014) }\end{array}$ & \multicolumn{3}{|c|}{$\begin{array}{l}\text { CR116B / Wet-Bench } 2 \\
\mathrm{HNO}_{3}(100 \%) \text { Selectipur: MERCK } 100453 \\
\text { - Beaker 1: HNO } \mathrm{HN}_{3}(100 \%) 20 \mathrm{~min} \\
\text { - Quick Dump Rinse <0.1 } \mathrm{\mu S} \\
\text { - Spin drying }\end{array}$} & cleaning time: $20 \mathrm{~min}$ \\
\hline 20. & $\begin{array}{l}\text { Etching of Cr Wet } \\
(\# \text { etch034) }\end{array}$ & $\begin{array}{l}\text { CR116B / Wet-Bench } \\
\text { Chromium etch LSI Se } \\
111547.2500 \\
\text { - Quick Dump Rinse } \\
\text { - Spin drying } \\
\text { Etchrates: } 100 \mathrm{~nm} \cdot \mathrm{mir}\end{array}$ & $1 \mu \mathrm{S}$ & IERCK & $\begin{array}{l}\text { etch time: }>30 \text { min } \\
\text { "Periodically rinse with DI to remove } \\
\text { masking Cr-oxide." }\end{array}$ \\
\hline 21. & $\begin{array}{l}\text { Optical microscopic } \\
\text { inspection } \\
\text { (\#char002) }\end{array}$ & $\begin{array}{l}\text { CR112B / Nikon Mic } \\
\text { CR117B / Olympus I } \\
\text { CR102B / Olympus I }\end{array}$ & $\begin{array}{l}\text { scope } \\
\text { crosco } \\
\text { crosco }\end{array}$ & & $\begin{array}{l}\text { "Determine the LPCVD SiRN } \\
\text { layer thickness (balf of the etch bole } \\
\text { width }+0.2 \mu \mathrm{m} \text { ) required to seal the } \\
\text { surface microchannels." }\end{array}$ \\
\hline 22. & $\begin{array}{l}\text { Cleaning Standard } \\
(\# \text { clean003) }\end{array}$ & $\begin{array}{l}\text { CR112B / Wet-Bench } \\
\mathrm{HNO}_{3}(100 \%) \text { Selecti } \\
\mathrm{HNO}_{3}(69 \%) \text { VLSI: } \\
\text { - Beaker 1: fumic HNC } \\
\text { - Beaker 2: fumic HNC } \\
\text { - Quick Dump Rinse }\end{array}$ & $\begin{array}{l}31 \\
\text { : MER } \\
\text { RCK } \\
(100 \% \\
(100 \% \\
1 \mu S\end{array}$ & $\begin{array}{l}\mathrm{CK} \\
, 5 \mathrm{~min} \\
\end{array}$ & $\begin{array}{l}\text { nr. dummies: ? } \\
\text { "Dummies for ellipsometer } \\
\text { measurement." }\end{array}$ \\
\hline
\end{tabular}




\begin{tabular}{|c|c|c|c|}
\hline & & $\begin{array}{l}\text { - Beaker 3: boiling }\left(95^{\circ} \mathrm{C}\right) \mathrm{HNO}_{3}(69 \%), 10 \mathrm{~min} \\
\text { - Quick Dump Rinse }<0.1 \mu \mathrm{S} \\
\text { - Spin drying }\end{array}$ & \\
\hline 23. & $\begin{array}{l}\text { Etching HF (1\%) } \\
\text { Native Oxide } \\
(\# \text { etch027) }\end{array}$ & $\begin{array}{l}\text { CR112B / Wet-Bench 3-3 } \\
\text { HF }(1 \%) \text { VLSI: MERCK } 112629.500 \\
\text { - Etch time: }>1 \text { min } \\
\text { - Quick Dump Rinse }<0.1 \mu \mathrm{S} \\
\text { - Spin drying }\end{array}$ & \\
\hline 24. & $\begin{array}{l}\text { LPCVD SiRN - low- } \\
\text { depostion rate } \\
(\# \text { depo002) }\end{array}$ & $\begin{array}{l}\text { CR125C / Tempress LPCVD new system } 2007 \\
\text { program: SiRN01/N2 } \\
\text { Tube: G3 } \\
\text { - "Use } 5-8 \text { boat fillers in front and back of the } \\
\text { boat to achieve specifications." } \\
\text { - } \mathrm{SiH}_{2} \mathrm{Cl}_{2} \text { flow: } 77.5 \mathrm{sccm} \\
\text { - } \mathrm{NH}_{3} \text { flow: } 20 \mathrm{sccm} \\
\text { - temperature: } 820 / 850 / 870{ }^{\circ} \mathrm{C} \\
\text { - pressure: } 150 \mathrm{mTorr} \\
\text { - deposition rate: } \pm 4 \mathrm{~nm} \cdot \mathrm{min}^{-1} \\
\text { - Nf: } \pm 2.18 \\
\text { - Stress (range): } 200-280 \mathrm{Mpa} \\
\text { - Boat position } 12: 200 \mathrm{MPa} \text { (centre of the boat) } \\
\text { - Boat position } 1: 280 \mathrm{MPa} \text { (front of the boat) } \\
\text { - Uniformity/wafer: }<2 \% \\
\text { - Uniformity over the boat: (20 wafers): }<8 \%\end{array}$ & $\begin{array}{l}\text { nom. layer thickness: } 1.3 \mu \mathrm{m} \\
\text { program: SIRN01 } \\
\text { nom. deposition rate: } \\
\quad 3.62 \mathrm{~nm} \cdot \mathrm{min}^{-1} \\
\text { nom. deposition time: } 6 \mathrm{hrs} \\
\text { "Slowly (manually) move through the } \\
\text { front heatzone.", "Microchannels } \\
\text { should be dry to avoid rapid } \\
\text { evaporation of water." }\end{array}$ \\
\hline 25. & $\begin{array}{l}\text { Ellipsometer } \\
\text { Measurement } \\
\text { (\#char007) }\end{array}$ & CR118B / Plasmos Ellipsometer & $\begin{array}{l}\text { "Ellipsometer measurement on } \\
\text { dummies." }\end{array}$ \\
\hline 26. & $\begin{array}{l}\text { Optical microscopic } \\
\text { inspection } \\
(\# \text { char002) }\end{array}$ & $\begin{array}{l}\text { CR112B / Nikon Microscope } \\
\text { CR117B / Olympus Microscope } \\
\text { CR102B / Olympus Microscope }\end{array}$ & $\begin{array}{l}\text { "Rinse wafers in DI water and spin- } \\
\text { dry before inspection. Look for the } \\
\text { lack of water in the surface } \\
\text { microchannels, confirming proper } \\
\text { sealing of the microchannels." }\end{array}$ \\
\hline
\end{tabular}

\section{A.2 Al/ poly-Si ${ }^{++}$Thermopile Fabrication}

\begin{tabular}{|c|c|c|c|}
\hline Nr. & Process & Process Parameters & $\begin{array}{c}\text { Design related parameters } \\
\text { and remarks }\end{array}$ \\
\hline 1. & $\begin{array}{l}\text { Cleaning Standard } \\
(\# \text { clean003) }\end{array}$ & $\begin{array}{l}\text { CR112B / Wet-Bench } 131 \\
\mathrm{HNO}_{3}(100 \%) \text { Selectipur: MERCK } \\
\mathrm{HNO}_{3}(69 \%) \text { VLSI: MERCK } \\
\text { - Beaker 1: fumic } \mathrm{HNO}_{3}(100 \%), 5 \mathrm{~min} \\
\text { - Beaker 2: fumic } \mathrm{HNO}_{3}(100 \%), 5 \mathrm{~min} \\
\text { - Quick Dump Rinse }<0.1 \mu \mathrm{S} \\
\text { - Beaker 3: boiling }\left(95^{\circ} \mathrm{C}\right) \mathrm{HNO}_{3}(69 \%), 10 \mathrm{~min} \\
\text { - Quick Dump Rinse }<0.1 \mu \mathrm{S} \\
\text { - Spin drying }\end{array}$ & \\
\hline 2. & $\begin{array}{l}\text { Micro Balance } \\
\text { Measurement } \\
\text { (\#char006) }\end{array}$ & CR125C / Satotius Micro Balance & $\begin{array}{l}\text { "Measure weight of wafers before } \\
L P C V D \text { of poly-Si." }\end{array}$ \\
\hline 3. & $\begin{array}{l}\text { LPCVD Poly Si - } \\
\mathbf{5 9 0}^{\circ} \mathbf{C} \\
(\# \text { depo005) }\end{array}$ & $\begin{array}{l}\text { CR125C / Tempress LPCVD } \\
\text { Tube: F2 } \\
\text { Program: senspoly } \\
\text { - } \mathrm{SiH}_{4} \text { flow: } 50 \mathrm{sccm} \\
\text { - temperature: } 590^{\circ} \mathrm{C} \\
\text { - pressure: } 250 \mathrm{~m} \text { Torr } \\
\text { - deposition rate: } \mathrm{xx} \mathrm{nm} \cdot \mathrm{min}^{-1} \\
\text { - Stress: } 30 \mathrm{MPa}\end{array}$ & $\begin{array}{l}\text { layer thickness: } 200 \mu \mathrm{m} \\
\text { program: SENSPOLY } \\
\text { nom. depostion rate: } \\
\quad 3.33 \mathrm{~nm} \cdot \mathrm{min}^{-1} \\
\text { nom. depositon time: } 1 \mathrm{hr}\end{array}$ \\
\hline
\end{tabular}




\begin{tabular}{|c|c|c|c|}
\hline 4. & $\begin{array}{l}\text { Micro Balance } \\
\text { Measurement } \\
\text { (\#char006) }\end{array}$ & CR125C / Satotius Micro Balance & $\begin{array}{l}\text { "thickness }[n m]=26.5 \cdot \Delta m \\
{[m g] ", " 26.5 \text { is the conversion factor }} \\
\text { for } 4 \text { " wafers with LPCVD } \\
\text { poly-Si." }\end{array}$ \\
\hline 5. & $\begin{array}{l}\text { Solid Source } \\
\text { Diffusion (SSD) of } \\
\text { Boron at } 1050^{\circ} \mathrm{C} \\
\text { (\#dopi004) }\end{array}$ & $\begin{array}{l}\text { CR112B / Furnace B1 } \\
\text { Standby temperature: } 700{ }^{\circ} \mathrm{C} \\
\text { - Program: SSD-1050 } \\
\text { - Temp.: } 1050{ }^{\circ} \mathrm{C} \\
\text { - Gas N } 2: 30 \text { SLM } \\
\text { - Gas O }: 0.20 \mathrm{SLM}\end{array}$ & $\begin{array}{l}\text { doping time: } 45 \text { min } \\
\text { "Only use metal tweezers and fast } \\
\text { unload/load procedure.", "Etch } \\
\text { SSD dummies } 5 \text { min in BHF.", } \\
\text { "Old dummies require } 15 \text { min } \\
800^{\circ} \text { C DOX and } 15 \text { min BHF.", } \\
\text { "Old dummies can result in wafer } \\
\text { curvature." }\end{array}$ \\
\hline 6. & $\begin{array}{l}\text { Etching BHF (1:7) } \\
\mathrm{B}_{2} \mathbf{O}_{5} \\
(\# \text { etch026) }\end{array}$ & $\begin{array}{l}\text { CR112B / Wet-Bench 3-3 } \\
\mathrm{NH}_{4} \mathrm{~F} / \mathrm{HF}(1: 7) \text { VLSI: MERCK } 101171.2500 \\
\text { - Etch time: } 5 \text { min } \\
\text { - Quick Dump Rinse }<0.1 \mu \mathrm{S} \\
\text { - Spin drying }\end{array}$ & etch time: $15 \mathrm{~min}$ \\
\hline 7. & 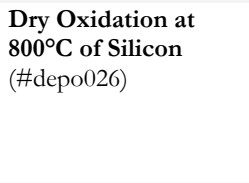 & $\begin{array}{l}\text { CR112B / Furnace B3 } \\
\text { Standby temperature: } 800{ }^{\circ} \mathrm{C} \\
\text { - Program: DOX- } 800 \\
\text { - Temp: } 800{ }^{\circ} \mathrm{C} \\
\text { - Gas: } \mathrm{O}_{2} \\
\text { - Flow: } 4 \mathrm{l} \cdot \mathrm{min}^{-1}\end{array}$ & oxidation time: $30 \mathrm{~min}$ \\
\hline 8. & $\begin{array}{l}\text { Etching BHF (1:7) } \\
\mathbf{B}_{2} \mathbf{O}_{5} \\
(\# \text { etch026) }\end{array}$ & $\begin{array}{l}\text { CR112B / Wet-Bench 3-3 } \\
\mathrm{NH}_{4} \mathrm{~F} / \mathrm{HF}(1: 7) \text { VLSI: MERCK } 101171.2500 \\
\text { - Etch time: } 5 \text { min } \\
\text { - Quick Dump Rinse }<0.1 \mu \mathrm{S} \\
\text { - Spin drying }\end{array}$ & etch time: $15 \mathrm{~min}$ \\
\hline 9. & $\begin{array}{l}\text { Sheet Resistance } \\
\text { measurement } \\
\text { (\#char009) }\end{array}$ & $\begin{array}{l}\text { CR118B / Resistance Measurement } \\
\text { Equipment }\end{array}$ & $\begin{array}{l}\text { sheet resistance } \pm 200 \mathrm{~nm} \\
\text { poly-Si' } \mathrm{i}^{++}: \\
\quad 453 \Omega \cdot \square^{-1}(1 \mathrm{hr} \mathrm{SSD}) \text {, } \\
\quad 815 \Omega \cdot \square^{-1}(45 \mathrm{~min} \mathrm{SSD}) \\
\text { " } R_{\text {sheet }}\left[\Omega \cdot \square^{-1}\right]=\mathrm{R}_{\text {meas }} \cdot \pi / \ln (2)= \\
\mathrm{R}_{\text {meas }} \cdot 4.532^{\text {" }}\end{array}$ \\
\hline 10. & $\begin{array}{l}\text { Lithography - } \\
\text { Priming (liquid) } \\
\text { (\#lith001) }\end{array}$ & $\begin{array}{l}\text { CR112B / Suss Micro Tech Spinner (Delta 20) } \\
\text { Hotplate } 120^{\circ} \mathrm{C} \\
\text { HexaMethylDiSilazane (HMDS) } \\
\text { - Dehydration bake }\left(120^{\circ} \mathrm{C}\right): 5 \mathrm{~min} \\
\text { - Spin program: } 4(4000 \mathrm{rpm}, 20 \mathrm{~s})\end{array}$ & \\
\hline 11. & $\begin{array}{l}\text { Lithography - } \\
\text { Coating Olin907-17 } \\
\text { (\#lith005) }\end{array}$ & $\begin{array}{l}\text { CR112B / Suss Micro Tech Spinner (Delta 20) } \\
\text { Hotplate } 95^{\circ} \mathrm{C} \\
\text { Olin } 907-17 \\
\text { - Spin Program: } 4(4000 \mathrm{rpm}, 20 \mathrm{~s}) \\
\text { - Prebake }\left(95^{\circ} \mathrm{C}\right): 90 \mathrm{~s}\end{array}$ & \\
\hline 12. & $\begin{array}{l}\text { Lithography - } \\
\text { Alignment \& } \\
\text { Exposure Olin 907-17 } \\
\text { (EV620) } \\
\text { (\#lith021) }\end{array}$ & $\begin{array}{l}\text { CR117B / EV620 } \\
\text { Electronic Vision Group } 620 \text { Mask Aligner } \\
\text { - Hg-lamp: } 12 \mathrm{~mW} \cdot \mathrm{cm}^{-2} \\
\text { - Exposure Time: } 4 \mathrm{~s}\end{array}$ & $\begin{array}{l}\text { mask: TCPY } \\
\text { exposure time: } 3.5 \mathrm{~s} \\
\text { mask: } 5^{"} 2.3 \mathrm{~mm} \\
\text { sub: } 4 " 0.53 \mathrm{~mm} \\
\text { contact: vacuum }\end{array}$ \\
\hline 13. & $\begin{array}{l}\text { Lithography - } \\
\text { Development Olin } \\
\text { Resist } \\
\text { (\#lith011) }\end{array}$ & $\begin{array}{l}\text { CR112B / Wet-Bench } \mathbf{1 1} \\
\text { Developer: OPD4262 } \\
\text { Hotplate } 120^{\circ} \mathrm{C}(\mathrm{CR} 112 \mathrm{~B} \text { or CR117B) } \\
\text { - After Exposure Bake }\left(120^{\circ} \mathrm{C}\right): 60 \mathrm{~s} \\
\text { Development: } \\
\text { - Time: } 30 \mathrm{~s} \text { in Beaker } 1 \\
\text { - Time: } 15-30 \mathrm{~s} \text { in Beaker } 2 \\
\text { - Quick Dump Rinse }<0.1 \mu \mathrm{S} \\
\text { - Spin drying }\end{array}$ & \\
\hline 14. & $\begin{array}{l}\text { Optical microscopic } \\
\text { inspection - } \\
\text { Lithography } \\
\text { (\#char001) }\end{array}$ & CR112B / Nikon Microscope & \\
\hline
\end{tabular}




\begin{tabular}{|c|c|c|c|}
\hline 15. & $\begin{array}{l}\text { Plasma etching - } \\
\text { chamber cleaning } \\
\text { (Etske) } \\
\text { (\# etch003) }\end{array}$ & $\begin{array}{l}\text { CR102A / Elektrotech PF310/340 } \\
\text { Select chamber for desired etch process } \\
\text { Select electrode for desired etch process } \\
\text { - Electrode temp.: } 10{ }^{\circ} \mathrm{C} \text { or } 25^{\circ} \mathrm{C} \\
\text { - O } \\
\text { - pressure: } 50 \mathrm{~m} \text { Torr } \\
\text { - power: } 150 \mathrm{~W} \\
\text { - DC-Bias: } 780 \mathrm{~V} \\
\text { "Chamber is clean when plasma color is white." }\end{array}$ & "Use quartz electrode." \\
\hline 16. & $\begin{array}{l}\text { Plasma etching } \\
\text { Silicon - Low etch- } \\
\text { rate (Etske) } \\
\text { (\#etch002) }\end{array}$ & $\begin{array}{l}\text { CR102A / Elektrotech PF310/340 } \\
\text { Clean chamber } \\
\text { Styros electrode } \\
\text { Showerhead } \\
\text { - Electrode temp.: } 10{ }^{\circ} \mathrm{C} \\
\text { - } \mathrm{SF}_{6} \text { flow: } 30 \mathrm{sccm} \\
\text { - } \mathrm{CHF}_{3} \text { flow: } 25 \mathrm{sccm} \\
\text { - } \mathrm{O}_{2} \text { flow: } 20 \mathrm{sccm} \\
\text { - pressure: } 75 \mathrm{mTorr} \\
\text { - DC-Bias: } 0-30 \mathrm{~V} \\
\text { - } 50 \mathrm{~W}: 300 \mathrm{~nm} \cdot \mathrm{min}^{-1} \\
\text { - } 60 \mathrm{~W}: 450 \mathrm{~nm} \cdot \mathrm{min}^{-1} \\
\text { - } 75 \mathrm{~W}: 530 \mathrm{~nm} \cdot \mathrm{min}^{-1} \\
\text { "valid for a } 3 \text { " wafer at } 50 \% \text { loading" }\end{array}$ & $\begin{array}{l}\text { power: } 50 \mathrm{~W} \\
\text { etch time: } 1 \mathrm{~min} 10 \mathrm{~s} \\
\text { "Topside and bottomside etching.", } \\
\text { "Protect topside during bottomside } \\
\text { etching with Oling907-17 resist.", } \\
\text { "Use quartz electrode." }\end{array}$ \\
\hline 17. & $\begin{array}{l}\text { Stripping of Olin PR - } \\
\text { standard } \\
\text { (\#lith016) }\end{array}$ & $\begin{array}{l}\text { CR112B / Wet-Bench 3-2 } \\
\mathrm{HNO}_{3}(100 \%) \text { Selectipur: MERCK } 100453 \\
\text { - Time: } 20 \mathrm{~min} \\
\text { - Quick Dump Rinse < }<.1 \mu \mathrm{S} \\
\text { - Spin drying } \\
\text { - Visual microscopic inspection }\end{array}$ & \\
\hline 18. & $\begin{array}{l}\text { Optical microscopic } \\
\text { inspection } \\
(\# \text { char002) }\end{array}$ & $\begin{array}{l}\text { CR112B / Nikon Microscope } \\
\text { CR117B / Olympus Microscope } \\
\text { CR102B / Olympus Microscope }\end{array}$ & \\
\hline 19. & $\begin{array}{l}\text { Surface profile } \\
\text { measurement } \\
\text { (\#char005) }\end{array}$ & CR118B / Veeco Dektak 8 & $\begin{array}{l}\text { "Measure layer tickness after BHF } \\
\text { etching.", "Measure wafer curvature } \\
\text { after SSD." }\end{array}$ \\
\hline 20. & $\begin{array}{l}\text { Cleaning Short } \\
\text { (\#clean002) }\end{array}$ & $\begin{array}{l}\text { CR112B / Wet-Bench } 131 \\
\mathrm{HNO}_{3}(100 \%) \text { Selectipur: MERCK } \\
\text { - Beaker 1: } \mathrm{HNO}_{3}(100 \%) 5 \mathrm{~min} \\
\text { - Beaker 2: } \mathrm{HNO}_{3}(100 \%) 5 \mathrm{~min} \\
\text { - Quick Dump Rinse <0.1 } \mu \mathrm{S} \\
\text { - Spin drying }\end{array}$ & \\
\hline 21. & $\begin{array}{l}\text { Sputtering of Al } \\
\text { (Oxford) } \\
\text { (\#depo022) }\end{array}$ & $\begin{array}{l}\text { CR106A / Oxford PL } 400 \\
\text { - Program: } 3000 \mathrm{~nm} \mathrm{Al} \text { pos } 1 \\
\text { - Pressure: } 10 \mathrm{~m} \text { Torr } \\
\text { Depositionrate (100 } \mathrm{mm} \text { wafer) : } 820 \mathrm{~nm} \cdot \mathrm{min}^{-1}\end{array}$ & $\begin{array}{l}\text { layer thickness: } 200 \mathrm{~nm} \\
\text { power: } 7 \mathrm{~kW} \\
\text { deposition time: } 15 \mathrm{~s}\end{array}$ \\
\hline 22. & $\begin{array}{l}\text { Lithography - } \\
\text { Priming (liquid) } \\
\text { (\#lith001) }\end{array}$ & $\begin{array}{l}\text { CR112B / Suss Micro Tech Spinner (Delta 20) } \\
\text { Hotplate } 120^{\circ} \mathrm{C} \\
\text { HexaMethylDiSilazane (HMDS) } \\
\text { - Dehydration bake }\left(120^{\circ} \mathrm{C}\right): 5 \mathrm{~min} \\
\text { - Spin program: } 4(4000 \mathrm{rpm}, 20 \mathrm{~s})\end{array}$ & \\
\hline 23. & $\begin{array}{l}\text { Lithography - } \\
\text { Coating Olin907-17 } \\
\text { (\#lith005) }\end{array}$ & $\begin{array}{l}\text { CR112B / Suss Micro Tech Spinner (Delta 20) } \\
\text { Hotplate } 95^{\circ} \mathrm{C} \\
\text { Olin } 907-17 \\
\text { - Spin Program: } 4(4000 \mathrm{rpm}, 20 \mathrm{~s}) \\
\text { - Prebake }\left(95^{\circ} \mathrm{C}\right): 90 \mathrm{~s}\end{array}$ & \\
\hline 24. & $\begin{array}{l}\text { Lithography - } \\
\text { Alignment \& } \\
\text { Exposure Olin 907-17 } \\
\text { (EV620) } \\
\text { (\#lith021) }\end{array}$ & $\begin{array}{l}\text { CR117B / EV620 } \\
\text { Electronic Vision Group } 620 \text { Mask Aligner } \\
\text { - Hg-lamp: } 12 \mathrm{~mW} \cdot \mathrm{cm}^{-2} \\
\text { - Exposure Time: } 4 \mathrm{~s}\end{array}$ & $\begin{array}{l}\text { mask: TCAL } \\
\text { exposure time: } 3.5 \mathrm{~s} \\
\text { mask: } 5 " 2.3 \mathrm{~mm} \\
\text { sub: } 4 \text { " } 0.53 \mathrm{~mm} \\
\text { contact: vacuum }\end{array}$ \\
\hline 25. & $\begin{array}{l}\text { Lithography - } \\
\text { Development Olin } \\
\text { Resist }\end{array}$ & $\begin{array}{l}\text { CR112B / Wet-Bench } \mathbf{1 1} \\
\text { Developer: OPD } 4262 \\
\text { Hotplate } 120^{\circ} \mathrm{C}(\mathrm{CR} 112 \mathrm{~B} \text { or CR117B) }\end{array}$ & \\
\hline
\end{tabular}




\begin{tabular}{|c|c|c|c|}
\hline & (\#lith011) & $\begin{array}{l}\text { - After Exposure Bake }\left(120^{\circ} \mathrm{C}\right): 60 \mathrm{~s} \\
\text { Development: } \\
\text { - Time: } 30 \mathrm{~s} \text { in Beaker } 1 \\
\text { - Time: } 15-30 \mathrm{~s} \text { in Beaker } 2 \\
\text { - Quick Dump Rinse <0.1 } \mu \mathrm{S} \\
\text { - Spin drying }\end{array}$ & \\
\hline 26. & $\begin{array}{l}\text { Optical microscopic } \\
\text { inspection - } \\
\text { Lithography } \\
\text { (\#char001) }\end{array}$ & CR112B / Nikon Microscope & \\
\hline 27. & $\begin{array}{l}\text { Etching of Al Wet } \\
\text { (\# } \# \text { etch035) }\end{array}$ & $\begin{array}{l}\text { CR116B / Wet-Bench } 2 \\
\text { Aluminium etch: MERCK } 115435.2500 \\
\text { - Temp.: } 55^{\circ} \mathrm{C} \\
\text { - Quick Dump Rinse }<0.1 \mu \mathrm{S} \\
\text { - Spin drying } \\
\text { Etchrates: } 1 \mu \mathrm{m} \cdot \mathrm{min}^{-1}\end{array}$ & $\begin{array}{l}\text { temp: } 40^{\circ} \mathrm{C} \\
\text { etch time: } 40 \mathrm{~s} \\
\text { "Prepare to quickly stop the etching } \\
\text { process in a beaker filled with DI } \\
\text { water." }\end{array}$ \\
\hline 28. & $\begin{array}{l}\text { Stripping of polymers } \\
\text { in } \mathrm{HNO}_{3} \text { multi- } \\
\text { purpose } \\
\text { (\#lith014) }\end{array}$ & $\begin{array}{l}\text { CR116B / Wet-Bench } 2 \\
\mathrm{HNO}_{3}(100 \%) \text { Selectipur: MERCK } 100453 \\
\text { - Beaker 1: } \mathrm{HNO}_{3}(100 \%) 20 \mathrm{~min} \\
\text { - Quick Dump Rinse <0.1 } \mu \mathrm{S} \\
\text { - Spin drying }\end{array}$ & \\
\hline 29. & $\begin{array}{l}\text { Optical microscopic } \\
\text { inspection } \\
(\# \text { char002) }\end{array}$ & $\begin{array}{l}\text { CR112B / Nikon Microscope } \\
\text { CR117B / Olympus Microscope } \\
\text { CR102B / Olympus Microscope }\end{array}$ & \\
\hline
\end{tabular}

\section{A.3 Surface Microchannel $\mathrm{SF}_{6}$ Plasma Release}

\begin{tabular}{|c|c|c|c|}
\hline Nr. & Process & Process Parameters & $\begin{array}{c}\text { Design related parameters } \\
\text { and remarks }\end{array}$ \\
\hline 1. & $\begin{array}{l}\text { Lithography - } \\
\text { Priming (liquid) } \\
\text { (\#lith001) }\end{array}$ & $\begin{array}{l}\text { CR112B / Suss Micro Tech Spinner (Delta 20) } \\
\text { Hotplate } 120^{\circ} \mathrm{C} \\
\text { HexaMethylDiSilazane (HMDS) } \\
\text { - Dehydration bake }\left(120^{\circ} \mathrm{C}\right): 5 \mathrm{~min} \\
\text { - Spin program: } 4(4000 \mathrm{rpm}, 20 \mathrm{~s})\end{array}$ & \\
\hline 2. & $\begin{array}{l}\text { Lithography - } \\
\text { Coating Olin908-35 } \\
\text { (\#lith006) }\end{array}$ & $\begin{array}{l}\text { CR112B / Suss Micro Tech Spinner (Delta 20) } \\
\text { Hotplate } 95^{\circ} \mathrm{C} \\
\text { Olin } 908-35 \\
\text { - Spin Program: } 4(4000 \mathrm{rpm}, 20 \mathrm{~s}) \\
\text { - Prebake }\left(95^{\circ} \mathrm{C}\right): 120 \mathrm{~s}\end{array}$ & $\begin{array}{l}\text { spin speed: } 2000 \mathrm{rpm} \\
\text { layer thickness: } 5 \mu \mathrm{m} \\
\text { "Dry wafer edge beads for } 1 \mathrm{hr} \text { in } \\
\text { yellow room." }\end{array}$ \\
\hline 3. & $\begin{array}{l}\text { Lithography - } \\
\text { Alignment \& } \\
\text { Exposure Olin 907-17 } \\
\text { (EV620) } \\
\text { (\#lith021) }\end{array}$ & $\begin{array}{l}\text { CR117B / EV620 } \\
\text { Electronic Vision Group } 620 \text { Mask Aligner } \\
\text { - Hg-lamp: } 12 \mathrm{~mW} \cdot \mathrm{cm}^{-2} \\
\text { - Exposure Time: } 4 \mathrm{~s}\end{array}$ & $\begin{array}{l}\text { mask: RLSE } \\
\text { exposure time: } 22 \mathrm{~s} \\
\text { contact: soft }\end{array}$ \\
\hline 4. & $\begin{array}{l}\text { Lithography - } \\
\text { Development Olin } \\
\text { Resist } \\
\text { (\#lith011) }\end{array}$ & $\begin{array}{l}\text { CR112B / Wet-Bench } \mathbf{1 1} \\
\text { Developer: OPD4262 } \\
\text { Hotplate } 120^{\circ} \mathrm{C}(\mathrm{CR} 112 \mathrm{~B} \text { or CR117B) } \\
\text { - After Exposure Bake }\left(120^{\circ} \mathrm{C}\right): 60 \mathrm{~s} \\
\text { Development: } \\
\text { - Time: } 30 \mathrm{~s} \text { in Beaker } 1 \\
\text { - Time: } 15-30 \mathrm{~s} \text { in Beaker } 2 \\
\text { - Quick Dump Rinse }<0.1 \mu \mathrm{S} \\
\text { - Spin drying }\end{array}$ & $\begin{array}{l}\text { bkr1 develop time: } 1 \mathrm{~min} \\
\text { bkr2 develop time: } 30 \mathrm{~s} \\
\text { "No after exposure bake, avoiding } \\
\text { cracking of the resist layer." }\end{array}$ \\
\hline 5. & $\begin{array}{l}\text { Optical microscopic } \\
\text { inspection - } \\
\text { Lithography } \\
\text { (\#char001) }\end{array}$ & CR112B / Nikon Microscope & \\
\hline 6. & $\begin{array}{l}\text { Lithography - } \\
\text { Postbake standard } \\
\text { (\#lith009) }\end{array}$ & $\begin{array}{l}\text { CR112B / Hotplate } 120^{\circ} \mathbf{C} \\
\text { - Time: } 30 \mathrm{~min}\end{array}$ & \\
\hline
\end{tabular}




\begin{tabular}{|c|c|c|c|c|}
\hline 7. & $\begin{array}{l}\text { Plasma etching - } \\
\text { chamber cleaning } \\
\text { (Etske) } \\
\text { (\#etch003) }\end{array}$ & \multicolumn{2}{|c|}{$\begin{array}{l}\text { CR102A / Elektrotech PF310/340 } \\
\text { Select chamber for desired etch process } \\
\text { Select electrode for desired etch process } \\
\text { - Electrode temp.: } 10^{\circ} \mathrm{C} \text { or } 25^{\circ} \mathrm{C} \\
\text { - } \mathrm{O}_{2} \text { flow: } 20 \mathrm{sccm} \\
\text { - pressure: } 50 \mathrm{mTorr} \\
\text { - power: } 150 \mathrm{~W} \\
\text { - DC-Bias: } 780 \mathrm{~V} \\
\text { "Chamber is clean when plasma color is white." }\end{array}$} & "Use quartz electrode." \\
\hline 8. & $\begin{array}{l}\text { Plasma etching SiN } \\
\text { (Etske) } \\
\text { (\#etch004) }\end{array}$ & \multicolumn{2}{|c|}{$\begin{array}{l}\text { CR102A / Elektrotech PF310/340 } \\
\text { Dirty chamber } \\
\text { Styros electrode } \\
\text { - Electrode temp.: } 10{ }^{\circ} \mathrm{C} \\
\text { - } \mathrm{CHF}_{3} \text { flow: } 25 \mathrm{sccm} \\
\text { - } \mathrm{O}_{2} \text { flow: } 5 \mathrm{sccm} \\
\text { - pressure: } 10 \mathrm{mTorr} \\
\text { - power: } 75 \mathrm{~W} \\
\text { Etchrate } \mathrm{SiN}: 50 \mathrm{~nm} \cdot \mathrm{min}^{-1}\left(\text { for } \mathrm{V}_{\mathrm{DC}}=-460 \mathrm{~V}\right) \\
\text { Etchrate } \mathrm{SiN}: 75 \mathrm{~nm} \cdot \mathrm{min}^{-1}\left(\text { for } \mathrm{V}_{\mathrm{DC}}=-580 \mathrm{~V}\right) \\
\text { Etchrate Olin resist : } 95 \mathrm{~nm} \cdot \mathrm{min}^{-1} \\
\text { "IfDC-Bias }<375 \mathrm{~V} \text { apply chamber clean }(\# \text { tetch003)." }\end{array}$} & $\begin{array}{l}\text { nom. etching rate: } 60 \mathrm{~nm} \cdot \mathrm{min}^{-1} \\
\text { layer thickness: } 1.8 \mu \mathrm{m} \\
\text { nom. etch time: } 33 \mathrm{~min} \\
\text { "Use quartz electrode." }\end{array}$ \\
\hline \multirow[t]{10}{*}{9.} & \multirow{10}{*}{$\begin{array}{l}\text { Plasma etching } \\
\text { Silicon SCT release } \\
\text { (\# \#tch076) }\end{array}$} & \multicolumn{2}{|c|}{$\begin{array}{l}\text { CR 125c/Adixen AMS } 100 \mathrm{SE} \\
\text { Applications: release channels (SCT) }\end{array}$} & \multirow{10}{*}{$\begin{array}{l}\text { nom. etch rate: } 11 \mu \mathrm{m} \cdot \mathrm{min}^{-1} \\
\text { etch time: } 20 \mathrm{~min} \\
\text { nom. etch depth: } 220 \mu \mathrm{m}\end{array}$} \\
\hline & & Parameters & Value & \\
\hline & & $\mathrm{SF}_{6}[\mathrm{sccm}]$ & 350 & \\
\hline & & He-backside cooling [mbar] & 10 & \\
\hline & & ICP [W] & 1800 & \\
\hline & & CCP On/Off [ms] & 0 & \\
\hline & & $\mathrm{p}[\mathrm{Pa}]$ & 10 & \\
\hline & & APC position $\%$ & 15 & \\
\hline & & Electrode temperature $\left[{ }^{\circ} \mathrm{C}\right]$ & 0 & \\
\hline & & Substrate height $[\mathrm{mm}]$ & 200 & \\
\hline 10. & $\begin{array}{l}\text { Plasma etching SiN } \\
\text { (Etske) } \\
\text { (\# etch004) }\end{array}$ & \multicolumn{2}{|c|}{$\begin{array}{l}\text { CR102A / Elektrotech PF310/340 } \\
\text { Dirty chamber } \\
\text { Styros electrode } \\
\text { - Electrode temp.: } 10{ }^{\circ} \mathrm{C} \\
\text { - } \mathrm{CHF}_{3} \text { flow: } 25 \mathrm{sccm} \\
\text { - } \mathrm{O}_{2} \text { flow: } 5 \mathrm{sccm} \\
\text { - pressure: } 10 \mathrm{mTorr} \\
\text { - power: } 75 \mathrm{~W} \\
\text { Etchrate } \mathrm{SiN}: 50 \mathrm{~nm} \cdot \mathrm{min}^{-1}\left(\text { for } \mathrm{V}_{\mathrm{DC}}=-460 \mathrm{~V}\right) \\
\left.\text { Etchrate } \mathrm{SiN}: 75 \mathrm{~nm} \cdot \mathrm{min}^{-1} \text { (for } \mathrm{V}_{\mathrm{DC}}=-580 \mathrm{~V}\right) \\
\text { Etchrate Olin resist: } 95 \mathrm{~nm} \cdot \mathrm{min}^{-1} \\
\text { "IfDC-Bias }<375 \mathrm{~V} \text { apply chamber clean (\# } \text { etch003)." }\end{array}$} & $\begin{array}{l}\text { nom. etching rate: } 60 \mathrm{~nm} \cdot \mathrm{min}^{-1} \\
\text { nom. etch time: } 7 \mathrm{~min} \\
\text { "Use quartz electrode.", "Open inlet } \\
\text { holes to the surface microchannels." }\end{array}$ \\
\hline 11. & $\begin{array}{l}\text { Stripping of polymers } \\
\text { in } \mathrm{HNO}_{3} \text { multi- } \\
\text { purpose } \\
\text { (\#lith014) }\end{array}$ & \multicolumn{2}{|c|}{$\begin{array}{l}\text { CR116B / Wet-Bench } 2 \\
\mathrm{HNO}_{3}(100 \%) \text { Selectipur: MERCK } 100453 \\
\text { - Beaker 1: } \mathrm{HNO}_{3}(100 \%) 20 \mathrm{~min} \\
\text { - Quick Dump Rinse <0.1 } \mu \mathrm{S} \\
\text { - Spin drying }\end{array}$} & \\
\hline 12. & $\begin{array}{l}\text { Optical microscopic } \\
\text { inspection } \\
(\# \text { char002) }\end{array}$ & \multicolumn{2}{|c|}{$\begin{array}{l}\text { CR112B / Nikon Microscope } \\
\text { CR117B / Olympus Microscope } \\
\text { CR102B / Olympus Microscope }\end{array}$} & \\
\hline
\end{tabular}




\section{Summary}

The emerging fields of micro total-analysis systems (micro-TAS), micro-reactors and bio-MEMS drives the need for further miniaturisation of sensors measuring quantities such as pressure, temperature and flow. The research described in this thesis concerns the development of low-drift micro flow sensors for accurate measurement of minute amount of liquid flow in the $\mathrm{nl} \cdot \mathrm{min}^{-1}$ range.

Miniaturisation means that flow channel dimensions and flow rates become smaller. This requires thermally-isolated flow channels, where the complete fluid can be heated in order to obtain maximum sensitivity. A microchannel fabrication concept (Chap. 3) was developed, based on buried channel technology (BCT), allowing for easy fluidic interfacing and integration of transducer materials in close proximity to the fluid. This is achieved by the reliable fabrication of completely sealed microchannels directly below the substrate surface. The channel technology has found application in the fabrication of resonant flow sensors and low-drift micro flow sensors (Chap. 4-8) in the $\mathrm{nl} \cdot \mathrm{min}^{-1}$ range. Additionally, the technology has been extended by the possibility to integrate nanochannels using fluidic vias. This has been used in the fabrication of nano-nozzle electrospray emitters (Chap. 9).

In current micromachined thermal flow sensors the elements for temperature sensing are made by thin films. The problem is that thin films reproduce poorly and that practically all materials properties are subject to drift. This translates directly into the accuracy of thermal micro flow sensors. In this thesis low-drift micro flow sensors were investigated, using two heaters and a thermopile in order to eliminate material drift (Chap. 5). The low offset drift of thermopiles has been exploited in a feedback loop controlling the dissipated powers in the heater resistors, minimising inevitable influences of resistance drift, mismatch of thin-film metal resistors and thermopile material drift. The control system cancels the flow-induced temperature difference across the thermopile by controlling a power difference between both heater resistors, thereby giving a measure of the flow rate. Alternatively, a sensor resistor and heat waves can be used to provide for a low offset-drift error signal (Chap. 8). It was demonstrated that material drift can largely be compensated.

Sensitivity can be increased by designing flow sensors with a large number of integrated thermocouple junctions (Chap. 6), however it was observed that externally applied temperature gradients over the chip can still lead to drift of the sensor output signal. A special meandering microchannel layout was used to create a fully symmetrical flow sensor, where the arrangement of thermopile junctions has resulted in low-drift micro thermal flow sensors for liquids in the $\mathrm{nl} \cdot \mathrm{min}^{-1}$ range, with compensation for external temperature gradients (Chap. 7). 


\section{Samenvatting}

De opkomst van micro-total-analysis-systems (micro-TAS), micro-reactoren en bio-MEMS genereert de behoefte tot verdere miniaturisering van sensoren voor grootheden als druk, temperatuur en stromingssnelheid. Het onderzoek dat in dit proefschrift wordt beschreven, betreft de ontwikkeling van lage-drift microstromingssnelheidssensoren voor het nauwkeurig meten van minuscule vloeistofstroomsnelheden in het $\mathrm{nl} \cdot \mathrm{min}^{-1}$ bereik.

Miniaturisering betekent dat de afmetingen van de vloeistofkanalen en de daarin optredende stroomsnelheden afnemen. Hierdoor wordt het noodzakelijk om de kanalen thermisch te isoleren, om zo de volledige vloeistof in het kanaal te kunnen verwarmen, waarbij een maximale gevoeligheid kan worden verkregen. Hiertoe is een fabricageconcept ontwikkeld voor microkanalen (Hfst. 3), gebaseerd op burriedchannel-technologie (BCT). Deze kanalen zijn eenvoudig fluïdisch aan te sluiten, waarbij transducenten geïntegreerd kunnen worden dicht bij de vloeistof. Dit wordt bereikt door fabricage van volledig gesloten microkanalen direct onder het oppervlak van het substraat. Deze technologie vindt toepassing in resonerende stromingssnelheidssensoren en lage-drift, thermische stromingssnelheidssensoren. (Hfst. 4-8). Daarbij is de technolgie verder uitgebreid met de mogelijkheid om nanokanalen via fluïdische verbindingen te integreren met microkanalen. Dit is gebruikt bij de fabricage van nano-nozzle-electrospray-emitters (Hfst. 9).

In huidige micro-stromingssnelheidssensoren bestaan de elementen voor het meten van de temperatuur uit dunne films. Het probleem is dat bijna alle dunne films moeilijk reproduceerbaar zijn en dat de materiaaleigenschappen onderhevig zijn aan drift, hetgeen de nauwkeurigheid van de sensoren beïnvloedt. In dit proefschrift worden lage-driftsensoren, bestaande uit twee verwarmingselementen en een thermozuil, onderzocht om de invloed van drift in materiaaleigenschappen te elimineren (Hfst. 5). De lage offsetdrift van thermozuilen wordt gebruikt om de invloed van weerstandsdrift en ongelijkheid van weerstandswaarden en drift in de thermozuilmaterialen te minimaliseren d.m.v. een terugkoppellus, waarbij het gedissipeerde vermogen in de verwarmingselementen wordt bijgeregeld. Het regelsysteem regelt het temperatuurverschil t.g.v. de vloeistofstroming naar nul, door het verschil in gedissipeerde vermogen van de verwarmingsweerstanden aan te passen. Het benodigde vermogensverschil is daarbij een maat voor de stromingssnelheid. Een andere methode maakt gebruik van warmtegolven om een lage-drift foutsignaal te verkrijgen (Hfst. 8). Het is aangetoond dat materiaaldrift hiermee voor een groot deel wordt gecompenseerd. 
Een grotere gevoeligheid kan verkregen worden door een groot aantal thermokoppeljuncties te gebruiken (Hfst. 6). Echter, extern aangelegde temperatuurverschillen over de chip kunnen nog steeds leiden tot drift van het uitgangssignaal. Een speciale, meanderende kanaalstructuur kan gebruikt worden om een volledig symmetrische sensor te verkrijgen, resulterende in lage-drift micro- thermischestromingssnelheidssensoren voor vloeistofstroomsnelheden in het $\mathrm{nl} \cdot \mathrm{min}^{-1}$ bereik, met compensatie voor extern aangelegde temperatuurverschillen (Hfst. 7). 


\section{List of Publications}

\section{J ournal Articles}

\section{In preparation and submitted:}

- M. Dijkstra, T.S.J. Lammerink, M.J. de Boer, J.W. Berenschot, R.J. Wiegerink, M.C. Elwenspoek, "Low-drift flow sensor with thermopile-based power feedback", J. Micromech. S., (accepted for publication).

- M. Dijkstra, N.R. Tas, J.W. Berenschot, T. Hankemeier, M.J. de Boer, T.S.J. Lammerink, R.J. Wiegerink, M. Elwenspoek, "Nano-nozzle electrospray emitters fabricated by a micro- to nano-fluidic via technology", Lab Chip, (to be submitted).

- M. Dijkstra, T.S.J. Lammerink, M.J. de Boer, R.J. Wiegerink, M. Elwenspoek, "Ambient temperature-gradient compensated low-drift thermopile flow sensor", Sensor. Actuat. A-Phys., (in preparation).

- M. Dijkstra, T.S.J. Lammerink, O. Pjetri, M.J. de Boer, J.W. Berenschot, R.J. Wiegerink, M. Elwenspoek, "AC-driven temperature-balancing flow sensor with power feedback", J. Micromech. Microeng., (in preparation).

\section{Published:}

M. Dijkstra, M.J. de Boer, J.W. Berenschot, T.S.J. Lammerink, R.J. Wiegerink, M. Elwenspoek, "Miniaturized thermal flow sensor with planar integrated sensor structures on semicircular surface channels", Sensor. Actuat. A-Phys., 143 (2008) 1-6.

- M. Dijkstra, M.J. de Boer, J.W. Berenschot, T.S.J. Lammerink, R.J. Wiegerink, M. Elwenspoek, "A versatile surface channel concept for microfluidic applications", J. Micromech. Microeng., 17 (2007) 1971-1977.

- M. Dijkstra, J.J. van Baar, R.J. Wiegerink, T.S.J. Lammerink, J.H. de Boer, G.J.M. Krijnen, “Artificial sensory hairs based on the flow sensitive receptor hairs of crickets, J. Micromech. Microeng., 15 (2005) S132-S138.

- J.W. van Honschoten, D.R. Yntema, V. Svetovoy, M. Dijkstra, R.J. Wiegerink, M.C. Elwenspoek, "Analysis of the performance of a particle velocity sensor between two cylindrical obstructions", J. Acoust. Soc. Am., 121 (2007) 2711-2722.

- G.J.M. Krijnen, M. Dijkstra, J.J. van Baar, S.S. Shankar, W.J. Kuipers, J.H. de Boer, D. Altpeter, T.S.J. Lammerink, R.J. Wiegerink, "MEMS based hair flow-sensors as model systems for acoustic perception studies", Nanotechnology, 17 (2006) S84-S89.

\section{Conference Contributions}

- M. Dijkstra, T.S.J. Lammerink, M.J. de Boer, R.J. Wiegerink, M. Elwenspoek, "Ambient temperature-gradient compensated low-drift thermopile flow sensor", Proc. IEEE MEMS, (2009) 479-482.

- M. Dijkstra, T.S.J. Lammerink, O. Pjetri, M.J. de Boer, J.W. Berenschot, R.J. Wiegerink, M. Elwenspoek, “AC-driven temperature-balancing flow sensor with zero-offset power feedback”, Proc. MME, (2008). 
- M. Dijkstra, T.S.J. Lammerink, M.J. de Boer, J.W. Berenschot, R.J. Wiegerink, M.C. Elwenspoek, "Low-drift U-shaped thermopile flow sensor", Proc. IEEE Sensors, (2008).

- M. Dijkstra, N.R. Tas, J.W. Berenschot, M.J. de Boer, T.S.J. Lammerink, R.J. Wiegerink, M.C. Elwenspoek, "Micro-Fluidic to nano-fluidic via technology with application to nano-electrospray", Proc. MNE, (2008).

- M. Dijkstra, T.S.J. Lammerink, M.J. de Boer, J.W. Berenschot, R.J. Wiegerink, M. Elwenspoek, "Low-drift flow sensor with zero-offset thermopile-based power feedback", Proc. DTIP, (2008).

- M. Dijkstra, M.J. de Boer, J.W. Berenschot, T.S.J. Lammerink, R.J. Wiegerink, M. Elwenspoek, "Miniaturized flow sensor with planar integrated sensor structures on semicircular surface channels", Proc. IEEE MEMS, (2007).

- M. Dijkstra, M.J. de Boer, J.W. Berenschot, T.S.J. Lammerink, R.J. Wiegerink, M. Elwenspoek, "Versatile surface channel concept for microfluidic applications", Proc. MME, (2006).

- $\quad$ M. Dijkstra, T.S.J. Lammerink, R.J. Wiegerink, M. Elwenspoek, "Nano-flow thermal sensors applying dynamic $\omega-2 \omega$ sensing method", Proc. MME, (2006) 29-32.

- M. Dijkstra, J.J. van Baar, R.J. Wiegerink, T.S.J. Lammerink, J.H. de Boer, G.J.M. Krijnen, "Capacitive flow sensors based on receptor hairs of crickets", Proc. MME, (2004).

- J. Haneveld, T.S.J. Lammerink, M. Dijkstra, H. Droogendijk, M.J. de Boer, R.J. Wiegerink, "Highly sensitive micro Coriolis mass flow sensor", Proc. IEEE MEMS (2008).

- $\quad$ G.J.M. Krijnen, J. Floris, M. Dijkstra, T.S.J. Lammerink, R.J. Wiegerink, "Biomimetic micromechanical adaptive flow-sensor array", Proc. SPIE Europe, (2007).

- J.W. van Honschoten, D.R. Yntema, M. Dijkstra, V. Svetovoy, R.J. Wiegerink, M.C. Elwenspoek, "Analysis of packaging effect on the performance of the Microflown", Proc. DTIP, (2006).

- G.J.M. Krijnen, M. Dijkstra, J.J. van Baar, T.S.J. Lammerink, R.J. Wiegerink, "Biomimetic, distributed micromechanical flow-sensing", Proc. Eurosensors, (2006).

S. Saravanan, M. Dijkstra, E. Berenschot, G. Krijnen, M. Elwenspoek “AlN thin film unimorph piezoelectric actuators on polysilicon microbridges", Proc. MME, (2005).

- J.J.van Baar, M. Dijkstra, R.J. Wiegerink, T.S.J. Lammerink, J.H. de Boer, G.J.M. Krijnen, "Arrays of cricket-inspired sensory hairs with capacitive motion detection", Proc. IEEE MEMS, (2005).

- J.J. van Baar, J.H. de Boer, M. Dijkstra, R.J. Wiegerink, T.S.J. Lammerink, G.J.M. Krijnen, "Fabrication of artificial hairs with capacitive read-out for flow sensing", Proc. MME, (2004).

- J.J. van Baar, M. Dijkstra, R.J. Wiegerink, T.S.J. Lammerink, G.J.M. Krijnen, "Fabrication of arrays of artificial hairs for complex flow pattern recognition", Proc. IEEE Sensors, (2003).

- J.J. van Baar., W.A. Verweij, M. Dijkstra, R.J. Wiegerink, T.S.J. Lammerink, G.J.M. Krijnen, "Micromachined two dimensional resistor arrays for determination of gas parameters”, Proc. IEEE Tranducers (2003) 1606-1609. 


\section{Dankwoord}

$\mathrm{Na}$ vier jaar onderzoek is het dan gelukt dit proefschrift uit, grotendeels, publicaties samen te stellen. Vaak was de ene sensor net af of er waren voldoende nieuwe ideeën om een nog betere sensor te maken. Theo, bedankt dat wij het, soms met de nodige stevige discussies, zo ver hebben weten te brengen. Jammer, dat resultaten soms belangrijker leken, dan het bieden van ondersteuning bij het verrichten van mijn onderzoek. Tijdens het schrijven van mijn proefschrift heeft het willen doorgronden van driftinvloeden mij daarom nog de nodige hoofdbrekens gekost. Nu kan ik dit voldoende begrijpen. Remco W., bedankt voor je bijdrage aan mijn onderzoek en dat je mij de laatste maanden hebt ondersteund in het voltooien van dit proefschrift.

Ik herinner mij de colleges electrostatica en -dynamica van Miko. De sturende manier van onderwijs en tegelijk ongedwongen stimulans om de materie jezelf eigen te maken, spraken mij zeer aan. Iets wat later als onderwijsassistent, samen met Leon en Miko, binnen probleemgestuurd onderwijs niet zo makkelijk is om zelf toe te passen. De studenten de collegestof bij te brengen, door met opgaven te helpen, heb ik, onder andere met Johan, Wabe en Iris, met toenemend plezier gedaan.

Gijs, bedankt dat ik na mijn afstudeeropdracht een tijdje als medewerker-onderzoek door heb kunnen werken aan de uitlezing van kunstmatige krekelharen. Dit was natuurlijk niet mogelijk geweest zonder de haren van Rik, die deze met toewijding uit de cleanroom heeft getrokken. Bedankt John voor de samenwerking en dankzij jou kan ik nu ook een beetje jongleren. Daarnaast was er altijd wel een leuk gesprek met Toon en Saravanan als we in de mensa aten. Voor de nodige intellectuele ontspanning werden er af en toe met Joost algemene relativiteitspuzzels opgelost. "De covariante afgeleide van een contravariant tensorveld gegeven een affiene connectie" te ontrafelen is toch iets voor de meer gevorderde puzzelaar.

Meint en Erwin zijn wat mij betreft de beste werkpaarden van stal. Het was snel duidelijk dat Meint de kar eerder had getrokken en binnen afzienbare tijd hebben we een microkanaal-technologie neergezet, die mij de mogelijkheid gaf om de nodige sensoren te realiseren. Gaandeweg heb ik veel kunnen leren van Erwin z'n inzicht in cleanroom-technologie, al bleef het af en toe flink zweten in de cleanroom. In het algemeen verliep de fabricage van de sensoren echter zonder problemen, mede dankzij de $\mathrm{MESA}^{+}$cleanroom-medewerkers Eddy, Hans, Huib, Ite-Jan, Marion, Rene en Samantha.

Niels, bedankt voor het idee om de fluïdische via-technologie te gebruiken voor electrospray-experimenten en je motivatie om er een artikel van te maken. Ook Johnny wil ik bedanken voor zijn hulp met de FIB. Remco S., je suggesties met betrekking tot de meetopstelling en fluïdishe aansluitingen waren altijd nuttig om ver- 
der te komen. Olti, je inzet en goede samenwerking in het verrichten van de metingen voor Hfst. 8 worden zeer gewaardeerd. De posterprijs op de MME 2008-conferentie in Aachen was en mooie beloning voor je werk.

Ook andere conferenties waren voor mij de moeite waard. Daarbij wil ik Edin graag bedanken als onze gids in Tokyo. Ik heb nooit geweten dat Japanners zo goed kunnen dansen. Johan en zijn vrienden van IBM Zürich wil ik daarnaast bedanken voor het adhoc regelen van een leuke rondreis in Griekenland na de MNE 2009conferentie in Athene. Alle overige mensen van de TST-afdeling, waarmee ik samengewerkt heb, Ahmad, Arjan, Bas, Berker, Boudewijn, Chris, Dannis, Deladi, Dick, Doekle, Duy, Hadi, Hammad, Hanh, Harmen, Henk, Henry, Hien, Imran, Ingrid, Jeroen, Judith, Kees, Laura, Luis, Marcel K., Marcus, Maryana, Mink, Mubassira, Narges, Nataliya, Paul, Ram, Roald, Robert, Sandeep, Satya, Shahina, Susan, Vitaly, Wilco, Willem, Winfred, Yiping et al., wil ik bedanken voor de prettige werksfeer, de vele slappe bakken tijdens de koffie, de leuke borrels en uitjes en voor het feit dat uiteindelijk iedereen z'n eigen gang kan gaan.

Srinivas, ik heb jou leren kennen als iemand die zich goed kan concentreren op zijn werk en daaraan richting weet te geven. Als huisgenoot was je bovendien vindingrijk in het uitzoeken van reisjes door Europa en ander activiteiten. Ons verblijf in Londen en de reis door Italië met Nima waren erg leuk. Daarnaast heb ik veel plezier gehad aan de cricket-wedstrijden, ondanks de vele blauwe plekken. Was je er niet, dan had ik nog altijd Dirandhra als "temporary Indian housemate". Mijn vrienden Jan en Sam wil ik bedanken voor de vele gezellige avonden en de zomervakanties, die wij samen met Eelke hebben ondernomen.

Van stekkerdozen en schakelborden werd ik op jonge leeftijd voorzien door opa Dijkstra, die een bruin/witgoed- en installatiezaak in Loppersum had. Daarnaast waren er de inspirerende verhalen over mijn grootvader Geert de Vries, die op jonge leeftijd een constructiebedriif begon. Op de rand van een oude krant werden ideeën uitgewerkt en indien nodig laste hij mee als een grote klus af moest. Ik bedank oma de Vries dat altijd alles kon, ook als sommige projecten van mij enigzins uit de hand dreigden te lopen. Tenslotte wil ik mijn paps \& moes bedanken voor hun liefdevolle steun in de afgelopen jaren.

Marcel Dijkstra

Enschede, 23 maart 2009 


\section{Biography}

Marcel Dijkstra was born in Groningen, The Netherlands on the $28^{\text {th }}$ of april 1978. He went through primary school and MAVO in Loppersum, The Netherlands till 1994. Subsequently, the HAVO/MBO diploma was obtained in 1996 at the Eemsmondcollege in Delfziil, The Netherlands. In the same year he started his study to become an electrical engineer at the Hanzehogeschool in Groningen, The Netherlands. The first internship was followed at Philips Semiconductors in Nijmegen, The Netherlands at the Quality \& Reliability department. Electromigration measurements on aluminium interconnects and degradation measurements on transistors were conducted. Additionally, work was continued on statistical analysis software for the processing of reliability data. A second internship was conducted with Albert Martena at the University Medical Centre Groningen, The Netherlands. Work was done on measurement software for the determination of motor abilities in patients with Parkinson's disease. Activities included the implementation of fastfourier-transform algorithms in assembler and the implementation of wavelet-transform algorithms. The final bachelor assignment dealt with the development of a radio-controlled robot car using a prototype Bluetooth chip and was conducted together with Albert Martena at the university of Karlskrona/Ronneby, Sweden. Their examination work was awarded the Telecomcity - Sigma Exallon Systems prize in the province of Blekinge, Sweden in 2000. Work was continued for a few months on a prototype Bluetooth extension microphone for a conference telephone from Konftel AB in Umeå, Sweden. Study was continued at the University of Twente, were he obtained the M.Sc. degree in electrical engineering in 2004. The masters project was conducted at the Transducers Science \& Technology group and dealt with the development of artificial sensory hairs based on crickets hairs. He worked on that project as temporary research associate until $2006 . \mathrm{He}$ then started as a Ph.D. student on the research described in this thesis.

\section{Biografie}

Marcel Dijkstra werd geboren op 28 april 1978 te Groningen. Hij doorliep de lagere school en MAVO in Loppersum tot 1994. Daarna werd het HAVO/MBO diploma gehaalt in 1996 aan het Eemsmondcollege in Delfzijl. In hetzelfde jaar begon hij zijn studie tot electrotechnisch ingenieur aan de Hanzehogeschool te Groningen. De eerste stage werd gelopen bij Philips Semiconductors in Nijmegen op de Quality \& Reliability afdeling. Daarbij werden electromigratiemetingen aan aluminium interconnects en degradatiemetingen aan transistoren uitgevoerd. Daarnaast werd er verder gewerkt aan statistische analyse software voor het verwerken van betrouwbaarheidsgegevens. Een tweede stage werd samen met Albert Martena aan het Universitair Medisch Centrum Groningen uitgevoerd. Er werd gewerkt aan meetsoftware voor het bepalen van motorische vaardigheden van patiënten met de ziekte van Parkinson. De implementatie van fast-fouriertransformatie algoritmes in assembler en de implemenatie van wavelet-transformatie algoritmes behoorden tot de bezigheden. De afstudeeropdracht betrof de ontwikkeling van een radiografisch bestuurbare robotauto gebruikmakende van een prototype Bluetooth chip en werd samen met Albert Martena uitgevoerd aan de universiteit van Karlskrona/Ronneby, Zweden. Hun afstudeerwerk werd beloond in 2000 met de Telecomcity - Sigma Exallon Systems prijs in de provincie Blekinge, Zweden. Enkele maanden werd het werk voortgezet in de realisatie van een prototype Bluetooth extensie microfoon voor een conferentie telefoon van Konftel AB in Umeå, Zweden. De studie werd opgepakt aan de Universiteit Twente, waar hij de M.Sc. titel in de electrotechniek behaalde in 2004. De afstudeeropdracht werd uitgevoerd binnen de Transducers Science \& Technology groep en betrof de ontwikkeling van kunstmatige haarsensoren gebaseerd op krekel haren. Hij werkte binnen dit project als medewerker-onderzoek tot 2006. Daarna begon hij als beoogd promovendus aan het onderzoek beschreven in dit proefschrift. 



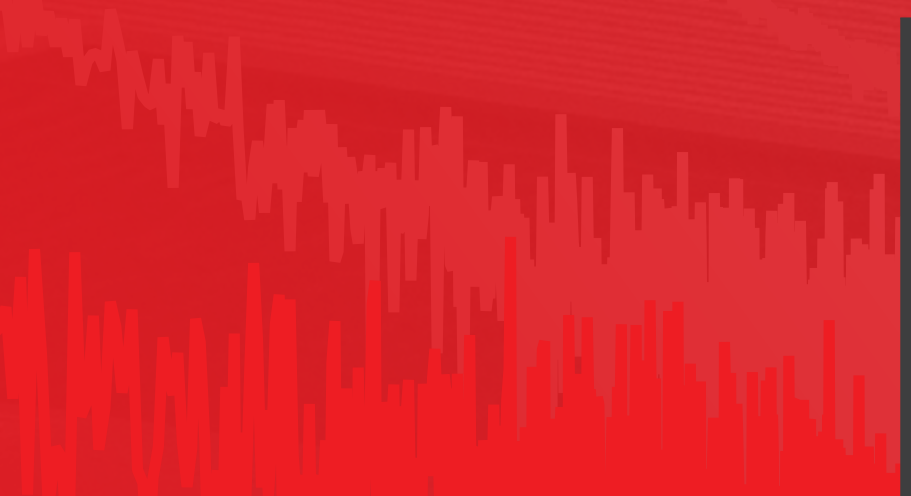

ISBN 978-90-365-2856-6 